\title{
2013 CAEP/ACMU Scientific Abstracts, CAEP 2013 June 1-5, 2013, Vancouver, British Columbia
}

\begin{abstract}
Research is the primary driver of progress and innovation in emergency medicine (EM). It can improve the way we work in the emergency department and impact the care that patients receive across Canada and the world. Where would we be without clinical decision rules, evidence-based practice guidelines, new uses for point-of-care ultrasound, and a better understanding of the causes and potential solutions for overcrowding and access block? Those examples serve only to name a few.
\end{abstract}

The Canadian Association of Emergency Physicians (CAEP) is firmly committed to the advancement of EM research in Canada through its support of established and new researchers.

CAEP administers an annual grant competition, which provides funding for five of the most promising grant proposals submitted. The annual scientific meeting provides an important venue for the dissemination of research activity in Canada and beyond, with 268 abstracts submitted for presentation in 2013. All accepted abstracts receive that designation after being scored by peer review. CAEP presents the Grant Innes Award annually to the top-scoring research abstract. This year, we are also honoring the Top Resident, Top New Investigator, and Top Medical Student projects. Travel support is provided to the top eight research submissions by residents who are members of the CAEP.

Disclaimer: The large number of abstracts submitted and the short time interval between submission and publication do not permit communication with authors, abstract revision, or CJEM editorial review. The following abstracts are presented, unedited, as they were submitted to the CAEP Research Committee. Abstract authors are from the department or division of emergency medicine of their respective universities unless otherwise specified.

Note: Please consult the CAEP 2013 Final Program for the order of Research Abstract presentations.

Dr. Paul Atkinson, Chair, CAEP/AMCU Research Committee

\section{CAEP Research Awards 2013}

First Place, Plenary Presentation; Grant Innes Research Paper and Presentation Award

Simon Berthelot, MD

Identification of Emergency-Sensitive Conditions for the Calculation of an Emergency Care-Specific In-Hospital Standardized Mortality Ratio

Second Place, Plenary Presentation

Bader Alyahya, MD

A Prospective Cohort Study to Differentiate Traumatic Tap from True Subarachnoid Hemorrhage

Third Place, Plenary Presentation

Sheldon Cheskes, MD

The Impact of Perishock Pause on Survival From Out of Hospital Shockable Cardiac Arrest During the Resuscitation Outcomes Consortium (ROC) Primed Trial

Fourth Place, Plenary Presentation; Top Resident Abstract Award Sameer Mal, MD

The Impact of Prehospital Noninvasive Positive Pressure Support Ventilation in Adult Patients with Acute Respiratory Distress: A Systematic Review and Meta-Analysis

Top New Investigator Award

Winner: Ka Wai Cheung, MD

Emergency Department Tobacco Cessation Counseling: Implementation and Evaluation of a Community Based Program - A Pilot Study

Top Medical Student Project Award

Winner: Justin Losier

National Survey of Emergency Physicians for TIA Risk Stratification

Consensus and Appropriate Treatment for a Given Level of Risk
Top Pediatric Abstract Award

Winner: Peter Zed

Medication-Related Emergency Department Visits in Pediatrics: A Prospective Observational Study

CAEP Research Grant Winners

(Submitted for proposed research projects)

Kazia Lenz, MD

Pearlly Ng, MD

Waleed Alqurashi, MD

Nathan Hecht, MD

Steven Lin, MD

CAEP Resident Research Abstract Competition

(Submitted for completed research projects)

Sameer Mal, MD

Carmen Hrymak, MD

Daniel Peterson, MD

Mohammed Aldeeb, MD

Karen Leclerc-Gagne, MD

Farooq Khan, MD

Michael Hickey, MD

\section{Conference Abstracts}

1. Plenary Presentations

2. Oral Presentations (including Lightning Oral Presentations)

3. Moderated Posters (including Electronic Moderated Posters)

4. Posters 


\section{Plenary Presentations}

\section{Winner, Grant Innes Research Paper and Presentation Award} PLO1 Identification of emergency-sensitive conditions for the calculation of an emergency care-specific in-hospital standardized mortality ratio

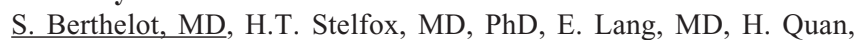
$\mathrm{MD}, \mathrm{PhD}$; Université Laval

Keywords: emergency medicine, emergency-sensitive conditions, health care, International Classification of Diseases, quality indicators Introduction: The Canadian Institute for Health Information (CIHI) provides annual hospital standardized mortality ratios (HSMR) for each Canadian hospital. We developed a HSMR variant to estimate the in-hospital mortality of admitted patients whose emergency department (ED) management would be expected to have an impact (emergencysensitive conditions). Methods: We used a two-step approach to identify emergency-sensitive conditions: (1) Using a modified Delphi approach, a multidisciplinary national panel of emergency care providers and managers $(n=14)$ was presented with the 72 diagnosis groups (DGs) included in the CIHI HSMR. For each DG, the panelists rated on the 9-point RAND/UCLA Appropriateness Method scale to what extent ED management decreases mortality and morbidity and how timely ED care improves patients' prognosis. (2) All members $(N$ $=2,507)$ of the Canadian Association of Emergency Physicians and the National Emergency Nurses Affiliation were presented with the DGs selected as emergency-sensitive conditions for mortality by the modified Delphi approach. Using an electronic survey, they were asked to agree or disagree (binary response) with the panel classification. Results: After panel deliberation, ED care was deemed to potentially reduce mortality and morbidity of, respectively, 37 (e.g., sepsis) and 43 (e.g., atrial fibrillation) of the 72 DGs used for the Canadian HSMR. ED timely interventions were considered to potentially improve the outcome of 40 DGs (e.g., stroke). A list of 47 DGs (e.g., bacterial meningitis) not included in the Canadian HSMR were suggested as additional diagnoses whose mortality could be decreased by appropriate ED care. Of the 37 DGs presented in the national survey, 32 were rated by more than $80 \%$ of respondents $(n=719)$ to be emergencysensitive conditions for mortality. The level of agreement was above $68 \%$ for the five remaining DGs. Conclusion: The development of an emergency-sensitive conditions list will enable the calculation of an inhospital standardized mortality ratio specific to emergency care.

\section{Second Place}

PLO2 A prospective cohort study to differentiate traumatic tap from true subarachnoid hemorrhage

J.J. Perry, MD, MSc, CCFP(EM), B. Alyahya, MD, M. Sivilotti, MSc, MD, FRCPC, M. Bullard, MD, M. Emond, MD, MSc, J. Sutherland, BSc, MEd, A. Worster, MD, C.M. Hohl, MDCM, FRCP, MHSc, J.S. Lee, MD, MSc, M. Eisenhauer, MD, M. Pauls, MD, H. Lesiuk, MD, G.A. Wells, PhD, I.G. Stiell, MD, MSc, FRCPC; University of Ottawa

Keywords: emergency medicine, headache, lumbar puncture, subarachnoid hemorrage

Introduction: Nontraumatic subarachnoid hemorrhage is often a lifethreatening neurosurgical emergency. When a patient presents to the emergency department with sudden severe headache, traditional teaching is to perform a computed tomography scan of the brain and, if negative, a lumbar puncture to analyze the cerebrospinal fluid to exclude subarachnoid hemorrhage. The objective of this study was to assess the cerebrospinal fluid of patients with nontraumatic headache to determine how to distinguish between traumatic tap and subarachnoid hemorrhage. Methods: This was a substudy of a prospective multicentre cohort study. The study was conducted in 12 Canadian academic emergency departments from November 2000 to December 2009. Alert patients over 15 years with an acute nontraumatic headache who underwent lumbar puncture to rule out subarachnoid hemorrhage were included. Results: During our study, there were 4,131 patients enrolled in the Ottawa SAH Rule study; 1,739 patients underwent lumbar puncture and $678(38.9 \%)$ had $>1 \times 10^{6} / \mathrm{L}$ red blood cells in one of the tubes. There were 262 cases of subarachnoid hemorrhage in the entire cohort, 40 of which were diagnosed based on abnormal lumbar puncture results. The presence of less than $9,000 \times 10^{6} / \mathrm{L}$ red blood cells in addition to negative xanthochromia excluded the diagnosis of subarachnoid hemorrhage with a sensitivity of $92.5 \%$ (95\% CI: $80.0-97.0$ ) and a specificity of $97.0 \%$ (95\% CI: $95.0-98.0)$. The sensitivity of visual xanthochromia for subarachnoid hemorrhage was $62.2 \%(95 \%$ CI: 46.1-75.9) and the specificity was $100 \%$ (95\% CI: $99.4-100)$. No cases of subarachnoid hemorrhage, missed by these criteria, were aneurysmal. Two were managed conservatively and one was secondary to a vertebral artery dissection. Conclusion: A combination of negative xanthochromia and red blood cell count $<9,000 \times 10^{6} / \mathrm{L}$ excludes the diagnosis of significant subarachnoid hemorrhage.

\section{Third Place}

PLO3 The impact of perishock pause on survival from out-ofhospital shockable cardiac arrest during the Resuscitation Outcomes Consortium (ROC) primed trial

S. Cheskes, MD, CCFP(EM) FCFP, R. Schmicker, MS, J. Powell, RN, S. May, PhD, J. Menegazzi, PhD, D. Salcido, MPH, R. Verbeek, MD, C. Vaillancourt, MD, MSc, FRCPC, CSPQ, S.C. Brooks, MD, MHSc, R. Berg, MD, A. Idris, MD, R. Sell, MD, M. Kampp, MD, T. Schmidt, MD, P. Owens, MD, J. Christenson, MD; Sunnybrook Centre for Prehospital Medicine

Keywords: cardiopulmonary resuscitation, emergency medicine, heart arrest, resuscitation

Introduction: Previous research has demonstrated significant relationships between perishock pause and survival to discharge from out-ofhospital (OOHCA) shockable cardiac arrest. Limitations to this research include small sample sizes and limited participation by all ROC sites. We sought to determine the impact of perishock pause on clinical outcomes during the ROC PRIMED randomized controlled trial. Methods: We included OOHCA patients in the ROC PRIMED trial who suffered arrest between June 2007 and November 2009, presented with a shockable rhythm, and had CPR process data for at least one shock. We excluded patients who received public access defibrillation before EMS arrival, EMS-witnessed arrest, or those who had missing survival to hospital discharge or Utstein variable data. We used multivariable logistic regression to determine the association between perishock pause duration and survival to hospital discharge. Results: Among 2,046 patients studied (78.3\% male), the median shock pause duration (IQR) was preshock pause 15.0 seconds $(8.0,22.0)$ postshock pause 6.0 seconds $(4.0,9.0)$, and perishock pause 22.0 seconds $(14.0$, 31.0). In an analysis adjusted for Utstein predictors of survival (age, sex, location, bystander-witnessed status, bystander CPR, arrive scene time, and ROC site) as well as CPR quality measures (compression rate, depth, and CPR fraction), the odds of survival to hospital discharge were significantly higher for patients with preshock pause $<10$ seconds (OR: $1.9,95 \%$ CI: $1.35,2.66)$ and perishock pause $<20 \mathrm{sec}-$ onds (OR: $2.07,95 \%$ CI: $1.34,3.21)$ when compared to patients with preshock pause $>20$ seconds and perishock pause $>40$ seconds. Postshock pause was not significantly associated with survival to hospital discharge. Conclusion: In patients with cardiac arrest presenting in a shockable rhythm during the ROC PRIMED trial, shorter pre- and perishock pauses were significantly associated with higher odds of sur- 
vival. Future cardiopulmonary education and technology should focus on minimizing all preshock pauses.

\section{Top Resident Abstract}

PLO4 The impact of prehospital noninvasive positive pressure support ventilation in adult patients with acute respiratory distress: a systematic review and meta-analysis

S. Mal, MD, S.L. McLeod, MSc, A. Iansavichene, BSc, MLIS, A. Dukelow, MD, FRCPC, M. Lewell, MD, FRCPC; The University of Western Ontario

Keywords: emergency medicine, NIPPV, prehospital, severe respiratory distress

Introduction: Noninvasive positive pressure ventilation (NIPPV), which includes continuous and bilevel pressure modalities, has been shown to reduce mortality, intubation rates, and intensive care unit (ICU) length of stay (LOS) for patients admitted to hospital with acute pulmonary edema and acute exacerbation of chronic obstructive pulmonary disease. NIPPV is increasingly being used by emergency medical services (EMS) for the treatment of respiratory distress in the prehospital setting. The primary objective of this systematic review was to determine if prehospital NIPPV for the treatment of adults with severe respiratory distress reduces 30-day mortality compared to "standard" therapy. Secondary objectives were to examine the effect of prehospital-administered NIPPV on the need for invasive ventilation, ICU LOS, and hospital LOS. Methods: Electronic searches of Medline, EMBASE, Cochrane Central Register of Controlled Trials, and CINAHL were conducted and reference lists for relevant articles were hand searched. Randomized controlled trials comparing the use of prehospital NIPPV to standard therapy in adults (age $\geq 16$ years) with severe respiratory distress published in the English language were included. Two reviewers independently screened the titles and abstracts, assessed the quality of the studies, and independently extracted data. Where appropriate, data were pooled using randomeffects models and reported as risk ratios (RR) with $95 \%$ confidence intervals (CIs) and number needed to treat (NNT). Results: Seven randomized controlled trials were included with a combined total of 632 patients; 313 in the standard therapy group and 319 in the NIPPV group. In patients treated with prehospital NIPPV, the pooled estimate showed a reduction in both 30 -day mortality (RR: $0.58 ; 95 \%$ CI: 0.35 , $0.95 ; \mathrm{NNT}=17)$ and need for invasive ventilation (RR: $0.37 ; 95 \% \mathrm{CI}$ : $0.24,0.58$; NNT $=8$ ). There was no difference in ICU or hospital LOS. Conclusion: Out-of-hospital administration of NIPPV appears to be an effective therapy for adult patients with severe respiratory distress. Further research regarding the prehospital administration of NIPPV for severe respiratory distress should aim to delineate its safety and efficacy profile in expanded disease processes such as asthma and pneumonia.

\section{Oral Presentations}

OP01 Medication-related emergency department visits in pediatrics: a prospective observational study

P.J. Zed, PharmD, K.J. Black, MD, MSc, E.A. Fitzpatrick, RN, MN, ENC(C), S. Ackroyd, PhD, N.G. Murphy, MD, J.A. Curran, PhD, N.J. MacKinnon, PhD, D. Sinclair, MD; University of British Columbia

Keywords: adverse drug events, emergency medicine, patient safety, pediatrics

Introduction: There are few data on the rate and characterization of medication-related visits (MRV) to the emergency department (ED) in pediatric patients and none available in Canada. Our objective was to determine incidence, severity, preventability, and classification of
MRVs in the ED of a large tertiary care Canadian pediatric teaching hospital. Methods: A prospective, observational study of pediatric patients presenting to the ED over a 12 -month period was conducted. Patients were randomly selected for inclusion using a systematic sampling methodology stratified a priori by time of day and day of week to ensure a generalizable sample. A pharmacist assessed subjects to determine if their visit was drug related according to one of eight categories. Severity and preventability were classified using predefined definitions. Primary outcomes were reported as proportions presented as percentages with $95 \%$ confidence intervals (CIs). Results: A total of 2,028 patients were enrolled (mean age $6.1 \pm 5.0$ years, $52.6 \%$ female). A MRV was identified in $8.0 \%$ (95\% CI 7.0-9.3), of which $65.0 \%$ (95\% CI 57.2-72.3) were deemed preventable. Severity was classified as mild, moderate, and severe in $8.6 \%$ (95\% CI $4.8-14.0)$, 85.9\% (95\% CI 79.6-90.8), and 5.5\% (95\% CI 2.6-10.2) of cases, respectively. The most common reasons for MRVs were adverse drug reactions $26.4 \%(95 \%$ CI $19.8-33.2)$, subtherapeutic dose $19.0 \%$ (95\% CI 13.3-25.9), and nonadherence $17.2 \%$ (95\% CI 11.7-23.9). A total of 201 drugs were implicated in 163 MRV cases; $136(83.4 \%)$ patients had 1 drug implicated, $16(9.8 \%)$ had 2 drugs implicated, and $11(6.8 \%)$ had 3 drugs implicated. The most common drug classes associated with MRVs were antimicrobial agents (27.4\%), respiratory agents $(22.4 \%)$, central nervous system agents $(20.4 \%)$, immunosuppressants $(7.5 \%)$, and gastrointestinal agents $(6.0 \%)$. Conclusion: A drug-related cause was found in approximately 1 of every 12 pediatrics ED visits, of which two-thirds were deemed preventable. Medication-related visits to the ED are a significant problem that merits further research and intervention.

OP02 Emergency department tobacco cessation counseling: implementation and evaluation of a community-based program: a pilot study

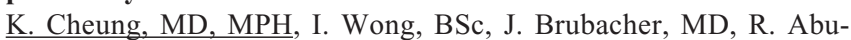
Laban, MD, MHSc, M. Schulzer, MD, PhD; Vancouver General Hospital, Department of Emergency Medicine, University of British Columbia

Keywords: emergency medicine, public health, tobacco use cessation Introduction: Tobacco smoke remains the leading cause of preventable deaths in Canada. Approximately 5\% of emergency department (ED) visits are directly related to tobacco smoke. Currently, emergency physicians are not routinely counseling patients to stop smoking. In this study, we explore whether a brief intervention followed by referral to our provincial telephone quit line would increase quit rates among stable adult smoking patients in the ED. Methods: This was a pilot randomized controlled trial conducted at the Vancouver General Hospital ED from June-August 2011. Stable patients $>19$ years of age presenting to the ED who used tobacco within the last 30 days were eligible. Patients in the control arm received no counseling and patients in the intervention arm were offered a brief intervention and referral to the quit line. Data were collected from each patient on ED arrival and at 1, 3, 6, and 12 months. Results: Fifty-three patients were enrolled. Twenty-six patients were in the control arm and 27 were in the intervention arm. At 12 months, 39\% in the control arm $(n=18)$ made a quit attempt (stopped smoking for 7 days continuously) and $80 \%$ in the intervention arm $(n=5)$ made a quit attempt. At 12 months, $22 \%$ in the control arm had stopped smoking for 30 days continuously and $60 \%$ in the intervention arm had stopped smoking. Conclusion: ED smokers can be identified, provided with brief counseling, and referred to our provincial quit line. Sixty percent of eligible patients accepted referral. Overall, referral of ED smokers may potentially increase quit attempts and quit rates. A full-scale randomized controlled trial is under way to explore these findings. 
OP03 Does regionalization make a difference? An analysis of the differential impact of the Alberta overcapacity initiatives

E. Lang, MD, A. McRae, MD, G. Innes, MD, B.R. Holroyd, MD, MBA, FRCPC, FACEP, M. Bullard, MD, J. Andruchow, MD, MSc, B Li, MA, M. Liu, PhD, B.H. Rowe, MD, MSc, CCFP(EM); University of Calgary

Keywords: emergency crowding, emergency medicine, operations research, overcapacity protocols

Introduction: In December of 2010, Alberta Health Services (AHS) launched a system-wide intervention (SWI) to reduce emergency department (ED) crowding and improve flow. Target hospitals were located in the Calgary and Edmonton Zones, which differ in terms of a regional model of ED functioning as well as inpatient flow redesign strategies. This study compares their SWI implementation on ED and hospital operational metrics. Methods: Using a pre/post and follow-up design, aggregate data were collected on operational metrics for hospital functioning and ED input and throughput. Three 7-month time windows were compared at eight adult Alberta EDs: 01/04-30/10 in each of 2010 (preOCP), 2011 (OCP1), and 2012 (OCP2). Outcomes included ED LOS for all patients, boarding time, and hospital LOS (days) for admitted patients. Parametric bivariable tests were used for all comparisons. Results: This analysis compared three adult hospitals in Calgary to five adult hospitals in Edmonton with 803,000 ED visits over the three time windows. Admissions were higher in Calgary $(23 \%$ v. 17\%), and baseline boarding times were higher in Edmonton (14.6 v. 8.8 hours) hospitals. ED LOS changed from a mean of 7.1 to 5.5 to 5.6 in Calgary, $p<0.001$ for pre to post, while the changes in Edmonton were from 7.4 to 6.1 to $5.5, p<0.001$ for all times. Boarding times in Calgary fell from 8.8 (preOCP) to 3.5 and 3.3 hours in OCP $1+2$; in Edmonton, they fell from 14.6 to 8.1 to 5.1 hours $(p<.001$ for interzone difference). Mean IP LOS changed similarly in both cities; 12.3 to 11.3 to 9.6 in Calgary and 11.6 to 10.9 to 9.4 days ( $p=$ NS for interzone difference). Conclusion: Implementation of a SWI flow initiative was associated with unique improvement profiles related to ED crowding in both zones. While a regionalized model was associated with an accelerated improvement profile, additional reasons for variations in degree of improvement should be analyzed in further detail.

OP04 What is the optimal chest compression depth during out-ofhospital cardiac arrest resuscitation of adult patients?

I.G. Stiell, MD, MSc, FRCPC, S. Brown, PhD, C.W. Callaway, MD, PhD, G. Nichol, MD, T.P. Aufderheide, MD, S. Cheskes, MD, C. Vaillancourt, MD, MSc, FRCPC, CSPQ, D. Hostler, PhD, D.P. Davis, MD, A. Idris, MD, J. Christenson, MD, L. Morrison, MSc, MD, FRCPC, J. Stouffer, EMT-P, C. Free, EMT-P; University of Ottawa, Department of Emergency Medicine

Keywords: cardiac arrest, CPR, emergency medicine, EMS

Introduction: The $2010 \mathrm{AHA} / \mathrm{ILCOR}$ recommendations suggested an increase in CPR compression depth for adults, with a target $>50 \mathrm{~mm}$ and no upper limit. This target is based upon limited evidence; hence, we sought to determine the optimal compression depth range for adult patients. Methods: We studied emergency medical services-treated OOHCA patients from the Resuscitation Outcomes Consortium ROC PRIMED clinical trial and Epistry - Cardiac Arrest database for whom electronic CPR compression depth data were available, from June 2007 to December 2010. We calculated anterior chest wall depression in millimetres for each minute of CPR. We controlled for 10 covariates, including compression rate and calculated adjusted odds ratios for survival to hospital discharge, 24-hour survival, and any return of circulation (ROSC). Smoothing splines were used to explore the relationship between average compression depth and outcome. Results: We included 9,142 adult patients from nine US and Canadian cities with these characteristics: mean age 67.5 years; male $64 \%$; bystander witnessed 44\%; bystander CPR 42\%; initial rhythms - VF/VT 24\%, PEA $20 \%$, asystole $49 \%$, other nonshockable $6 \%$; outcomes - ROSC $31.3 \%$, 1 -day survival $22.8 \%$, survival to hospital discharge $7.3 \%$. For all patients, the mean compression rate was 108 per minute; mean compression fraction 0.68 ; mean compression depth $41.9 \mathrm{~mm}$ with ranges:

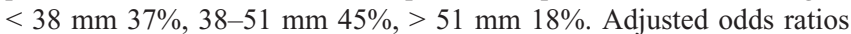
for survival to discharge, with depth $>51 \mathrm{~mm}$ as reference, were < $38 \mathrm{~mm}-0.69$ (95\% CI $0.53,0.90)$ and $39-51 \mathrm{~mm}-1.03(0.81,1.30)$. Results were similar for the intermediate outcomes of ROSC and 1-day survival. Covariate-adjusted spline curves revealed that the maximum survival was associated with a depth of $45.8 \mathrm{~mm}$ followed by a decline in survival by $50 \mathrm{~mm}$ (optimal interval $44-49 \mathrm{~mm}$ ). We also found no differences in the spline curves between males and females. Conclusion: This study found that more than one-third of patients received very low compression depth. The optimal CPR compression depth for survival appears to be $46 \mathrm{~mm}$ (44-49) for both males and female adults but falls off after $50 \mathrm{~mm}$. These findings conflict with the 2010 international guideline recommendations.

\section{OP05 Adverse outcomes in ED presyncope patients: a prospective cohort study}

V. Thiruganasambandamoorthy, CCFP(EM), MSc, A. Vaidyanathan, MBBS, L.A. Calder, MD, MSc, FRCPC, V. Donici, BSc, G.A. Wells, PhD, I.G. Stiell, MD, MSc, FRCPC, University of Ottawa, Department of Emergency Medicine

Keywords: emergency medicine, management, outcomes, presyncope Introduction: Presyncope is the sudden onset of impending loss of consciousness without losing consciousness (which differentiates it from syncope). The goal of this study is to describe the frequency of ED visits, management, and occurrence of adverse outcomes for ED presyncope patients versus syncope patients. Methods: We conducted a prospective study of adult presyncope patients at two academic EDs over 16 months. We included adults with presyncope and excluded patients with mental status changes, seizure, alcohol/illicit drug abuse, and head and significant trauma. We collected patient characteristics, ED management, and short term (30-day) and long term (31 days to 1year) adverse outcomes. The list of adverse outcomes includes MI, pulmonary embolism, subarachnoid and significant hemorrhage, and procedural interventions for short term and death, arrhythmia, and structural heart disease for both short and long terms. We also collected the treating physicians' prediction probability for short-term outcomes. Adverse outcomes were assessed by medical records review and telephone follow-up and confirmed by two blinded ED physicians. Analyses included descriptive and receiver operating characteristic (ROC) curve creation. Results: Of the total 199,975 ED visits during the study period, $976(0.5 \%)$ were for presyncope. We enrolled 713 patients $(73.1 \%)$ with these characteristics: mean age 55.5 years, females $55.4 \%$, and history of syncope $4.4 \%$. ED investigations included blood tests $82.2 \%$, ECG $91.4 \%$, and CT head $13.6 \%$. A minority $(10.0 \%)$ had specialist consultation in the ED, and $4.5 \%$ were admitted (syncope admission rate $8.1 \%$ ). A small proportion $(3.9 \%$ ) suffered minor injuries and $0.3 \%$ suffered fractures. We detected 54 (7.6\%) patients who suffered adverse outcomes: $33(4.6 \%)$ in the short term (death $0.3 \%$, cardiovascular events $2.9 \%$, noncardiac events $1.4 \%)$. Nearly one-half $(2.1 \%)$ of the short-term adverse outcomes occurred after ED disposition. In the long term, an additional 21 $(2.9 \%)$ patients suffered adverse outcomes: $0.8 \%$ death due to unknown/related cause and $1.3 \%$ arrhythmia. The ROC curve for physician judgment for 30-day outcomes was 0.62 (95\% CI 0.49 , 0.75). Conclusion: Presyncope is a common ED problem with similar 
outcome types as syncope. While admission rates are lower than syncope, a significant proportion of patients suffer outcomes after ED discharge and in the long term. There is a need for accurate risk stratification of ED presyncope patients.

\section{OP06 Ultra-low-dose CT comparable to standard CT in patients with suspected renal colic}

J. Archambault, MD, C. Hrymak, MD, S. Sivalingam, MD, I. Kirkpatrick, $\mathrm{BSc}(\mathrm{Gen}), \mathrm{BSc}(\mathrm{Med}), \mathrm{MD}$; University of Manitoba

Keywords: emergency medicine, genitourinary, imaging Introduction: Noncontrast computed tomography (CT) is currently the standard radiologic investigation for patients with suspected renal colic. Especially in patients with multiple scans, concerns about radiation exposure have led to the evaluation of low-dose radiation CT scans in diagnosing renal calculi. Our objective was to compare an ultra-low-dose (study) CT scan protocol to standard CT in the evaluation of patients with suspected renal colic. Methods: Fifty-six emergency room patients with suspected renal colic underwent both a standard and study CT to assess for renal colic. The study CT protocol lowered the radiation exposure approximately $95 \%$ by fixing the tube current at 20 milliampere-seconds (mAs). Both CT scans were read twice by a group of four blinded radiologists. The primary outcome was stone detection rate. Secondary outcomes included signs of obstruction, interobserver agreement, and stone characteristics. Patient body mass index (BMI) and alternative diagnosis were also evaluated. Patients were excluded if they were clinically unstable or unable to provide informed consent. Study approval was received from the institutional health research ethics board. Results: Fifty-six patients had data available for comparison. Thirty-three of the 36 patients with stones on standard CT were identified on the study CT. The sensitivity and specificity for stone detection were $92 \%$ and $100 \%$, respectively. Sensitivity increased to $>97 \%$ for stones $>2 \mathrm{~mm}$ in size. There was no statistically significant difference in stone detection (McNemar test $p=$ 0.63 , alpha $<0.05)$. Interobserver agreement for stone detection was excellent (all kappa values $>0.8$, combined mean 0.9 ). The only false negative was a $2 \mathrm{~mm}$ distal stone in a patient with a BMI of 48 . Conclusion: Our ultra-low-dose CT protocol is comparable to standard noncontrast CT in patients with suspected renal colic. This strengthens the argument for developing reduced radiation CT protocols for screening patients with suspected renal colic.

OP07 Ten-minute educational intervention improves emergency physicians' ability to interpret left ventricular function

D. Peterson, MD, PhD, S.L. McLeod, MSc, J. Ahn, MD, L. Rang, MD, FRCPC, RDMS, M.Y. Woo, MD, J.S. Chenkin, MD, A. McRae, MD, N. Fine, MD, FRCPC, R. Arntfield, MD, FRCPC, RDMS; The University of Western Ontario

Keywords: emergency medicine, left ventricular function, point of care, ultrasound

Introduction: Global assessment of left ventricular (LV) function is not routinely taught as part of point-of-care ultrasound (US) training for emergency physicians (EPs) in Canada. With emerging interest in this and other "advanced" US applications, developing effective and widely accessible educational strategies is essential. The objective of this study was to evaluate the effectiveness of a brief, online educational tutorial on an EP's accuracy when interpreting LV function. Methods: Academic EPs (residents and staff) who had completed an introductory-level point-of-care US course from five tertiary care centres across Canada (Ottawa, Kingston, Toronto, London, Calgary) were invited to participate. Using two basic echocardiographic views of the heart, a selection of point-of-care US video clips obtained by trained EPs on real patients with varying degrees of LV function were sent to an echocardiologist for expert grouping into four broad categories. Following a pretest of selected video clips, participants viewed a 10-minute online tutorial explaining how to interpret LV function using point-of-care US. Acting as their own control, participants then completed a posttest composed of different video clips. Pre- and posttest scores were compared using a paired-samples $t$-test, and general linear models with repeated measures were used where appropriate. Results: The response rate was $48.5 \%$ (160/330). The majority of respondents $(72.5 \%)$ were between the ages of 25 and 44 years, and $117(73.1 \%)$ were male. Sixty-three (39.4\%) participants reported they had completed an "advanced" application US course. Ninety-six $(60.0 \%)$ participants reported they were "not at all comfortable" interpreting LV function images with point-of-care US. There was a significant increase from the pretest $(53.2 \%)$ to posttest $(70.7 \%)$ in correct interpretations of LV function $(\Delta 17.5 \% ; 95 \% \mathrm{CI}: 15.3,19.7)$. There was no difference between physicians who had taken a basic versus an "advanced" application US course, institution, age group (18-24, 25$34,35-44,45-54,55+$ years), or years of emergency medicine practice $(0-4,5-9,10-14,15+$ years). Conclusion: This brief, online educational intervention significantly improves the accuracy of EP interpretation of global LV function. This is an effective, easily accessible online tutorial that could be used as part of a comprehensive educational program for point-of-care US.

OP08 Network meta-analysis: prioritizing corticosteroid treatments to prevent relapse after acute asthma discharge

B.H. Rowe, MD, MSc, CCFP(EM), S. Kirkland, BSc, MSc, E. Cross, MD (Candidate), T.Y. AlShawabkeh, MD, JBEM, B. Vandermeer, MSc, S. Campbell, BA, MLs, C. Villa-Roel, MD, PhD (Candidate); University of Alberta

Keywords: asthma, corticosteroids, emergency medicine, relapse Introduction: Systemic corticosteroids are the treatment of choice to reduce relapse after discharge from the emergency department for acute asthma; however, the most effective route of administration is unclear. The objective of this study was to compare the efficacy of intramuscular (IM) or oral corticosteroids in reducing relapses after ED discharge. Methods: A search of eight electronic databases including Medline, EMBASE, CINAHL, and Cochrane was completed. Grey literature searching (hand searching, Google, and SCOPUS) was also conducted. Finally, the Cochrane Airways Group (CAG) provided search results for the period 2002-2012. Studies were included if they were controlled clinical trials comparing the effectiveness of either intramuscular (IM) or oral corticosteroids to reduce relapse after ED (or equivalent setting) discharge for acute asthma in children or adults. Two independent reviewers judged the relevance, inclusion, and risk of bias (RoB) of the studies. Individual and pooled statistics were calculated as odds ratios (OR) with $95 \%$ confidence intervals (CI). Results: From 2,708 citations, 26 studies were included in this review. Of these, 5 compared either IM or oral corticosteroids to placebo, 10 compared IM to oral corticosteroids, and 11 compared differing oral corticosteroid regimens. Fourteen were pediatric and 12 were adult; the overall RoB of studies was unclear. In the 10-day relapse analysis, IM (OR 0.15; 95\% CI: $0.5,0.38$ ), short-course oral (OR 0.16; 95\% CI: 0.4 , $0.50)$, dexamethasone $(\mathrm{OR}=0.21 ; 95 \% \mathrm{CI}: 0.05,0.81)$, and longcourse oral (OR $0.23 ; 95 \%$ CI: $0.09,0.55)$ corticosteroids are superior to placebo. IM (51\%) and short-course oral $(31 \%)$ were the treatments most likely to be the best treatment. At 21 days, the odds ratios were closer to the null; dexamethasone $(\mathrm{OR}=0.34 ; 95 \% \mathrm{CI}$ : $0.02,11.5)$, long-course oral (OR 0.36; 95\% CI: 0.07, 2.26), IM (OR 0.36; 95\% CI: $0.5,3.5$ ), short-course oral (OR $0.45 ; 95 \%$ CI: $0.04,7.8$ ), and not statistically significant, although this could be due to much smaller sample sizes. These smaller sample sizes also precluded more detailed subgrouping. Conclusion: Overall, corticosteroids reduced early relapse 
after discharge, with preference for IM or short-course delivery. These results confirm guideline recommendations; however, consideration of IM agents in discharged patients appears warranted. Patient acceptance of this strategy was not assessed.

OP09 Evaluation of Ontario's Emergency Department Process Improvement Program on emergency department waiting times M. Schull, MD, MSc, R. Bell, MD, MSc, T. Stukel, PhD, M. Vermeulen, MSc, M. Zwarenstein, MBBCh, MSc, B.H. Rowe, MD, MSc, CCFP(EM), A. Guttmann, MDCM, MSc, G. Anderson, MD, PhD, A Sales, RN, PhD, A. Nigam, PhD, D. Carew, MN, B. Golden, PhD, T. Rutledge, MD; Sunnybrook and Women's Hospital

Keywords: ED wait times, emergency medicine, process improvement Introduction: In 2008, Ontario launched the Emergency Department (ED) Wait Times Strategy to reduce overcrowding. One intervention, the ED Process Improvement Program (ED-PIP), was a structured 8month program based on Lean management principles, implemented fully in 54 hospitals in four waves from 2009 to 2011. We sought to evaluate the effect of PIP on ED length of stay (LOS) in the first three waves. Methods: ED-PIP and frequency-matched comparator hospitals were evaluated using health administrative data. ED-PIP and comparator sites in each wave were analyzed separately. Baseline linear trends ( 2 years prior to ED-PIP) were compared to outcomes 6 months after the end of ED-PIP using linear GEE models. Important patient and contextual factors were controlled for. The primary outcomes were ED-LOS and time to physician initial assessment (PIA). Other quality of care outcomes were analyzed as potential unintended consequences. Results: We analysed five wave 1 ED-PIP hospitals (with 43 comparator sites); waves 2 and 3 had 16 and 15 ED-PIP sites (with 18 and 8 comparator sites), respectively. In adjusted models, ED-PIP wave 1 was not associated with improved ED LOS or PIA. Wave 2 and 3 EDPIP was independently associated with small but significant reductions in median ED LOS (aOR -0.15 [95\% CI $-0.21,-0.09$ ]; aOR -0.13 $[95 \% \mathrm{CI}-0.21,-0.06]$, respectively) and 90th percentile PIA (aOR 0.11 [ $95 \%$ CI $-0.17,-0.05]$; aOR -0.29 [95\% CI $-0.35,-0.23]$, respectively). ED-PIP was associated with reduced LWBS in all three waves; no other significant differences in quality of care outcomes were observed. Conclusion: In controlled analyses, wave 2 and 3 ED-PIP was independently associated with small but significant reductions in some ED-LOS and PIA measures. LWBS rates were reduced in all three waves, and no adverse effects were seen.

$O P 10$ A delphi process to identify interventions to improve bystander CPR rates among Canadian seniors

C. Vaillancourt, MD, MSc, FRCPC, CSPQ, A. Kasaboski, BSc, M. Charette, MSc, J. Grimshaw, MBChB, PhD, J. Brehaut, PhD, M Osmond, MD CM, I.G. Stiell, MD, MSc, FRCPC, G.A. Wells, PhD; Ottawa Hospital Research Institute

\section{Keywords: bystander, CPR}

Introduction: Bystander CPR rates remain low among people aged $\geq$ 55 , the group most likely to witness out-of-hospital cardiac arrest (OHCA). We recently completed a national survey that provided insight into the factors influencing acquisition of CPR knowledge and skills in that group. We sought to identify stakeholders most likely to gain from our national CPR survey findings and engage them in an intervention planning exercise to improve bystander CPR rates among seniors. Methods: We used a modified Delphi method to identify consensus-based recommendations on how to improve bystander CPR rates. We used a snowball sampling strategy to identify Canadian stakeholders within six spheres: seniors' groups, medical experts, researchers, CPR educators, government officials, and the media. First, we presented the national survey-derived factors affecting seniors' intention to learn or perform CPR to participating stakeholders and asked them to recommend suitable intervention and policy functions in round table discussions. We later asked participants to score all recommendations for priority, feasibility, and potential impact on a 5-point Likert scale. Recommendations reached consensus if $75 \%$ of participants scored them $\geq 4 / 5$ (descriptive statistics). Results: Among 195 identified stakeholders, 43 from all six spheres attended the round table discussions, and 5 more participated in the Delphi exercise. The first round (response rate $83.3 \%$ ) included 198 recommendations, 109 of which reached consensus. We discarded 7 among the remaining 89 for lack of clarity, and participants added 4 new recommendations. Among these 86 recommendations, an additional 36 reached consensus in the second Delphi round (response rate $70.0 \%$ ). The 145 recommendations featured these intervention functions: enablement 40 (27.6\%), education $27(18.6 \%)$, persuasion $22(15.2 \%)$, environmental restructuring $21(14.5 \%)$, training $20(13.8 \%)$, modeling $9(6.2 \%)$, and incentivization $6(4.1 \%)$. The 10 top-ranked recommendations suggest the best intervention should (1) highlight CPR benefits and dispel misconceptions using simple messaging; (2) encourage action; (3) facilitate skill acquisition via outreach programs; and (4) saturate the media in a sustained manner until becoming part of the community fabric. Conclusion: We successfully identified and engaged stakeholders in a consensus-based exercise resulting in multifaceted recommendations most likely to increase bystander CPR and survival rates for OHCA.

OP 11 Safety of a system-wide intervention to address access block in 13 congested Alberta hospital EDs

A. McRae, MD, E. Lang, MD, B. Li, MA, M. Liu, PhD, D. Wang, PhD, M. Bullard, MD, B.R. Holroyd, MD, MBA, FRCPC, FACEP, B.H. Rowe, MD, MSc, CCFP(EM), G. Innes, MD; University of Calgary

Keywords: access block, crowding, emergency medicine, overcapacity protocols

Introduction: A system-wide intervention (SWI) to reduce ED crowding, which included an overcapacity protocol (OCP), was implemented across Alberta in December of 2010. The SWI focused on improving flow and transferring admitted patients to nontraditional inpatient care spaces when the ED load reached predefined thresholds. The objective of this project was to examine the safety of this strategy to alleviated ED access block. Methods: A pre-post follow-up design study was conducted examining the impact of the Alberta SWI on patient safety. Three time periods were compared at 13 urban adult Alberta EDs: 01/04-30/10 in each of 2010 (preSWI), 2011 (SWI1), and 2012 (SWI2). Outcomes of interest included unscheduled ED visits and admissions within 3 and 7 days, both for patients discharged from the ED and for patients discharged from hospital. Parametric bivariable tests and regression models were used for all comparisons. Results: This analysis included 1.5 million ED visits and over 210,000 hospitalizations for the three time periods with a 3 to $6 \%$ annual increase in $\mathrm{ED}$ volume. Among patients discharged from the ED, $7.1 \%$ of patients had an unplanned ED visit within 3 days of discharge in the preSWI period compared to $6.9 \%$ in SWI1 and $6.6 \%$ in SWI2 $(p<0.001)$. Of discharged ED patients, $11.3 \%$ had an unplanned ED visit within 7 days preSWI, compared to $10.9 \%$ in SWI1 and $10.7 \%$ in SWI2 $(p<0.001)$. More patients discharged from inpatient units had unplanned ED visits within 3 and 7 days of discharge following SWI implementation (3-day ED visits $3.6 \%$ preSWI, 3.7\% SWI1, 3.9\% SWI2, $p=0.04$; 7-day ED visits $8.4 \%$ preSWI, $8.6 \%$ SWI1, $8.9 \%$ SWI2; $p=0.003$ ). There was no statistically significant difference between the three study periods in ED visits requiring readmission for patients discharged from inpatient units. Conclusion: SWI implementation in 13 hospitals does not appear to result in increased unscheduled ED return visits. A minor 
increase in ED visits among patients discharged from inpatient units was seen following SWI implementation; however, no significant increase in short-term readmissions was observed. This intervention has effectively improved ED and hospital access block without compromising ED patient safety; efforts to reduce return ED visits after inpatient discharge would further improve ED patient flow.

OP12 Improved thrombolysis rates and quality of care for stroke patients through a provincial emergency department quality improvement initiative

D.R. Harris, MD, MHSc, PhD(c), R. Stenstrom, MD, PhD, E. Grafstein, MD, G. Innes, MD, P. Lindsay, RN, PhD, J. Singer, PhD, M. Collison, MHA; Department of Emergency Medicine, St. Paul's Hospital

Keywords: cerebrovascular diseases, emergency medicine, performance measurement, quality improvement

Introduction: The care of stroke patients in the emergency department (ED) is time sensitive and complex. We sought to improve quality of care for stroke patients in British Columbia (BC) emergency departments. The objective was to implement and measure the outcomes of a large-scale quality improvement initiative on thrombolysis rates and other ED stroke performance measures. Methods: This was an evaluation of a large-scale quality improvement initiative, within EDs in $\mathrm{BC}$, in a before-after design. Baseline data were derived from a medical records review study performed between December 1, 2005, and January 31,2007 . Adherence to best practice was determined by measuring selected performance indicators. The quality improvement initiative was a collaboration between multidisciplinary clinical leaders within EDs throughout BC in 2007, with a focus on implementing clinical practice guidelines and preprinted order sets. The post data were derived through an identical methodology as baseline, from March to December 2008. The primary outcome was the thrombolysis rate; secondary outcomes consisted of other ED stroke performance measures. Results: Forty-eight of $81(59 \%)$ acute care hospitals in BC were randomly selected for audit in the baseline data, with selected sampling by Health Authority and hospital size (comprehensive stroke centres were all included). A total of 1,258 TIA and stroke charts were audited at baseline. For post data, 46/81 (57\%) acute care hospitals were sampled, representing the identical set at baseline; 1,199 charts were audited. The primary outcome of thrombolysis rate was 3.9\% (23/564) before and 9.3\% (63/676) after, an absolute difference of 5.4\% (95\% CI: $2.3-7.6 ; p$ $=0005)$. Other measures showed changes: administration of aspirin to stroke patients in the ED improved from $23.7 \%$ (127/535) to $77.1 \%$ $(553 / 717)$, difference $=53.4 \%(95 \%$ CI: $48.3-58.1 \% ; p=0005)$; and door to imaging time improved from 2.25 hours (IQR $=3.81$ hours) to 1.57 hours (IQR 3.0 hours), difference $=0.68$ hours $(p=0.03)$. Differences were found in improvements between large and small institutions and between health regions. Conclusion: Implementation of a provincial emergency department quality improvement initiative showed significant improvements in thrombolysis rates and adherence to other best practices for stroke patients. The specific factors that influence improvement need to be further explored.

$O P 13$ Evaluation of a secure, store-and-forward teledermatology system to facilitate emergency physician consultations

M. Haager, MD, A. Mirza, BSc, MD, CCFP(EM), J. Rao, MD, FRCPC; University of Alberta

Keywords: dermatology, emergency medicine, teleconsult Introduction: We describe an ongoing store-and-forward teledermatology (SFT) pilot project in Edmonton, Alberta. The objectives of this study were to (1) determine emergency physician (EP) satisfaction with SFT; (2) document patient satisfaction with the project; and (3) evaluate the efficiency and practicality of remote dermatology consul- tation. Methods: Patients with skin-related complaints were invited to participate in a pilot teledermatology project involving two communitybased emergency departments (EDs) and a university-based dermatology centre. Patients were selected at the discretion of the attending EP. Digital photographs of the patient's skin lesions and the ED chart were obtained. All images were uploaded to a secure and confidential Webbased teledermatology platform that met the required privacy standards. After remote review, the teledermatologist provided (1) a diagnostic impression, (2) an educational note, and (3) management suggestions. All patients were invited for follow-up. All consults were obtained in real time 24 hours a day, 7 days a week. Participating emergency physicians, as well as all patients who attended dermatology follow-up, were invited to complete a survey documenting their satisfaction and experience with the process. Results: A total of 480 patients (233 males and 247 females) participated in the first 12 months of this project, ranging from 0 to 97 years of age. Average time from consult request to completion was 16 minutes (range 1 to 47 minutes). As determined by the teledermatologist, the top three presenting diagnoses were (1) dermatitis and variants, (2) drug eruptions of various types, and (3) vasculitis. Ninety-six percent of patients received prescription recommendations, $22 \%$ received surgical recommendations, and 5\% required only reassurance. All patients had the option of dermatology follow-up. No patient required on-site dermatologist visitation, and none were recommended to be referred to another specialist or service. Sixty-seven percent of emergency physicians responded to the survey, and all reported overall satisfaction with the process. Of the 480 participating patients, 52 were subsequently seen in clinic for dermatology follow-up. Ninety percent completed surveys at the time of follow-up. and all reported overall satisfaction with the teledermatology process. Most of the patients seen in follow-up had resolution of their skin conditions as a result of the teledermatology management suggestions. Conclusion: SF teledermatology can improve patient care, expedite the consultation process, offer timely access to specialist care, and contribute to continuing medical education. It may also represent a cost-saving opportunity by avoiding unnecessary repeat visits.

OP14 The impact of massed versus spaced instruction on learning of procedural skills in pediatric resuscitation F.A. Khan, MDCM, C. Patocka, MDCM, F. Bhanji, MD, MSc, I. Bank, MDCM, FRCPC, FAAP, A. Dubrovsky, MDCM, MSc, FRCPC, D. Brody, MD, FRCPC; McGill Emergency Medicine Residency Program

Keywords: education, emergency medicine, procedures, teaching Introduction: Survival from cardiac arrest has been linked to the quality of resuscitation care. Unfortunately, health care providers frequently underperform in these critical scenarios, with a well-documented deterioration in skills following an advanced life support course. Improving initial training and preventing decay in knowledge and skills are a priority in resuscitation education. The purpose of this study was to determine if a resuscitation course taught in a spaced format compared to the usual "massed" instruction results in improved procedural skills. Methods: We delivered a case-based pediatric resuscitation course to two cohorts of medical students: one in a spaced format (four 75-minute weekly sessions) and the other in a massed format (a single 5-hour session). Four weeks following course completion, blinded observers assessed each learner at various skills stations. Primary outcomes were performance on bag-valve-mask ventilation (BVMV), intraosseous (IO) insertion, and chest compressions using expert-developed checklists. Secondary outcomes included performance of "key components" of the above skills. Results: Forty-five of 48 students completed the study protocol (23 spaced and 22 massed). Students in the spaced cohort scored higher overall for BVMV $(6.9 \pm 1.4$ v. $5.8 \pm 1.9, p<0.04)$, without significant differences in scores for IO insertion $(3.9 \pm 1$ v. $3.7 \pm 1.2, p=0.575)$ and chest compressions $(10.9 \pm 2.7$ v. $10.1 \pm 2.4, p=0.342)$. They were also 
more likely to administer oxygen during BVMV (OR 47.2, 95\% CI 5.2$423, p<0.001$ ), adhere to a target ventilation rate (OR 4.9, 95\% CI $1.1-$ $21.2, p<0.03$ ), use a stool when appropriate for chest compressions (OR $8.3,95 \%$ CI $1.2-59, p<0.03)$, and landmark correctly for IO insertion (OR 5.4, 95\% CI 1.3-24.3, $p<0.02$ ). The intervention group also had a significantly shorter mean time to IO insertion $(30.2 \pm 34$ seconds $\mathrm{v}$. $62.1 \pm 30$ seconds, $p=0.002$ ). Conclusion: Infrequent yet critically important procedures learned in a spaced format may result in better skill retention and more efficient task completion when compared to traditional massed training.

OP15 Bedside lung ultrasound for the diagnosis of pulmonary edema in patients presenting with acute dyspnea: a systematic review and meta-analysis

M.S. Aldeeb, MBBS, D. Barbic, MD, S. Barbic, MSc, R. Featherstone, MLIS, J. Dankoff, MD; McGill University

\section{Keywords: emergency medicine, ultrasound}

Introduction: Acute dyspnea is a common presenting complaint to the emergency department. The rapid diagnosis of pulmonary edema is critical for appropriate treatment, yet establishing the wrong diagnosis can have detrimental effects for the patient. Recent studies suggest that the use of lung ultrasound may aid in the diagnosis of pulmonary edema in patients presenting with acute dyspnea. Methods: A systematic review protocol was created to guide the search and analysis, and we searched the following databases: PubMed, EMBASE, Ovid MEDLINE, Ovid MEDLINE In-Process \& Other Non-Indexed Citations, and the Cochrane Database of Systematic Reviews. References of reviewed articles were hand-searched, as were the conference abstracts from major emergency medicine and critical care conferences. We included prospective, original studies that recruited patients presenting to hospital with symptomatic, acute dyspnea or where there was a clinical suspicion of congestive heart failure. Two reviewers independently reviewed all citations to assess for inclusion, abstracted data, and assessed included studies for methodological quality. Contingency tables were used to calculate sensitivity, specificity, and likelihood ratios. Results: The sensitivity (SEN) of bedside ultrasound for pulmonary edema is $94.1 \%$ (95\% CI $81.3-98.3$ ). The specificity (SPEC) of ultrasound for pulmonary edema is $92.3 \%$ (84.2-96.4). The positive likelihood ratio is 12.4 (5.7-26.8), and the negative likelihood ratio is 0.06 (0.02-0.22). Conclusion: Bedside lung ultrasound has excellent values for sensitivity and specificity when compared to the gold standard, final discharge diagnosis of heart failure by independent cardiologists blinded to the ultrasound results. This is a promising tool for the evaluation of the acutely dyspneic patient.

OP16 Disposition of emergency department patients with acute pulmonary embolism in a Canadian health region

G. Innes, MD, D. Wang, PhD, E. Lang, MD, J. Andruchow, MD, MSc, A. McRae, MD; University of Calgary

Keywords: emergency, emergency medicine, pulmonary embolism Introduction: Emergency department (ED) management and disposition of patients with acute pulmonary embolism (APE) are variable. There is evidence that outpatient treatment is safe in some cases. Our objective was to describe the incidence and disposition of ED patients with APE in a Canadian health region. Methods: This administrative database study included all Calgary ED patients who received a diagnosis of APE in 2012. The number of nuclear (VQ) and CT PE studies was obtained from the regional DI database, and discharge diagnoses were based on ED and hospital ICD-10 discharge records. Prior ED visits and 30-day hospitalization rates were collated from the regional ED database. Results: During the study, 243,858 patients presented to the three hospitals; 3,212 underwent PE imaging (2,935 CT, $240 \mathrm{VQ})$ and 505 (15.7\%) had radiographically confirmed APE. Mean age was 58.0 years (SD 17.8), 51.7\% were male, $40 \%$ arrived by EMS, and mean triage level was 2.4 (SD, 0.5). Overall, 293 patients (58\%) were admitted and 212 discharged after a median LOS of 9.0 hours. Factors associated with admission included age (mean, 62.1 v. 52.4 years, $p<0.001$ ), male sex $(51.9 \%$ v. $42 \%, p=0.03)$, and EMS arrival $(49.8 \%$ v. $26.4 \%, p, 0.001)$ in the (admitted v. discharged groups), respectively. Fourteen discharged patients $(2.8 \%)$ returned requiring hospitalization within 30 days. Thirty patients $(5.9 \%)$ had a prior ED visit within a week of their PE diagnosis and received non-PE diagnoses of leg pain or DVT (8), back or musculoskeletal pain (3), pneumonia (2), CP NYD (2), dizziness, syncope, abdominal pain, asthma, urticaria, hiccups, failure to thrive, hyperglycemia and other (7). Conclusion: In this setting, $42 \%$ of APE patients were discharged from the ED. The 30-day adverse event rate for discharged patients was low. A small but clinically important number of patients may have had their diagnosis missed during ED visits within 7 days.

OP17 Emergency department-initiated interventions for mild traumatic brain injury: a systematic review

J. Gravel, MD, MSc, A. D'Angelo, MD, B. Carrière, MD, MSc, L. Crevier, MD, M. Beauchamp, PhD, J. Chauny, MD, MSc, M. Wassef, $\mathrm{MSc}, \mathrm{N}$. Chaillet, PhD; Université de Montréal

Keywords: emergency department, emergency medicine, intrevention, mild traumatic brain injury

Introduction: Most patients suffering from mild traumatic brain injury (mTBI) present persistent symptoms at 1 week and 1 month following injury. The primary objective of this systematic review was to investigate the effectiveness of interventions initiated in the emergency department (ED) for adults and children who have sustained acute mTBI. Methods: We performed a systematic review of all randomized clinical trials evaluating any intervention initiated in the ED or in an acute setting for patients suffering an acute mTBI. All possible outcomes were included. The primary source of identification was Medline, Embase, PsycINFO, CINAHL, and Cochrane Central Register of Controlled Trials from 1980 until August 2012. Hand search of proceedings from five different meetings related to mTBI was performed. Study selection was conducted by two coauthors while data abstraction was completed by a research assistant specialized in systematic review. Study quality was evaluated using Cochrane's risk of bias assessment tool. Results: From a potential of 15,156 studies, 1,268 abstracts were evaluated and 120 articles were completely read. Among these, 15 studies fulfilled the inclusion/exclusion criteria. One study evaluated a pharmacologic intervention (DDAVP), two evaluated activity restriction, one evaluated head CT scan versus admission, four evaluated an information intervention (pamphlet, information session at the ED), and seven evaluated different follow-up interventions. These studies used different outcome measures and measurement intervals, thus limiting the possibility for analysis. However, the meta-analysis of three studies ( $n=620$ patients) evaluating a different follow-up strategies versus routine or no follow-up failed to show an impact on memory (RR 1.17; 95\% CI: 0.74-1.86), headache (RR 0.98; 95\% CI: 0.82-1.17), and irritability (RR: 1.03 ; 95\% CI: $0.79-1.35$ ) at 6 to 12 months posttrauma. Also, the meta-analysis of two studies ( $n=269$ patients) showed no impact of an information intervention on headache at 3 months (RR: 0.88 ; 95\% CI: $0.65-1.19$ ). Conclusion: There is a paucity of well-designed clinical studies for patients who sustained mTBI. Accordingly, no intervention has been clearly associated with a positive outcome for these patients. The large variability in measured outcomes limits any meaningful comparison of studies. 
OP 18 National survey of emergency physicians for TIA risk stratification consensus and appropriate treatment for a given level of risk

J.J. Perry, MD, MSc, CCFP(EM), J. Losier, BHSc, J. Sutherland, BSc, MEd, I.G. Stiell, MD, MSc, FRCPC, M. Sharma, MD, MSc, FRCPC; University of Ottawa

Keywords: decision rules, emergency medicine, stroke, transient ischemic attack

Introduction: Five percent of transient ischemic attack (TIA) patients have a subsequent stroke $\leq 7$ days. The Canadian TIA Score uses clinical findings of ED TIA patients to calculate the subsequent stroke risk $\leq 7$ days. Our objectives were to assess (1) anticipated use; (2) component face validity; (3) risk strata for stroke $\leq 7$ days; and (4) actions required, for the Canadian TIA Score. Methods: We conducted a mail survey of 246 Canadian ED physicians listed in Scott's Canadian Medical Directory via a modified Dillman technique. We used prenotification, up to three survey attempts by letter mail (with a $\$ 10$ gift card), and a fourth by registered mail. We asked 41 questions, including demographics; face validity of the Score's components (4-point scale); if physicians will use the Score (4 point scale); cutoffs (open percents) for minimal, low, high, and critical risk; and suggested actions for each risk stratum (4-point scale). We calculated descriptive statistics. Results: Response rate was $49.5 \%$ (109/240). Respondents were males $74.3 \%$ with a mean age of 46.2 years. Respondents rating components as "very important" or “important" were first TIA (80.0\%), $\geq 10$ minutes' duration $(97.2 \%)$, history of carotid stenosis $(88.0 \%)$, antiplatelet therapy $(86.2 \%)$, gait disturbance $(76.9 \%)$, unilateral weakness $(97.2 \%)$, vertigo (- predictor) $(27.8 \%)$, speech disturbance $(96.2 \%)$, diastolic BP $\geq 110$ (71.4\%), atrial fibrillation (AF) on ECG (98.2\%), infarction on CT $(88.9 \%)$, platelets $\geq 400 \times 10^{9} / \mathrm{L}(25.2 \%)$, and glucose $\geq 15 \mathrm{mmol} / \mathrm{L}$ (36.1\%); $96.4 \%$ would use the Canadian TIA Score after validation. Using the 25 th percentile (i.e., $75 \%$ of physicians would accept this degree of risk or more) defined minimal risk as $<1 \%$, low risk $1-4.9 \%$, high risk $5-10 \%$, and critical risk $>10 \%$ for subsequent stroke $\leq 7$ days. Suggested actions were minimal risk: ECG in ED, CT/outpatient specialist follow-up, and ASA; low risk: ECG/CT/ cardiac monitoring in ED, ASA or warfarin (AF), and carotid imaging/echocardiogram/outpatient specialist follow-up; high and critical risk: ECG/CT/carotid imaging/echocardiogram/consultation/ASA or warfarin (AF), and admission. Conclusion: ED physicians will likely use a validated Canadian TIA Score. Most components have high face validity. Risk strata are definable with prescribed actions in ED. Care for minimal-risk patients $(<1 \%$ risk $)$ included ECG, ASA treatment, and outpatient $\mathrm{CT} /$ follow-up, compared to high-risk (5-10\% risk)/critical risk ( $>10 \%$ risk) included extensive investigations and admission.

OP19 A before-after study comparing complications of warfarin reversal using frozen plasma versus Octaplex

M. Hickey, MD, M. Gatien, MD, A. Aujnarain, MSc, A. Giulivi, MD, J.J. Perry, MD, MSc, CCFP(EM); University of Ottawa

Keywords: anticoagulation reversal, critical care, emergency medicine, hemorrhage

Introduction: Emergency physicians are often required to reverse patients' warfarin anticoagulation either with frozen plasma or prothrombin complex concentrate, including Octaplex. The objective of this study is to examine the efficacy and adverse effects of urgent reversal of anticoagulation using frozen plasma versus Octaplex in emergency department patients. Methods: This before-after study was conducted using health records of patients who were treated with frozen plasma or Octaplex for emergency reversal of anticoagulation in two tertiary emergency departments (ED). The before period was the 2 years prior to September 2008, at which time the routine management was frozen plasma; the after period was the 2 years after September 2008 when Octaplex was substituted by the blood bank for urgent reversal of patients with elevated INR due to warfarin therapy. We enrolled patients over 18 years, were taking warfarin with an INR $\geq 1.5$, and were ordered to receive frozen plasma or Octaplex in the ED. The primary outcome, serious adverse events, was a composite outcome of death, stroke, myocardial infarction, heart failure, venous thromboembolism, or peripheral arterial thromboembolism within 7 days. Secondary outcomes included time to INR reversal, mean hospital length of stay, and number of units of packed red blood cells transfused within 48 hours. Results: We included 149 patients in the frozen plasma group and 165 patients in the Octaplex group for analysis. For our primary outcome, there were serious adverse events in $19.5 \%$ of the frozen plasma group and $9.7 \%$ in the Octaplex group $(p=0.014)$. Time to INR reversal was 14.2 hours in the frozen plasma group and 8.9 hours in the Octaplex group $(p<0.001)$. The differences in mean hospital length of stay and red cell transfusion requirement were insignificant. Conclusion: This study suggests that Octaplex administration for emergency reversal of warfarin-induced anticoagulation may be associated with fewer adverse events than that of frozen plasma and should continue to be considered as a first-line treatment in this context.

$O P 20$ Implementation of the revised provincial acute Stroke Redirect Protocol in urban and rural settings

I.G. Stiell, MD, MSc, FRCPC, K. Smaggus, PCP, C.M. Clement, RN, M. Sharma, MD, MSc, FRCPC, D. Socha, BSc, PCP, M. Sivilotti, MSc, MD, FRCPC, A. Jin, MD, J.J. Perry, MD, MSc, CCFP(EM), J, Lumsden, BScPT MPA, C. Martin, BScPT, MSc, M. Froats, MD, R. Dionne, MD, CCFP(EM), J. Trickett, RN, BScN; Ottawa Hospital Research Institute

Keywords: emergency medicine, EMS, stroke

Introduction: The provincial Stroke Redirect Protocol was recently revised to allow EMS to bypass to designated stroke centres if total transport time would be $<2$ hours and total time from symptom onset $<3.5$ hours. We sought to evaluate the impact, effectiveness, and safety of implementing the revised Protocol within a large urban and rural region. Methods: We conducted a 12-month multicentre, prospective cohort study involving all prehospital patients presenting $<$ 6 hours with possible stroke symptoms. Participating were 1,000 BLS and 300 ALS paramedics of nine land EMS services, operating in a catchment area of 10 rural counties and 5 cities (total population of 1.7 million, total area 15,000 square miles), with 22 acute care hospitals and 2 university hospital stroke centres. Paramedics completed a record form for each case, and, initially, a second paramedic independently completed the form. Outcomes and data analyses included redirect sensitivity and specificity, patient outcomes, adverse events, and interrater reliability with the kappa statistic. Results: We enrolled 1,277 eligible patients with $99 \%$ paramedic compliance in form completion. Of these, $755(61.2 \%)$ met the redirect criteria and had these characteristics: mean age 72.0 (range 16-101), male 51.1\%, mean time scene to hospital 16.7 minutes (range $0-92$ ) with $15.1 \%>30$ minutes. The prehospital adverse event rate was $14.7 \%$ (23.0\% for those with transport time $>30$ minutes), with the most common events being hemodynamic instability and drop in GCS. At the hospital, the 755 patients had a mean NIH Stroke Scale score of 8.7, 23.8\% received thrombolysis, $69.3 \%$ were admitted, $87.3 \%$ survived to discharge, and had a mean modified Rankin Score of 2.3. Paramedics showed 97.9\% accuracy in interpreting the criteria and excellent interrater agreement, with kappa values ranging from 0.56 to 0.90 for redirect criteria and 0.94 for need to transport to a stroke centre. The positive predictive 
values for the Protocol were stroke code activation $71.8 \%$, thrombolysis $23.2 \%$, admitted $69.3 \%$, and final diagnosis stroke/TIA $70.6 \%$. Including all patients assessed within one city, the Protocol classified need for thrombolysis with sensitivity $100 \%$ and specificity $37.3 \%$. Conclusion: In a large urban-rural area with nine EMS services, we demonstrated accurate, safe, and effective implementation of the revised provincial Stroke Redirect Protocol. These revisions will allow more stroke patients to benefit from early treatment.

$O P 21$ ACES high: how realistic is high-fidelity ultrasound simulation for abdominal and cardiothoracic assessment with sonography in shock?

P.R. Atkinson, MD, FCEM, G. Verheul, MD, J. Fraser, Bachelor of Nursing, D. Lewis, MBBS, FRCS, FCEM; Discipline of Emergency Medicine, Memorial University

Keywords: emergency medicine, point-of-care ultrasound, simulation Introduction: Shock ultrasound training can be limited by the availability of patients with pathology. Simulation may provide a more efficient way of training. We assessed high-fidelity ultrasound simulation for how realistic it appeared for normal anatomy and pathology seen when performing Abdominal and Cardiothoracic Evaluation by Sonography in shock (ACES) scans. Methods: Forty-two physicians participated in educational sessions that included didactic learning followed by supervised scanning normal volunteers and patients with pathology using portable ultrasound machines (GE \& SonoSite). Participants performed focused ACES scans using a high-fidelity ultrasound simulator (VIMEDIX), imaging the heart, chest, abdomen, aorta, and inferior vena cava (IVC). All participants were then surveyed using a standardized 5-point Likert scale for how realistic normal anatomy and pathology appeared. Data were analyzed using ANOVA (GraphPad Prism). Results: All 42 surveys were completed. Twenty-seven (64.3\%) had previous ultrasound training, and two $(4.8 \%)$ had previously used the ultrasound simulator. The simulator was rated as realistic to very realistic for real-time demonstration of cardiac (mean score $4.3 ; 95 \% \mathrm{CI}$ $4.0-4.5)$, chest $(4.2 ; 3.9-4.4)$, abdominal $(4.1 ; 3.9-4.3)$, aortic $(4.1$; $3.8-4.4)$, and IVC $(4.2 ; 4.0-4.5)$ pathology and for normal cardiac (4.2; 4.0-4.5), chest (4.2; 4.0-4.5), abdominal (4.1; 3.9-4.4), aortic (4.1; 3.9-4.4), and IVC $(4.3 ; 4.1-4.5)$ anatomy. Forty-one (97.6\%) participants stated that they would use high-fidelity ultrasound simulation to further develop and maintain skills with trauma ultrasound. All simulation groups scored significantly better than neutral ("neither realistic or nonrealistic"; $p<0.05)$. Conclusion: Simulated ultrasound was rated as realistic for normal anatomy and pathology for all ACES windows. It is likely that ultrasound simulation will have an increasingly important role in shock ultrasound education and skills maintenance, although it is unlikely to replace the need for training on live patients.

$O P 22$ Validation of a tool for the assessment of trainees during simulated pediatric resuscitation

Y. Shayan, MDCM, J. Gravel, MD, MSc, F. Bhanji, MD, MSc, S. Manzano, MD, O. Karam, MD, M.J. Weiss, MD, FRCPC, FAAP, I. Bank, MDCM, FRCPC, FAAP, G. Choker, MD, A. Levy, MD; Hôpital Sainte-Justine

Keywords: emergency medicine, evaluation, simulation

Introduction: Recently, Grant et al developed a tool that assesses clinical performance and leadership skills during simulated pediatric resuscitations. We aimed to evaluate external validity and interrater reliability of Grant's assessment tool in a setting other than where it was developed. Methods: This project took place in the simulation lab of a tertiary care pediatric centre. In the setting of a previous study, 13 firstyear and 11 third-year residents were videotaped during simulated pediatric resuscitation scenarios. Each resident led five scenarios before and after their Pediatric Advanced Life Support (PALS) course. The pre- and post-PALS scenarios were paired such that each resident acted as team leader in a pulseless nonshockable arrest, pulseless shockable arrest, dysrhythmia, respiratory and shock scenario. Five specialists in the fields of pediatric emergency medicine and intensive care from North America and Europe were trained to evaluate the residents' performance using Grant's assessment tool. Raters were blinded to the pre- and post-PALS phases of the scenarios. Interrater reliability was assessed by having two expert raters independently evaluate the residents' performance. In the absence of a gold standard scoring tool, we used construct validity to validate the tool. Knowing that the PALS course has been shown to increase immediate short-term knowledge of health care professionals, it was determined that, for the tool to be valid, participants should improve their score after participating in the PALS course. Pre- and post-PALS scores were compared using a paired-sample Student $t$-test. Interrater reliability was measured for the five scenarios using intraclass correlation coefficient (ICC). Results: Following the PALS course, the score improved by $11 \%(6.1-16)$ for the pulseless nonshockable arrest, $17 \%(11-24)$ for the pulseless shockable arrest, $6 \%(0.1-12)$ for the dysrhythmias, $12 \%(4.3-21)$ for the respiratory scenario, $14 \%(7.3-21)$ for the shock scenarios. Interrater reliability was excellent as demonstrated by an overall ICC of 0.91 (95\% CI 0.88-0.93). Conclusion: Grant's scoring instrument demonstrated construct validity and excellent reliability as a measure of clinical performance for simulated pediatric resuscitations. We were also able to show that it is a generalizable tool as it was valid for all of our five simulated scenarios.

OP23 The sleep habits of junior residents and medical students working in the emergency department

D.J. Ginsberg, MSc, M.L. Sivilotti, MD, MSc, A.K. Hall, MD, J.D. Dagnone, MD; Queen's University

Keywords: clinical reasoning, education, emergency medicine, sleep habits, teaching

Introduction: Shiftwork, routine in emergency medicine (EM), has various negative influences on sleep quality and cognitive function due to disruption of the natural circadian rhythm. Despite increasing attention on trainee work schedules and hours of continuous and cumulative duty, there are few data on the sleep habits of residents and medical students working in EM and how these traits influence clinical abilities. Methods: In a feasibility study, junior residents and medical students working in EM in Kingston, Ontario, during August-October 2012 completed validated questionnaires to determine their chronotype (Eveningness-Morning Questionnaire), daytime sleepiness (Epworth Sleepiness Scale), and sleep quality (Pittsburgh Sleep Quality Index). At weekly intervals, sleep logs, the Karolinska Sleepiness Scale, and the Mental Fatigue Scale were collected prior to participants viewing a 4-minute simulated EM case. Their written management was graded (0-100) using a global rating scale by two independent blinded evaluators. Results: Thirty-four participants (8 PGY1-2 EM residents, 11 offservice residents, and 15 medical students) consented to the study and provided data on 2.1 sessions/participant. While total sleep per day (overall mean $\pm \mathrm{SD}=8.5 \pm 1.2$ hours) was similar between groups, $\mathrm{EM}$ residents tended more toward an evening chronotype than off-service residents and students, scoring $16.5 \pm 5.5$ versus $20.6 \pm 4.9$ out of $32(p<0.05)$. An orientation toward an evening chronotype was correlated with longer sleep duration $(R=-0.66 ; p<0.01)$. More senior trainees reported poorer quality sleep $(R=0.71 ; p<0.01)$. A noncircadian sleep $(<4$ hours of sleep between 00:00 and 0:600) within the previous 4 days, as reported by $38 \%$ of participants, was associated with reduced sleep $(7.9 \pm 1.4$ v. $8.8 \pm 1.3$ hours $)$, greater sleepiness $(6.5 \pm$ 
1.7 v. $4.7 \pm 3.2$ out of 9$)$, and fatigue $(6.4 \pm 2.2$ v. $4.8 \pm 2.4$ out of 9 ; all $p<0.01)$. Global blinded evaluation scores had a strong interobserver correlation $(R=0.84 ; p<0.01)$ and were substantially different between groups $(\mathrm{EM}$ residents $=56.8 \pm 19.7 \mathrm{v}$. off-service residents $=$ $39.3 \pm 18.4$ v. students $=28.3 \pm 14.8 ; p<0.01)$. Conclusion: Noncircadian sleep is common in trainees during an EM rotation and leads to reduced sleep and increased fatigue. This study's innovative method can discriminate performance by training level and is feasible to scale up to a larger sample to determine how shiftwork affects clinical reasoning in EM.

OP24 Prospective evaluation of a 20-metre walk test in elderly emergency department patients

A. Hendin, BASc, C.M. Clement, RN, I.G. Stiell, MD, MSc, FRCPC; University of Ottawa, Department of Emergency Medicine

Keywords: discharge, elderly, walk test

Introduction: Elderly patients will make up an increasing number of emergency department (ED) visits as the Canadian population ages; this group is at high risk for ED revisit following discharge. This study aimed to evaluate the feasibility and usefulness of a 20 -metre walk test, performed in the ED, in predicting adverse outcomes among elderly patients being considered for discharge. Methods: We conducted a prospective cohort study in the ED of a Canadian tertiary care hospital over a 3-month period and enrolled a consecutive sample of patients 65 years of age and older who were seen in the nonambulatory areas. We excluded patients who were unstable at the time of assessment, had a leg injury, or were in a chronic care facility/nursing home. With a research assistant, subjects walked 20 metres and had their oxygen saturation and heart rate measured continuously during the walk. Follow-up after a 14day period was completed to record discharge status and patient outcomes (adverse outcomes included admission to hospital, return to ED, death). Data were analyzed with descriptive and univariate tests. Results: Sixty subjects (53.3\% female) were enrolled with a mean age of 78.8 years (range 65-93) and mean CTAS score 2.5 (range 2-3). The most common presenting complaints were chest pain (18.3\%), dyspnea (15.0\%), and dizziness (11.7\%). Forty-four patients (73.3\%) completed the walk test with a mean time to completion of 49.7 seconds (range 13$185)$. Another 16 subjects $(26.7 \%)$ failed to complete the test due to $\mathrm{O}_{2}$ saturation $<90 \%(50.0 \%)$, unable to stand unassisted $(25.0 \%), \mathrm{HR}>110$ $(18.8 \%)$, or leg weakness $(6.3 \%)$. Overall, $81.7 \%$ of patients were discharged from the ED, and of those, $5(8.3 \%)$ returned to hospital within 14 days and $2(3.3 \%)$ were admitted. The patients who failed the walk test were more likely to be female $(75.0 \%$ v. $45.5 \%)$, to have a higher mean maximum heart rate $(93.7$ v. 81.3$)$, to have a lower minimum $\mathrm{O}_{2}$ saturation ( 89.5 v. 95.1$)$, and to be admitted (37.5\% v. $11.4 \%$ ) (all $p$ values $<0.05$ ). A successful walk test predicted ED discharge with sensitivity $88.6 \%(95 \%$ CI $76.0-95.1)$ and specificity $37.5 \%$ (18.5-61.4). Conclusion: The 20-metre walk test is a practical and quick way to evaluate functional mobility in elderly ED patients being considered for discharge. Larger studies should evaluate the usefulness of the walk test in predicting clinical and functional outcomes.

$O P 25$ Correlates of $\mathbf{3 0}$-day adverse events for ED patients with recent-onset atrial fibrillation and flutter (RAFF)

I.G. Stiell, MD, MSc, FRCPC, C.M. Clement, RN, R.J. Brison, MD, FRCPC, E. Lang, MD, B.H. Rowe, MD, MSc, CCFP(EM), B. Borgundvaag, PhD, MD, D. Birnie, MD, J.J. Perry, MD, MSc, CCFP(EM), D. Eagles, MD, P. Dorian, MD, D.P. Redfearn, MD, G. Wyse, MD, G.A. Wells, PhD; University of Ottawa, Department of Emergency Medicine

Keywords: atrial fibrillation, emergency medicine, outcomes, quality of life
Introduction: Recent-onset atrial fibrillation and flutter (RAFF) are the most common arrhythmias managed in the ED. Our goal was to explore the association of patient factors and ED treatment strategies with 30-day patient outcomes. Methods: We conducted a prospective cohort study in the EDs of six large, tertiary care Canadian hospitals and enrolled adult RAFF patients who had clear onset within 48 hours. The primary outcome, adverse event (AE), was defined as any of the following within 30 days of ED discharge: death, stroke, acute coronary syndrome, heart failure, admission to hospital for RAFF, or return to the ED for RAFF. By health records review and patient telephone follow-up at day 45, we determined clinical outcomes and AEs. We assessed the association of patient and treatment variables with AE using univariate and mulitivariate logistic regression analyses. Results: We enrolled 1,091 patients: mean age 64.0 (range 16-103), male $59.5 \%$, initial rhythm atrial fibrillation $84.7 \%$ and atrial flutter $15.3 \%$, mean duration symptoms 7.7 hours, mean heart rate 122.7 , prior RAFF $65.0 \%$, prior electrical cardioversion $32.2 \%$, median CHADS2 score 1 (IQR 0-2), admitted to hospital 9.0\%, and converted to sinus rhythm $80.1 \%$. Sites varied for initial treatment strategy $(p<0.001)$, with overall averages being rate control only $17.8 \%$, observe only $9.4 \%$, rhythm drug first $33.7 \%$, electrical shock first $39.1 \%$. The 30 -day AE rate was $17.0 \%$ : death $0.3 \%$, stroke $0.1 \%$, acute coronary syndrome $0.6 \%$, heart failure $0.9 \%$, admitted $3.3 \%$, return to $\mathrm{ED} 15.4 \%$. Adjusted odds ratios for factors independently associated with AE were past history of stroke or TIA 1.9 (95\% CI 1.1-3.4), heart failure on chest x-ray 4.3 (1.7-10.4), hours from onset per hour 1.02 (1.0-1.03), discharged home 2.6 (1.3-5.4), and not converted to sinus rhythm at ED discharge 1.7 (1.2-2.6) (HosmerLemeshow goodness-of-fit 0.74). Conclusion: While 17\% of RAFF patients had AEs within 30 days of their ED visit, there were few related deaths or strokes. We identified a number of factors associated with 30-day AEs, including lack of rhythm control in the ED. ED physicians should consider these factors to ensure the optimal management and follow-up for RAFF patients.

$O P 26$ Evaluation the effect of emergency department crowding on triage decision making and patient outcomes

E. O'Connor, MD, MSc, L.A. Calder, MD, MSc, FRCPC, M. Gatien, MD, C. Weir, RN; University of Ottawa, Department of Emergency Medicine

Keywords: clinical decision making, emergency medicine, overcrowding, quality improvement

Introduction: Emergency departments are increasingly operating under conditions of crowding. We aimed to study patients presenting with chest pain or shortness of breath and triaged as 2 (Emergent) on the Canadian Triage and Acuity Scale (CTAS). Specifically, we sought to determine whether (1) patients were triaged to nonmonitored areas of the ED more frequently during crowding; (2) patients were seen more quickly in nonmonitored rather than monitored areas; (3) the proportion of return ED visits was higher for patients triaged during crowding; (4) the proportion of return ED visits was higher for patients triaged to the nonmonitored area. Methods: This study was a retrospective chart review of 500 patients presenting to an urban tertiary care ED with chest pain or shortness of breath and triaged as CTAS 2. Data extracted included triage time and date, time to physician assessment, disposition, and return visits within 14 days. We defined crowding as ED occupancy (the ratio of patients to beds in the ED) greater than 1.5. We analyzed the data with descriptive statistics and chi-square testing. Results: We reviewed 500 charts, and of these, $392(78.4 \%)$ patients presented with chest pain, 108 (21.6\%) presented with shortness of breath, and $260(52.0 \%)$ presented during conditions of crowding. More patients were triaged to the nonmonitored area of the ED when the ED was crowded (65/260 [25.0\%] v. 
$39 / 240$ [16.3\%]; when not crowded, $p=0.016)$. When the ED was crowded, mean time to initial physician assessment was 132.0 minutes in the nonmonitored area, compared to 99.1 minutes in the monitored area, $p<0.0001$. When the ED was not crowded, mean time to physician initial assessment was 122.3 minutes in the nonmonitored area and 67 minutes in the monitored area, $p=0.0003$. Patients did not return to the ED more often when triaged during conditions of crowding $(9.3 \%[n=24]$ of patients triaged when the ED was crowded returned within 14 days of the index visit v. $12.1 \%[n=29]$ when ED not crowded $[p=0.31])$. Overall, when triaged to the monitored area of the ED, $11.1 \%(n=44)$ of patients returned, compared to the nonmonitored area, which had $8.7 \%$ of patients $(n=9)$ return. Conclusion: Crowding conditions appeared to influence triage decisions and destination in our emergency department, leading to longer wait times for patients with chest pain and shortness of breath. This did not, however, appear to lead to higher rates of return ED visits among discharged patients in this cohort.

OP27 The efficacy of pad placement for electrical cardioversion of atrial fibrillation: a systematic review

S. Kirkland, BSc, MSc, I.G. Stiell, MD, MSc, FRCPC, T.Y. AlShawabkeh, MD, JBEM, S. Campbell, BA, MLS, G. Dickinson, MD, FRCPC, FIFEM, B.H. Rowe, MD, MSc, CCFP(EM); University of Alberta

Keywords: atrial fibrillation, cardioversion, efficacy, emergency medicine

Introduction: Electrical cardioversion (EC) is commonly used to treat patients with atrial fibrillation and flutter (AFF) to restore normal sinus rhythm (NSR). There has been considerable debate as to whether the electrode placement impacts the efficacy of EC. The objective of this study was to examine the effectiveness of anteroposterior (A-P) versus anterolateral (A-L) electrode placement to restore NSR. Methods: A search of eight electronic databases, including Medline, EMBASE, CINAHL, and Cochrane, was completed. Grey literature (hand searching, Google, and SCOPUS) searching was also conducted. Studies were included if they were controlled clinical trials comparing the effectiveness of A-P versus A-L pad placement to restore normal sinus rhythm in adult patients with AFF. Two independent reviewers judged study relevance, inclusion, and quality (e.g., risk of bias $[\mathrm{RoB}])$. Individual and pooled statistics were calculated as relative risks (RR) with $95 \%$ confidence intervals (CI) using a fixed effects model and heterogeneity $\left(\mathrm{I}^{2}\right)$ was reported. Results: From 788 citations, 13 studies were included; 7 involved monophasic, 5 involved biphasic, and 1 analyzed both waveform devices. The included studies tended to report cumulative success rates to restoring NSR after one to five sequential shocks of increasing energy; the number of shocks and energy used differed among studies. The RoB of the studies was "unclear." After the first shock, pad placement was not associated with an increased likelihood of restoring NSR (RR = 0.88 ; $95 \%$ CI: $0.73,1.06)$; however, heterogeneity was high $\left(\mathrm{I}^{2}=\right.$ $63 \%$ ). Subgroup comparisons revealed that A-P position was less effective $(\mathrm{RR}=0.77 ; 95 \% \mathrm{CI}: 0.59,1.00)$ at restoring NSR when using biphasic shocks (comparison $p=0.04$ ). Overall, the pooled results failed to identify a difference between A-P and A-L pad placement in restoring NSR at any time $(\mathrm{RR}=1.00 ; 95 \% \mathrm{CI}: 0.95,1.05)$; however, heterogeneity was high $\left(\mathrm{I}^{2}=61 \%\right)$. No significant subgroup differences were found. Side effects were reported in only three studies. Conclusion: The accumulated evidence suggests that electrical pad placement is not a critically important factor in successful cardioversion of atrial fibrillation; however, A-L placement for the initial shock appears to be more effective using biphasic devices. Other factors (e.g., chest impedance, duration, voltage) likely play more important roles in successful EC.
$O P 28$ Clinical and laboratory findings to differentiate herpes simplex from enteroviral meningitis

L. Sanaee, MD, T. Karnauchow, MD, J.J. Perry, MD, MSc, CCFP(EM); University of Ottawa

Keywords: emergency medicine, infection, lumbar puncture, neurology

Introduction: In contrast to relatively common enteroviral (EnV) meningitis, early recognition of herpes simplex (HSV) meningitis is considered essential as early treatment can reduce mortality by 20 to $30 \%$. Since currently there is no objective way to promptly differentiate benign EnV from the potentially life-threatening HSV meningitis, this study aims to identify clinical features and cerebrospinal fluid (CSF) results that differentiate the two. Methods: We conducted a historical cohort study from January 2005 to December 2011, enrolling patients greater than 3 months of age with HSV or EnV polymerase chain reaction (PCR)-positive CSF specimens, identified at our regional virology laboratory. Patients without PCR results who had other CSF findings and neuroimaging consistent with viral meningitis and a final diagnosis of meningitis were included. Clinical and laboratory data were obtained from electronic records and paper charts, including emergency department triage assessments, records of treatment, and discharge summaries. Descriptive and univariate statistical analyses were conducted for clinical features and CSF findings. Results: A total of 166 consecutive patients were enrolled in the study, with $40 \mathrm{HSV}$ and $126 \mathrm{EnV}$ cases. A significant difference between HSV and EnV patients was found in mean age (40.4 v. $31.3, p=$ $0.005)$, history of seizure $(21.1 \mathrm{v} .1 .6 \%, p=<0.001)$, history of altered mental status (46.2 v. $3.2 \%, p=<0.001)$, and neurologic deficit on physical examination ( 44.7 v. $3.9 \%, p=<0.001)$. CSF findings demonstrated no significant difference in white blood cell counts $\left(331.1 \times 10^{6}\right.$ v. $\left.279.2 \times 10^{6} / \mathrm{L}, p=0.5\right)$; however, neutrophil differential counts were significantly lower in HSV cases $(7.2$ v. $23.7 \%, p=<$ $0.001)$, and lymphocyte counts $(79.7$ v. $61.6 \%, p=<0.001)$ and protein levels $(1.0$ v. $0.7 \mathrm{~g} / \mathrm{L}, p=0.03)$ were significantly higher. Subgroup analyses of PCR confirmed $(n=29 \mathrm{v} .19)$ and immunocompetent confirmed cases $(n=23 \mathrm{v}$. 19) revealed consistent findings. Conclusion: In this study, patients with HSV meningitis were more likely than EnV meningitis to be older; present with seizure, altered mental status, and neurologic deficits; and have lower CSF neutrophil counts and higher CSF lymphocyte and protein levels. HSV treatment should be strongly considered in patients with one or more of these features while awaiting definitive PCR results.

OP29 A prospective evaluation of the treatment preferences of ED patients with recent-onset atrial fibrillation and flutter (RAFF) J.H. Yoo, BSc, D. Eagles, MD, C.M. Clement, RN, I.G. Stiell, MD, MSc, FRCPC; University of Ottawa, Department of Emergency Medicine

Keywords: atrial fibrillation, emergency medicine, shared decision making, treatment preferences

Introduction: Recent-onset atrial fibrillation (AF) and flutter (AFL) (RAFF) is the most common arrhythmia managed in the ED. Patients increasingly expect to be involved in decision making about their own medical care. The purpose of this study was to evaluate the treatment preferences of ED patients with RAFF. Methods: We conducted a multicentre cohort study in the EDs of six tertiary care hospitals and enrolled adult patients presenting with RAFF. The self-completed questionnaire asked patients to (1) rate the importance of these issues from 1 to $5(1=$ not important, 5 = very important $)$ : restoration of sinus rhythm, expedient care, avoidance of side effects, avoidance of electrical cardioversion (ECV), least aggressive initial treatment, and knowledge of their arrhythmia and options; (2) indicate their treatment preference; and (3) report their desired level of involvement in treatment 
decisions. They also completed the previously validated "ED RAFF Scale" and the SF-8 Health Survey. Descriptive and univariate analyses were used. Results: We enrolled 82 patients: mean age 64.2 years, male $47.0 \%$, rhythm AF $78.0 \%$ and AFL $22.0 \%$, mean duration of symptoms 8.4 hours, mean heart rate 123.1 , prior RAFF $53.7 \%$, prior ECV $25.6 \%$, prior pharmacologic cardioversion (PCV) $11.0 \%$, and median CHADS2 score 1 (IQR 0-2). The proportion of patients ranking each item as important or very important (scores of 4-5) was restoration of sinus rhythm $87.8 \%$, expedient care $69.5 \%$, avoidance of side effects $73.2 \%$, avoidance of ECV $34.2 \%$, least aggressive initial treatment $52.4 \%$, and knowledge of their arrhythmia and options 98.8\%. Patients preferred cardioversion $46.3 \%$ (ECV $32.9 \%$ v. PCV $13.4 \%$ ) over rate control $12.2 \%$, whereas $31.7 \%$ had no preference. The median desired decision-making involvement was 50\% (IQR 50$60 \%$ ). No differences were seen between the rated importance of issues and desired involvement when comparing prior RAFF patients with new-onset RAFF patients and high symptom severity patients with mild to moderate symptom severity patients. Conclusion: This is the first study to evaluate patient preferences in management of RAFF. Patients placed high importance on restoration of sinus rhythm and knowledge of their condition and treatment options, whereas avoidance of ECV was the least important. Patients clearly desired shared decision making in the treatment of RAFF, and the results of this study will help guide future management strategies for RAFF in the ED.

OP30 Can medical learners achieve ultrasound competency using a high-fidelity ultrasound simulator?

A. Parks, Medical Student, P.R. Atkinson, MD, FCEM, G. Verheul, MD; Dalhousie Medicine, New Brunswick

Keywords: emergency medicine, medical education, simulation Introduction: Point-of-care ultrasound (PoCUS) is currently not a universal component of curricula for medical undergraduate and postgraduate training. We designed and assessed a simulation-based PoCUS training program for medical learners, incorporating image acquisition, interpretation, and clinical integration using simulated emergency medical scenarios. We wished to see if learners could achieve competency following training in a focused PoCUS protocol. Methods: Twelve learners (clerks and residents) received standardized training consisting of online preparation materials, didactic teaching, and an interactive hands-on workshop using a high-fidelity mannequin simulator (SimMan 3G) and a high-fidelity ultrasound simulator (CAE Vimedix). We used the Abdominal and Cardiothoracic Evaluation by Sonography (ACES) protocol as the curriculum for PoCUS training. Participants were assessed during 72 simulated emergency cardiorespiratory scenarios. Their ability to complete an ACES scan independently was assessed. Differential diagnoses were collected from participants before and after PoCUS simulator incorporation in each scenario. Data were analyzed using GraphPad Prism. Results: Participants independently generated and interpreted $574(99.7 \%)$ of the 576 expected ultrasound windows during the 72 simulated scenarios. Learners demonstrated their competency by improving their clinical diagnostic accuracy from $45 / 72(62.5 \%)$ to $64 / 72(88.9 \%)$ correct primary diagnoses when using PoCUS; by increasing their diagnostic confidence from a mean pre-PoCUS confidence score of $52.2(\mathrm{SD}=14.7)$ to 81.7 $(\mathrm{SD}=9.5)$ with PoCUS $(t=-7.71, p<0.0001)$; and by narrowing their differential-diagnosis from a median of $3.5(\mathrm{IQR}=[3.0,3.8])$ diagnoses per patient pre-PoCUS, to $2.3(\mathrm{IQR}=[1.5,2.9])$ post-PoCUS $(\mathrm{W}=0$, $p<0.001)$. Conclusion: We have demonstrated that after a focused training process using medical simulators, medical learners demonstrated an ability to achieve a degree of competency to both acquire and correctly interpret and integrate common cardiorespiratory PoCUS findings using a high-fidelity ultrasound simulator.
$O P 31$ Children's satisfaction with pain management in the emergency department

L. Weingarten, MD, S. Ali, MDCM, A. Newton, PhD, RN, A. Drendel, DO, MS; Department of Pediatrics, University of Alberta

Keywords: emergency medicine, pain, pediatrics, satisfaction Introduction: Pain accounts for nearly $80 \%$ of emergency department (ED) visits and is the most common reason for seeking health care. Although children most accurately report their own pain, the majority of studies ask third parties (e.g., physicians, nurses, and caregivers) how well a child's pain has been managed. This project surveyed children directly to examine their satisfaction with pain management in the ED. Methods: This was a prospective, descriptive, cross-sectional study. One hundred children, aged 7-17 years, were recruited from October 2011 to November 2012. Assent and consent were obtained. Trained research assistants verbally administered the Child Total Quality Pain Management Questionnaire, a validated tool to measure children's satisfaction with pain management. They also collected demographic information including the child's age, sex, and physician/nurse discharge advice. Results: One hundred children participated; $47 \%$ were female. The average age was 12.6 years (range $7-16$ ). Mean pain duration was 3.6 days. Most children had injuries of the upper and lower limbs ( $n=31$ and 26, respectively). Other presenting problems included head/neck $(n=19)$, abdominal $(n=9)$, back/shoulder $(n=6)$, and other types $(n=9)$ of pain. The average maximum pain reported was $79 \mathrm{~mm}(95 \%$ confidence interval $[\mathrm{CI}] 75,82)$, and average pain reported at discharge was $34 \mathrm{~mm}(95 \%$ CI 29,39$)$ on a $100 \mathrm{~mm}$ pain scale. Interval improvement in pain was $45 \mathrm{~mm}(95 \%$ CI 39, 51). Forty-two children were "very happy" with their pain management, 50 were "happy," and 7 were "unhappy"/"very unhappy"; $87 \%$ would want the same medicine again. Conclusion: Children present with and continue to experience severe pain in the ED. Although many children have significant pain upon discharge, most report a high degree of satisfaction with their pain management. The relationship between satisfaction and pain management should be explored further as children's satisfaction does not necessarily correlate with adequate analgesia. Physicians must be careful not to interpret patient satisfaction as adequate pain management.

$O P 32$ Feasibility of implementing an adverse drug event screening program in an emergency department

C.M. Hohl, MDCM, FRCP, MHSc, L.N. Reddekopp, Bachelor of Nursing, R. Tsang, Doctorate of Pharmacology, N. Partovi, Doctorate of Pharmacology, E. Yu, MS, J. Coleman, Doctorate, A. Karpov, Doctorate, D. Campbell, MBA; University of British Columbia

Keywords: emergency medicine, medication review

Introduction: Adverse drug events (ADEs) account for 1.7 million emergency department (ED) visits in Canada annually. Yet ADEs are misdiagnosed in $40-50 \%$ of cases. Our objective was to determine the feasibility of implementing an ADE screening program in one tertiary care ED. Methods: This prospective demonstration project was conducted over a 12-month period in one tertiary care ED. The screening program had three components: (1) triage nurses screened incoming patients using a clinical decision rule to classify patients as high/lowrisk for ADEs; (2) pharmacists performed medication review in a systematically selected sample of high-risk patients; and (3) pharmacists communicated $\mathrm{ADE}$ information to treating physicians and general practitioners and documented them in a province-wide medication information system. We evaluated the program's feasibility by measuring its impact on triage times and pharmacist workload. We measured its uptake by clinicians by evaluating the proportion of incoming patients to whom the clinical decision rule was applied and the propor- 
tion of high-risk patients in whom medication review was completed. Results: During the 12-month demonstration project, 83,769 patients presented to the ED, of whom 82,910 (99.0\%) were screened for ADEs using the clinical decision. The median triage time was not increased by inclusion of the clinical decision rule (6.1 minutes pre v. 4.7 minutes post; difference 1.4 minutes, $95 \%$ CI $0.8-2.0$ ). Nurses classified $23,549(28.4 \%)$ patients as high risk with an accuracy of $86.0 \%(95 \%$ CI 81.7-89.5). During hours of ED pharmacist coverage (8-midnight weekdays and 1-9 pm weekends), pharmacists completed medication review in 1,335 (44.7\%) high-risk patients. The median time required to complete medication review was 21.4 minutes (95\% CI: 19.4-26.7). Conclusion: Implementing an ADE screening program consisting of an initial screen using a clinical decision rule followed by pharmacistled medication review was feasible with added clinical pharmacist hours. Further studies are needed to investigate the impact on health outcomes and cost.

OP33 Regional 30-day outcomes in patients presenting to the ED with suicidal ideation or overdose attempt

E. Grafstein, MD, R. Stenstrom, MD, PhD, J. Christenson, MD, G. Innes, MD, F.X. Scheuermeyer, MD; St. Paul's Hospital

Keywords: administrative database, emergency medicine, suicide, vital statistics

Introduction: Suicide after ED evaluation is devastating to families and ED staff. We studied the epidemiology of suicide in a health region with the primary outcome of 30-day mortality post ED visit. Methods: An administrative database review using the previously validated Vancouver Coastal Health $(\mathrm{VCH})$ region ED database (300,000 annual visits). All patients who presented to one of six $\mathrm{VCH}$ emergency departments with a presenting complaint of suicidal ideation, attempt, or overdose (OD) over a 48-month period between January 2008 and 2012 were studied. Visits were linked with provincial vital statistics data to identify deaths due to suicide or possible suicide within 30 days of ED visit. Possible suicides were adjudicated by a panel of ED experts from all causes of death in the study cohort (free marginal kappa $=89.1 \%$ ). We excluded patients without a provincial health number. Results: A total of 11,237 patients made 18,804 visits related to suicidal ideation/OD during the study period. The admission rate was $38.2 \%(7,189)$, and 42 patients $(0.37 \%)$ died within 30 days (all-cause mortality). Of these, 31/11,237 (0.28\%) were suicide or possible suicide, including 15 suicides $(0.13 \%), 8$ accidental overdoses $(0.07 \%)$, and 8 ill-defined deaths $(0.07 \%)$. Only 9 of these 31 patients were discharged home primarily $(0.08 \%)$. These 31 patients had an average of 7.7 visits (SD 8.3), and 11/31 visited more than one regional ED. Of the 15 definite suicides, only 3 were discharged home after their last ED visit (0.03\%). Eighteen patients who died within 30 days had an intervening ED visit with a nonsuicidal ideation/OD complaint. Conclusion: Very few patients with suicidal ideation commit suicide within 30 days. Most of those who committed suicide did so after formal hospitalization for suicidal ideation. Different treatment strategies that rely less on hospital admission may be possible. Analysis of risk factors associated with suicide is ongoing.

$O P 34$ A survey of factors associated with the use of intraosseous access in adult patients when peripheral intravenous access is not available

W. Cheung, MD, H. Rosenberg, MD, C. Vaillancourt, MD, MSc, FRCPC, CSPQ; University of Ottawa, Department of Emergency Medicine

Keywords: emergency medicine, intraosseous, resuscitation, vascular access
Introduction: Studies suggest that intraosseous (IO) access is underutilized despite recommendations from advanced trauma and cardiac life support guidelines. We sought to determine factors associated with IO use by physicians during adult resuscitations when intravenous (IV) access is not immediately achievable. Methods: We conducted an online survey among physicians purposefully recruited from various clinical care areas at two teaching hospitals. Questions were generated from the qualitative results of 20 iterative interviews, verified for internal validity, and piloted. The interview guide was based on the constructs of the Theory of Planned Behaviour (TPB), which elicits salient attitudes, social influences, and control beliefs potentially influencing intention to use an IO. Recruitment took place in September 2012 until reaching more than $100 \%$ of the required sample size $(N=200)$. We measured internal consistency using Cronbach's alpha and the effect of TPB constructs and specific beliefs with regression analyses. Results: Demographics of the 225 respondents: mean age $34.9 ; 46.7 \%$ female; $50.3 \%$ emergency medicine, $16.9 \%$ internal medicine, $14.9 \%$ anesthesia, $10.8 \%$ general surgery, $7.2 \%$ critical care; $60.7 \%$ residents versus $39.3 \%$ attendings. Median intention to use IO access when IV is not immediately achievable was $4.7 / 5$ ( 5 highest) and predicted by the following TPB constructs: attitudes (AdjOR 1.8; CI 1.5-2.1), social influences (AdjOR 1.4; CI 1.3-1.6), control beliefs (AdjOR 1.3; CI 1.11.4), and specific beliefs: $\mathrm{IO}$ is associated with low complication rates (OR 1.2; CI 1.1-1.3), nurses are not familiar with IO access and their use once inserted (OR 1.2; CI 1.1-1.2), and physicians need to understand the indications for inserting an IO (OR 1.1; CI 1.0-1.2). Conclusion: Educational interventions designed to inform nurses about the utility and use of IO access and to educate physicians of the indications and potential complications are most likely to increase IO use in adult resuscitations when IV access is not immediately achievable.

\section{OP35 The current use of bronchodilators and steroids for the management of bronchiolitis in Canadian pediatric emergency departments}

A. Plint, MD, R. Grenon, MA, T.P. Klassen, MD, D.W. Johnson, MD; University of Manitoba

Keywords: bronchiolitis, emergency medicine, pediatrics

Introduction: The American Academy of Pedatrics bronchiolitis practice guideline does not recommend routine bronchodilator or steroid use. One trial published since this guideline's development suggests a possible benefit of combined treament with epinephrine and dexamethasone. Given this trial, we reviewed the current management of bronchiolitis in Canadian pediatric emergency departments (PEDs). Our objective was to review (1) bronchodilator use, preference for, and rationale for type of bronchodilator and (2) steroid use and comfort with its use. Methods: Using a modified Dillman method, a piloted, self-administered, 15-question Web-based survey was sent to physicians in the Pediatric Emergency Research Canada database. The database includes physicians working at all 15 Canadian PEDs. Results: A total of $193(71 \%)$ of the 271 surveyed responded. Most respondents (77\%) spent the majority of their clinical time in the PED. The majority $(127 ; 66 \%)$ reported "typically" treating infants with bronchodilators, with respondents evenly split between salbutamol and epinephrine. Most respondents (77\%) using salbutamol report choosing it as they can prescribe it at discharge. Most respondents $(80 \%)$ using epinephrine report choosing it as they believe the medical literature supports its benefit over salbutamol. Few respondents $(5 ; 2.6 \%)$ report "always" using steroids to manage bronchiolitis. The majority of respondents $(120 ; 62 \%)$ report "sometimes" using steroids, with the most common factor influencing the steroid use being illness severity ( $73 \%$ report as an influence). Of those sometimes using steroids, most $(78 \%)$ use only in combination 
with epinephrine. Among those who "never" use steroids, $24 \%$ are concerned about side effects, $30 \%$ are uncomfortable with doses that may be beneficial, and $88 \%$ await further evidence. Most respondents (74\%) report that if steroids were beneficial, they would be most comfortable with a single ED dose, with only $26 \%$ reporting comfort with a longer steroid course. Conclusion: Canadian PED physicians report widespread bronchodilator use in managing bronchiolitis. Use of steroids is less common and strongly influenced by illness severity. There is discomfort among clinicians in using steroids, particularly longer courses, and a preference for a single ED dose.

OP36 Assessment of adverse events in a primary care paramedic ST elevation myocardial infarction bypass program in a large rural area

M. Froats, MD, A. Reed, MD, R. Dionne, MD, CCFP(EM), J. Maloney, MD, FRCP, CCFP(EM), R. Burns, BHSc, I.G. Stiell, MD, MSc, FRCPC; Regional Paramedic Program for Eastern Ontario

Keywords: BLS, bypass, emergency medicine, STEMI

Introduction: In our region, BLS providers bypass closer emergency departments to deliver STEMI patients to PCI centres up to 60 minutes away, exceeding the 30-minute limit recommended by the AHA. The safety of this transport time and non-ALS providers attending STEMI patients during bypass has been questioned. This study was conducted to determine the frequency and nature of adverse events in a BLS STEMI bypass program in a large rural area. Methods: We conducted a health records review for patients transported by a rural BLS EMS agency under bypass to our regional PCI centre. Patients were eligible if they had less than 12 hours of chest pain, a STEMI-positive ECG, and a drive time to the PCI centre within 60 minutes. We determined transport times and adverse events during transport, which were bradycardia $(<50 \mathrm{bpm})$, tachycardia $(>140 \mathrm{bpm})$, hypotension (SBP $<$ $90 \mathrm{~mm} \mathrm{Hg}$ ), cardiac arrest, and death. We conducted descriptive data analyses with 95\% confidence intervals. Results: Forty-five consecutive cases were identified between February 2005 and February 2012 The mean age was 61.2 years, with $32(71.1 \%)$ males. The mean transport time was 30.0 minutes, and 13 cases $(28.9 \%)$ exceeded this (range 31-62 minutes). Twenty cases (44.4\%) had an adverse event. The event rate for cases with drive time under 30 minutes was $40.0 \%$ (95\% CI 22.5-57.5), compared to $46.2 \%$ (95\% CI 19.1-73.3) for cases with longer drive times. Of three events occurring after 30 minutes of transport, there was one ventricular fibrillation arrest that responded to one shock and two transient episodes of hypotension. Fifteen $(75.0 \%)$ of the adverse events were transiently abnormal vital signs requiring no intervention. Four adverse events $(20.0 \%)$ would have benefited from an ALS intervention. None of these would have been transported if the vital signs, adverse events parameters, and abnormal GCS were contraindications to bypass. There were no deaths. Conclusion: BLS EMS providers in a rural county may be able to safely bypass the closest ED and transport STEMI patients to a PCI facility provided that there are no vital signs contraindications at the initial presentation. There was no difference in adverse event rate with extended drive times.

\section{Oral Presentations (Lightning Orals)}

LOP01 Prospective syncope study: outcomes in Canadian ED syncope patients

V. Thiruganasambandamoorthy, CCFP(EM), MSc, A. Vaidyanathan, MBBS, L.A. Calder, MD, MSc, FRCPC, P. Ladouceur, RN, G.A. Wells, PhD, I.G. Stiell, MD, MSc, FRCPC; University of Ottawa, Department of Emergency Medicine
Keywords: emergency medicine, management, outcomes, syncope Introduction: Syncope is a common ED complaint, and the extent of the problem in Canada is not known. The aim of this study was to evaluate the management of Canadian ED syncope patients and describe the occurrence of adverse outcomes. Methods: We conducted a prospective cohort study at two academic EDs over 16 months. We included adults with syncope and excluded those with mental status changes, obvious seizure, alcohol/illicit drug use, and head and severe trauma. We collected patient characteristics, ED management, and short-term (30-day) and long-term (31 days to 1 year) adverse outcomes. Adverse outcomes include MI, pulmonary embolism, subarachnoid and significant hemorrhage, and procedural interventions for short term and death, arrhythmia, and structural heart disease for both short and long terms. We also collected treating physicians' prediction probability for short-term outcomes. We assessed for outcomes by computerized tracking of patient records and telephone follow-up. All adverse outcomes were confirmed by two blinded ED physicians. We performed descriptive analyses and a receiver operating characteristic (ROC) curve for prediction probability with 95\% CIs. Results: Of 199,975 ED visits during the study period, 1,615 (0.8\%) visits were due to syncope. We included 1,253 patients ( $77.6 \%$ recruitment) with these characteristics: mean age 54.1 years, females $57.5 \%$, past atrial fibrillation $7.0 \%$, and history of congestive heart failure $3.1 \%$. ED investigations included blood tests $86.1 \%$, ECG $91.8 \%$, and CT head $21.7 \%$. A minority $(15.3 \%)$ had specialist consultation in the ED, and $8.1 \%$ were admitted. Nearly one-quarter $(21.6 \%)$ suffered minor injuries and $1.9 \%$ suffered fractures. Overall, $98(7.8 \%)$ patients suffered adverse outcomes, $69(5.5 \%)$ in the short term: death $0.2 \%$, arrhythmia $2.4 \%$, hemorrhage $0.9 \%$, MI $0.4 \%$, pulmonary embolism $0.2 \%$. Twenty-six patients $(2.1 \%)$ suffered outcomes after ED disposition. An additional $28(2.2 \%)$ patients suffered long-term adverse outcomes: death $0.4 \%$, arrhythmia $1.0 \%$, structural heart disease $0.3 \%$, and cardiac procedures $0.5 \%$. The ROC curve for physician judgment for 30-day outcomes was 0.80 (95\% CI 0.74, 0.86). Conclusion: In this first Canadian prospective syncope study, we found that a small but significant number of patients suffered adverse outcomes after ED disposition and in the long term. There is a need for accurate riskstratification for short-term outcomes and appropriate follow-up for those at risk of long-term adverse outcomes.

LOP02 A national faculty development needs assessment for Canadian academic emergency medicine

G.M. Brown, MD, E. Lang, MD, K. Patel, MD, B. Chung, MD, S. Dong, D. Blouin, MD, J. Sherbino, MD, MEd, C. Hicks, MD, MEd, FRCPC, G. Bandiera, MD, C. Meyers, MD, P. Yoon, MD; University of Calgary

Keywords: academic centre, emergency medicine, faculty development, survey

Introduction: Academic emergency physicians (AEPs) are expected to fulfill ever-expanding roles of leaders, scholars, researchers, and educators. The skills and knowledge that enable success in these roles is supported by faculty development (FD), for which our knowledge of needs is limited. We conducted a national study of Canadian AEPs to determine (1) their current FD participation, (2) a detailed understanding of their FD needs and interests, (3) the perceived barriers to and facilitators for engaging in FD, and (4) the preferred methods of delivery for FD activities. Methods: This was a multicentre, cross-sectional survey of the current FD needs and interests of AEPs in eight academic centres across Canada. The key domains of this piloted instrument were research, social accountability, scholarship, leadership, and education. Multiple-response and 5point Likert scale questions were utilized; frequencies and mean Likert responses are reported. AEPs from each centre were approached 
using a modified Dillman technique of multiple respondent contacts The survey was administered with surveymonkey.com and championed by local faculty. Results: Across eight centres nationwide, 944 AEPs were surveyed, with a response rate of 36\% (range 23-60\%). Most AEPs reported current participation in educational (99\%), leadership (80\%), and scholarly (77\%) FD activities. Although interest in future educational FD activities remains high (87\%), proportionately more interest is expressed for activities that strengthen leadership $(80 \%)$ and scholarly (79\%) skill sets; interest in FD activities to enhance research remains lower $(62 \%)$. Participation in FD is motivated by personal satisfaction (4.4/5) and a desire to enhance bedside teaching (4.1/5). Optimal FD activities are held during weekday lunches $(68.7 \%)$ and located within the hospital of practice $(94.7 \%)$ Conclusion: This study delineates national faculty development interests across a broad range of academic pursuits and provides additional insight as to where faculty development resource allocation and programming efforts should be focused.

\section{LOP03 Evaluation of Ontario's Pay-for-Results Program on emergency department waiting times}

A.S. Boozary, HBMSc, MPP, M. Vermeulen, MSc, A. Guttman, MDCM, MSc, T. Stukel, PhD, M. Schull, MD, MSc; University of Ottawa

Keywords: emergency medicine, pay for performance, waiting times Introduction: In 2008, Ontario launched the Emergency Department (ED) Wait Times Strategy to address overcrowding. One intervention, Pay-for-Results (P4R), provided financial incentives to hospitals to reduce ED length of stay (LOS). We sought to evaluate the effect of P4R on ED LOS. Methods: P4R and comparator sites were compared for the first 12 months after P4R implementation in each of the first 3 years using health administrative data (2008/2009 [P4R-FY1], 2009/2010 [P4R-FY2], and 2010/2011 [P4R-FY3]). ED wait times in the first year of $\mathrm{P} 4 \mathrm{R}$ were compared with baseline (1 year prior); all models controlled for important patient and contextual factors. The primary outcomes were 90th percentile ED LOS, median ED wait time, and time to physician initial assessment (PIA). Other quality of care measures were also compared as potential unintended consequences. Results: There were 19 EDs in P4R-FY1 (with 21 comparator sites); an additional 20 and 22 sites joined in P4R-FY2 and P4R-FY3 (with 34 and 18 comparator sites), respectively. P4R-FY1 sites had the worst baseline performance, highest admission rates, and the most teaching hospitals. In multivariable models, P4R-FY1 was independently associated with small but significant reductions in the median and 90th percentile ED LOS (aOR - 0.14 [95\% CI $-0.19,-0.01]$ and aOR -0.74 $[95 \% \mathrm{CI}-0.98,-0.49]$, respectively) and PIA times (aOR $-0.13[95 \%$ $\mathrm{CI}-0.15,-0.11]$ and aOR -0.24 [95\% CI $-0.29,-0.18]$, respectively) compared to comparator hospitals; P4R-FY2 and P4R-FY3 were also associated with significant reductions in the same outcomes, with the exception of 90th percentile ED LOS, where we found only weak trends toward reduction. Quality of care outcomes showed no significant differences. Conclusion: P4R was associated with small but significant reductions in most ED LOS and PIA measures; baseline performance was inversely associated with improvement. Further research is needed on how best to design and implement such incentives to maximize their effectiveness.

LOP04 Influence of triage time on patient care in children presenting with suspected appendicitis to Canadian pediatric emergency departments

M. Willimann, MD, G.C. Thompson, MD; Alberta Children's Hospital

Keywords: appendicitis, emergency medicine, pediatrics
Introduction: The objective of this study is to determine if time of presentation influences diagnostic and management strategies, health care flow metrics, and patient outcomes in children presenting to the emergency department (ED) for suspected appendicitis. Methods: A secondary analysis of the Pediatric Emergency Research Canada (PERC) appendicitis database collected retrospectively across 11 Canadian pediatric EDs. Children aged 3 to 17 years admitted to hospital with an ED diagnosis of appendicitis or suspected appendicitis were eligible. Subgroup analysis was performed based on time of presentation defined as day $=08: 00-16: 59$; evening $=17: 00-23: 59$; night $=$ 00:00-07:59. Results: A total of 565 children were included (day $=$ 298 [52.7\%]; evening $=183[32.4 \%]$; night $=84$ [14.9\%]). Demographics and severity of illness were similar across triage-time categories. Investigations: Abdominal radiographs were used less often during the day than evening $(\mathrm{OR}=0.58,95 \%$ CI $0.37-0.91)$ and night $(\mathrm{OR}=0.45,95 \%$ CI $0.26-0.78)$. Ultrasonography (US) was more frequently used during the day compared to evening (OR 1.80, 95\% CI $1.21-2.66$ ) but not night (OR 1.11, 95\% CI 0.65-1.90). Management: Day presentations resulted in disposition directly to the operating room (v. ward) more than evenings $(\mathrm{OR}=2.16,95 \% \mathrm{CI}=1.49-3.15)$ but not nights. Use of analgesics, antibiotics, and surgical methodology (open v. laparoscopy) did not differ. Flow Metrics: Day presentations had significantly shorter times from triage to US (305 v. 479 v. 424 minutes; $p<0.001$ ), antibiotic administration (369 v. 437 v. 440; $p=0.03$ ), ED discharge (431 v. 482 v. 503; $p=0.05)$, and surgical intervention (632 v. 864 v. 753; $p<0.001)$. Patient Outcomes: Rates of negative appendicitis and perforation were not different, although patient numbers for these outcomes were small. Conclusion: Children presenting to the ED with suspected appendicitis experience significant differences in care based on time of presentation, particularly related to investigation strategies and hospital flow metrics. A future prospective nationwide study to further define the impact on patient outcomes and health care economics is planned.

LOP05 Follow-up and shared care following discharge from the emergency department for exacerbations of chronic disease M. Schull, MD, MSc, M. Vermeulen, MSc, T. Stukel, PhD, E. Fisher, MD, MPH; Sunnybrook and Women's Hospital

Keywords: chronic disease management, emergency medicine, shared care

Introduction: Ensuring follow-up and care integration between providers in chronic disease (CD) is a challenge facing Canadian and many other health systems. One example is care provided to CD patients following discharge from the emergency department (ED). Little is known about the comprehensiveness of follow-up following ED visits. Our objective was to describe the follow-up of patients after ED discharge for an exacerbation of three common and serious CDs. Methods: Using health administrative databases, we conducted a retrospective, observational study of Ontario ED visits (2005-2008). We identified patients who had an ED visit due to an exacerbation of congestive heart failure (CHF), diabetes, or chronic obstructive pulmonary disease (COPD) that resulted in discharge home. For each patient, we determined whether they were seen by a primary care physician, a specialist related to their $\mathrm{CD}$, both (defined as shared care), or neither during a 30-day follow-up period. Results: We identified 15,044 CHF, 32,148 diabetes, and 31,910 COPD ED visits resulting in discharge from $73 \mathrm{EDs}$; mean (SD) age was 75.7 years (11.84), 72.3 years $(10.4)$, and 63.9 years (15.9), respectively. Among CHF patients, $14.9 \%$ saw no physician, $65.3 \%$ saw only a primary care physician, $5.3 \%$ saw a specialist only, and $14.4 \%$ received shared care in the 30 -day followup period. Among diabetic patients, $26.7 \%, 66.4 \%, 2.7 \%$, and $4.1 \%$ saw no physician, a primary physician only, or a specialist only and received shared care, respectively. Among COPD patients, 31.8\% saw 
no physician, $62.5 \%$ saw only a primary care physician, $2.0 \%$ saw a specialist only, and 3.7\% received shared care. Conclusion: Between 15 and $31 \%$ of patients saw no physician in the 30 days following an ED visit for an exacerbation of their CD. Most follow-up is with a family doctor alone. Shared care involving a family doctor and specialist is infrequent. ED-based interventions could help improve follow-up rates, which in turn could improve patient outcomes.

LOP06 Evaluation of an emergency medical services treat-andrelease protocol for out-of-hospital patients presenting with supraventricular tachycardia

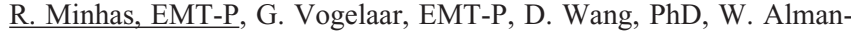
soori, MSc, E. Lang, MD, I. Blanchard, EMT-P, MSc, G. Lazarenko, MD, A. McRae, MD; Alberta Health Services EMS

Keywords: emergency medicine, EMS, supraventricular tachycardia Introduction: Many patients with paroxysmal supraventricular tachycardia (SVT) present to emergency medical services (EMS). Most EMS agencies have protocols to allow advanced care paramedics (ACPs) to treat SVT using nodal blocking agents. This study evaluated the safety and effectiveness of a treat-and-release $(\mathrm{T}+\mathrm{R})$ protocol to allow ACPs to treat SVT in the field, without transport to an emergency department (ED). Methods: This study linked data from the Alberta Health Services (AHS) EMS Electronic Patient Care Record (EPCR) database for the City of Calgary to the AHS Calgary Zone Regional Emergency Department Information System (REDIS) database. All SVT patients in the EPCR database treated between September 1, 2010, and September 30, 2012, were identified and linked to the REDIS database. EPCR reviews were completed on all patients who were $\mathrm{T}+\mathrm{R}$. The EPCR and REDIS databases were queried to identify any $\mathrm{T}+\mathrm{R}$ patient re-presenting to EMS or an ED within 72 hours of initial treatment. Results: There were 75 SVT $\mathrm{T}+\mathrm{R}$ patient encounters. All criteria for the $\mathrm{T}+\mathrm{R}$ protocol were met and documented for $43 / 75$ patients $(57 \%)$. Due to a limitation of the EPCR platform, some criteria were not documented on early study PCR forms. With incomplete records excluded, 54 of 60 patients $(90 \%)$ met $\mathrm{T}+\mathrm{R}$ protocol criteria. The most common protocol breaches were failure to provide patients with printed instructions on when to call $911(n=14)$ and failing to ensure that a responsible adult was with the patient for 4 hours posttreatment $(n=5)$. Forty patients received vagal manoeuvres; however, this was rarely successful $(5 / 40,12.5 \%)$. Sixty-seven of 75 patients $(89 \%)$ were treated with adenosine; all patients were successfully converted with a single dose of either $6 \mathrm{mg}(n=8)$ or $12 \mathrm{mg}(n=59)$. The remaining patients converted to sinus rhythm spontaneously. Ten $\mathrm{T}+\mathrm{R}$ patient encounters led to EMS re-presentation within 72 hours. All 10 re-presentations were by the same patient. Four $\mathrm{T}+\mathrm{R}$ patient encounters led to an $\mathrm{ED}$ presentation within 72 hours. All of these were again by the same patient. Two ED presentations led to treatment and discharge for SVT, while two resulted in admission for conditions unrelated to SVT. Conclusion: EMS practitioners are able to follow a $\mathrm{T}+\mathrm{R}$ protocol with reasonable adherence to protocol requirements. This protocol appears to be effective and safe in selecting appropriate patients for $\mathrm{T}+\mathrm{R}$. A $\mathrm{T}+\mathrm{R}$ protocol appears to be a viable option for patients presenting with SVT in the prehospital setting.

\section{LOP07 Hypertension in the ED: are blood pressure devices} related to increased $\mathrm{ED}$ visits?

A.Y. Wong, MD, A. Zia, MD, C. Atzema, MD, MSc; University of Toronto

Keywords: blood pressure measurement, emergency medicine, home blood pressure measurement, hypertension
Introduction: While hypertension is a common reason to present to an emergency department (ED), no consensus exists on ED management in the absence of end-organ failure. We sought to characterize these patients, to quantify the proportion of visits secondary to a pharmacy or home blood pressure reading, and to describe their ED course and disposition. Methods: In this chart review at two sites, we performed chart abstraction on patient visits with a final diagnosis of hypertension that were made between April 2009 and March 2010. We used descriptive statistics to describe patient characteristics, reason for visit, ED management, and disposition. Results: Among 545 qualifying patients, the mean age was 64.2 , and $59.9 \%$ were female. Most had a prior diagnosis of hypertension $(77 \%)$. The proportion of patients who presented to the ED after a reading at home, a pharmacy, or via MD referral was $48.8 \%, 8.8 \%$, and $11.6 \%$, respectively; $40.7 \%$ (95\% CI 36.6 to $45 \%)$ received ED antihypertensive therapy, $10.5 \%$ (95\% CI 8.0 to $13.3 \%$ ) were admitted, and $55.8 \%$ ( $95 \%$ CI -51.5 to $60 \%$ ) discharged patients received an outpatient antihypertensive prescription. Among patients who utilized a self-measurement device (at home or pharmacy) prior to presentation, 59\% were asymptomatic; 30\% (95\% CI - 24.9 to 35.4) of patients received ED antihypertensive treatment, $3.9 \%$ (95\% CI - 2 to 6.7$)$ were admitted, and $60.3 \%$ (95\% CI - 54.6 to 65.8) of discharged patients were given an antihypertensive prescription. Conclusion: The majority of patients presented secondary to elevated readings on self-measurement devices. Almost all of these patients $(>96 \%)$ were discharged home from the $\mathrm{ED}$, the majority of whom received no ED intervention. Patient education around when to seek emergency care and how to manage elevated blood pressure values might decrease unnecessary ED visits in this patient group.

LOP08 The safety of prehospital continuous positive airway pressure use by primary care paramedics

S. Cheskes, MD, CCFP(EM), FCFP, L. Turner, PhD, S. Thomson, ACP, N. Aljerian, MD; Rescu, Li Ka Shing Knowledge Institute, St. Michael's Hospital

Keywords: continuous positive airway pressure, emergency medical services, emergency medicine, paramedic

Introduction: Previous studies have demonstrated the feasibility of continuous positive airway pressure (CPAP) use by primary care paramedics (PCPs). We sought to study the safety of prehospital CPAP when used by PCPs compared with advanced care paramedics (ACPs). Methods: Data were abstracted from ambulance call reports and hospital records for a 1-year period beginning June 25, 2009. Inclusion criteria were based on the provincial medical directive for CPAP use, hospital admission with known outcome, and no DNR documentation on the patient chart. We defined safe prehospital use of CPAP as comparable rates of in-hospital mortality, intubation, scene time, and confirmed in-hospital diagnosis of pulmonary edema/CHF or COPD for patients treated by PCPs versus ACPs. Results: Of 231 consecutive cases of CPAP, 78 met the study criteria, 20 treated by PCPs and 58 by ACPs. Whether treated by ACPs or PCPs, no significant difference was found for rates of in-hospital intubation (11/58 or 19\% for ACP and $3 / 20$ or $15 \%$ for PCP, $p=.69$ ), mean (SD) scene time (minutes) (17.6 [4.9] and 16.6 [5.2] [ $p=.41]$ for ACPs and PCPs, respectively), or the proportion of patients with an in-hospital confirmed diagnosis of COPD, CHF, or pulmonary edema $(75.9 \%$ and $75 \%$ for ACP v. PCPtreated patients $[p=.94])$. No significant association between mortality and paramedic level was noted $(3 / 58$ or $5.2 \%$ for ACP and $3 / 20$ or $15 \%$ for PCP, $p=.16$ ). Conclusion: Although our sample size was small, we observed no significant difference in rates of in-hospital intubation, mortality, scene time, or diagnostic accuracy in patients treated with prehospital CPAP by PCPs when compared to ACPs. Based on our definition of safety, it appears that prehospital CPAP can be safely used by paramedics trained to the PCP level. 
LOP09 Lactate clearance for guiding the management of septic patients in the emergency department: a systematic review I. Sudbury, MD, A.S. Khory, MD, E. Lang, MD, A. McRae, MD; University of Calgary

Keywords: emergency medicine, lactate clearance, sepsis

Introduction: Lactate clearance has been proposed as a guide to the management of sepsis since it has been previously demonstrated to correlate with in-hospital mortality and improve outcomes when added to a standard sepsis protocol. By potentially avoiding the need for early central venous sampling, lactate clearance can remove a major barrier to early goal-directed therapy-based protocols in busy urban emergency departments as well as in community and rural hospitals. We conducted a systematic review of the literature to define the prognostic significance of lactate clearance in predicting mortality. Methods: A literature search was performed using Medline, Medline in progress, Cochrane Database of Systematic Reviews and Register of Controlled Trials, PubMed, and EMBASE, as well as all relevant conference abstracts since 2007 for studies of prospective cohort study design or higher. Relevant abstracts were screened by two independent reviewers, and any discrepancies were settled by adjudication by two additional reviewers. Lactate clearance was defined by a reduction in serum lactate level by $>10 \%$ over a 2 - to 6 -hour period. GRADE criteria and the Newcastle-Ottawa scale were used to assess the quality of included studies. Results: A total of 669 original articles were screened and five high-quality studies were selected, in consensus between reviewers based on predetermined inclusion and exclusion criteria. Of the five studies, three examined mortality based on a lactate clearance value of greater or less than $10 \%$, and unpublished mortality data were obtained from a fourth study. Due to the heterogeneity of the five studies selected, meta-analysis was not performed. A lactate clearance of greater than $10 \%$ showed a survival advantage in all studies. Conclusion: Lactate clearance is a predictor of mortality for septic patients presenting to the emergency department and is a reliable and less invasive method of monitoring response to therapy. There is potential for lactate clearance to replace a central venous oxygen saturation goal in sepsis protocols.

LOP10 Effect of emergency department crowding on ED disposition for transient ischemic attack and minor stroke patients

M. Ben-Yakov, MDCM, M. Kapral, MD, MSc, FRCPC, J. Fang, PhD, S. Li, PhD, M. Schull, MD, MSc; Institute for Clinical Evaluative Sciences

Keywords: emergency medicine, overcrowding, stroke, TIA Introduction: TIA and stroke are leading causes of disability and carry important risk of subsequent morbidity. Assessment and risk factor modification can occur in hospital for high-risk patients or in specialized outpatient clinics for lower-risk patients. Current risk stratification tools are unreliable, and context factors such as the degree of emergency department crowding may influence the decision to admit or discharge a TIA or minor stroke patient. We sought to determine the association between ED overcrowding and the likelihood of hospitalization for ED patients with TIA or minor stroke (defined as a Canadian Neurological Score $>8$ ). Methods: A retrospective cohort study from the Registry of the Canadian Stroke Network (12 Ontario EDs from 2003 to 2008) linked to administrative databases. Descriptive statistics and patient-level multivariate logistic regression were used. The main outcome was ED disposition (discharge v. admission to hospital). We adjusted for important patient, ED shift, and hospital characteristics. The main exposure was degree of ED crowding, measured as mean hours of ED length of stay (ED LOS) for patients in the same ED, shift, and CTAS categories. Results: A total of 9,759 patients with TIA (47.2\%) and minor stroke
(52.8\%) were included. The discharge rate from ED was $25.5 \%$ for stroke and $74.5 \%$ for TIA patients. Overall, increasing levels of crowding were associated with reduced risk of discharge. However, when stratified by ED annual volumes, increasing crowding was associated with statistically significant increased risk of ED discharge in higher-volume sites, while the opposite effect was seen in lower-volume sites. Among high-volume EDs, the likelihood of discharge for TIA/stroke patients increased steadily with increasing ED crowding, from an aOR $=1.56(95 \%$ CI $0.87-2.81)$ with low crowding (mean ED LOS of 3-4 hours) to an aOR $=3.72$ (95\% CI 2.11-6.55) with high crowding (mean ED LOS $>7$ hours), compared to no crowding (mean ED LOS $<3$ hours). Conclusion: ED crowding is associated with increased risk of discharge of TIA and minor stroke patients, but only in higher-volume settings. This may reflect the burden of ED crowding in higher-volume sites and/or better access to rapid outpatient specialized follow-up. Given the lack of validated risk stratification tools, ensuring appropriate disposition among high-risk patient populations, even during crowded conditions, seems paramount.

LOP 11 Does prehospital nitroglycerin for chest pain cause hypotension in acute inferior wall STEMI? A retrospective cohort study

L. Robichaud, MD, D. Ross, MD, M. Proulx, PCP MSc, S. Légaré, PCP, C. Vacon, EMT-B PhD, X. Xue, MSc, E. Segal; McGill Emergency Medicine Residency Training Program

\section{Keywords: emergency medicine, EMS, STEMI}

Introduction: Patients with inferior ST elevation myocardial infarction (STEMI), associated with right ventricular infarction, are potentially at higher risk of developing hypotension when administered nitroglycerin (NTG). However, current basic life support primary care paramedic (PCP) protocols do not differentiate location of STEMI prior to NTG administration. Methods: We sought to determine if NTG administration is more likely to cause hypotension (systolic blood pressure $<90 \mathrm{~mm} \mathrm{Hg}$ ) in inferior STEMI compared to noninferior STEMI. We conducted a retrospective chart review of prehospital patients with chest pain of suspected cardiac origin and computerinterpreted prehospital ECGs indicating "ACUTE MI." We included all local STEMI cases identified as part of a provincial STEMI registry project. Univariate analysis was used to compare differences in proportions of hypotension after nitroglycerin administration, drop in systolic blood pressure $\geq 30 \mathrm{~mm} \mathrm{Hg}$, and hypotension on initial prehospital blood pressure between patients with inferior wall STEMI and those with STEMI in another region (noninferior). Results: Over a 29-month period, we identified 1,466 STEMI cases. Of those, 798 (54.4\%) had complete data and received NTG. Hypotension occurred post NTG in 36/461 inferior STEMIs and 29/337 noninferior STEMIs, $7.8 \%$ versus $8.6 \%, p=0.69$. A drop in systolic blood pressure $\geq$ $30 \mathrm{~mm} \mathrm{Hg}$ occurred in $23.5 \%$ of inferior STEMIs and $23.8 \%$ of noninferior STEMIs, $p=0.91$. Initial hypotension was noted in significantly more inferior STEMIs compared to noninferior STEMIs, 9.9\% versus $4.9 \%, p=0.005$. Interrater agreement for chart review of the primary outcome was excellent $(k a p p a=0.94)$. Conclusion: Patients with chest pain and inferior wall STEMI on their computer-interpreted prehospital ECG who receive nitroglycerin do not seem to develop hypotension more frequently than patients with STEMI in other territories. Computer-interpreted prehospital ECGs cannot be used as the sole predictor for patients who may be at higher risk for hypotension following NTG administration.

LOP12 Cardiac arrest post-return of spontaneous circulation emergency medical services initiation of therapeutic hypothermia: chillingly low rates and contributing factors 
R.D. Crane, MD, A.O. Arthur, PharmD, S.H. Thomas, MD, MPH, J.M. Goodloe, MD; The University of Oklahoma School of Community Medicine

Keywords: cardiac arrest, emergency medicine, EMS, therapeutic hypothermia

Introduction: Out-of-hospital cardiac arrest (CA) is historically associated with low neurologically intact survival rates. Multiple studies conclude that inducing therapeutic hypothermia (TH) within a few hours post-return of spontaneous circulation (ROSC) improves neurologically intact survival rates. Many emergency medical services (EMS) systems initiate TH to facilitate timely hypothermia postROSC. The study system serves two major metropolitan areas in a southwestern US state and has post-ROSC TH standing orders. The study's purpose was to identify frequency of TH initiation and reason(s) contributing to lack of TH initiation in a CA patient cohort. Methods: Retrospective chart review for CA occurring January 5, 2010 (initiation date of standing order TH care in the study system), to January 5, 2011. The 356-patient cohort was defined by paramedic clinical assessment of CA and subsequent occurrence of ROSC. All patients were transported by the study system to hospitals capable of TH. A total of 356/356 (100\%) EMS patient care records were examined to determine if TH was initiated by paramedics and, in cases without TH initiation, to determine contributing reasoning and/or barriers. Results: A total of $136 / 356$ (38.2\%) study cohort patients had paramedic-initiated TH. Multivariate logistic regression analysis revealed two contributing factors: (1) CA location in the western major metropolitan area (both areas with the same TH education and equipment) and (2) non-EMS-witnessed CA. Among 220/356 (62.8\%) study cohort patients not receiving paramedic-initiated $\mathrm{TH}, 123(34.5 \%$ overall cohort) did not meet EMS TH treatment protocol inclusion criteria and $44(12.3 \%)$ exhibited one or more EMS TH treatment protocol exclusion criteria. A potential influence was seen in $66(18.6 \%)$ patients without TH who had CA within 2 miles (estimated transport time 5 minutes or less) from the destination hospital. Conclusion: In this study, a minority of CA patients with field ROSC receive TH despite standing orders for such. Reasons are multifactorial, including appropriate application of clinical inclusion/exclusion criteria, distance-time post-ROSC to destination hospital, whether CA nonwitnessed by EMS, and unclear etiologies that require increased thoroughness of patient care report documentation to elucidate.

LOP13 A nationwide survey of emergency medical services standards of care for cardiac arrest: variability among America's largest urban systems

J.M. Goodloe, MD, D. Lehrfeld, MD, R.L. Fowler, MD, T.D. Valenzuela, MD, MPH, P.E. Pepe, MD, MPH, K.S. Schrank, MD; The University of Oklahoma School of Community Medicine

Keywords: cardiac arrest, emergency medicine, EMS, resuscitation Introduction: Prehospital resuscitation care for cardiac arrest (CA) impacts morbidity and mortality. Variability in EMS care standards for CA exists among the largest urban EMS systems with the 2010 ILCOR/AHA consensus guidelines. This study's purpose was to describe CA care standards in mid-2011 in the EMS systems in the United States Metropolitan Municipalities EMS Medical Directors Consortium. Methods: The study utilized a standardized Internetbased survey platform conducted with the medical directors for the United States-based systems in the Consortium. Surveys were completed from June 15 to August 15, 2011, with a goal to collect responses in a narrow time interval to minimize the effect of revisions in care standards. Results: Thirty-four EMS systems met inclusion criteria, representing care standards applied to over 50 million Americans, with 34 medical directors responding (100\% survey response).
Among over 60 variables queried, 14 (42\%) initiate Basic Life Support at 30 compressions: 2 ventilations per consensus guidelines. Seven (21\%) specify continuous chest compressions from the start of resuscitation with no pause but interposed ventilations. Nine (26\%) specify chest compressions only during the first 2 to 3 minutes, with either passive oxygenation by oxygen mask $(6 ; 18 \%)$ or no oxygen $(3 ; 9 \%)$. Airway management by primary endotracheal intubation after initial noninvasive care occurs in $12(35 \%)$ systems. Six (18\%) primarily utilize supraglottic devices, with 14 (41\%) allowing paramedics to decide between endotracheal and supraglottic device placement. Thirty $(88 \%)$ systems utilize continuous waveform capnography. Initial approach to non-EMS-witnessed ventricular fibrillation (VF) is chest compression prior to first defibrillation in $30(88 \%)$ systems. Eighteen (52\%) escalate defibrillation energy settings, $4(12 \%)$ utilizing double sequential defibrillation. Twenty (59\%) systems initiate therapeutic hypothermia in the field. Conclusion: Wide variability in CA care standards exists in America's largest urban EMS systems. Although medical directors are uniformly aware of the 2010 ILCOR/AHA consensus guidelines, current practices often provide more continuity of chest compressions than enabled via consensus guidelines. Endotracheal intubation, a past mainstay of CA airway management, is de-emphasized. Immediate defibrillation of non-EMS-witnessed VF is rare.

LOP14 Angioscan combined with V/Q spect to investigate pulmonary embolism is associated with a lower mortality

J. Martel, R3, M. Nadeau, R3, R. Daoust, MD, J. Paquet, Statistician, J. Chauny, MD, MSc; Université de Montréal

Keywords: double investigation, emergency medicine, mortality, pulmonary embolism

Introduction: A significant proportion of the patients have a double radiologic investigation (V/Q SPECT and Angioscan) to diagnose pulmonary embolism (PE). This incurs higher costs, higher irradiation dose, and possibly longer length of stay (LOS). We studied the impact of this double investigation (DI) on mortality. Methods: We conducted a post hoc analysis of prospectively archived data that included patients who underwent an investigation for a PE (V/Q SPECT, Angioscan, and DI) at the ED of Hôpital du Sacré-Coeur de Montréal from April 2008 to October 2012. Patients were selected from a computerized database of medical prescriptions (MediaMed Technologies). Patient characteristics, radiology reports, and diagnosis were then extracted from our database, and mortality was obtained from the Québec provincial mortality registry. We performed descriptive, oneway ANOVA and chi-square statistics. Results: During our study period, 3,913 patients were investigated for PE. Their mean age was 61.3 years $(\mathrm{SD} \pm 19.6)$, and $50.3 \%$ were men, $2,568(65.6 \%)$ had Angioscan, 1,220 (31.2\%) had V/Q SPECT, and 125 (3.2\%) had both investigations. These three groups had similar characteristics, except for the Angioscan group that was composed of more men (59\% v. 34\% for the other groups), younger patients (59.7 v. 64 years old), with less comorbidities (past PE, diabetes, high blood pressure, and coronary artery disease); however, they were hospitalized more often $(68 \% \mathrm{v}$. V/Q SPECT $41 \% \mathrm{v}$. DI $44 \%)(p<0.001)$. LOS was 41.4 hours for DI compared to 31.4 hours for Angioscan and 30.2 for V/Q SPECT ( $p<$ 0.001 ). During our follow-up period (2.3 years, $\mathrm{SD} \pm 1.3$ ), the DI had a lower mortality $(8.8 \%)$ than the Angioscan $(22.2 \%)$ and V/Q SPECT $(14.5 \%)(p<0.001)$. Conclusion: The higher costs, higher irradiation dose, and longer LOS related with the DI for investigating PE appear justified by its association with a lower mortality. More research is needed to identify the optimal investigation strategy for PE.

$L O P 15$ Acute migraine treatment in the emergency setting: a systematic review and mixed treatment comparison 
B.H. Rowe, MD, MSc, CCFP(EM), D.M. Dryden, PhD, E. SumamoSchellenberg, MPH, B. Vandermeer, MSc, B. Friedman, MD, MSc, I. Colman, PhD; University of Alberta

Keywords: emergency medicine, migraine, systematic review, therapy Introduction: A variety of agents are potentially beneficial as treatments for acute migraine headaches; however, controversy exists regarding their relative effectiveness. This systematic review was designed to compare the effectiveness of parenteral interventions to treat migraine headaches in adults presenting to emergency departments. Methods: We searched 10 databases to January 2012, conference proceedings, clinical trials registers, and reference lists. Two reviewers independently selected studies, assessed methodological quality, and graded the evidence. One reviewer extracted and a second verified data. Study quality was rated using the Risk of Bias tool. Findings were qualitatively summarized using mean differences (MD) and $95 \%$ confidence intervals $(\mathrm{CI})$, and meta-analyses and a mixed treatment comparison were conducted. Results: Nine classes of drugs were investigated in 71 controlled trials. The risk of bias was low for $28 \%$ of trials, unclear for $61 \%$, and high for $11 \%$. Active interventions were more effective than placebo for pain relief and headache recurrence. Most head-to-head comparisons for pain reduction were based on single trials, resulting in insufficient strength of evidence (SOE). The mixed treatment comparison showed that the most effective treatments for pain reduction were combination therapy (e.g., dihydroergotamine [DHE] added to either neuroleptics or metoclopramide) or neuroleptic monotherapy (low SOE). Metoclopramide monotherapy, opioids, and NSAIDs were the next most effective treatments. Conclusion: Many agents are effective in the treatment of acute migraine headache when compared to placebo. Several treatments provide insufficient evidence for continued use. Neuroleptic monotherapy or combination treatments with two or more active agents appear to be the most effective options for acute pain relief.

LOP16 Epidemiology of patient presentation to the Canadian Medical Assistance Teams (CMAT) field hospital after the 2010 Haiti earthquake

R. Chin, MD, P. San Agustin, MD, C. Tarun, BSc (Candidate), R. Chetram, BSc (Candidate), J.M. Franc, MD, MSc, CCFP(EM), V. Rzepka, BScN, C. Villa-Roel, MD, PhD (Candidate), B.H. Rowe, MD, $\mathrm{MSc}$, CCFP(EM); Universita del Piemonte Orientale

Keywords: disaster, earthquake, emergency medicine, surveillance Introduction: The Canadian Medical Assistance Teams (CMAT) early response to the 2010 Haiti earthquake disaster involved establishing a field hospital to manage medical and surgical pathology. The goals of this study were to describe the patient presentations and their progression at this field hospital and develop strategies to prepare for future disasters. Methods: This is a prospective study of patient encounters in Haiti immediately following the 2010 Haiti earthquake. Data for the study were collected by nurses or paramedics using a one-page log at the triage and maintained from day 6 to 43 days after the earthquake. The study codebook and database were based on international disaster publications and study team consensus; paper-based forms were entered into an $A C C E S S$ database using double data entry and consensus resolution of disagreement. Data were subgrouped into trauma, wound care, obstetrics/gynecology, and medical/other. Statistical analysis and graphical representation of data were performed to examine differences in presentation among post earthquake days (e.g., 6-12, 13-19, 20-26, $27-33,34-41)$, age, and sex. Results: From day 6 post earthquake until day $43,5,108$ patients were seen at the CMAT field hospital, $67.7 \%$ adult and $32.1 \%$ pediatric. Overall, medical complaints $(70.4 \%)$ outnumbered wound care (14.1\%), obstetrics/gynecology $(7.6 \%)$, and trauma $(7.9 \%)$. Traumatic presentations decreased dramatically from collection day 1 and then reached a plateau after week 1 of the CMAT operation, while the other three categories increased and accounted for the majority of patient presentations. Conclusion: Preparing for and responding to a disaster, which by definition entails limited resources, are complex. This study provides valuable information by characterizing the patient presentations to an acute response hospital after an earthquake. Our findings provide a framework that the disaster medicine community can use for response preparation.

LOP17 Availability of prehospital information at point of care in the ED and the utility of a structured triage nursing extraction tool: a before and after study

E.S. Kwok, MD, MHA, MSc, FRCPC, R. Dionne, MD, CCFP(EM), J. Maloney, MD, FRCP, CCFP(EM), D. Picard-Stencer, RN, BScN, MPA, C. Beland, RN, MScN, ENC(C), C. Poulin, RN, BScN, ENC(C), B. Keleher, BA, I.G. Stiell, MD, MSc, FRCPC; University of Ottawa, Department of Emergency Medicine

Keywords: emergency medicine, EMS, transfer of care, triage Introduction: Accurate details pertaining to EMS initial assessment/treatment of patients arriving at the ED are extremely important for safe and effective transfer of care. However, Ontario regulations allow 48 hours for ambulance call reports (ACRs) completion, often leading to unavailability of critical prehospital information at point of care. The purpose of this study was to examine the incidence and characteristics of missing ACRs at point of care and to evaluate the impact of implementing a new triage nursing tool for extracting prehospital information. Methods: This prospective before/after interventional study took place at a tertiary academic centre over a 6-month period. Study forms were attached to the charts of all patients arriving at the ED via ambulance. The treating ED physicians completed the forms at the point of care. Two months into the study, our ED nursing charts were modified to include a new structured EMS information extraction tool and its rollout facilitated by educational sessions for triage nurses. Impact was measured by a 6-point Likert scale as scored by the treating ED doctors. Physician impressions on how prehospital information affected various aspects of patient care were also graded. Data were entered/analyzed in an Excel database and presented using descriptive statistics. Results: A total of 455 completed study forms were included for analysis (182 before; 273 after). ACRs were available at the point of care in $3.7 \%$ of cases, while the treating physician felt that prehospital information would have been useful in $76.1 \%$ of those cases. The top three potentially useful pieces of information as valued by the treating physician were (1) vitals at the scene; (2) collateral history; and (3) patient's history at the scene. The usefulness of prehospital information captured by our new structured triage extraction tool was improved when comparing before/after rankings on a 6point Likert scale: mean (before) $=3.68(\mathrm{SD} \pm 1.56)$ versus mean $($ after $)=3.96(\mathrm{SD} \pm 1.40)$, with the improvement reaching statistical significance ( $U$ test $p<0.01$ ). ED diagnostic/investigation choices and overall patient safety were the most affected. Conclusion: We observed significant unavailability of ACRs at the point of care in the ED, despite their potential usefulness. The most valuable missing pieces of information as rated by physicians were specific to initial assessments and history gathered by EMS personnel. A new structured triage extraction tool appears to help mitigate loss of critical prehospital information affecting various aspects of patient care.

LOP18 A randomized trial of opinion leader communication to improve primary care follow-up after emergency department discharge for acute asthma and COPD: the TLAL TRIAL

B.H. Rowe, MD, MSc, CCFP(EM), C. Villa-Roel, MD, PhD (Candidate), L. Rodriguez, MD, PhD (Candidate), E. Cross, MD (Candidate), 
S. Couperthwaite, BSc, J. Victor, BScN, M. Bhutani, MD, FRCPC University of Alberta

Keywords: asthma, COPD, emergency medicine, follow-up Introduction: Asthma and chronic obstructive pulmonary disease (COPD) are the most common chronic wheezing conditions that present as exacerbations to the emergency department (ED). Following evidence-based discharge treatment, close follow-up and review with primary care providers (PCP) are part of guideline recommendations. The objective of this study was to compare opinion leader (OL)-specific communication with standard care to increase PCP contact and reduce relapses after ED discharge. Methods: A block-randomized controlled clinical trial was conducted at one $\mathrm{ED}$ in Edmonton, $\mathrm{AB}$, using concealment of allocation and multiple levels of blinding. The physicians of patients with MD-diagnosed asthma or COPD exacerbations were randomized at discharge to receive a standard faxed ED chart or a personalized Lung Attack Alert letter signed by a local OL. All patients received evidence-based discharge medications (asthma: prednisone and inhaled corticosteroids; COPD: prednisone and antibiotics). Patients were followed at 30 and 90 days, and outcomes were electrically verified. Descriptive data are reported using proportions and medians with interquartile range (IQR), where appropriate, and compared using chi-square and Mann-Whitney tests. Time to event is reported using log-rank tests. Results: From 786 screened patients, 136 were consented and 128 were fully enrolled. Overall, 65 received intervention (41 asthma; 24 COPD) and 63 control (39 asthma; 24 COPD) interventions; $53 \%$ were women, and the median age was 46 . Many gaps in management and prevention were identified in both COPD and asthma patients. Follow-up was $84 \%$ at 30 and $71 \%$ at 90 days. At the 90-day contact, PCP follow-up occurred in $36(55 \%)$ intervention and $35(56 \%)$ control patients ( $p=0.37)$; the time to follow-up was similar ( 25 v. 20 days; $p=0.79)$. Intervention (34 [52\%]) and control (28 [44\%]) groups had similar 90-day relapses $(p=0.47)$; the time to relapse was similar ( 40 v. 30 days; $p=0.47$ ). Results were similar for 30-day outcomes. Conclusion: Overall, notification of the PCP using a personalized Lung Attack Alert letter signed by a local OL did not enhance follow-up or reduce relapse compared to standard care for patients with acute asthma and COPD. While follow-up and management review are recommended, most follow-ups occur late and did not address identified gaps. Additional efforts are required to stabilize patients after discharge.

\section{LOP19 Diagnosis and treatment of anaphylaxis in Edmonton-} area emergency departments

L. Lovstrom, BSc, MSc (Candidate), K. Nagra, BSc, MD (Candidate), T. Chung, BSc (Candidate), S. Couperthwaite, BSc, C. Vandenberghe, MSc, C. Villa-Roel, MD, PhD (Candidate), B.H. Rowe, MD, MSc, CCFP(EM); University of Alberta

Keywords: allergy, anaphylaxis, clinical practice guidelines, emergency medicine, epinephrine

Introduction: Anaphylaxis is a life-threatening allergic reaction that often results in presentation to the emergency department (ED); however, management in Canadian EDs has not been extensively examined. This study aimed to identify gaps in anaphylaxis management in six urban EDs and make recommendations to improve patient outcomes in the future. Methods: Using a retrospective chart review approach, research staff reviewed hospital charts at six Edmonton-zone hospitals for patients with at least one visit for anaphylaxis in 2011. Information was collected using a standardized form by trained research staff unaware of the study hypothesis, and anaphylaxis confirmation was based upon current diagnostic guidelines for anaphylaxis. Double data extraction was conducted on the first 20 charts to enhance data collection reliability. Descriptive data are reported using proportions and medians with interquartile range (IQR), where appropriate. Results: A total of 150 charts were reviewed at the six sites; however, only 104 (69\%) were confirmed anaphylaxis cases. Arrival was via ambulance in $39(38 \%)$ cases, and CTAS scores were frequently 1 or 2 (77 [69\%]); $64(62 \%)$ had received pre-ED treatment, including administration of epinephrine (33 [64\%]). The ED treatment included systemic corticosteroids (86 [83\%]); epinephrine (62 [60\%]); intravenous saline (63 [61\%]); and antihistamines (73 [70\%] $\mathrm{H}_{1}$ and $54[52 \%] \mathrm{H}_{2}$ agents). Repeat epinephrine was required infrequently (12 [19\%]). The overall ED length of stay was 3.93 (IQR: 2.85, 6.20) hours, and 96 (92\%) were discharged. At discharge, patients received prescriptions for epinephrine (56 [53.8\%]), of which $82 \%$ were new; oral corticosteroids (56 [53.8\%]); $50 \mathrm{mg}$ prednisone for 3 [IQR: 2, 5] days); and antihistamines (34 [34\%]). Discharge planning included an action plan (38 [37\%]) and referral to an allergy specialist (12 [12\%]); MedicAlert (1 [1\%]) and website (0 [0\%]) information was uncommonly documented. Conclusion: Administrative data overestimate anaphylaxis by approximately $30 \%$ due to MD overcall of allergic reactions. Overall, more aggressive pharmacologic management of anaphylaxis and lower admissions occur for anaphylaxis in this Canadian centre compared to previous US ED data. Attention to anaphylaxis prevention was infrequently documented.

LOP20 Trauma nontechnical (TNT) training: piloting and assessment of a novel, interprofessional, simulation-based nontechnical skills curriculum for trauma resuscitation

A. Doumouras, MD, I. Keshet, MD, N. Ahmed, MD, PhD, FRCS, C. Hicks, MD, MEd, FRCPC; University of Toronto

Keywords: emergency medicine, medical education, patient safety Introduction: Medical error is common in trauma, occurring in up to $100 \%$ of trauma resuscitations. The majority of observed errors are nontechnical in nature, stemming from failures in communication, leadership, situation awareness, and team performance. We describe the piloting and assessment of a novel, interprofessional, simulation-based nontechnical skills curriculum for trauma resuscitation (the TNT curriculum). Methods: Six interprofessional teams of second-year postgraduate trainees and emergency nurses took part in three standardized trauma simulations, which were video-recorded for subsequent analysis. Teams received focused feedback on nontechnical performance from a trained facilitator after each scenario. Our primary outcome was team performance, evaluated by two blinded reviewers using validated behavioural rating scales, the Mayo Team Scale and the Ottawa CRM Global Rating Scale. To assess skill retention, two teams returned after 6 months and participated in three new resuscitation scenario-debriefing pairs. To assess attitudinal shifts toward team-based behaviours, participants completed the Human Factors Attitude Survey (HFAS) pre- and postcourse. Day 1 and day 2 results were compared using repeated measures analysis of variance (ANOVA) and post hoc analysis by pairedsample $t$-tests. Results: Mean overall team scores improved between training days 1 and 2 for both the Mayo Team Scale (day $1=6.17$, day $2=7.41, p=0.13$ ) and Ottawa GRS (day $1=4.04$, day $2=4.36, p=$ $0.19)$, although this did not reach statistical significance. There was a significant difference for average pre-post HFAS scores for all participants $(n=19)$ on day 1 (pre $0.82+0.07$, post $0.89+0.07)$ and post hoc analysis among those same $(n=8)$ participants for day 2 (pre $0.86+$ 0.07 , post $0.90+0.07$ ). Conclusion: TNT training resulted in improved team-based attitudes and a trend toward improved performance between training days 1 and 2, although this pilot study was likely underpowered to detect a significant difference.

$L O P 21$ Utilizing national emergency department quality indicators to evaluate asthma care in an Ontario Local Health Integrated Network 
W. Supapol, PhD, M. Ackerman, MD, A. Worster, MD; McMaster University

Keywords: asthma, emergency department, emergency medicine, quality indicator

Introduction: We applied 1 of ICES consensus list of 48 evidence-based quality indicators for Canadian emergency departments (EDs) to assess and compare the quality of asthma care at 12 Ontario EDs within a single Local Health Integrated Network. Methods: We reviewed approximately 100 randomly selected charts with a diagnosis of asthma at each ED for adult visits occurring between July 2009 and June 2011 for objective measures of lung function including spirometry, peak flow, and $F E V_{1}$. Our two trained data abstractors used standardized data collection procedures and forms, and data were simultaneously double abstracted and agreed upon for accuracy. Triage, ED visit, ED nursing, and allied health notes and reports were examined. We determined the proportion of asthma visits where pulmonary function testing (PFT) was performed with $95 \%$ confidence intervals $(95 \% \mathrm{CI})$ and tested whether the observed proportion was significantly different from a hypothetical standard of $80 \%$ using the binomial proportion test. Results: All 12 hospitals agreed to participate, and we reviewed 1,208 ED asthma visit charts. PFT for asthma visits ranged from $0 \%(95 \%$ CI: $0-3)$ to $41 \%(31-51)$ at the 12 hospitals. All hospitals performed significantly below the expected standard. We assessed whether PFT may have occurred more frequently in the intermediate CTAS levels where PFT may be most useful in determining treatment. However, PFT testing was observed in 0 to $40 \%$ of asthma visits with patients triaged to CTAS level $3(n=656)$ and among patients triaged at CTAS level 2, 3, or $4(n=1,161)$. Conclusion: PFT for asthma care is significantly below the expected standard at all hospitals in the LHIN. As there was no specific location for recording peak flow in charts, it is possible that peak flow may have been performed and not recorded, resulting in an underestimate of PFT.

\section{LOP22 Wake up: head injury management around the clock}

P.R. Atkinson, MD, FCEM, J. Fraser, Bachelor of Nursing, S. Benjamin, RNBN, E. Sproul, RNBN, A. Mehta, B Eng; New Brunswick Trauma Program

Keywords: computed tomography, emergency medicine, head injury Introduction: We aimed to determine whether the time of day and the severity of the injury affected the time to CT scan of patients with head injury presenting to the emergency department at a Level 1 trauma centre. Methods: The study was a retrospective chart review of all head CT scans performed for trauma patients over a 5-year period, identified from the trauma database $(n=904)$. Our primary outcome was time from emergency physician assessment to CT scan. Data were analyzed according to severity of head injury, time of presentation, and disposition using nonparametric tests (GraphPad Prism). Results: Mild head injury (GCS 13-15) median times to CT (68 minutes) were longer than moderate (GCS 9-12; 50 minutes) and severe (GCS 3-8; 53 minutes) head injuries, respectively (Kruskal-Wallis test $p<0.05$ ). There was a significantly longer median time to CT scan overnight (midnight to $8 \mathrm{am}$ ) for all patients (79 minutes) when compared to day/evening presentations (63 minutes; Mann-Whitney test $p<$ 0.0001 ). For clinically significant head injuries (defined as moderate or severe; GCS 3-12), there was also a significantly longer median time to CT scan overnight (59 minutes v. 47 minutes; Mann-Whitney test $p$ $=0.044$ ). For patients who died or went to the operating room or intensive care, there was a trend toward longer median times to $\mathrm{CT}$ overnight (59 minutes v. 55 minutes; Mann-Whitney test $p=0.051$ ). Conclusion: Severity of head injury and arrival at hospital after midnight were associated with significantly longer times to head CT in this Level 1 trauma centre. Further investigation is indicated to determine what factors contribute to this delay.
LOP23 Does use of bedside ultrasound affect mortality outcome in patients who present with undifferentiated hypotension? Preliminary results from Sonography in Hypotension and Cardiac Arrest in the Emergency Department (SHoC-ED) 1

J. Milne, Medical Student, J. Fraser, Bachelor of Nursing, J. Wright, MD, P. Olszynski, MD, H. Lamprecht, MBChB, FCEM, D. Lewis, MBBS, FRCS, FCEM, P.R. Atkinson, MD, FCEM; Dalhousie Medicine, New Brunswick

Keywords: emergency medicine, point-of-care ultrasound, shock Introduction: There is little published evidence of any mortality benefit with point-of-care ultrasound (PoCUS) in patients with undifferentiated nontraumatic hypotension. We have initiated a multicentre randomized controlled trial to assess the impact of the Abdominal and Cardiothoracic Evaluation by Sonography in Shock (ACES) protocol on survival and key clinical outcomes in this group. We report on our first 35 patients recruited. Methods: Screening at triage identified patients with hypotension (SBP $<100$ or shock index $>1$ ) who were randomized to ultrasound or control group (standard care). An initial and secondary diagnosis was recorded at 0 and 60 minutes. A PoCUSaccredited physician performed an ACES protocol scan in the ultrasound group prior to the secondary assessment. The primary outcome measure was 7- and 30-day mortality. The secondary outcome measures included time to normalization of vital signs and acid-base status and correction of initial diagnoses. Results: To date, we have enrolled 35 patients at our primary site. There was a nonsignificant trend toward improved 30-day survival in the PoCUS group (18/20 v. 11/15; OR $3.3,95 \%$ CI 0.51 to $21 ; p=0.367)$. There was no difference in change of secondary diagnosis between the groups $(p=0.69)$. Data collection was complete for the primary and secondary outcome measures other than 4-hour repeat labs (19/35). Conclusion: We are pleased with the initial implementation and believe that SHoC-ED has potential to demonstrate a mortality difference in patients who present with undifferentiated hypotension and may play a role in determining if PoCUS should become a standard of care for this patient group. It is not surprising that preliminary results have not been statistically significant as we have powered the study with a sample size of 400 required.

\section{LOP24}

Creating safety in everyday practice in an urban emergency department

G.S. Hunte, MD, PhD, J.J. Joyce, PhD, W.H. McKellin, PhD, S.B. Sheps, MD, MSc; St. Paul's Hospital

\section{Keywords: emergency medicine, patient safety}

Introduction: "Safety culture" surveys are popular but problematic. We explored how health care providers view and create safety in an urban ED using the "questerview" strategy. Methods: Forty-one ED health care professionals (including nurses, physicians, and emergency staff) and hospital administrators at an inner-city tertiary care hospital completed a standardized patient safety culture questionnaire and then participated in follow-up in-depth interviews and focus groups to reflect on the meaning of their survey responses and prompt narrative exploration of factors that contribute to patient safety. Interviews were electronically recorded and transcribed verbatim. Data were iteratively analyzed for emergent patterns and themes. Results: Reporting of patient safety incidents was perceived as ineffectual ("why bother?" more accounting than learning), with a sense of "no harm, no foul." Speaking up was rarely rewarded. Feedback and learning, if present, were experienced as ad hoc and local rather than systematic and organizational. Threats to safety were normalized ("happens all the time"). Creating "safe space" within constraints of flow, capacity, and resources were sites of negotiation and resistance and perceived to be more a function of individual provider action than inherent in system 
design ("square peg in a round hole"). Providers and staff took pride in providing good care but thought a "bomb had to go off" to force effective cooperation. Front-line providers were frustrated and had a sense of abandonment, embodying professional and departmental rather than organizational identities ("us against them"). Gaps in teamwork, responsibility, and communication at transitions in patient care were considered problematic. Conclusion: These findings reveal organizational features that influence how health care providers create safety for patients in a busy, urban ED but are not sufficiently captured on patient safety culture surveys. Proactive concrete system responses based on understanding of everyday operations, "how the work gets done," offer opportunities for improving the safety of patient care.

\section{Moderated Posters}

\section{MP01 The use of etomidate as an induction agent in trauma patients \\ R. Green, MD, C.S. Hinkewich, MD; Dalhousie University}

Keywords: emergency intubation, emergency medicine, etomidate Introduction: Etomidate has a neutral hemodynamic profile that makes it attractive for emergent intubation. Despite theoretical advantages of etomidate in the trauma patient, data supporting its use are incomplete. This study examined the use of etomidate for emergent intubation in traumatic illness. Methods: This study is a retrospective review of all major adult trauma patients who required intubation at the QEII HSC in Halifax since 2000. Demographics, comorbidities, trauma-specific variables, vitals, length of stay (LOS) in ICU and hospital, ventilation-free days, and mortality were analyzed. Associations between the use of etomidate and 28-day mortality are presented as odds ratios. Logistic models were adjusted for age, injury severity score (ISS), gender, comorbidities, and injury type. The effects of etomidate on other outcome variables were assessed using $t$-tests. Results: A total of 315 patients were included. Patients receiving etomidate were similar to those who did not, including ISS and preintubation blood pressure. There was no difference in ICU LOS, hospital LOS, or ventilation-free days. Twenty-eight-day mortality was higher in the etomidate group $(20.0 \%$ v. $10.8 \%, p=0.04)$. Etomidate was associated with increased mortality, with crude odds of 2.06 (95\% CI $1.08-3.91, p=0.03)$. After adjustment for age, sex, ISS, and comorbidities, the odds ratio was 2.00 (95\% CI $0.91-4.40, p=0.08)$. The survival benefit in the nonetomidate group is seen at day 1 and persists for the remainder of the 28 days. Conclusion: Administration of etomidate for emergency intubation in the trauma patient may be associated with increased mortality. Etomidate should be used with caution in trauma patients requiring intubation. The risk-benefit of etomidate use in this patient population requires further investigation.

MP02 Comparisons of self-reported and validated primary outcomes in a randomized controlled trial of enhanced care in acute wheezing emergencies

B.H. Rowe, MD, MSc, CCFP(EM), C. Villa-Roel, MD, PhD (Candidate), E. Cross, MD (Candidate), L. Rodriguez, MD, PhD (Candidate), S. Couperthwaite, BSc, J. Victor, BScN, M. Bhutani, MD, FRCPC; University of Alberta

Keywords: asthma, COPD, emergency medicine, follow-up, methods Introduction: Most trials of patients discharged from the emergency department (ED) following management of asthma or chronic obstructive pulmonary disease (COPD) examine relapse (unscheduled medical visit for worsening respiratory symptoms) and follow-up (usually with primary care physicians [PCP]) as primary clinical outcomes. The objective of this substudy was to compare self-report with efforts to val- idate outcomes on the results of a controlled clinical trial comparing standard to enhanced care on PCP contact and relapses after ED discharge. Methods: A block-randomized controlled clinical trial was conducted at one $\mathrm{ED}$ in Edmonton, $\mathrm{AB}$, using concealment of allocation and multiple levels of blinding. The physicians of patients with MDdiagnosed asthma or COPD exacerbations were randomized at discharge to receive a standard faxed ED chart (control) or a personalized Lung Attack Alert letter signed by a local opinion leader (intervention). All patients received evidence-based discharge medications (asthma: prednisone and inhaled corticosteroids; COPD: prednisone and antibiotics). Patients' self-reported outcomes (telephone contact) at 30 and 90 days were systematically verified. Agreement was measured by kappa $(\kappa) /$ intraclass correlation coefficient (ICC), and time-to-event analyses used log-rank tests. Results: From 786 screened patients, 136 were consented and 128 were fully enrolled. Overall, 65 received intervention and 63 control interventions; $53 \%$ were females, and the median age was 46 . Follow-up was $84 \%$ at 30 and $71 \%$ at 90 days. Agreement was moderate $(\kappa=0.60)$ for relapses and fair $(\kappa=0.49)$ for PCP visits; ICCs for earliest day of relapse and PCP visit were poor $(0.20$ and 0.35 , respectively). Analyses of time to event between treatments for selfreport and validated outcomes failed to alter statistical significance for both relapse ( $p=0.37$ and 0.31 , respectively) and PCP visits ( $p=0.49$ and 0.98 , respectively); however, the sample size limited this analysis. Conclusion: Overall, outcomes based on patient self-report showed poor to moderate agreement with verified outcomes, and verification resulted in improved data quality. While efforts to validate outcomes are time-consuming and expensive, they should be undertaken as a standard practice to avoid information bias in clinical trials.

\section{MP03 Survival analysis after head injury: is a normal INR reas- suring?}

K. Leclerc-Gagné, MD, J. Chauny, MD, MSc, R. Daoust, MD, J. Paquet, Statistician, D. Gilbert, MD, J. Giguère, MD, N. Gosselin, MD, M. Vincent, MD; Hôpital Sacré-Coeur de Montréal

Keywords: coagulation, emergency medicine, traumatology

Introduction: Intracranial bleeding after head injury is an important issue in the emergency department (ED), and an elevated international normalized ratio (INR) increases this risk. A database study showed an association between normal INRs and the risk of intracranial hemorrhage, with an INR threshold of 1.3 associated with significantly higher risk. The objective of this study was to assess the clinical impact of normal INR $(<1.6)$ in patients with head injury. We compared mortality between patients with an INR $<1.3$ and an INR $\geq 1.3$. Methods: Post hoc analysis of prospective data collected from 3,356 patients seen in a tertiary care ED from March 2008 to February 2011. We included patients aged 16 years old and over with an INR of $<1.6$ and head CT performed within 24 hours of the trauma. We followed these patients until September 2012 (death or survival in the hospital), performed survival analysis using Cox regression and Kaplan-Meier curves, and calculated the hazard ratio (HR). Results: A total of 728 patients were recruited. The mean age was 55.1 years $(\mathrm{SD} \pm 23)$; $65 \%$ were men. Forty patients $(5.5 \%)$ died during follow-up: $7(15.8 \%)$ in the group with an INR $\geq 1.3$ and $33(4.8 \%)$ in the group with an INR $<1.3$. Results showed a significantly increased risk of death in the group with an $\mathrm{INR} \geq 1.3$ : $\mathrm{HR}=2.78(95 \% \mathrm{CI}=1.23$ $6.29), p=0.014$. Conclusion: In patients with head injury and a normal INR $(<1.6)$, a significantly increased risk of death was found in the group with an INR $\geq 1.3$. Therefore, it would be useful to request an INR in patients presenting with a head injury when bleeding is suspected, even in the absence of anticoagulants.

MP04 Survival rates for out-of-hospital cardiac arrest patients transported without a prehospital return of spontaneous circulation 
I. Drennan, BSc, A-EMCA, S. Lin, MDCM, MSc, D. Sidalak, BSc, L. Morrison, MSc, MD, FRCPC; University of Toronto

Keywords: cardiac arrest, emergency medicine, EMS

Introduction: Implementation of the prehospital universal termination of resuscitation (TOR) guideline (terminate without a prehospital return of spontaneous circulation [ROSC] and not EMS witnessed and no shock provided) has reduced the transport rate to hospital. Some services currently use the absence of prehospital ROSC as the single criterion to terminate resuscitation, which may deny transport to potential survivors. This study aims to describe patient and prehospital factors associated with survival to hospital discharge in OHCA patients transported to hospital without a prehospital ROSC. Methods: A retrospective observational study of consecutive, nontraumatic, adult OHCA patients without a prehospital ROSC who met the universal TOR guideline for transport with ongoing resuscitation. Bivariate analyses were used to determine associations of patient and prehospital variables with survival. Results: There were 19,081 EMS-treated OHCA, of whom 3,273 (17.2\%) did not have a prehospital ROSC but met the universal TOR guideline for transport with ongoing resuscitation. Of these patients, 115 (3.5\%) survived to hospital discharge. Survivors were younger (60.0 years; IQR 50.0-70.0 v. 67.0 years; IQR $55.0-78.0, p<0.01)$, with initial shockable rhythms $(83.8 \%$ v. $54.6 \%$, $p<0.01)$, witnessed by EMS $(28.1 \%$ v. $27.6 \%, p=0.01)$ or bystanders $(56.1 \%$ v. $44.8 \%, p=0.01)$, in a public location $(47.4 \%$ v. $29.8 \%, p<$ $.01)$, with no advanced airway $(62.7 \%$ v. $82.1 \%, p<0.01)$. No differences were noted in male gender $(77.4 \%$ v. $74.2 \%, p=0.44)$, bystander CPR $(37.4 \%$ v. $31.2 \%, p=0.28)$, EMS response (5.6 minutes; IQR 4.4-7.3 v. 6.0 minutes; IQR 5.0-7.7, $p=0.22$ ), ALS provider $(74.9 \%$ v. $81.0 \%, p=0.10)$, or academic hospital destination (24.4\% v. $17.8 \%, p=0.07)$. Conclusion: In patients without a prehospital ROSC who met the universal TOR guideline for transport with ongoing resuscitation, $3.5 \%$ survived to hospital discharge. This rate of survival is higher than that defined as medically futile $(<1 \%)$ and suggests that termination of resuscitation should not be based on the absence of prehospital ROSC alone.

MP05 Survival analysis of patients taking dabigatran who consulted an emergency department for acute bleeding: a first alarm O. Arlet, MD, Notebaert, MD, MSc, J. Paquet, Statistician, R. Daoust, MD, M. Vincent, MD, J. Chauny, MD, MSc; Hôpital du Sacré-Coeur de Montréal

\section{Keyword: emergency medicine}

Introduction: The newly approved oral anticoagulant dabigatran has no effective antidote. We therefore suspected an overall increase in mortality in patients presenting at the emergency department (ED) with a bleeding complication on dabigatran compared to warfarin or aspirin. Methods: We conducted a post hoc analysis on a database of all patients admitted to a tertiary care ED with any kind of bleeding or suspicion of one from March 2011 to August 2012 who were taking dabigatran, warfarin, or aspirin. The primary end point was long-term survival. Patients were censored at death or at the end of the study period (December 7, 2012). We performed a Cox proportional hazard model, controlled for age, to calculate the hazard ratio (HR) for dabigatran versus warfarin and one for warfarin versus aspirin. Statistical significance was set at alpha $=0.05$, and results are presented with $95 \%$ confidence intervals $(95 \% \mathrm{CI})$. Results: A total of 943 patients met the inclusion criteria with a mean follow-up period of 1 year. The mean age was 74.3 years and $50.4 \%$ were men. A total of 108 deaths (11.5\%) were recorded within the follow-up period: 8 (25\%) for dabigatran compared to $44(12.6 \%)$ for warfarin and $56(9.9 \%)$ for aspirin. The mortality risk for patients on dabigatran was significantly higher than for patients on warfarin: $\mathrm{HR}=2.1(95 \% \mathrm{CI}: 1.0-4.5), p=0.05$ after controlling for age. Aspirin had a lower (but not statistically significant) mortality risk compared to warfarin: HR $=0.75(95 \% \mathrm{CI}$ : $0.50-1.14), p=0.18$ after controlling for age. Conclusion: The results showed higher overall mortality in patients who presented to the ED with a bleeding complication and were taking dabigatran compared to warfarin or aspirin. Physicians should be aware of the potential higher mortality with dabigatran over warfarin when treating a bleeding patient. However, this was a single-centre retrospective analysis with a small number of patients taking dabigatran $(n=32)$, and further studies are needed to corroborate the results.

MP06 Does neighbourhood matter? How location influences cardiac arrest outcomes and predictability of the Utstein factors J.E. Buick, BScKin, J.G. Ray, MD, MSc, FRCPC, A. Kiss, PhD, L. Morrison, MSc, MD, FRCPC; Institute of Health Policy, Management and Evaluation, University of Toronto

Keywords: cardiac arrest, emergency medical services, emergency medicine, Geographic Information System

Introduction: The Utstein factors only predict $72 \%$ of out-of-hospital cardiac arrest (OHCA) survival variability (AUC: 0.86). Previous studies demonstrated geographic variations in both OHCA incidence and outcomes, while others have related these differences to ethnic and socioeconomic measures. The objective of this study was to determine the role of neighbourhoods in OHCA survival and predictability in a Canadian setting. We hypothesized that there are neighbourhood factors associated with survival and variability in cardiac arrest outcomes. Methods: All treated OHCA between April 2006 and March 2010 were mapped into census tracts (CT) using Geographic Information System analysis. Based on their home address, patients were assigned neighbourhood factors extracted from the 2005 Canadian census, including instability, dependency, deprivation, ethnic diversity, crime rate, and density of family physicians. Hierarchical logistic modeling was performed to assess which factors were associated with survival. Results: A total of 4,626 OHCA were eligible for enrolment and contained the required information for geographic mapping. Survival to hospital discharge was $4.3 \%$. The CT survival varied between 0 and 50\% (IQR: $0.0-7.7$ ), where $68.4 \%$ had $0 \%$ survival. The Utstein factors predicted $85.4 \%$ (95\% CI: $81.0-89.6)$ of survival variability. After adjusting for the Utstein factors, only the ethnic concentration of the CT was associated with survival. Compared to only the Utstein factors, inclusion of CT variables increased the predictability of the Utstein factors to $87.2 \%$ (95\% CI: 83.4-91.2, $p=0.005$ ). Conclusion: Although neighbourhood differences in OHCA outcomes exist, this difference contributes minimally to the Utstein factors' prediction of survival. Since bystander resuscitation efforts are the most powerful predictors of survival, perhaps neighbourhood differences in bystander resuscitation efforts may address the gap in the Utstein predictability.

MP07 The reliability and validity of roadside observations of cyclists and their helmet use

R. Chetram, BSc (Candidate), C. Tarun, BSc (Candidate), B. Holroyd, $\mathrm{BScN}$ (Candidate), C. Villa-Roel, MD, PhD (Candidate), D. Voaklander, PhD, B. Hagel, PhD, A. Nettel-Aguirre, PhD, B.H. Rowe, MD, MSc, CCFP(EM); University of Alberta

Keywords: emergency medicine, helmets, prevention, reliability/validity

Introduction: Bicycling is a popular recreational and transportation action in Canada; however, injuries can result in health professional visits, ED presentations, hospitalization, and death. Bicycle helmets have been shown to protect the brain and face/head from serious 
injuries, and legislation in developed countries has increased the use of helmets by bicyclists, especially in children. To study bicycle helmet prevalence, many researchers have used roadside observation by trained staff. This study was designed to examine the reliability and validity of helmet observations. Methods: A prospective observational study was conducted in the summer of 2012 in Edmonton and St. Albert, Alberta. Roadside observation sites were selected from previous surveys. Two observers independently documented approximate age, helmet use, helmet type, position on head, and strap tightness on all passing cyclists to determine reliability. Validity was measured by comparing the observations of those cyclists wearing helmets who stopped to participate in a roadside interview remote from the original observation site. Reliability and validity were reported using simple agreement, kappa $(\kappa)$, and weighted kappa $(\kappa \mathrm{W})$ with $95 \%$ confidence intervals (CI). Results: Overall, 562 observations were made by three observers. The most common observation location was a bicycle path (70\%); however, schools (12\%) and other locations (18\%) were also sampled. Helmet use was $61 \%$ in the observed sample. Interobserver reliability $(n=562)$ was excellent for sex $(\kappa=0.96$; $95 \%$ CI: 0.92 , $0.98)$, age ( $\mathrm{\kappa W}=0.96 ; 95 \%$ CI: $0.94,0.98)$, helmet use $(\kappa=0.99 ; 95 \%$ CI: $0.96,1.0)$, and helmet fit $(\kappa=0.94 ; 95 \%$ CI: $0.86,0.97)$. Interobserver validity $(n=253)$ was good for sex $(\kappa=0.71 ; 95 \%$ CI: 0.61 , $0.79)$ and age ( $\mathrm{KW}=0.69 ; 95 \% \mathrm{CI}: 0.621$ to 0.762$)$ and moderate for helmet fit $(\kappa=0.43 ; 95 \%$ CI: $0.27,0.57)$. Conclusion: Observations regarding cyclist demographics, helmet use, and helmet fit by trained roadside observers are reliable; however, observers underestimate helmet fit compared to roadside testing. Overall, bicycle roadside observations remain a reliable and valid research method for cyclist and helmet use surveillance.

\section{MP08 Deos neighbourhood really matter? How location influ-} ences bystander CPR rates

J.E. Buick, BScKin, J.G. Ray, MD, MSc, FRCPC, A. Kiss, PhD, L. Morrison, MSc, MD, FRCPC; Institute of Health Policy, Management and Evaluation, University of Toronto

Keywords: cardiac arrest, emergency medical services, emergency medicine, Geographic Information System

Introduction: Numerous studies have investigated determinants of bystander cardiopulmonary resuscitation (CPR) for out-of-hospital cardiac arrest (OHCA) patients. Socioeconomic status and ethnicity have been linked to who receives bystander CPR, and recent studies have showed geographic differences in CPR rates. Research from other disciplines has suggested that location is a strong determinant of health and health-related interventions and outcomes. Therefore, the objective of this study was to determine the role of neighbourhoods in bystander $\mathrm{CPR}$ rates in a Canadian population. We hypothesized that neighbourhood factors are associated with bystander CPR rates. Methods: All treated OHCA within the City of Toronto between April 2006 and March 2010 were included. Geographic Information Systems were used to assign patients to census tracts (CT) based on their pick-up address and linked to neighbourhood factors (instability, dependency, deprivation, ethnic diversity, crime rate, and density of family physicians). Bivariate and hierarchical logistic modeling was performed to assess which factors were associated with bystander CPR. Results: A total of 5,139 OHCA were eligible for enrolment and contained the required information for geographic mapping. The bystander CPR rate was $41.1 \%$. The bystander CPR rate varied across census tracts $(0$ 92\%, IQR: $25.0-50.0 \%$ ), where $10 \%$ had a bystander CPR rate lower than $15 \%$. The Utstein factors predicted $22.0 \%$ (95\% CI: $18.6-25.2 \%)$ of bystander CPR variability. In a bivariate analysis, the CTs deprivation and density of family physicians were associated with bystander CPR. After adjusting for individual characteristics, no CT-level variables were associated with bystander CPR. Compared to only the
Utstein factors, inclusion of CT variables did not increase the predictability of the Utstein factors $(p=0.09)$. Conclusion: Deprivation and the density of family physicians were associated with higher rates of bystander CPR. However, after adjustment for patient characteristics, neighbourhoods were not associated with CPR. Future research should explore the relationship between location and witnessed status and its role in bystander CPR.

\section{MP09 Knowledge translation effects of AHA CPR guidelines in first responder programs in British Columbia}

E.N. Vu, MD, CCP, FRCPC, DAvMed, B.T. Livingstone, BSC, D.E. Griesdale, MD, MPH, FRCPC, O.A. Olson, BHSc, A. Holmes, MD, FRCPC; Department of Emergency and Critical Care Medicine

Keywords: AHA guidelines, compression rate, CPR ratio, emergency medicine

Introduction: The AHA publishes updated guidelines on CPR and emergency cardiac care (ECC) every 5 years. A striking finding of the 2005 consensus conference was the contrast of data that showed the role of early, high-quality CPR increasing survival rates from cardiac arrest. "High-quality CPR" was emphasized: push hard, push fast, allow full recoil, minimize interruptions, and defibrillate promptly when appropriate. We sought to characterize the translation of the 2005 AHA guidelines on first responder (FR) CPR programs in British Columbia (BC). Methods: Global Medical Services (GMS) provides medical oversight for $>150$ professional/volunteer FR programs. Each cardiac arrest in which an AED is used is downloaded to headquarters and reviewed by a physician or paramedic. Software from the AED manufacurter exports data on CPR ratio, compression ratio, and compression rate. Results: Over a 10-year period (01/01/01-12/15/11), we reviewed 3,004 cardiac arrests. Mean, median, and modes were exported for each of $\mathrm{CPR}$ ratio, compression ratio, and compression rate. There was a dramatic increase in CPR and compression ratios in 2007, 2 years after the 2005 update emphasizing high-quality CPR: rates rose from $42.4 \%$ and $47.3 \%$, respectively, in $2001-2006$, to $71.2 \%$ and $73.4 \%$, respectively ( $p<0.001$ ), in $2007-2011$. The compression rates showed a steady climb, exceeding the prescribed 100 compressions per minute from 2001 to 2011. Conclusion: These data demonstrate remarkable improvements in CPR and compression ratios after the release of the 2005 AHA guidelines. However, it demonstrates a notable lag in knowledge translation, with almost a 2-year delay in improvements of CPR and an unexpected progressive overshoot of the prescribed CPR compression rate from 2001 through to 2011. Our data provide educators with a metric with which to focus medical education and clarification of recommendations from the AHA since 2005

MP10 Utility of outpatient cardiac stress tests in low-risk chest pain patients presenting to the emergency department

J. Pace, MD (Candidate), E. Brennan, Medical Doctor, R.J. Brison, MD, FRCPC, E.P. Hess, MD, MSc; Department of Emergency Medicine, Queen's University

Keywords: acute coronary syndrome, emergency medicine, exercise stress test, low-risk chest pain

Introduction: Outpatient cardiac stress testing, to rule out acute coronary syndromes (ACS), is arranged often for chest pain patients assessed in the emergency department (ED) and discharged home. Recent literature suggests that these tests are predominantly negative in patients deemed low-risk for ACS and hence may contribute little to clinical decision making in post-ED follow-up. We assessed the utility of outpatient cardiac stress tests in guiding medical management for patients whose risk for ACS was assessed using the North American Chest Pain Rule (NACPR). Methods: This study is a secondary analy- 
sis of data collected for the derivation of the NACPR (May 2009August 2010), enhanced with 1-year follow-up for outcomes by medical record review. Existing study data on participants from the Kingston, ON, site permitted classification by the NACPR as low risk or not low risk. All who had outpatient cardiac stress testing, by any modality, were identified. Information on results of testing and hospitalbased investigation and management was abstracted from the single electronic patient care system serving the two acute care hospital systems in our community of 150,000 . Primary outcome measures included (1) all initial and subsequent interpretations of cardiac stress testing and (2) documented changes in medical management for ACS, up to 1 year post ED encounter. Medical management change was defined as an alteration to the patient's cardiac medications for ACS management or a revascularization procedure within 1 year of the incident ED encounter. Results: A total of 1,070 participants were identified; 196 of these $(19 \%)$ had outpatient cardiac stress testing. Thirtytwo patients $(16 \%)$ had a positive test result. Forty-four of those tested $(22 \%)$ were classified as low risk with only one positive test $(2.3 \%$; $95 \%$ CI $0,3.9)$ leading to a change in cardioprotective medications. There were five positive stress tests $(11 \%)$ with three deemed false positives in cardiology follow-up. One patient had no follow-up data on record. Of 152 classified as not low risk, 27 (17.8\%) had positive stress tests. Of these, 11 were deemed false positive and 16 were positive and associated with a management change. Conclusion: This study suggests that ED chest pain patients classified as low risk by the NACPR do not require outpatient cardiac stress testing. While based on a moderate-sized, single-centre study sample, we estimate that more than $20 \%$ of outpatient stress tests may be redundant.

MP11 Air medical transfer of patients in preterm labour: current treatments and tocolytics provided in Ontario

K.G. Thompson, MD, C. Vaillancourt, MD, MSc, FRCPC, CSPQ, S. Moore, MD, R. MacDonald, MD, University of Ottawa, Department of Emergency Medicine

Keywords: air medical, emergency medicine, pregnancy, transport Introduction: Patients with preterm labour (PTL) who are transferred to tertiary care centres are frequently given tocolytic medication to prevent delivery during transport. There are currently no Canadian guidelines to support the effectiveness and safety of this practice. We sought to determine tocolytic use, diagnostic testing, and patient-oriented outcomes for women in PTL transported to tertiary care facilities in Ontario. Methods: We performed a health record review of consecutive PTL patients ( $<38$ weeks' gestation) transported by Ontario's air ambulance service to a tertiary care facility between Jan. 1, 2006, and Jan. 1, 2011. Our primary outcome was the type of tocolytics used and their ability to decrease contractions and prevent in-flight deliveries. Secondary outcomes included adverse events (defined a priori), use of standard treatments (corticosteroid, antibiotic), and use of predictive tests (cervical length measurement, fetal fibronectin). We piloted our data extraction form, and a second trained reviewer also extracted data elements for $10 \%$ of the charts randomly selected to determine interrater agreement. We report descriptive statistics. Results: Of the 510 health records reviewed, 488 met all inclusion criteria with the following characteristics: mean age 26.1, mean gestational age 31.2 weeks, mean transport time 1:20 (hr:min), $61.0 \%$ multiparous, $13.3 \%$ twins, mean initial dilatation $1.8 \mathrm{~cm}$, contraction $<8$ minutes apart $67.7 \%$, and $66.8 \%$ of transfers from northern Ontario. Tocolytics were used in 206 (42.2\%), with NSAIDs and nitroglycerin being used most frequently. Among the 199 (40.8\%) patients for whom a change in contractions was documented, they decreased in 78/116 (67.2\%) patients who received tocolytics compared to $37 / 83(44.6 \%)$ of those who did not. Adverse events were documented in 67 (14\%) patients (most com- monly tachycardia 5.8\%), and $11(2.3 \%)$ patients delivered during transport, 4 (36.4\%) of whom received tocolytics. Steroids and antibiotics were appropriately used in $268(54.9 \%)$ and $286(58.6 \%)$ of patients, respectively, and predictive testing was performed in only 19 $(3.9 \%)$ patients. Conclusion: We observed great variation in the choice of tocolytic drug used, with NSAIDs being most commonly administered. Practice variation suggests a need for clinical practice guidelines that could increase the use of recommended therapies and predictive tests in transport of PTL patients.

\section{MP12 Police documentation of alcohol involvement in hospital- ized injured drivers}

J.R. Brubacher, MD, H. Chan, PhD, R. Purssell, MD, M. Fang, MSc, D. Brown, MD; University of British Columbia

Keywords: emergency medicine, impaired driving, motor vehicle crashes

Introduction: Injured drivers with blood alcohol concentration (BAC) above the legal limit are rarely convicted of impaired driving. In this study, we compare police documentation of alcohol involvement with BAC measured in hospital. Our objectives are to determine how often police document alcohol involvement in injured drivers with $\mathrm{BAC}>$ $0.05 \%$ and identify factors that influence police documentation of alcohol involvement. Methods: We included injured drivers (1999-2003) admitted to a $\mathrm{BC}$ trauma centre or tertiary care ED. We used probabilistic linkage to obtain police collision reports. Police indicated alcohol involvement if they documented that alcohol contributed to the crash or if the driver received an administrative sanction or criminal conviction for impaired driving. The proportion of drivers for whom police indicated alcohol involvement was determined relative to age, gender, BAC levels, crash severity, and crash characteristics. Multivariate logistic regression was used to identify factors associated with police indication of alcohol involvement. Results: A total of 2,410 injured drivers (73.5\% male) were matched to a police report; 857 (35.6\%) had BAC > 0 and $736 / 857$ (85.9\%) had BAC $>0.05 \%$ (the BC legal limit). Police indicated alcohol involvement in 530/736 (72.0\%) drivers with BAC > $0.05 \%$. The following factors were associated with police indication of alcohol involvement: (1) increasing BAC, (2) prior impaired driving record, (3) single-vehicle crashes, (4) nighttime crashes, and (5) traffic violations or unsafe driving actions. Conclusion: Police recognized and documented alcohol involvement in $72 \%$ of injured drivers with $\mathrm{BAC} \geq$ $0.05 \%$. Alcohol involvement is more commonly documented at higher BAC levels, in nighttime or single-vehicle crashes, for drivers who committed traffic violations, drove unsafely, or had a prior impaired driving record. The low conviction rate of injured impaired drivers is not due to police inability to recognize alcohol involvement.

MP13 An emergency department sepsis protocol decreases mortality in septic pneumonia patients

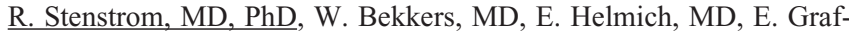
stein, MD, W.W. Lee, MD, D. Harris, MD, PhD, F.X. Scheuermeyer, MD, G.S. Hunte, MD, PhD; St. Paul's Hospital

Keywords: emergency medicine, pneumonia, sepsis

Introduction: The adaptation of sepsis protocols in emergency departments (EDs) is widespread. Most studies in this area have reported a decrease in sepsis mortality associated with protocol implementation. We hypothesized a mortality benefit associated with sepsis protocol implementation in ED patients with pneumonia and sepsis. Methods: This was a retrospective cohort study undertaken at a tertiary care inner-city hospital with 65,000 patient visits yearly. Inclusion criteria were (A) patients admitted from ED to hospital diagnosed with pneumonia who met criteria for sepsis, (B) blood cultures ordered in the 
emergency department, (C) were not DNR, (D) $\geq$ two SIRS criteria Intervention: A multipronged protocol for rapid sepsis recognition and treatment. Main Outcome: Sixty-day mortality. Subjects were identified from the ED database and then underwent extensive, blinded chart review by trained research assistants. Results: A total of 386 patients (193 before and 193 after) met the criteria in the 2 years before and after protocol implementation. Mean age was 72.4 years $(\mathrm{SD}=15.8$ years), and $60.1 \%$ of patients were male. Sixty-day mortality decreased from 17.1 to $6.7 \%(p=0.003)$ after the use of the protocol (absolute risk reduction $=10.4 \%$, number needed to treat $=9.6$ patients). Using multivariable logistic regression to control for patient comorbidities and age demonstrated a significant effect of protocol implementation (odds ratio of death in 60 days $0.32,95 \%$ CI $0.11-0.81$ ). Conclusion: The implementation of a sepsis protocol in the emergency department was associated with a $10.4 \%$ absolute reduction in 60 -day mortality in septic pneumonia patients.

MP14 CT scan versus ultrasound for the diagnosis of appendicitis: what is the impact on emergency department length of stay? R. Simard, MD, J.S. Lee, MD, MSc, S.C. Brooks, MD MHSc, S.H. Gray, MD, FRCPC; University of Toronto

Keywords: appendicitis, diagnostic imaging, emergency medicine, length of stay

Introduction: Computed tomography (CT) scan and ultrasound are commonly used to diagnose appendicitis. $\mathrm{CT}$ is more accurate and is hypothesized to be the faster test; however, ultrasound avoids radiation. The goal was to determine if emergency department (ED) length of stay (LOS) was shorter if patients with appendicitis were first investigated by CT scan. Methods: A retrospective chart review was performed at an academic hospital in Toronto, Ontario, from January 2007 to August 2010. All adult patients with appendicitis diagnosed in the ED on CT scan or ultrasound were included. Multivariable regression was used to determine predictors of ED LOS. Also, ED physicians were surveyed regarding their current imaging practice for appendicitis. Results: A total of 364 appendicitis patients were included, with 251 undergoing CT first and 105 undergoing ultrasound first. Fortyeight ultrasound first patients also received CT. CT first had mean LOS of 10.9 hours ( $95 \%$ CI 10.3-11.5), and ultrasound first had mean LOS of 11.4 hours $(95 \%$ CI $10.4-12.5)$. The 68 patients with ultrasound only had the shortest mean LOS of 9.0 hours (95\% CI 7.9-10.1), while ultrasound and then CT had a mean ED LOS of 14.7 hours $(95 \% \mathrm{CI}$ 13.0-14.7). In 11 of the positive ultrasounds, General Surgery ordered a CT scan, which confirmed that $10 / 11$ patients had appendicitis, adding to ED LOS. Multivariable regression-confirmed CT first was associated with a longer LOS when controlling for whether patients received both ultrasound and CT scan; $72.2 \%$ of ED physicians surveyed stated that ordering a $\mathrm{CT}$ scan first would result in the shortest ED LOS. Conclusion: CT first did not have a shorter ED LOS. ED LOS was shortest if ultrasound was first ordered unless the patient had a CT after ultrasound. Further research should identify patients more likely to have indeterminate ultrasounds and assess whether confirmatory $\mathrm{CT}$ is needed when ultrasound demonstrates appendicitis.

\section{MP15 Do satisfaction surveys sent by email after emergency care} engender bias?

T. Principi, MD, K. Boutis, MD, S.C. Porter, MD; The Hospital for Sick Children

Keywords: electronic mail, emergency medicine, questionnaire Introduction: Increasing access and use of electronic forms of communication may provide a useful mechanism to acquire postvisit information. Variation in access and actual engagement with electronic communication postdischarge may impact the value and generalizability of information obtained. The objectives were to determine the proportion of parents participating in an electronic survey following discharge from the emergency department (ED) and to evaluate the variation in email access and survey engagement. Methods: We conducted a prospective cohort study over a 4-week period on parental access and engagement with electronic communication postdischarge from an urban, tertiary pediatric ED. A single-page form was used to invite parents to provide their email address. Emails with a survey link were sent out weekly with up to two additional reminders. Patient information from each visit was extracted and merged with participation data to evaluate variation in email access and survey participation. Results: A total of 3,869 ED visits occurred during the study period. Completed forms were returned for 3,514 (90.8\%) visits; 2,846 (81.0\%) parents were willing to provide an email address, $203(5.8 \%)$ parents did not have an email address, and $465(13.2 \%)$ parents used email but declined to share it. A survey was completed for 1,038 (29.5\%, 95\% CI $28.1-$ 31.1) patient visits. Compared to those with email, parents without email were more likely to have older children (5.2 years v. 4.0 years, $p$ $=0.01)$ and live in an urban centre $(77.2 \%$ v. $65.8 \%, p<0.001)$ but less likely to speak English $(85.4 \%$ v. $93.7 \%, p<0.001)$. Those who completed the survey were more likely to speak English $(94.6 \%$ v. $92.6 \%, p$ $=0.03$ ). No variations in email access and survey engagement were seen with respect to acuity, disposition, and length of stay. Conclusion: An electronic survey holds promise to produce representative data on the English-speaking population receiving care in the ED.

\section{MP16 Prehospital STEMI diagnosis and decision of transport reorientation by the UCCSPU}

A.B. Tanguay, MD, MSc, D. Hébert, MSc, G. Foldes-Busque, PhD, R. Fleet, MD, PhD; CSSS Alphonse-Desjardins

\section{Keywords: emergency medicine, prehospital, STEMI}

Introduction: The Unité de coordination clinique des services préhospitaliers d'urgence (UCCSPU) is a clinical entity modeled after the concept of base hospital. The UCCSPU's primary mission is to ensure better quality prehospital care for users of emergency medical services (EMS) in regions of the province of Québec. A telehealth system is used (transmission of continuous 12-lead ECG monitoring, vital signs, bidirectionnal on-screen communication, mobile phone) to provide medical support remotely to emergency medical technicians (EMTs). Diagnosis of STEMI is made by an emergency physician. We assess decision of transport orientation for percutaneous coronary intervention (PCI) in accordance with American Heart Association/Canadian Cardiovascular Society (AHA/CCS) standards. Methods: The study collected retrospective data from a cohort of patients with the remote diagnosis of STEMI, from July 2006 to December 2012. The data were abstracted from the administrative and clinical database of the UCCSPU by the nurse coordinator and verified by a physician supervisor. Results: The UCCSPU assessed 43,715 patients. Of those, 670 STEMIs were diagnosed, and $69.7 \%$ were directed to a cathlab facility. Of those 467 STEMIs transported to cathlab facilities, $99 \%$ arrived at destination in less than 60 minutes. The decision to redirect patients to cathlab facilities was made on route, bypassing the nearest emergency department (ED). A total of 203 STEMIs not meeting the criteria for PCI were transported to the nearest ED. Using the continuous 12-lead ECG monitoring, 48 of the 670 STEMIs were diagnosed later in the ambulance, even if their first ECG was normal. Conclusion: A prehospital telehealth system facilitates transport orientation to meet STEMI treatment guidelines. There are plans to extend the service province-wide.

MP17 Evaluating missed opportunities in identification of traumatic intracranial hemorrhage in the emergency department: a novel safety strategy? Results from a multicentre study 
S. Jones, MD, MHSc, A. McRae, MD, J. Bastian, MD, K. Lonergan, BSc, D. Wang, PhD, E. Lang, MD, University of Calgary

Keywords: emergency medicine, intracranial hemorrhage, patient safety

Introduction: Patients presenting to the emergency department (ED) with head trauma are often diagnosed with traumatic intracranial hemorrhage (ICH). Clinical decision rules have been developed and validated to help ED physicians identify patients with ICH. Even with these rules, there remains a small risk of delayed identification of $\mathrm{ICH}$, especially in anticoagulated patients. The purpose of our study is to identify any missed opportunities for improved patient safety that contribute to delayed diagnosis of ICH in patients presenting to the ED with head trauma. Methods: We conducted a retrospective case series in Calgary, Alberta. We relied on data drawn from electronic health record-derived sources and compiled over 21 months (2011/2012) from three tertiary care EDs. We identified all patients who presented to a Calgary-area ED with a diagnosis of traumatic ICH determined by International Classification of Diseases (ICD-10) and coded by a nosologist. Other variables collected were previous presentation within 72 hours with or without neuroimaging, age, sex, INR, and platelet count. Results: Over a 21 -month period, 624 patients presented to Calgary-area EDs with head trauma and were diagnosed with traumatic ICH. Of these patients, 552 were admitted to hospital, while 72 were discharged home with advice. Among all patients in our sample, 13/624 (2.08\%; 95\% CI 0.96-3.2) had an ED visit within the 3 days preceding their diagnosis of $\mathrm{ICH}$, representing either a missed or delayed presentation of ICH. Of the 13 patients with a delayed identification of ICH, 8 had negative neuroimaging results on initial presentation, 5 did not have any imaging at their earlier ED visit, and 7 were eventually admitted to hospital. Of these 13 patients, 11 were over age 65 , none had an elevated INR, all had normal platelet counts, and none died before admission to hospital. Conclusion: Among patients diagnosed with traumatic ICH, several patients had an ED visit within the preceding 3 days, highlighting the potential for missed diagnosis of $\mathrm{ICH}$ and raising the concern about incomplete evaluations of patients with head trauma. There was no correlation between delayed identification of ICH and patients' INR, platelet count, or death. Retrospective evaluations of data can provide insightful information into missed opportunities of care, which can be used to improve patient safety and quality of care.

\section{MP18 Validation of the Blatchford bleeding score and the Rockall score in cancer patients presenting to the ED with upper gastroin- testinal bleeding}

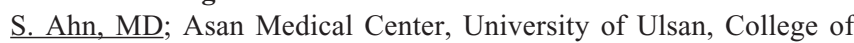
Medicine, Seoul, Korea

Keywords: Blatchford score, emergency medicine, upper gastrointestinal bleeding

Introduction: Several risk scoring systems for upper gastrointestinal bleeding (UGIB) have been developed in an attempt to stratify risk for poor outcomes. The Blatchford score is based on clinical and laboratory variables to predict need for clinical interventions in (UGIB). The primary object was to evaluate the Blatchford score with clinical and full Rockall scores in patients with active cancer presenting to the emergency department (ED) with UGIB. The secondary object was to assess the accuracy of the Blatchford score at a different source of UGIB; cancer bleeding versus nonmalignant lesions. Methods: We reviewed and extracted data from electronic medical records on patients with active cancer presenting to the ED from January 2009 to December 2011. Clinical interventions included blood transfusion, therapeutic endoscopy, angiographic intervention, and surgery. Results: Of the 225 patients included, 197
(87.6\%) received interventions. Comparing the area under receiver operator curves, the Blatchford score $(0.86,95 \%$ CI $0.77-0.95)$ was superior to the clinical Rockall $(0.67,95 \%$ CI $0.55-0.79)$ and full Rockall $(0.72,95 \%$ CI $0.61-0.83)$ scores in predicting interventions. When a score of 2 or less was counted as negative, sensitivity of 0.99 and specificity of 0.54 were calculated. When the patients were separated according to the source of UGIB, sensitivity and specificity were not changed. In UGIB from nonmalignant lesions, the Blatchford score had 1.00 sensitivity and 0.53 specificity. Cancer bleeding showed 0.99 sensitivity and 0.55 specificity. Conclusion: The Blatchford score outperformed both Rockall scoring systems in predicting intervention in patients with active cancer. The source of bleeding was not an important factor in the score performance. The Blatchford score has a very good sensitivity. However, suboptimal specificity limits its role as the sole means of decision making in cancer patients with UGIB.

MP19 The incidence of clinically significant biphasic reactions in emergency department patients and the effectiveness of corticosteroids as prophylaxis

B.E. Grunau, MD, J. Li, BSc, T. Yi, R. Stenstrom, MD, PhD, M.O. Wiens, PharmD, E. Grafstein, MD, R. Schellenberg, MD, F.X. Scheuermeyer, MD; St. Paul's Hospital and UBC Department of Emergency Medicine

Keywords: anaphylaxis, biphasic reaction, emergency medicine Introduction: Biphasic reactions are a feared complication of allergic reactions by emergency physicians. The incidence of subsequent anaphylaxis in the pattern of a biphasic reaction is unknown. In spite of a lack of evidence, corticosteroids are commonly used to prevent biphasic reactions. We sought to determine the incidence of clinically significant biphasic reactions (CSBRs) and the benefit of corticosteroids as prophylaxis. Methods: All consecutive adult patient encounters with an emergency department (ED) discharge diagnosis consistent with allergic reaction were retrospectively identified from two urban teaching hospitals between April 2007 and January 2012. Encounters were classified as "anaphylactic" (as defined by NIAID/FAAN) or otherwise as "allergic reaction." Subsequent ED visits to any of the six EDs in the region within a 7-day period were identified. A comprehensive chart review was conducted and data collected on each index and all subsequent visits. CSBRs, defined as recurrent signs and/or symptoms satisfying the definition of anaphylaxis, were identified. Results: A total of 2,995 index encounters were reviewed to yield 486 anaphylactic and 2,287 allergic reactions. There were 307 subsequent visits. Epinephrine and antihistamines were used in $23 \%$ and $83 \%$ patients, respectively. Corticosteroids were used in the ED in $44 \%$ and prescribed at discharge in $31 \%$. Six CSBRs were identified $(0.22 \% ; 95 \% \mathrm{CI}=0.1-0.47)$. When analyzing the "anaphylactic" and "allergic reaction" groups separately, CSBRs occurred in three $(0.62 \% ; 95 \% \mathrm{CI}=0.21-1.8)$ and three $(0.14 \% ; 95 \% \mathrm{CI}=0.04-0.38)$ patients, respectively. Five patients with CSBRs were treated with corticosteroids in the ED and three postdischarge. CSBRs occurred 16 minutes to 6 days after the initial ED presentation, with $67 \%$ post-ED discharge. There were no fatalities $(95 \% \mathrm{CI}=0-0.014)$. Conclusion: CSBRs are extremely rare. There was no benefit seen with the use of steroid administration. Prolonged ED monitoring appears to yield little benefit in this patient population.

MP20 The utility of serum lactate ordered in the emergency department in diagnosing mesenteric ischemia: a 3-year retrospective review

M. Ahmed, MD, CCFP(EM), S.L. McLeod, MSc, A. Schappert, BSc, J. Dreyer, MD, FRCPC; The University of Western Ontario 
Keywords: emergency medicine, ischemic bowel, mesenteric ischemia, superior mesenteric artery

Introduction: Mesenteric ischemia is a life-threatening condition that is difficult to diagnose as the presenting complaint is often nonspecific abdominal pain. Diagnostic laparotomy and computed tomography (CT) angiography are invasive and expensive and not always immediately utilized. The objective of this study was to evaluate the utility of serum lactate and other investigations in diagnosing mesenteric ischemia. Methods: A retrospective chart review was conducted for a random sample of adult ( $\geq 18$ years) patients admitted from the emergency department (ED) of an academic tertiary care centre (annual census 125,000 ) with an inpatient diagnosis of mesenteric ischemia between 2008 and 2011. Data were extracted by trained research personnel using a standardized data collection tool. Results: One hundred cases were randomly selected; 17 were excluded, leaving 83 patients for analysis. Of the 30 patients (36\%) with mesenteric ischemia, $21(70 \%)$ were confirmed by laparotomy and $9(30 \%)$ were diagnosed by a combination of clinical findings and abdominal CT. Mean (SD) age was 74 (15) years, and 17 (57\%) were female. Fifty-three patients had other diagnoses; 33 (62\%) had ischemic colitis, and 7 (13\%) patients were diagnosed with small bowel obstruction. Twenty-seven of $30(90 \%)$ patients with mesenteric ischemia had abdominal CT. Of these, $8(30 \%)$ were interpreted as having nonspecific findings. Only $2(7 \%)$ had CT angiography. Sixteen of $30(53 \%)$ patients with mesenteric ischemia had a serum lactate drawn in the ED. There was no difference between the mean serum lactate for patients diagnosed with mesenteric ischemia and those with other diagnoses ( 3.6 v. $2.9 ; \Delta 0.7 ; 95 \% \mathrm{CI}:-2.3,0.9)$. Of the 30 patients with mesenteric ischemia, $1(3 \%)$ had gross blood per rectum and $3(10 \%)$ had a positive fecal occult blood test. Conclusion: Serum lactate has poor sensitivity and poor specificity in patients with mesenteric ischemia. Emergency physicians should not rely solely on laboratory markers or nonangiographic abdominal CT when considering the diagnosis of acute mesenteric ischemia.

\section{MP21 The "Do not hospitalize" patient: how often do they present} to the ED?

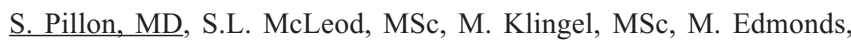
MD, FRCPC, MSc; The University of Western Ontario

Keywords: do not hospitalize, emergency medicine, nursing home Introduction: Nursing home residents use significant health care resources in the emergency department (ED). These transfers are also onerous to patients who may not want aggressive medical management. To avoid unwanted hospital transfers, many patients have do not hospitalize (DNH) advance directives. Most nursing homes outline these directives into four levels of care. Level 1 involves comfort measures only, while level 2 involves treatment of acute conditions in the nursing home. Levels 3 and 4 entail transfer to hospital for acute care without CPR and transfer to hospital with full resuscitation, respectively. The first two levels are considered DNH orders. The objective of this study was to determine the proportion of nursing home patients transferred by emergency medical services (EMS) to the ED who had a DNH directive. Methods: This was a prospective cohort study of nursing home patients transported by EMS to one of two EDs of a tertiary care centre (annual census 125,000) over a 6-week study period. Patients from a retirement home, patients who arrived with vital signs absent, patients admitted directly to a service bypassing the ED, and patients transferred from other hospitals or without the appropriate advanced directive form were excluded. ED length of stay (LOS), number of investigations, type of imaging, disposition, and hospital LOS were recorded. Results: Eighty-seven patients were identified for enrolment; 55 were included. Mean (SD) age was 86.5 (8.8) years, and $20(36.4 \%)$ were male. Nineteen $(34.5 \%)$ patients had an advanced directive form indicating that they did not wish to be transported to hospital. Median (IQR) ED LOS was $6.8(5.2,8.6)$ hours for patients who did not wish to be transported, compared to $7.7(4.9,10.4)$ hours for patients with advanced directives indicating that they wanted transfer. Of the DNH patients, 12 (63.2\%) patients had laboratory investigations done while in the ED and 17 (89.5\%) patients had imaging. Of those with imaging, 8 patients had x-rays, 4 had CT scans, 4 had both $\mathrm{x}$-rays and CT scans, and 1 patient had an ultrasound. Of the DNH patients, $5(26.3 \%)$ were admitted to hospital with a median (IQR) hospital LOS of $38.2(28.3,122.0)$ hours. Conclusion: Transferring nursing home patients to the ED contrary to a DNH advance directive places a significant burden on both the patient and the health care system. Future studies could be done to assess factors that precipitate transfer to the ED despite DNH orders.

MP22 Leadership and administration competencies: establishment of a national consensus for emergency medicine

B. Thoma, MD, MA, J. Poitras, MD, FRCPC, CCMF(MU), R. Penciner, MD, MSc, CCFP(EM), FCFP, J. Sherbino, MD, MEd, B.R. Holroyd, MD, MBA, FRCPC, FACEP, R. Woods, MD, MMed, FRCPC; University of Saskatchewan

Keywords: administration, education, emergency medicine, leadership Introduction: The Royal College of Physicians and Surgeons of Canada (RCPSC) requires emergency medicine (EM) residency programs to meet training objectives relating to EM leadership and administration. However, there is no consensus on specific competencies that should be acquired. The purpose of this study is to establish a national consensus on the competencies for inclusion in an EM leadership and administration curriculum. Methods: A modified Delphi process was used to achieve consensus on appropriate competencies for inclusion in an EM administration and leadership curricula. An initial list of competencies was compiled by the authors using a search of the peer-reviewed and grey literature. The participants were 14 EM residency program directors and an additional 43 leadership and administration experts from across Canada who were recruited using a snowball technique. The Delphi process involved two iterative rounds of an electronic survey. The proposed competencies were organized using the CanMEDS 2005 Physician Competency Framework and presented in English or French. Consensus for the inclusion of a competency was defined a priori as $70 \%$ of participants agreeing on inclusion. Results: Thirty-five of 57 (61.4\%) participants completed round 1 and 30 of $35(85.7 \%)$ participants completed round 2 . Thirteen of 14 institutions with an FRCPC EM program had at least one participant complete both rounds. In round 1, the participants evaluated 93 competencies and suggested an additional 16. The aggregate results of round 1 were included in the round 2 survey to inform participant decisions. Ultimately, 59 of 109 (54.1\%) competencies achieved consensus for inclusion. Conclusion: Based on a national modified Delphi process, we describe 59 competencies, arranged by CanMEDS Roles, for inclusion in an EM leadership and administration curriculum. EM educators may wish to consider these competencies when designing local curriculum objectives to prepare residents for leadership or administrative roles.

$M P 23$ Is radial head subluxation associated with an increased radial metaphysis to capitellum distance in children aged 1 to 8 years? H. Gladwell, MD, CCFP(EM), S.L. McLeod, MSc, K. Selby, BSc, R. Lim, MD, S. McKillop, MD, N. Poonai, MSc, MD, FAAP, FRCPC; The University of Western Ontario

Keywords: emergency medicine, pediatrics, pulled elbow, radial head subluxation

Introduction: Radial head subluxation (RHS) is a common acute upper extremity injury in young children. The classic mechanism of 
traction on an extended arm is present in only 55 to $62 \%$ of cases. The objective of this study was to determine if there is a significantly greater radial metaphysis to capitellum distance on elbow x-ray in children with RHS compared to those who have a soft tissue injury of the elbow. Methods: This was a blinded, case-control study of children aged 1 to 8 years who presented to an academic children's hospital emergency department (annual census 38,000) from 2004 to 2011 and had an elbow x-ray series performed. Two authors, blinded to the patient's final diagnosis, independently measured the radial metaphysis to capitellum distance. A blinded pediatric radiologist corroborated $20 \%$ of the measurements. Interrater agreement was measured by Pearson correlation coefficient $(r)$. Patients with a final diagnosis of RHS were compared to age- and sex-matched controls that had no history consistent with RHS. All patients with a known musculoskeletal condition, fracture, dislocation, transfer from another facility, or an unplanned return ED visit within 30 days were excluded. Results: A total of 156 patients, 82 with RHS and 74 age- and sex-matched controls, were included in the analysis. There was no significant difference in radial metaphysis to capitellum distance on anteroposterior (AP) $\mathrm{x}$ ray for children with RHS versus controls $(8.6 \mathrm{~mm}$ v. $8.4 \mathrm{~mm} ; \Delta$ $-0.2 \mathrm{~mm}, 95 \%$ CI: $-0.6 \mathrm{~mm}, 0.3 \mathrm{~mm}$ ). There was also no difference in radial metaphysis to capitellum distance on lateral $\mathrm{x}$-ray in children with RHS versus controls $(7.4 \mathrm{~mm}$ v. $7.0 \mathrm{~mm} ; \Delta-0.4 \mathrm{~mm}, 95 \% \mathrm{CI}$ : $-0.8 \mathrm{~mm}, 0.1 \mathrm{~mm}$ ). The interrater agreement between the authors and radiologist was $r=0.95$ and $r=0.97$ for the AP and lateral views, respectively. Conclusion: There was no significant difference in the radial metaphysis to capitellum distance on AP or lateral elbow X-rays in children with RHS compared to soft tissue injuries. This study supports the convention that RHS is primarily a clinical diagnosis and argues against obtaining a radiograph of the contralateral elbow in equivocal cases.

MP24 Feasibility of and patient satisfaction withf HIV point-ofcare testing in the emergency department

R. Stenstrom, MD, PhD, E. Grafstein, MD, I. Poureslami, PhD, F. Osati, PhD, F.X. Scheuermeyer, MD, D. Harris, MD, PhD, G. Hunte, $\mathrm{MD}, \mathrm{PhD}$, S. O'Donnell, MD, MSc, K. Rich, MD, MSc, K. Nemethy, MD, MSc; St. Paul's Hospital

Keywords: emergency medicine, feasibility, HIV diagnostic testing Introduction: Since 2006, on recommendation of the CDC, emergency departments (EDs) in the US have adopted HIV screening. Canada has not followed suit. Laboratory test results take up to 10 days, and concerns about patient acceptance have been raised. Pointof-care (POC) testing provides rapid results, and immediate follow-up is arranged for positive tests. We hypothesized that the acceptance rate and patient satisfaction with point-of-care antibody testing would be high. Methods: This was a prospective, IRB-approved cohort study performed at St. Paul's Hospital ED between August 2009 and January 2011. ED patients were enrolled using probabilistic sampling of times of day/night and days of the week based on patient volumes. Inclusion Criteria: Age 19 to 75 years and able to understand/provide informed consent. Exclusion Criteria: Intoxicated or in extremis. Subjects HIV antibody status was obtained using the Biolytical INSTI POC test. Subjects completed a questionnaire regarding HIV risk factors, knowledge, perceived HIV status, and satisfaction score with POC testing (0-10 Likert scale). Results: A total of 2,001 patients were approached for the study, and 1,403 agreed to participate $(70.1 \%$; 95\% CI $68-72$ ). Participants were similar to nonparticipants for age, gender, and CTAS level. The mean age of subjects was 43.3 years; $58.4 \%$ of subjects were male. Patient satisfaction with point-of-care HIV testing was high (mean satisfaction score 9.6/10; $\mathrm{SD}=0.91$ ). The median amount of time required to perform each test was 7.2 minutes $(\mathrm{IQR}=4.7-10.1$ minutes). Conclusion: A rapid POC test for HIV-1 and -2 antibody has a moderate acceptance rate among all comers to the ED. Patient satisfaction with POC testing is high and the test can be performed rapidly, and this approach is feasible in the ED.

MP25 A structured educational intervention effectively improves morbidity and mortality $(M \& M)$ rounds: the Ottawa $M \& M$ model E. Kwok, MD, MHA, FRCPC, J.R. Frank, MD, MA(Ed), FRCPC, A.A. Cwinn, MD, FRCPC, J.R. Worthington, MD, FRCPC, L.A. Calder, MD, MSc, FRCPC; Department of Emergency Medicine, University of Ottawa

Keywords: adverse event, emergency medicine, medical error, quality improvement

Introduction: Morbidity and mortality (M\&M) rounds are an opportunity for health care professionals to systematically identify cognitive or system issues that affect patient safety and quality of care. The objective of this study was to implement a structured M\&M rounds model through an innovative educational intervention and to evaluate its impact. Methods: This before and after study enrolled participants in the Department of Emergency Medicine and Division of Trauma at a tertiary care teaching hospital from 2011 to 2012. The Ottawa M\&M rounds model included a multipronged intervention: (1) training presenters on case selection and analysis; (2) engaging interprofessional and multidisciplinary members; (3) creating an administrative pathway for acting on identified issues; and (4) executing a dissemination plan for lessons learned. We assessed impact via (1) review of M\&M presentation content; (2) pre/post surveys of presenters and attendees; and (3) departmental policy audit. Our outcomes were (1) improvement in case selection and analysis; (2) change in perception of individual practice and departmental level impact; and (3) increased number of policy changes as a result of $M \& M$ rounds. We analyzed the data with descriptive statistics, chi-square tests, and $t$-tests. Results: We analyzed 37 case presentations and found statistically significant improvements postintervention in appropriate case selection $(p<0.0001)$ and recognition of system issues $(p=0.01)$. Of $187 / 328(57.0 \%)$ survey respondents, we found significant improvements in perceived impact on clinical practice at both individual $(p=0.02)$ and departmental levels $(p=0.0001)$, as well as overall M\&M rounds attendance $(p=$ $0.001)$. We detected a significant increase in the number of systemic policy changes postintervention. Conclusion: The Ottawa M\&M model is an effective educational intervention that could be readily applied to any hospital department seeking to enhance quality of care and patient safety.

MP26 Exploring the prevalence of adverse events in the treatment of acute migraine headaches in the emergency department B.H. Rowe, MD, MSc, CCFP(EM), D.M. Dryden, PhD, E. SumamoSchellenberg, MPH, B. Vandermeer, MSc, B. Friedman, MD, MSc, I. Colman, $\mathrm{PhD}$; University of Alberta

Keywords: emergency medicine, migraine, safety, systematic review Introduction: Despite the widespread use of a variety of agents as treatments for acute migraine headaches, debate continues regarding the relative safety of the different agents. One of the most distressing side effects for patients is akathisia. This systematic review was designed to compare the safety of parenteral interventions to treat migraine headaches in adults presenting to emergency departments (ED), with a particular focus on akathisia. Methods: We searched 10 databases to January 2012, conference proceedings, clinical trials registers, and reference lists. Two reviewers independently selected studies, assessed methodological quality, and graded the evidence. One reviewer extracted and a second verified data. Findings were 
qualitatively summarized using risks (incidence) and $95 \%$ confidence intervals for specific adverse effects. A mixed treatment comparison was conducted for akathisia. Results: Nine classes of drugs were investigated in 71 controlled trials. Risk of bias was low for $28 \%$ of trials, unclear for $61 \%$, and high for $11 \%$. Overall, short-term side effects were infrequent, minor, and self-limiting; serious adverse events were uncommon. The risk of sedation with metoclopramide or neuroleptics was $17 \%$. The most common side effects for triptans were skin or local reactions and sedation. For DHE, the most common side effects were skin or local reactions, sedation, digestive issues, nausea or vomiting, and chest symptoms. There were few side effects reported for NSAIDs or opioids. Based on the mixed treatment comparison, the odds of experiencing akathisia symptoms following administration of metoclopramide or neuroleptic agents was approximately 10 times greater than with placebo. Conclusion: The two most common and efficacious nonnarcotic treatments for acute migraine (metoclopramide and prochlorperazine) also result in akathisia. Treatment of acute migraine headache in the ED should consider both drug efficacy and safety.

MP27 Limits to sensemaking: communication patterns in an urban emergency department

G.S. Hunte, MD, PhD, J.J. Joyce, PhD, W.H. McKellin, PhD, S.B. Sheps, MD, MSc; St. Paul's Hospital

Keywords: communication, emergency medicine, patient safety Introduction: Communication, essential to clinical sensemaking and safety, is often limited and interrupted in an ED. We describe communication patterns in an urban ED and demonstrate limits to sensemaking and collaborative interaction. Methods: Sixteen emergency department health care professionals (including nurses, physicians, and emergency staff) at an inner-city tertiary care ED were observed over a 4 -week period using the communication observation method. Audio recordings were transcribed verbatim and coded for communication events and interactions. Results: Overall, 3,663 communication events were identified over 24 hours and 27 minutes of observed time, with a new event occurring on average every 24 seconds ( 2.5 events/minute, range across roles 2.04-3.26 events/minute). Communication events were brief (median duration 7.0 seconds, 95\% CI: 6.5 to 7.5 seconds) and often interrupted (1.0 event/minute, range across roles $0.67-1.64$ events/minute) and concurrent (54\% of events across all roles). Physicians spent a significantly greater proportion of observed time in communication activity $(89 \%)$ than other clinical providers or staff, using computer-mediated channels almost a third of the observed time. Physicians and trauma and triage nurses, in particular, were compelled to "gain" time by overlapping communication (talking and writing/typing at the same time) because of limited opportunity to adjust relative to communication load. In addition, averaging two-way communication over an hour demonstrates the scarce opportunity to share sensemaking about patient care between physicians and clinical nurses (approximately 1 minute per patient per shift). Conclusion: ED care providers need a way to meaningfully collaborate and make sense of what is going on to create a collective clinical response and provide safe care. Time for shared sensemaking is limited and must be optimized and protected. Strategies to reduce communication load and allow for greater task focus, such as limiting off-topic interruptions to safety information, reducing overhead pages, and streamlining documentation channels, are required.

MP28 Improving stroke and TIA care in the emergency department: results from a provincial stroke improvement collaborative D.R. Harris, MD, MHSc, PhD(c), N. Kamal, PEng, PhD (Cand.), P. Aikman, MHA, J. Marsden, MD, C. Krause, MS; Stroke Services B.C., Provincial Health Services Authority
Keywords: emergency medicine, knowledge translation, neurosciences, quality improvement

Introduction: Stroke and transient ischemic attack (TIA) are common disorders cared for by emergency physicians. Best practices exist for the management of these disorders; however, these are not always followed. This study sought to measure the impact of a coordinated, provincial, structured improvement collaborative on care for stroke and TIA patients within emergency departments (EDs) in British Columbia (BC). Methods: This study was a qualitative and quantitative process evaluation of a provincial improvement collaborative. The collaborative followed an Institute of Healthcare Improvement (IHI) methodology, consisting of five workshops, biweekly webinars, and improvement coaching and support, over a 10-month period, from September 2011 to June 2012. This evaluation examined process measures of success, such as self-reported improvement and adherence to certain clinical process measures (e.g., time to CT). Collaborative participants submitted monthly reports that quantified their improvement using the Self-Assessment Score, which is used in IHI collaborative methodology. Results: Seventeen multidisciplinary teams participated, representing $27 \%$ of all EDs in BC (29/108) and all five health authorities. Further, all but one of the province's tPA-enabled sites were represented. Ninety percent of teams (15/17) reported a median Self-Assessment Score greater than 4.5/5.0, indicating significant and sustainable improvements in stroke and TIA care. No teams dropped out of the collaborative. Teams reported a mean of 5.5 (SD 2.4) significant improvements within their ED. For example, shortened doorto-CT times, improved triage and training, and implementation of standardized swallowing screens were shown. This collaborative showed higher success rates of self-reported improvement (typically only $30 \%$ of teams achieve significant improvement) and lower rates of dropouts than the published literature. Conclusion: Using an IHIstructured collaborative format, a provincial stroke and TIA emergency department quality improvement initiative was able to achieve significant improvements in adherence to best practices. A formal outcome evaluation is planned to measure the impact on stroke incidence, disability, and mortality.

MP29 Using computer simulation modeling techniques to evaluate interventions for increasing patient flow in the emergency department D.W. Savage, PhD, D. Woolford, PhD, B. Weaver, MSc, D. Wood, MD; Northern Ontario School of Medicine

Keywords: computer simulation modeling, emergency medicine, patient flow

Introduction: Many emergency department (ED) interventions have been developed to increase patient flow and reduce wait time (WT) and length of stay (LOS). These interventions have been investigated using a variety of research methods and in a variety of ED locations throughout the world. Computer simulation modeling (CSM) is one technique that may be useful for comparing a set of interventions in a controlled, experimental environment. As well, CSMs can be rapidly developed and used to evaluate a variety of interventions without impacting patients, staff, or infrastructure in the real system. Methods: Using historical ED patient data, we developed parametric distribution models that describe patient arrival, laboratory processing, diagnostic imaging, specialist consultation, and hospital admission for acute care patients (i.e., primarily CTAS 1, 2, and 3). The distributions were then integrated into a CSM, which represents the major processes of triage, physician initial assessment, labs, imaging, consultation, physician reassessment, and admission. We then compared the current system to three interventions: (1) a modified physician shift work schedule, (2) a triage liaison physician, and (3) the combination of the two interventions. Patient WT and LOS were measured and compared. Results: Our preliminary results showed that the WTs estimated by the model 
were $246(\mathrm{SD}=49), 184(48), 129(15)$, and $128(16)$ minutes for the current system and three interventions, respectively. The total LOS showed a similar pattern for the current system and three interventions with times of 523 (51), 458 (15), 390 (17), and 387 (19) minutes. The reduction in LOS for the three interventions was $12 \%, 25 \%$, and $26 \%$. Conclusion: The model results provide evidence that the triage liaison physician will have the greatest impact on WT and LOS; however, a relatively simple change in shift start times could be beneficial as well. CSM is a valuable technique for comparing ED interventions and should be used by managers to improve decision making.

MP30 Differences in knowledge, attitudes, and beliefs regarding pain management between EPs and RNs in the emergency department

K. Nixon, BScN, G. Martin, RN, MN, ENC, M. McGowan, MHK, K. Gaunt, MHSc, ENC, G. Bandiera, MD, D. MacKinnon, MD, J. Spence, MD; St. Michael's Hospital

Keywords: emergency medicine, pain management, staff knowledge, survey

Introduction: Effective pain management has been shown to improve care and allow for timely investigation and management while maintaining the validity of clinical assessments. However, the literature suggests that patients are commonly undertreated for pain in the emergency department (ED). Methods: In an academic, inner-city, level 1 trauma centre (annual volume 72,000), a 45-question survey assessing staff nursing $(\mathrm{RN})$ and physician (EP) knowledge, attitudes, and beliefs about pain management was designed and piloted by an interprofessional team. Completion was over a 4 -week period prior to introducing an emergency early pain management protocol and educational sessions. Results: The overall response rate was high (EP $87.5 \%$, RN $84.7 \%$ ), with gender differences (EP 57\% male v. RN 85\% female) and years of practice $(1-5$ years EP $20.8 \%$ v. RN $44.3 \%$; $11-15$ years EP $20.8 \%$ v. RN $11.5 \%$; > 20 years EP $12.5 \%$ v. RN 18\%) noted between respondents. Differences in primary analgesic goal were found ("enough to function" EP $43 \%$ v. RN $21 \%, p<0.05$; "patient pain goal" EP $29 \%$ v. RN 36\%, $p<0.05$ ), and both identified patients as the most accurate judge of pain (EP 76\% v. RN 97\%). One hundred percent of EPs versus $67 \%$ RNs reported 10-point pain scale as reliable $(p<0.05)$. Pain management was an equal priority with clinical care to both (EP $86 \%$ v. RN 85\%). Staff estimated that 51 to $75 \%$ (EP $48 \% \mathrm{v}$. RN $46 \%$ ) of patients had satisfactory analgesia, while unsatisfactory relief was attributed to "inadequate orders" (38\% EP v. 75\% RN, $p<$ $0.05)$ or "inadequate administration" (62\% EP v. $20 \% \mathrm{RN}, p<0.05)$. There were similar perceived external barriers ("awaiting diagnostics," $71 \%$ EP v. $80 \%$ RN, $p<0.05$; "excessive legislation" $18 \%$ EP v. $4 \%$ $\mathrm{RN}, p<0.05)$. Groups disagreed regarding impact of volume, workload, and acuity as a barrier $(51 \% \mathrm{RN} \mathrm{v} .42 \% \mathrm{EP}$ strongly agree, $p<$ $0.05)$ or practice influence ( $38 \%$ EP v. $33 \% \mathrm{RN}, p<0.05)$. Both estimated 1 to $5 \%$ incidence of addiction due to opioid treatment (52\% EP v. $51 \% \mathrm{RN})$, and EPs $(52.4 \%)$ indicated that it did not change practice. High levels of agreement were recorded for seniors' underreporting pain $(100 \%$ EP v. $93.7 \% \mathrm{RN})$ and impact of religion/cultural beliefs influencing pain tolerance (95.2\% EP v. $80.3 \%$ RN agree). Conclusion: Our results suggest that there may be significant and important differences in beliefs between EPs and RNs regarding pain management delivery in the ED. These differences would be important to acknowledge when developing knowledge translation and implementation strategies to support a new emergency-based early pain management protocol.

MP31 ASSESSING ambulatory vital signs in the workup of pulmonary embolism by way of a standardized 3-minute walk test
Q. Amin, MD, J.J. Perry, MD, MSc, CCFP(EM), I.G. Stiell, MD, MSc, FRCPC, S. Mohapatara, MS4, M. Rodger, MD, MSc, FRCPC; University of Ottawa, Department of Emergency Medicine

Keywords: clinical decision making, emergency medicine, pulmonary embolism

Introduction: The diagnosis of pulmonary embolism (PE) can be quite difficult to make given its highly variable clinical presentation. These patients are often exposed to a myriad of clinical decision tools as well as variation in their interpretation. Our objective was to determine whether a decrease in oxygen saturation or increase in heart rate while ambulating could be used as objective measurements to help in the diagnosis of PE. Methods: This study was a single tertiary care centre prospective cohort that enrolled patients greater than 18 years of age with a suspected or confirmed PE. Patients were enrolled directly from the emergency department or associated thrombosis clinic. The primary outcome of interest was looking into the value of ambulatory oxygen saturation in predicating PE (PE was defined as any PE treated by thrombosis physicians following confirmatory diagnostic testing). Patients were asked to walk for a fixed period of 3 minutes. A study pulse oximeter was used to continually measure heart rate and oxygen saturation levels. Descriptive and univariate analyses were conducted to determine whether a decrease in ambulatory oxygen saturation ( $\geq$ $2 \%)$ and/or a heart rate ( $>90$ beats per minute $[\mathrm{bpm}]$ ) had any correlation in a patient with PE by way of a standardized walk test. Results: A total of 114 patients, including 30 with PE (26.3\%), were enrolled. Baseline characteristics included mean age of 52.7 (19 to 95), female gender $62.2 \%$, mean heart rate $80.1 \mathrm{bpm}$, mean oxygen saturation $97.5 \%$, mean respiration rate $17.9 / \mathrm{min}$. There were significant associations between $a \geq 2 \%$ absolute decrease in ambulatory oxygen saturation from baseline and an ambulatory heart rate $>90 \mathrm{bpm}$ in those patients with PE. The ambulatory heart rate demonstrated superiority with a sensitivity of $93.3 \%$ (95\% CI 78.7 to 98.2 ), a specificity of $27.4 \%$ (95\% CI 19.0 to 37.8 ), and a negative likelihood ratio of 0.3 ( $95 \%$ CI 0.06 to 0.97 ). Change in ambulatory oxygen saturation had a sensitivity of $80.2 \%$ (95\% CI 62.7 to 90.5$)$ and a specificity of $39.3 \%$ (95\% CI 29.5 to 50.0 ). Conclusion: Our study showed that an ambulatory heart rate measurement $>90 \mathrm{bpm}$ and a $\geq 2 \%$ absolute decrease in ambulatory oxygen saturation from baseline are both objective measurements in the workup of PE. These variables may help in the diagnosis of PE when used in conjunction with a standardized 3-minute walk test.

\section{MP32 AN EPIDEMIOLOGIC profile of resuscitation systems of care performance in out-of-hospital cardiac arrest}

Z. Dewar, BSc, A. Travers, MSc, MD, J.L. Jensen, ACP MAHSR, A. Carter, MD; Emergency Health Services Nova Scotia

Keywords: emergency medicine, EMS, resuscitation

Introduction: Our aim was to link structure, process, system, and outcome resuscitation measures from community, emergency medical services (EMS), and in-hospital systems of care (SOC) to identify factors significantly associated with positive outcomes by examining the SOC as a whole. Methods: Data were collected from first responder charts, EMS dispatch, electronic patient care records, and hospital charts for all 2011 out-of-hospital cardiac arrests (OOHCA). Data were linked using deterministic linkage. Descriptive and chi-square analyses were performed on cases of presumed cardiac etiology. The primary outcome was survival to discharge. Results: EMS responded to 1,514 cases of possible OOHCA in 2011. Excluded cases included 189 $(12.5 \%)$ overtriage, no patient, missing data, duplicate entries, in-hospital cases, and interfacility transfers; 68 (4.5\%) traumatic OOHCA, 714 (47\%) no attempted resuscitation, and $27(1.8 \%)$ noncardiac etiology. A total of 516/1,514 (34\%) cardiac etiology cases were analyzed. 
Mean age was 66 years $(\mathrm{SD}=17.5)$, and 353 were male $(68.4 \%)$. In $222 / 516(43 \%)$, resuscitation was terminated in the field. A total of 294/516 (57\%) were transported; $122 / 516$ had sustained return of spontaneous circulation (ROSC) $(23.6 \%)$. Of cases with sustained ROSC, 42/122 (34.4\%) survived to discharge with a median cerebral performance category of 1 (90th percentile: 2 ). Factors significantly associated with survival include witnessed OOHCA $(p=0.005)$, first responder CPR $(p=0.008)$, epinephrine $(p<0.001)$, intubation $(p=$ $0.001)$, sustained ROSC $(p<0.001)$, ED TTH $(p<0.001)$, transfer to higher-level care $(p<0.001)$, and PCI $(p<0.001)$. Limitations include collection from administrative databases, limited first responder data, and limited availability of postarrest measures. Conclusion: To conclude, factors from each SOC were associated with survival. These findings have implications for prospective research, clinical practice, and policy to integrate SOCs to improve OOHCA outcomes.

MP33 Point-of-care ultrasound (ACES protocol) improves diagnostic accuracy and confidence in simulated cardiorespiratory scenarios

A. Parks, Medical Student, P.R. Atkinson, MD FCEM, G. Verheul, MD; Dalhousie Medicine, New Brunswick

Keywords: emergency medicine, medical education, simulation Introduction: Goal-directed point-of-care ultrasound (PoCUS) protocols have been developed to provide a structured approach to improving the diagnostic accuracy of the initial clinical assessment of the critically ill patient. We assessed the diagnostic impact of the addition of a simulated Abdominal and Cardiothoracic Evaluation by Sonography (ACES) protocol into simulated emergency medical scenarios. Methods: Following a PoCUS training program involving reading, videos, and an interactive workshop, we tested the diagnostic accuracy of 12 medical learners (clerks and residents) participating in standardized scenarios using a high-fidelity mannequin simulator (SimMan 3G) with a high-fidelity ultrasound simulator (CAE Vimedix). Participants were assessed during 72 simulated emergency cardiorespiratory scenarios using the two simulators. Differential diagnoses were collected from participants before and after PoCUS simulator incorporation in each scenario, and confidence surveys were completed. Data were analyzed using GraphPad Prism. Results: The addition of a goal-directed PoCUS protocol led to an improvement in differential diagnoses. Prior to use of PoCUS, $45(62.5 \%)$ correct primary diagnoses were made during the simulated scenarios compared to $64(88.9 \%)$ correct diagnoses following PoCUS (chi-square $14,1 \mathrm{df}, p=0.0002$ ). Goaldirected PoCUS was also shown to increase participants' confidence in their diagnoses. The mean confidence in diagnosis score pre-PoCUS was $52.2(\mathrm{SD}=14.7)$, while the mean post-PoCUS score was 81.7 (SD $=9.5)$. The estimated difference in means $(-28.36)$ was significant $(t=$ -7.71, $p<0.0001)$. Using PoCUS, participants were further able to narrow their differentials. The median number of diagnoses for each patient pre-PoCUS was $3.5(\mathrm{IQR}=3.0,3.8)$ with a median of $2.3(\mathrm{IQR}$ $=1.5,2.9)$ diagnoses post-PoCUS. The difference was significant $(\mathrm{W}=$ $0, p<0.001)$. Conclusion: We have demonstrated that the addition of a PoCUS protocol (ACES) to standard clinical assessment improves diagnostic accuracy and confidence in simulated cardiorespiratory scenarios. This is in keeping with clinical studies and supports the use of ultrasound during medical simulation.

MP34 A prospective evaluation of the utility of the ambulance call record to change the management of patient care in the emergency department

N. Cram, MD, M. Davis, MD, FRCPC, S.L. McLeod, MSc, M. Klingel, MSc, A. Dukelow, MD, FRCPC, M. Lewell, MD, FRCPC; The University of Western Ontario
Keywords: ambulance call report, documentation, emergency medicine, prehospital

Introduction: The ambulance call report (ACR) is used to record clinical history and physical examination findings, vital signs, prehospital medical treatments, and scene details. Important information is lost during verbal handover between paramedics and emergency department (ED) staff. The primary objective of this study was to determine how often the ACR is available to the ED physician at initial assessment and to determine if the ACR contains information that could change the ED management of patients. Methods: This was a prospective cohort study of adult patients arriving at one of two EDs at a tertiary care centre (annual census 125,000) by ambulance. At this centre, electronic ACRs are faxed to the ED upon completion and added to the patient's chart by ED staff. Physicians were asked to complete a data collection form for each patient regarding ACR availability and the perceived value of the ACR. Results: A total of 545 forms were collected over a 4-week study period. The ACR was available at first physician assessment for $82(15.0 \%)$ patients and available at some point during patients' ED stay for $154(28.3 \%)$ patients. Of patients who had an ACR available $(n=154)$, physicians reported that information changed or altered their treatment plan in $52(33.8 \%)$ cases. When an ACR was not available, $66.1 \%$ of physicians reported that the ACR would have provided valuable information, such as patient history (70.6\%) and vital signs (69.8\%). In $263(48.3 \%)$ cases, the physician received neither verbal handover from a nurse or paramedic nor an ACR. There were only 38 (7.0\%) cases where the physician received both verbal handover and an ACR was available. Conclusion: Although the ACR contains clinically relevant information that may change or influence ED management, physicians often assess, treat, and disposition patients without receiving the ACR.

MP35 Point-of-care ultrasound usage patterns following implementation of an archiving and quality assurance process in an academic tertiary care emergency department

C. Byrne, BSc, S.L. McLeod, MSc, D. Thompson, MD, FRCPC, R. Arntfield, MD, FRCPC, RDMS; The University of Western Ontario

Keywords: archiving, emergency medicine, point-of-care ultrasound, quality assurance

Introduction: Point-of-care ultrasound (US) has become routine practice in emergency medicine (EM) and is a requirement of Canadian EM training. Digital archiving and quality assurance (QA), common in many American US programs, are rare in Canadian emergency departments (EDs). Implementing a successful archiving and QA program requires additional effort by front-line staff, raising concerns for compliance and feasibility in a busy ED. The objective of this study was to evaluate US usage patterns of emergency physicians (EPs) following implementation of an archiving and QA process in an academic tertiary care ED. Methods: A review of an academic database of archived point-of-care US studies from two EDs of an academic tertiary care centre (annual census 125,000) was performed. The first 12 months after initiation of an obligatory archiving program was reviewed. Studies were stratified according to one of five examination types: abdomen, aorta, pelvis, cardiac (subxiphoid), and advanced applications (consisting of many examination subtypes). Results: Between January and December 2012, 7,720 point-of-care US studies were archived by 90 different EPs. Of these, $39.0 \%$ were training studies by EPs seeking US competency. The number of archived studies increased nearly threefold from the first quarter (January to March, 990 studies) to the fourth quarter (October to December, 2,837 studies); $80.4 \%$ of studies were generated from the four fundamental views: abdomen $(23.9 \%)$, aorta $(22.2 \%)$, pelvis $(20.4 \%)$, and subxiphoid cardiac $(13.8 \%)$. The remaining examinations $(19.6 \%)$ were "advanced" applications including cardiac $(54.0 \%)$, biliary $(14.7 \%)$, and thoracic 
(14.0\%). Conclusion: The large number of overall studies and compliance across many EPs in our 1-year experience support the feasibility of US archiving in a Canadian context with corresponding opportunity for QA and enriched US education and research.

MP36 An evaluation of the environmental factors and behavioural factors contributing to bike fatalities in Alberta

L. Lovstrom, BSc, MSc (Candidate), K.J. Webb, MLS, N. Ruest, MSc, C. Villa-Roel, MD, PhD (Candidate), A. Nettel-Aguirre, PhD, B. Hagel, PhD, B.H. Rowe, MD, MSc, CCFP(EM); University of Alberta

Keywords: bicycles, emergency medicine, fatalities, injuries Introduction: Bicycling is a popular recreational activity and an increasingly common transportation option; however, injuries can result in health professional visits, ED presentations, hospitalization, and death. Fatalities related to cycling have rarely been examined in detail in Canada and never in Alberta, which has two major urban centres and a large number of smaller communities. Methods: A retrospective chart review was conducted using coroner's charts for fatality cases with bicycle involvement that occurred between 1998 and 2011. Charts were identified by the medical examiner's offices in Calgary and Edmonton in conjunction with a prospective study of cycling crashes in both cities. Charts were reviewed and data were collected by trained research staff using standardized data collection forms. Descriptive statistics are provided as medians with interquartile ranges (IQR) and proportions with $95 \%$ confidence intervals (CI); helmeted and nonhelmeted subgroups were assessed based on police and hospital records and groups compared using chi-square or $t$-tests statistics, as appropriate. Results: Overall, 106 fatality charts for the 12-year period were identified and reviewed. Five cases were excluded from the analysis due to lack of information. Overall, 88 $(87 \%)$ were males; the median age was 47 (IQR 25, 58). The median time of event was 2:30 pm (IQR: $10 \mathrm{am}, 6 \mathrm{pm}$ ), with an even distribution of events throughout the week. The majority of events occurred during the summer months and peaked in September (22 [22\%]). Fatalities varied annually with a low of $3(1998)$ and a high of 12 (2007). More deaths occurred in urban (64 [63\%]) than rural locations, and gravel was the most common surface of cycling deaths $(77[76 \%])$. Collisions with motor vehicles occurred in 67 $(66 \%)$ cases, while being thrown from the bicycle resulted in 15 (15\%) deaths. Only $26(26 \%)$ cyclists were wearing helmets at the time of the event. Overall, 25 cyclists were known to be consuming alcohol at the time of the event; 26 deaths occurred at times of poor lighting, and only 5 (5\%) occurred during foul weather. Conclusion: Fatal bicycle crashes in Alberta remain infrequent and involve adults riding on roads and colliding with motor vehicles. In addition to helmet legislation for all age groups, deaths may be prevented by separation of cyclists from motor vehicles, increasing visibility aids in nondaylight hours, and avoidance of substance abuse while operating bicycles.

MP37 Sources of support for paramedics managing work-related stress in a Canadian emergency medical service

P. Bradford, MD, E. Donnelly, PhD, M. Davis, MD, FRCPC, A. Dukelow, MD, FRCPC, S. Mal, MD, S.L. McLeod, MSc, M. Lewell, MD, FRCPC; The University of Western Ontario

Keywords: emergency medicine, EMS, paramedics, stress Introduction: Paramedics are routinely exposed to work-related stress and may seek help from a variety of sources to mitigate the effects of stress on their health, family life, and ability to work safely and effectively. Greater clarity is needed in understanding how paramedics would seek help in anticipation of providing and funding needed resources in treating work-related stress. The objective of this study was to determine how paramedics access different types of support in a Canadian EMS. Methods: Paramedics in a municipal-based service in southwest Ontario (annual call volume 80,000) were invited to complete a 167 -item online questionnaire. Participants were asked to report the likelihood of seeking help from a supervisor, a partner or other coworker, a union representative, a base hospital educator, family, or a therapist. Participants were also asked about levels of occupational and organizational work-related stress. Repeated measures ANOVA and ANOVA strategies were utilized to analyze the data. Results: A total of 145 paramedics (a 54\% response rate) completed the questionnaire. Respondents were more likely ( $p<0.001$ ) to seek support from family or a paramedic work partner than from any other source. Respondents were less likely to seek support from (sequentially) a coworker, a therapist, a union representative, a supervisor, or a base hospital educator. As access to employee assistance services for respondents is administered by the supervisory structure, stress levels were analyzed for individuals who were more or less likely to seek support from their supervisor. Analysis revealed higher levels of organizational $(p<0.001)$ and operational $(p<0.05)$ workplace stress in those individuals who were unlikely to seek support from their supervisor. Conclusion: These findings indicate that investments in awareness and education on accessing help for paramedics suffering from work-related stress should be spent on peer programs and open houses with family and friends rather than sending supervisors for extra training in stress identification. Thought should be given to create confidential access to employee assistance programs for work-related stress issues to promote easier, nonthreatening access. Further work with paramedics needs to be done to remove the cultural stigma involved in coming forward to seek assistance.

\section{MP38 Nurse practitioner follow-up for patients who leave the emergency department without being seen}

N.M. Kelly, MN-NP; Queen Elizabeth II Health Sciences Centre

Keywords: emergency medicine, nurse practitioner

Introduction: Patients who come to the emergency department (ED) for care and leave without being seen (LWBS) by a health care provider are a significant concern for overcrowded departments. Follow-up for these patients provides an opportunity to ensure that appropriate medical care has been sought. The purpose of this study was to determine characteristics of patients who LWBS, such as acuity level, reason for leaving, and whether or not follow-up care was obtained. Methods: This was a prospective cohort study involving nurse practitioner telephone follow-up for all patients who LWBS from the Queen Elizabeth II Health Sciences Centre ED in Halifax, NS, over a 1-month period. Three attempts were made to contact the patient before efforts were abandoned. If the NP contacted the patient, a brief telephone interview was conducted. Results: A total of 207 of 4,913 total patients (4.2\%) LWBS. The median age was 35.67 years, and $60.9 \%$ were female. The average wait time was 179.7 minutes before patients LWBS, and $75 \%$ left the waiting room prior to registered nurse assessment. The majority of patients ( $n=117$ or $56.8 \%$ ) were triaged as urgent (CTAS 3 ). Ninety-four (45\%) patients were successfully contacted and interviewed within 1 week of leaving; $64 \%$ cited long wait times as their reason for leaving, and $60.6 \%$ followed-up with their family doctor (FD) or specialist. Conclusion: Lengthy ED wait times contribute to patients LWBS. Many of these patients seek medical attention elsewhere (FD, other ED, walk-in clinic, etc.). A follow-up process for patients who are considered "high risk" may be warranted to mitigate potential adverse health outcomes. Additional research will identify which patients should be considered high risk, for whom a follow-up process is indicated. 
MP39 Are there knowledge gaps in the diagnosis and management of patients with imported diseases presenting to Canadian emergency departments, and are those gaps being met? Needs assessment

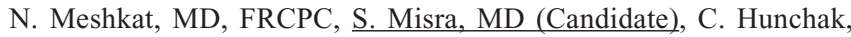
MD, CCFP(EM), P. Cleiman, CCFP(EM), Y. Khan, MD, FRCPC, L. Puchalski Ritchie, MD, FRCPC; University of Toronto

Keywords: education, emergency medicine, imported and tropical diseases

Introduction: The morbidity and mortality associated with "imported" diseases presenting to Canadian emergency departments is high. We conducted a needs assessment to assess knowledge gaps in the management of imported diseases by Canadian emergency physicians (EPs) and to identify available continuing medical education (CME) resources in this domain. Methods: A literature review was conducted using MEDLINE and EMBASE databases to summarize the challenges in the management of commonly encountered imported diseases. Following this, an anonymous online survey was administered to EPs across Canada using the CAEP survey deployment service from July to August in 2012. The survey identified self-reported gaps in knowledge and assessed knowledge using case-based vignettes. Finally, a list of CME resource titles was generated from a review of major academic emergency medicine (EM) journals, online cases, and conference topics from EM associations during 2010-2011. Two independent reviewers assessed the relevance of the resources; differences were resolved by consensus. Results: In total, 1,128 surveys were distributed and 296 participants were included in the study. The majority of participants had greater than 1 year of experience in EM. Most respondents reported no $(52.4 \%)$ or some $(45.9 \%)$ training in tropical medicine; $69.9 \%$ rated their comfort managing patients with imported diseases as "low," and $11.1 \%$ of participants reported an imported disease being misdiagnosed or mismanaged, most of which were malaria. Perceived need for further training was high. Conference workshops were the most highly requested CME modality, followed by case studies and podcasts. Correct answers to case vignettes ranged from 30.7 to $58.4 \%$. A total of 2,038 CME titles were extracted, and only 42 items were found to be relevant. Conclusion: Most Canadian EPs have minimal training in imported diseases, perceive a low comfort level in managing such cases, and identify a high need for CME opportunities; however, few specific educational resources are currently available.

MP40 What happens to children with suspected appendicitis when the appendix cannot be visualized on ultrasound? M. Ross, MD, G. Thompson, MD; University of Calgary

Keywords: appendicitis, emergency medicine, ultrasound

Introduction: We sought to determine the emergency department (ED) disposition of children age 2-17 with suspected appendicitis when the appendix cannot be visualized on ultrasound. We also sought to determine the incidence of CT scans, surgical consultations, and return ED visits. We will also examine the diagnostic accuracy of secondary signs of inflammation as seen on ultrasound to diagnose appendicitis in this population. Methods: We performed a retrospective chart review of all charts where an ultrasound was performed to rule out appendicitis between Jan. 1, 2007, and Dec. 31, 2008. We included all patients aged 2-17 years old presenting to the Alberta Children's Hospital pediatric ED with suspected appendicitis who underwent an ultrasound assessment of the appendix in which the appendix could not be visualized. Data were collected on ED visit details, laboratory markers, additional imaging, surgical consultations, dispositions, and return visits to the ED. Results: Data have been collected on 1,315 charts, of which 508 (39\%) met inclusion criteria for our analysis. A total of 297 children (59\%) were dis- charged home. Of these, 52 returned to the ED and 1 had pathologically confirmed appendicitis. A total of 153 children (30\%) were admitted to the ward, 53 of whom eventually went to the OR. An additional 53 children $(10 \%)$ went directly to the OR. A total of 109 $(22 \%)$ children had an appendectomy, of whom 88 had confirmed appendicitis at the time of surgery. The negative appendectomy rate was therefore $19 \%$. Thirty-five children $(7 \%)$ had a CT scan and 33 (6\%) had a repeat ultrasound. Conclusion: Our study suggests that the majority of children with nonvisualized appendicitis are discharged home with an acceptable rate of missed appendicitis (1/297 $=0.3 \%)$. The current practice at our institution rarely employs CT scan as an adjuvant imaging investigation, thereby minimizing radiation exposure in a potentially vulnerable pediatric population. Of those admitted to the ward for further observation, about one-third end up having an appendectomy. There was a high rate of negative appendectomies in our population, suggesting a potential role for additional imaging in children who would otherwise go to the OR based on clinical judgment alone. Our results support the efficacy and safety of our current practice patterns.

MP41 Knowledge translation of guidelines for the emergency department management of recent-onset atrial fibrillation and flutter: a retrospective chart review

A. Finningley, BScPT, R. Sadoway, R. Woods, MD, MMed, FRCPC; University of Saskatchewan

Keywords: atrial fibrillation, emergency medicine, knowledge translation

Introduction: Recent-onset atrial fibrillation and flutter (RAFF) is a common cardiac rhythm disorder managed by emergency physicians. Guidelines for the optimal emergency department (ED) management of symptomatic RAFF were released in 2010. Methods: A retrospective chart review was conducted to determine if management at a tertiary care ED in Saskatoon, SK, was influenced by the guidelines. A total of 1,076 charts were reviewed. Charts with symptomatic RAFF with a known onset of 48 hours and less were included for analysis. Two time frames were compared, prior to the release of RAFF guidelines (April 1, 2009, to March 31, 2010, $n=196$ ) and after educational dissemination of the guidelines (September 1, 2011, to May 31, 2012, $n=137)$. Results: There were similar baseline characteristics between the two groups, with the exception of significant increases in median duration of palpations and prevalence of several concurrent medical conditions in the postguideline group. There were no statistical differences between the two groups for rates of hospital admission $(27.5 \%$ v. $32.1 \%, p=0.36)$ and the use of electrical cardioversion $(24.6 \%$ v. $26.3 \%, p=0.73)$. Significant decreases were found in the postguideline group for median ED length of stay (LOS) (6.05 v. 5.27 hours, $p=0.03$ ) and consultation with cardiology $(55.7 \%$ v. $43.1 \%, p=0.02)$. Significant increases were observed regarding the use of rate control medications $(54.6 \%$ v. $67.2 \%, p=$ $0.02)$ and procainamide ( $2.6 \%$ v. $15.3 \%, p=<0.0001)$. Conclusion: Significant decreases in ED cardiology consults, ED LOS, and an increase in treatment with IV procainamide are changes in the postguideline phase that are consistent with the RAFF guidelines. However, a $32.1 \%$ hospital admission rate for RAFF and a significant increase in management with rate-controlling medication suggest that the guidelines are not being consistently applied in this ED.

MP42 Old school biomarkers in acute decompensated heart failure: serum sodium and hospitalization rates in emergency department patients

R. Stenstrom, MD, PhD, E. Grafstein, MD, F.X. Scheuermeyer, MD, D. Harris, MD PhD, G. Hunte, MD, PhD; St. Paul's Hospital 
Keywords: acute decompensated heart failure, emergency medicine, serum sodium

Introduction: Serum sodium $(\mathrm{Na}+)$ qualifies as a biomarker in patients with acute decompensated heart failure (ADHF). Elevated serum sodium has not been well studied in emergency department (ED) patients with ADHF. We hypothesized that patients with elevated serum sodium would be more likely to be admitted to hospital than patients with normal or low serum sodium. Methods: Our objective was to examine admission rates to hospital for ED patients with ADHF based on serum sodium levels while controlling for other serum electrolyte levels, renal function, and hemoglobin levels. This was a retrospective cohort, IRB-approved study in an urban tertiary care centre with an active cardiac program. From January 2006 to December 2008, demographic data, serum electrolyte levels, renal function, hemoglobin levels, and admission to hospital on that visit were recorded for all patients with a discharge diagnosis of heart failure. Based on serum sodium, each patient was categorized as one of hyponatremic $(\mathrm{Na}+<$ $135 \mathrm{mmol} / \mathrm{L})$, hypernatremic $(\mathrm{Na}+>145 \mathrm{mmol} / \mathrm{L})$, or normonatremic $(\mathrm{Na}+135-145 \mathrm{mmol} / \mathrm{L})$. Results: A total of 1,257 patients had an ED diagnosis of $\mathrm{ADHF} / \mathrm{CHF}$, and $73.7 \%$ were admitted; $58.4 \%$ of subjects were male, and the median age was 74.8 years (IQR $62-87$ years); and $77.5 \%$ of subjects were normonatremic, $16.7 \%$ were hyponatremic, and $5.8 \%$ were hypernatremic. The univariable odds ratio (OR) for admission in hypernatremic subjects (normonatremic as referent) was $2.49(95 \% \mathrm{CI}=1.26-4.93)$ and 1.51 for hyponatremic subjects $(95 \%$ $\mathrm{CI}=1.11-2.14)$. Using multivariable logistic regression to adjust for age, renal function, anemia, and potassium level, hypernatremia remained as an independent risk factor for hospital admission $(\mathrm{OR}=$ $2.61 ; 95 \% \mathrm{CI}=1.17-5.25)$. Conclusion: Hypernatremia is an independent risk factor for hospital admission in ADHF patients. It is unclear if altered sodium levels in ADHF patients are simply a marker for poor outcome, reflect another, related marker (arginine vasopressin), or directly contribute to the pathophysiology of ADHF.

MP43 Analysis of prehospital treatment of pain in the multisystem trauma patient at a community level 2 trauma centre

P. Bradford, MD, R. Bulatovic, MD, S. McKenzie, CHIM, E. Galbraith, BSc, D. Eby, MD, A. Dukelow, MD, FRCPC, M. Peddle, MD, FRCPC, S.L. McLeod, MSc, M. Lewell, MD, FRCPC, M. Davis, MD. FRCPC; The University of Western Ontario

Keywords: emergency medicine, EMS, pain, trauma

Introduction: The Ontario advanced life support patient care standards (ALS-PCS) limit the delivery of analgesia by advanced care paramedics (ACPs) to trauma patients who have suffered isolated extremity trauma. However, ACPs are able to establish online medical control to request analgesia for trauma patients who do not meet the ALS-PCS. The primary objective of this study was to determine how often analgesia is provided to trauma patients as defined by an injury severity score (ISS) $>12$ by either the ALS-PCS medical directive or through online medical control. Secondary outcomes included the proportion of patients who were transported by ACPs versus primary care paramedics (PCPs) and the time saved if analgesia was delivered in the field versus in the emergency department (ED). Methods: A retrospective chart review of trauma patients transported to a level 2 trauma centre from April 1, 2010, to March 31, 2011, was performed. Interfacility transports, walk-ins, and patients who fell in hospital were excluded. Cases were reviewed by a trained ACP auditor, medical student, and emergency medical services (EMS) trauma team leader physician. Results: A total of 228 patients with an ISS $>12$ were reviewed. Seventyeight were excluded (53 interfacility transports, 23 walk-ins, and 2 inhospital falls). Of the remaining 150 cases, 62 cases (41\%) had an ACP response where the potential to provide analgesia existed. Of these 62 cases, only $5(8.1 \%)$ received prehospital analgesia. The median (IQR) time to prehospital analgesia was $29(19,29)$ minutes. No patients were covered by the ALS-PCS medical directive, and all 5 patients who received analgesia required online medical control. Of the 57 ACP patients who did not receive prehospital analgesia, 37 (64.9\%) were given analgesia in the ED (median [IQR] of 68 [48, 165] minutes). Conclusion: Despite demonstrated rapid delivery, the frequency of prehospital analgesia use for multisystem trauma patients is extremely low. The majority are attended by a PCP who cannot administer analgesia. Promoting more frequent use of online medical control by an ACP may allow patients to receive analgesia much sooner. Consideration should be given to expanding prehospital directives for all paramedics to include pain control for multisystem trauma patients.

MP44 A before-after study to evaluate the effectiveness and usefulness of prehospital noninvasive ventilation in an urban setting A. Willmore, MD, R. Dionne, MD, CCFP(EM), J. Maloney, MD, FRCP, CCFP(EM), E. Ouston, ACP, I.G. Stiell, MD, MSc, FRCPC; Regional Paramedic Program for Eastern Ontario

Keywords: CPAP, emergency medicine, EMS, noninvasive Introduction: Noninvasive ventilation (NIV) is commonly used in the treatment of acute decompensated heart failure (CHF) and respiratory failure due to exacerbations of chronic obstructive pulmonary disease (COPD). In-hospital evidence is robust: NIV has been shown to improve respiratory status and reduce intubation rates. There is less evidence on prehospital NIV, although EMS adoption of this modality is increasing. The objectives of this study were (1) to measure the impact of prehospital NIV on morbidity, mortality, and transport times and (2) to audit the selection of patients by medics for appropriateness and safety. Methods: We conducted a before-after study from August 1 to October 31 in 2010 and 2011, before and after the implementation of prehospital NIV in a city of 1 million people with large rural areas. Medics were trained to apply NIV to patients with respiratory distress and a presumed diagnosis of CHF or COPD. Charts were selected using the search criteria of chief complaint of shortness of breath, emergent transport to hospital, and any patients receiving NIV in the field. Data extracted from ambulance call reports and hospital records were analyzed with appropriate univariate statistics. Results: We enrolled 373 patients, 186 in the pre-NIV group and 187 in the postNIV group, with a mean age of 71.5 years, female $51.4 \%$, and final diagnoses of CHF $18.9 \%$, COPD $21.9 \%$. Characteristics and transport times were similar between groups. In the post-NIV group, of 84 patients meeting NIV criteria, $41.6 \%$ actually received it; of 102 patients not meeting criteria, 5.2\% received NIV. There were 12 adverse events documented from a total of 36 NIV applications $(33.3 \%)$, all of them minor. Comparing the post-NIV to the pre-NIV groups, there were higher rates of ED NIV administration $(20.0 \% \mathrm{v}$. $13.4 \%, p<0.0001)$ and higher overall mortality $(18.8 \%$ v. $14.9 \%, p<$ $0.0001)$. There was no difference between groups in rates of ED intubation $(2.1$ v. $2.3 \%, p<0.001)$ or hospital length of stay $(6.8$ v. 8.7 days, $p=0.24)$. Conclusion: NIV was applied to a relatively small proportion of patients meeting criteria. No patient safety issues were identified. With respect to morbidity, mortality, and hospital length of stay, prehospital NIV failed to show benefit in this urban setting.

\section{MP45 Nerve blocks for initial pain management of pediatric} femur fractures: a systematic review

K.J. Black, MD, MSc, C.A. Bevan, MB BCh, N.G. Murphy, MD, J.J. Howard, MD; Dalhousie University

Keywords: child, emergency medicine, nerve block, pain Introduction: Pain relief for children with fractures is an essential part of emergency department (ED) management. Strategies include systemic analgesia and local nerve/compartment blocks. Our objective 
was to review the literature to assess benefits and harms of femoral nerve block (FNB) or fascia iliaca compartment block (FICB) compared to systemic analgesia for pain management in children with femur fractures in the ED. Methods: We systematically reviewed major bibliographic databases, clinical trial registries, conference proceedings and references of relevant studies. We included randomized controlled trials (RCTs) and quasi-RCTs assessing the effects of FNB or FICB for pain management in children $(<18$ years) with femur fractures. Two independent reviewers assessed study eligibility, completed data extraction, assessed risk of bias, and assessed study quality using the GRADE approach. Results: We identified 27 citations, and we included 3 studies. Only one study compared FICB and systemic analgesia. Two studies compared methods of administration of FNBs: one using ultrasound versus landmark guidance, the other using a continuous infusion versus a single injection. All three trials reported a significant reduction in pain at 15 to 30 minutes post-FNB/FICB. Heterogeneity of the studies prevented meta-analysis. High risk of bias and small sample sizes resulted in a low-quality rating for all outcomes. Conclusion: Low-quality evidence suggests that FICB improves pain relief and reduces adverse events compared to intravenous opioids for femur fractures in children. Overall, the benefits of FNB appear to outweigh the potential harms. Large, robust RCTs comparing systemic analgesia to FNB are needed to improve confidence in the results. The use of validated pain scores for future studies would help further strengthen the evidence base in this area.

MP46 An elder-friendly electronic hip fracture order set reduces the use of medications associated with delirium

J.J. Kim, BSc, MSc, T. Rich, MD, CCFP, K. Lonergan, BSc, D. Wang, $\mathrm{PhD}$, A. McRae, MD, E. Lang, MD; Alberta Health Services

Keywords: CPOE, delirium, emergency medicine, knowledge translation

Introduction: Delirium commonly develops among elderly patients admitted to the emergency department (ED) with hip fractures (Fx) Importantly, certain medications, including antiemetics, with anticholinergic and sedating properties as well as high-dose narcotics are associated with risk of delirium. We investigated whether a hip Fx order set (HFOS), designed to mitigate delirium risk and administered through computerized physician order entry, would improve how medications were prescribed and administered in the ED. Methods: We conducted a pre-post interventional cross-sectional study including three urban hospitals and examined a 10-month time frame before and after the March 22, 2011, HFOS implementation date. Eligibility consisted of patients $>60$ years admitted with an ICD-10 diagnosis of hip Fx. The new HFOS substituted ondansetron for dimenhydrinate as the antiemetic of choice and reduced narcotic dosing. The primary outcome was the proportion of patients for whom dimenhydrinate was ordered and administered as the initial antiemetic; secondary outcomes examined dosing and drug selections. Chi-square tests compared proportions, while Mann-Whitney nonparametric $U$ tests compared doses between study phases. Results: We analyzed 1,391 patients: 757 preHFOS and 634 post-HFOS, with a mean age of $82.3 \pm 9.2$ years and $72.6 \%$ female. Of patients who received an antiemetic medication (36.2\% pre; $30.6 \%$ post), the proportion of patients who received an initial dose of dimenhydrinate fell from $72.6 \%$ in pre-HFOS to $37.1 \%$ post-HFOS ( $p<0.0001$ ), with ondansetron rising in the intervention phase. The average first and overall total dose per patient receiving dimenhydrinate remained unchanged. With respect to narcotics, we observed a trend toward a reduced average first dose of fentanyl, hydromorphone, and morphine administered in the post-HFOS phase when compared to pre-HFOS. Conclusion: The creation of an elderfriendly HFOS designed to optimize antiemetic selection and temper narcotic analgesia was effective in reducing hip fracture patients' exposure to agents associated with delirium. Further work should explore the adequacy of pain control and confirm downstream benefits on patient outcomes.

\section{MP47 Identification of palliative care patients in the emergency department}

B. Tillmann, MD, S.L. McLeod, MSc, S. Baker, MD, MCFP, G. Joubert, MD, FRCPC, MHSc; The University of Western Ontario

Keywords: emergency medicine, palliative care

Introduction: Patients with life-limiting diseases commonly present to the emergency department (ED) during the course of their illness. The objective of this study was to determine the ability of ED physicians to accurately identify patients with diseases amenable to palliative care. Methods: Emergency physicians and residents working at an academic tertiary care centre (annual census 125,000) were invited to complete a 22-item, online questionnaire. Respondents were asked to identify if patients in 15 unique cases required emergent, outpatient, or no palliative care. The answers were compared to the answers provided by a palliative care provider (gold standard). The survey also included five questions designed to assess emergency physician comfort level in providing palliative care. Interrater agreement between emergency physicians and the gold standard in palliative patient identification was calculated. Results: Thirty-nine of $72(54.2 \%)$ participants completed the survey. Median (IQR) years of emergency medicine practice was 8 (4, $12)$, and $24(61.5 \%)$ respondents were male. Eight (21.0\%) respondents had formal palliative care training during residency. Of the 15 cases presented in the survey, in only 1 would the majority of respondents have referred the patient to palliative care. In contrast, the gold standard stated that in 11 cases, the patient would be appropriate for palliative care referral. The highest kappa value was 0.15 (95\% CI: 0 , 0.29 ). When asked if they felt the ED was an appropriate location from which to consult palliative care, the majority of respondents $(>75 \%)$, stated that it was. Twenty-six $(66.7 \%)$ respondents reported that they were comfortable providing palliative care. Conclusion: Although respondents stated that they were comfortable with providing palliative care and feel that this is part of their scope of practice, it appears that emergency physicians infrequently identify patients with diseases amenable to palliative care.

MP48 Evaluation of weight estimation and medication dosing errors during pediatric out-of-hospital cardiac arrest in a large urban/rural region

F. Bégin, MD, R. Dionne, MD, CCFP(EM), J. Maloney, MD, FRCP, CCFP(EM), J. Verdon, BSC; Regional Paramedic Program of Eastern Ontario

Keywords: emergency medical services, emergency medicine, errors, pediatrics

Introduction: Pediatric out-of-hospital cardiac arrest (POHCA) is an uncommon and very challenging situation for paramedics. Because of the infrequency of this scenario, the pediatric population is at risk of medication dosing errors related to weight estimation as well as calculation and preparation of nonstandard drug doses. Our objective was to determine the prevalence of weight estimation and medication dosing errors during POHCA. Methods: We conducted a health records review of all children 12 years old or younger with OHCA from January 2010 to December 2011 who were treated by ALS and BLS paramedics in the 9 EMS agencies in a large area of 10 counties and 5 cities. We defined a weight estimation error if the weight recorded exceeded the limits of the 3rd and the 97th percentile for the age according to the CDC growth charts and a medication dosing error as 
an arbitrary dosage variation exceeding $20 \%$ of the appropriate weightbased dose. We collected data from the patient care reports and used descriptive statistics with 95\% CIs. Results: We included 34 POHCA cases with a mean age of 31 months and $58.8 \%$ males. Of these, 13 (38.2\%; 95\% CI 23.8 to 55.1 ) had inappropriate weight estimation by ALS and BLS paramedics. Of the 10 patients who received epinephrine by ALS paramedics, 6 (60\%; 95\% CI 30.8 to 83.3$)$ had an inappropriate dose administered or a protocol violation. Conclusion: POHCA is a very rare event, and inappropriate weight estimation and drug administration by paramedics during POHCA are highly prevalent in our EMS setting. We identified a serious problem that mandates further training for the paramedics. The implementation of a validated tool to estimate pediatric patient weight and medication dosage should also be considered.

MP49 Pharmacist-led medication review in high-risk emergency department patients: a workload estimate

C.M. Hohl, MDCM, FRCP, MHSc, L.N. Reddekopp, Bachelor of Nursing, E. Yu, MS, R. Tsang, Doctorate of Pharmacology, N. Partovi, Doctorate of Pharmacology; University of British Columbia

Keywords: emergency medicine, medication review, patient safety, pharmacist

Introduction: Accreditation Canada, a nonprofit organization that accredits health care institutions based on the quality of care provided, recommends that health care providers conduct medication review on all patients at transitions in care such as those occurring in emergency departments (ED). Medication review includes obtaining a best possible medication history and reviewing the patient's medications to identify medication-related problems. Our objective was to provide a perpatient workload estimate for medication review in high-risk patients. Methods: This was a prospective observational substudy of a demonstration project conducted in three British Columbian EDs. Pharmacists conducted medication review on a systematically selected sample of patients who had been classified as high risk for adverse drug events using a clinical decision rule. Pharmacists were asked to focus their medication review on medications that may have contributed to symptoms leading to the ED visit and on those that might cause adverse events in the short term. A trained observer timed the pharmacists using standardized and piloted data collection forms during scheduled data collection shifts. We calculated median times with nonparametric confidence intervals (CI) using methods described by Hollander and Wolfe (1973). Results: We observed 14 clinical pharmacists conducting medication review on 58 high-risk patients. We observed a median of 4 interviews per pharmacist (IQR 3-5.5). Medication review took a median of 21.4 minutes (95\% CI: 19.4-26.7) per patient. The most time was spent on interviewing patients (6.5 minutes, 95\% CI 5.6-8.4), documentation (5.8 minutes, 95\% CI 5.4-7.6), and on reviewing the patient records prior to their assessment (5.4 minutes, 95\% CI 4.9-7.2). Conclusion: This pilot study allowed us to estimate the workload impact for pharmacists when reviewing medications of high-risk ED patients. Further research is necessary to determine whether ED-based medication review is associated with any health benefits and what the workload implications are for other health care professionals.

MP50 Effective intraprofessional ED consultations for collaboration, patient safety, and quality care: qualitative needs assessment A. Murnaghan, MD, J.R. Frank, MD, MA(Ed), FRCPC, R. Zemek, MD, FRCPC, K. Moreau, PhD; University of Ottawa, Department of Emergency Medicine

Keywords: consultations, education, emergency medicine, intraprofessional
Introduction: Effective consultation and intraprofessional collaboration are key domains of competence required for all physicians that impact patient safety, ED wait times, and length of stay. However, little literature exists on the characteristics of effective consultations, and there is no validated gold standard. This study was designed to identify enabling and impeding characteristics that were perceived to impact the effectiveness of ED consultations at two large teaching hospitals. Methods: We conducted a series of 1-hour focus group sessions to discuss specific characteristics that make an ED consultation effective and promote improved patient care as well as identifying characteristics that impede care with proposed solutions. Each focus group consisted of a mix of emergency department and off-service residents. The conversations were recorded, transcribed verbatim, and then coded into recurrent themes by two research team members. The overall process resulted in the development of thematic categories that describe the enabling and impeding characteristics of an effective emergency department consultation. Results: Data saturation was obtained after analysis of the first two transcripts and confirmed with a third session. Seven thematic categories emerged from the focus group sessions, including the appropriateness of consult, effective communication, patient treatment, professional conduct, reasoning for consult, required information, and timing of consult. Proposed recommendations for improved consultations included being knowledgeable of local admission policies, introducing yourself, asking a clear question, telling the truth, continuing to treat the patient until the consultant arrives, as well as respecting and helping the consultant if needed. Conclusion: This needs assessment study identified seven enabling and impeding characteristics of an effective emergency department consultation. A model of effective ED consultation can be developed using these elements that may improve disposition times, decrease time spent in the ED, enhance patient safety, and improve MD satisfaction with ED-consultant interactions.

MP51 Evidence to Excellence: a province-wide sepsis collaborative for harmonization of sepsis care

J. Marsden, MD, L. Newton, BAA, J. Cordeiro, BAA, L. Zibrik, MA, N. Kamal, PEng, PhD, (Cand.), K. Ho, MD; University of British Columbia

Keywords: emergency medicine, quality improvement, sepsis

Introduction: Evidence to Excellence (E2E) was created as a quality improvement initiative to improve emergency health care in the province of British Columbia. In 2009, E2E brought together emergency clinicians and front-line administrators from 19 hospital sites across the province through a sepsis collaborative. As part of this, a 3year (2009-2012) CIHR-funded grant was obtained to evaluate the ability of E2E to improve sepsis care in BC. Methods: Focus group, survey, and clinical outcome data were collected from each of the participating sites. The three clinical outcomes measured were (1) patients receive 1 litre fluid bolus within 90 minutes of admission; (2) antibiotics were given within 60 minutes of admission; and (3) blood cultures were collected before the antibiotics were administered. Statistical process control (SPC) charts were used to visually represent performance of and system variation within the collaborative over time. Additionally, data from patients who were deemed to have severe sepsis as indicated by an elevated initial lactate level were analyzed to determine if there was a difference in clinical outcomes between this group and the overall septic patient group. Results: Thirteen of 19 sites reported sufficient data on clinical outcomes. Collaborative-wide, substantive gains in achieving the three clinical outcome goals for sepsis patients were demonstrated. Furthermore, qualitative data helped build a picture of how the collaborative was influenced by quality improvement activities such as learning sessions and webinars. Conclusion: SPC charting was able to show improved outcomes and harmonization 
of care across many but not all of the sites. The qualitative data provided useful insights as to how and why these improvements were realized as well as identifying barriers to improvement.

MP52 Do learners in the emergency department help or hinder emergency physician productivity?

A. Dukelow, MD, FRCPC, D. Thompson, MD, FRCPC, C. Rhiger, RN, M. Klingel, MSc, S.L. McLeod, MSc; The University of Western Ontario

Keywords: emergency medicine, learners, productivity Introduction: In recent years, Canadian emergency department (ED) volumes and the number of medical trainees have increased. More learners are being placed in "teaching hospitals" and in busy community EDs. The impact of individual or multiple learners on emergency physician (EP) productivity is unknown. The primary objective of this study was to determine the impact of learners on EP productivity. The secondary objectives were to determine the impact of two learners, more than two learners, and a senior resident on EP productivity. Methods: This was a retrospective chart review of all patients arriving at one of two EDs at a tertiary care centre (annual census 125,000) between February 1-28, 2012, March 15-30, 2012, and June 1-30, 2012. The most responsible EP for each patient, number of learners scheduled on the EP's shift, and level of each learner were recorded. Learners were categorized as medical students, junior learners (postgraduate years 1-2), and senior learners (postgraduate years 3-5). Results: Fifty different EPs worked 1,119 shifts and saw 20,437 patients during the study period; $328(29.3 \%)$ shifts had no learners, $529(47.3 \%)$ shifts had one learner, 261 (23.3\%) shifts had 2 learners; and $1(<1 \%)$ shift had more than 2 learners scheduled. EPs saw a mean (SD) $2.3(0.7)$ patients per hour with no learners and $2.6(0.7)$ patients per hour with at least one learner on shift $(-\Delta 0.3,95 \% \mathrm{CI}$ : $0.4,-0.2)$. There was no difference in EP productivity when one learner was present on shift compared to two learners $(\Delta-0.02,95 \%$ CI: $-0.1,0.1) ; 161(14.4 \%)$ shifts had senior residents. EPs saw a mean (SD) 2.5 (0.7) patients per hour with no senior resident and $2.9(0.8)$ patients per hour when working with a senior $(-\Delta 0.4,95 \% \mathrm{CI}:-0.6$, 0.3). Conclusion: Clinical teaching is an essential part of emergency medical practice and the future of ED patient care. Despite the additional academic responsibilities, the presence of a learner improved EP productivity in this study.

MP53 Assessing emergency department quality of migraine care in an Ontario Local Health Integrated Network

W. Supapol, PhD, M. Ackerman, MD, A. Worster, MD; McMaster University

Keywords: emergency department, emergency medicine, migraine, opioids

Introduction: Opioids are not recommended as primary therapy for migraines due to the availability of more effective medications and the potential for opioid abuse/addiction among other reasons. We evaluated the prevalence of opioids as primary therapy for migraines at 12 Ontario emergency departments (EDs) within a single Local Health Integrated Network (LHIN). Methods: We reviewed approximately 100 randomly selected charts with a National Ambulatory Care Reporting System (NACRS) most responsible diagnosis (MRDX) coding of migraine at each ED for adult visits occurring between July 2009 and June 2011. Patients with cancer or headaches due to medical procedures were excluded. Our two trained data abstractors used standardized data collection procedures and forms, and data were simultaneously double abstracted and agreed upon for accuracy. All ED records were examined for primary opioid therapy by any route. We deter- mined the proportion of migraine visits where opioids were administered as primary therapy and $95 \%$ confidence intervals $(95 \% \mathrm{CI})$ and tested whether the observed proportion was significantly different from a hypothesized standard of 5\% using the binomial proportion test. Results: All 12 hospitals agreed to participate. The proportion of patients receiving primary opioid therapy for migraine ranged from $0 \%$ (95\% CI: $0-3.0)$ to $69 \%(59-77)$. At 6 hospitals, these proportions ranged from 0 to $8 \%$, which were not significantly different from the standard. At the other 6 hospitals, these proportions ranged from 24 to $69 \%$, which were significantly different from the standard. At 2 hospitals where $>60 \%$ of migraine patients received primary opioid therapy, repeat visits by a small number of patients were responsible for over $45 \%$ of migraine visits. Conclusion: Half of the hospitals in the LHIN met the standard for opioid primary therapy; $5 \%$ or less primary opioid therapy is an achievable goal. Opioid therapy is easy to measure and useful as a quality indicator for ED migraine care.

\section{MP54 Survival in neoplastic meningitis as a function of diagnostic modalities}

J. Shin, MD, S. Ahn, MD; Asan Medical Center, University of Ulsan, College of Medicine, Seoul, Korea

Keywords: emergency medicine, neoplastic meningitis, prognosis Introduction: Neoplastic meningitis (NM), also known as leptomeningeal carcinomatosis, is a common late-stage complication of cancer. It is diagnosed by the presence of malignant cells within cerebrospinal fluid (CSF) or brain and spinal cord imaging with magnetic resonance imaging (MRI). Patients with NM were divided into three diagnostic subcategories, and we tried to determine overall survival of $\mathrm{NM}$ as a function of diagnostic modalities. Methods: Between the years 2003 and 2010, 150 patients with solid tumors or lymphoma were newly diagnosed as NM at Asan Medical Center Emergency Department. All CSF examinations were performed in lumbar punctures, and three bottles of spinal fluid samples were gathered from each patient at a time. These patients were divided into three groups: positive for both cytology and MRI $(n=64)$, positive cytology with negative MRI $(n=43)$, and negative cytology with positive MRI $(n=43)$. Overall survival was measured in weeks, from the date of NM diagnosis until the date of death or last follow-up. Survival curves of these subcategories were plotted according to the Kaplan-Meier methods. Results: Overall median survival from NM was 8 weeks. CSF was positive for malignant cells in $107(71.3 \%)$, and MRI was positive in $107(71.3 \%)$ patients. Survival did not significantly vary among three groups (CSF [+], MRI [-]: median 12 weeks, range 1 to 80 [95\% CI $7-$ 17]; CSF [-], MRI [+]: median 12 weeks, range 1-66 [95\% CI 7-17]; CSF [+], MRI [+]: median 6 weeks, range 1-64 [95\% CI 3-9], $p=$ 0.306). Conclusion: When considering diagnostic modalities, CSF examination and MRI showed same sensitivities, and the survival of $\mathrm{NM}$ was similar in patients with different diagnostic modalities.

\section{MP55 Risk factors for serious underlying pathology in adult emergency department nontraumatic low back pain patients}

V. Thiruganasambandamoorthy, CCFP(EM), MSc, E. Turko, MD, D. Ansell, MSc, A. Vaidyanathan, MBBS, G.A. Wells, PhD, I.G. Stiell, MD, MSc, FRCPC; University of Ottawa, Department of Emergency Medicine

Keywords: emergency department, emergency medicine, low back pain, nontraumatic

Introduction: Nontraumatic low back pain (LBP) is a common ED presenting complaint and can be caused by serious pathologies that require immediate intervention or that lead to death. The primary goal of this study is to identify risk factors associated with serious pathol- 
ogy in adult nontraumatic ED LBP patients. Methods: We conducted a health records review and included patients 16 years or older with nontraumatic LBP presenting to an academic ED from November 2009 to January 2010. We excluded patients with confirmed nephrolithiasis and with typical renal colic presentation. We collected 56 predictor variables (demographic, historical, medication, clinical examination, investigation, and disposition) and outcomes within 30 days (compression fracture, osteomyelitis, spinal abscess, malignancy, cauda equina, disk prolapse requiring surgery, any condition requiring intervention, or death related to a cause of LBP). We determined outcomes by computerized tracking of patient records at the study hospital (only regional centre with vascular and neurosurgery). All outcomes were confirmed by a blinded reviewer. We used univariate analysis and recursive partitioning to identify the risk factors for serious pathology. Results: Among the 31,705 ED visits, 330 (1\%) were due to nontraumatic LBP. Data were available for 329 patients: mean age 49.3 years, females $50.8 \%$, previous cancer $6.1 \%$, osteoporosis $1.8 \%$, and on anticoagulants $4.0 \%$. ED imaging performed included $\mathrm{x}$-rays $17.3 \%$, CT $3.7 \%$, and MRI $4.0 \%$. Twenty-two $(6.7 \%)$ patients suffered outcomes (one death, five compression fractures, four malignancies, four disk prolapse requiring surgery, two retroperitoneal bleed, two osteomyelitis, one each of epidural abscess, cauda equina, and leaking abdominal aortic aneurysm graft). Risk factors for the above outcomes were use of anticoagulant medications (OR 15.6; 95\% CI 4.2, 58.5), decreased sensation on physical examination (OR 6.9; $95 \%$ CI 2.2 , 21.2), pain that is worse at night (OR 4.3; 95\% CI 0.9, 20.1), and pain that persists despite appropriate treatment (OR 2.2; 95\% CI 0.8, 5.6). These four predictors would identify LBP patients with serious pathology with $91 \%$ sensitivity (95\% CI 70-98) and 55\% specificity (95\% CI 54-56). Conclusion: We successfully identified risk factors associated with serious pathology among ED LBP patients. Future prospective studies are required to derive a robust clinical decision rule to identify those with serious underlying pathology.

MP56 Massive acetaminophen overdose treated with immediate hemodialysis: is the antidote removed faster than the toxin?

M.L. Sivilotti, MD, MSc, D.N. Juurlink, MD, PhD, L. Hanly, PhD (Cand), I. Lenga, MD, R. Poley, MD, J.S. Garland, MD, M. Thompson, MD; Ontario Poison Centre

Keywords: acetaminophen, acetylcysteine, emergency medicine, hemodialysis

Introduction: The role of hemodialysis for acetaminophen (APAP) overdose is debated. While APAP (molecular weight 151 daltons) is readily dialyzable, its antidote acetylcysteine (163 daltons) is as well. We occasionally recommend hemodialysis provided that it can be started immediately for the rare patient with features of so-called "mitochondrial paralysis" (lactic acidosis, altered mental status, hypothermia, hyperglycemia) following massive APAP overdose. Methods: A prospective case series, in which serum acetylcysteine concentrations during hemodialysis were also measured. Results: Three patients (ages 20 to 65 years) each presented comatose and acidemic several hours after ingesting $>1 \mathrm{~g} / \mathrm{kg}$ of APAP. Two were hypothermic (lowest $31.5^{\circ} \mathrm{C}$ rectal) and hyperglycemic. Serum lactate ranged from 7 to $12.5 \mathrm{mM}$. Each was intubated, and initial APAP concentrations were as high as $5,980 \mu \mathrm{M}(900 \mu \mathrm{g} / \mathrm{mL})$, an order of magnitude over the traditional threshold for antidotal treatment. Intravenous acetylcysteine was initiated between 10.8 and $\approx 18$ hours postingestion, and additional doses were empirically administered during hemodialysis to compensate for possible antidote removal. A single run of 3 to 4 hours of hemodialysis removed 10 to $20 \mathrm{~g}$ of APAP ( 48 to $80 \%$ of remaining body burden), reduced serum APAP concentrations by 56 to $84 \%$ (total clearance 3.4 to $7.8 \mathrm{~mL} / \mathrm{kg} / \mathrm{min}$ ), accelerated native APAP clearance (mean elimination half-life 580 minutes predialysis, 120 minutes during, and 340 minutes postdialysis), and extinguished the lactic acidosis. Total serum acetylcysteine concentrations ranged from 220 to $1,980 \mu \mathrm{M}$ during hemodialysis. Extraction ratios of acetylcysteine across the dialysis circuit ranged from 73 to $87 \%$, and the dialysance was 3.0 to $5.3 \mathrm{~mL} / \mathrm{kg} / \mathrm{min}$. All three patients recovered fully, with only one developing ALT > 1,000 IU/L, and none developing coagulopathy or other signs of liver failure. Conclusion: When massive APAP ingestion is accompanied by coma and lactic acidosis, immediate hemodialysis can result in rapid biochemical and clinical improvement. As expected, hemodialysis more than doubles the overall clearance of both APAP and acetylcysteine. Recognizing that acetylcysteine dosing is largely empirical, we recommend that the dosing of acetylcysteine be at least doubled whenever patients being treated with acetylcysteine undergo hemodialysis and that a half-load be administered after a dialysis session that exceeds 6 hours.

\section{MP57 National EMS survey on selective C-spine immobilization in the field}

B. Chaudry, MD, C. Vaillancourt, MD, MSc, FRCPC, CSPQ, M. Gatien, MD, M. Nolan, MA, ACP, D. Socha, BSc, PCP, P. Poirier, ACP ; University of Ottawa, Department of Emergency Medicine

Keywords: C-spine, decision rule, emergency medicine, EMS Introduction: Current $\mathrm{C}$-spine immobilization practices are not evidence based, and $<1 \%$ of patients boarded and collared have a $\mathrm{C}$-spine injury. The Canadian $\mathrm{C}$-spine rule (CCR) was validated by paramedics and allows for safe and selective immobilization of the C-spine in the field. We sought to determine the barriers and facilitators to the implementation of the CCR and its current use by Canadian paramedics. Methods: We conducted an online national survey among EMS decision makers belonging to Emergency Medical Services Chiefs of Canada. EMSCC counts 343 members (not all of whom are EMS chiefs) from every EMS organization in Canada. The provinces of BC, $\mathrm{AB}, \mathrm{NS}, \mathrm{NB}$, and PEI have a single EMS provider, whereas other provinces are served by a various number of EMS services. Survey questions were verified for internal validity and piloted before their distribution using a modified Dillman technique (multiple attempts). We assessed barriers and facilitators using a 5-point Likert scale and report descriptive statistics. Recruitment took place in April-June 2012. Results: The 50 respondents were deputy chiefs $28.6 \%$, medical directors $28.6 \%$, EMS chiefs $20.4 \%$, quality officers $12.2 \%$, and educators $4.1 \%$ from EMS services with a mean number of paramedics of 658 (range 10-4,500) and a mean population size of 893,000 (range 12,000-4.4 M). Among 29 responding EMS providers, 17 (58.6\%) already used selective immobilization, and $10(58.8 \%)$ used the CCR ( 7 used a locally developed protocol). Selective immobilization is used in all provinces except Manitoba. Among those surveyed who did not currently use selective immobilization, $100 \%$ intended to do so in the next 2 years. The main perceived barrier to CCR implementation is the practice standard set by provincial regulators (scored 3.2/5). The main facilitators to CCR implementation include support from EMS services (4.7/5), the perceived importance to decrease unnecessary immobilizations (4.7/5), and time efficacy (4.5/5). Most respondents $(54.0 \%)$ believed the CCR should miss $<2 \%$ of injuries, $28.0 \%$ would accept that it missed up to $5 \%$ of injuries, and $16.0 \%$ believed it should miss none. Conclusion: A growing number of Canadian EMS providers safely perform selective $\mathrm{C}$-spine immobilization in the field, many of them using the CCR. Provincial regulation is not perceived to be a significant barrier to CCR implementation, which is perceived to be of great benefit for patients and EMS providers. 


\section{Moderated Poster Presentations (Electronic Posters)}

\section{EP01 Motion artifact reduction of ECG signal allows for greater chest compression fraction during CPR}

S.M. Fernando, MSc, J.B. Baylis, BSc, A. Szulewski, MD, D.W Howes, MD; Departments of Emergency Medicine and Critical Care, Queen's University

Keywords: cardiac arrest, CPR, emergency medicine Introduction: Minimizing interruptions during CPR is associated with better outcomes. ECG rhythms cannot be analyzed during CPR because the compressions produce artifacts. One of the most common reasons for interruptions in chest compressions is for mandatory rhythm analysis prior to shock. Therefore, reducing the duration of this interruption may lead to better outcomes. The purpose of this study was to evaluate CPR performance using an alternate protocol that takes advantage of software that removes CPR artifact. Methods: We compared chest compression fraction (CCF) of second-year ICU residents running the same scenario using two different protocols. In the control, they were told to use the standard ACLS protocol. For the intervention, they ran a similar scenario, using a defibrillator with a filtered rhythm and a protocol that minimized rhythm checks. Results: Preliminary data show that using this new methodology seems to greatly increase time spent on the chest. CCF increased to $90.523 \%$ (95\% CI $=88.889$ 92.157) compared to the standard ACLS protocol, which produced a CCF of $74.457 \%(95 \% \mathrm{CI}=71.642-77.273 ; p<0.05)$. Conclusion: This novel technology may permit changes in resuscitation protocols that reduce CPR interruptions. Further research is warranted to evaluate the impact of the improved CPR on patient outcomes.

EP02 Outcomes of pediatric patients presenting to the emergency department with a medication-related visit

P.J. Zed, PharmD, K.J. Black, MD, MSc, E.A. Fitzpatrick, RN, MN, ENC(C), S. Ackroyd, PhD, N.G. Murphy, MD, J.A. Curran, PhD, N.J. MacKinnon, PhD, D. Sinclair, MD; University of British Columbia

Keywords: adverse drug events, emergency medicine, patient safety, pediatrics

Introduction: Outcomes among pediatric patients presenting to the emergency department (ED) for medication-related visits (MRV) are not known. Our objective was to determine short-term outcomes of pediatric patients presenting to the ED as a result of a MRV in a large tertiary care Canadian pediatric teaching hospital. Methods: A prospective, observational study of pediatric patients presenting to the ED over a 12-month period was conducted. Patients were randomly selected for inclusion using a systematic sampling methodology stratified a priori by time of day and day of week to ensure a generalizable sample. A pharmacist assessed subjects to determine the rate, characterization, severity, and preventability using predefined definitions. Patients were followed until ED/hospital discharge for short-term outcome, which included rate of admission and length of stay (LOS). The LOS for MRV versus non-MRV was compared using Mann-Whitney nonparametric test. Results: A total of 2,028 patients were enrolled (mean age $6.1 \pm$ 5.0 years, $52.6 \%$ female). A MRV was identified in 163 patients, $8.0 \%$ (95\% CI $7.0-9.3)$ of patients. Overall, $145(7.2 \%)$ of the study cohort was admitted to hospital. The rate of admission in patients with a MRV was $27.0 \%$ (95\% CI 20.4-34.5) compared to $5.4 \%$ (95\% CI $4.4-6.5$ ) among those without a MRV. The probability of admission was significantly higher among patients with a MRV (OR 6.5, 95\% CI 4.3-9.6). The median LOS was longer for those with a MRV (3.0 days [IQR 5.0 days]) compared to those admitted for a non-MRV ED visit (1.5 days [IQR 2.5 days]), $p=0.02$. Conclusion: Pediatric patients who present to the ED with a MRV are more likely to be admitted to hospital and when admitted have a significantly longer LOS.

EP03 Experiential and rational clinical decision making: a survey to determine decision-making styles of paramedics

J.L. Jensen, ACP MAHSR, L.A. Calder, MD, MSc, FRCPC, M. Walker, ACP, A. Travers, MSc, MD, W. Tavares, ACP, PhD(c), A. Bienkowski, BSc, Medical Student, P. Croskerry, PhD, MD; EHS Operations Management

Keywords: clinical decision making, emergency medical services, emergency medicine

Introduction: Two major processes underlie human decision making: experiential (intuition) and rational (conscious and deliberate). The predominant process that paramedics use for making clinical decisions is unknown. Our objective was to determine paramedics' preferences toward and perceived ability to use experiential and rational thinking. Methods: Paramedics employed in a provincial ground ambulance system voluntarily completed a paper survey. The survey included eight demographic questions and the Rational Experiential Inventory40, a 40-question validated psychometric tool, scored on a 5-point Likert scale. Twenty questions were on each thinking style, of which 10 assess favourability and 10 assess ability to use that style. Analysis included descriptive statistics and $t$-tests to determine differences in overall thinking style scores and favourability toward and ability to use each style. Differences in thinking styles were evaluated within demographic variables with $t$-tests and ANOVA. Results: The response rate was $96.1 \%$ (904/941). Most participants were male $(n=628,69.5 \%)$, primary or advanced care paramedics ( $\mathrm{PCP} n=502,55.5 \%$; ACP $n=$ 294, 32.5\%), median age 36 years (IQR 29-42), and median years of experience 10 (IQR 4-16). The mean rational scores were higher than experiential: $3.86 / 5$ (95\% CI 3.83-3.87) versus 3.41/5 (95\% CI $3.38-$ $3.44), p<0.001$. Participants scored their ability to use rational thinking higher than experiential (3.93/5 [95\% CI 3.90-3.96] v. $3.60 / 5[95 \%$ CI 3.58-3.63], $p<0.001)$ and more favoured rational than experiential (3.79/5 [95\% CI 3.76-3.82] v. 3.22/5 [95\% CI 3.18-3.26], $p<0.001)$. Males scored experiential thinking higher than females. The following subgroups preferred rational thinking over their colleagues: younger age, ACPs, less work experience, and those working in urban areas. Conclusion: Paramedics perceive that they have the ability to use and favour rational over experiential thinking. Future research includes determining differences in thinking styles of paramedics with work experience and students. This study adds to what is known on paramedic decision making and is important for developing continuing education and clinical support tools.

EP04 National survey of physicians on the need for and sensitivity of a clinical decision rule to identify elderly patients at high risk of functional decline following a minor injury

K. Abdulaziz, MSc, J.J. Perry, MD, MSc, CCFP(EM), J. Brehaut, $\mathrm{PhD}, \mathrm{M}$. Taljaard, PhD, M. Emond, MD, MSc; Ottawa Hospital Research Institute

Keywords: activities of daily living, emergency medicine, functional decline, geriatrics

Introduction: Elderly patients ( $\geq 65$ years of age) frequently visit the emergency department (ED) for minor injuries. Large proportions of these patients are not assessed for functional status (i.e., ability to walk and care for themselves) and are discharged home. Many such patients experience persistent functional decline even up to 6 months postinjury. Our objectives were to conduct a mail survey of Canadian physi- 
cians to determine (1) the required sensitivity for a clinical decision rule to assess for functional decline at 6 months postinjury and (2) a clinically significant point drop in activities of daily living (ADL) and instrumental activities of daily living (IADL) scores to define functional decline for the purposes of a planned clinical decision rule. Methods: Following qualitative and cognitive interviews, a survey questionnaire was developed. A random sample of 534 emergency physicians, geriatricians, and family physicians registered in the Canadian Medical Directory was then selected (178 from each group). Half of the physicians from each group were randomly selected to receive a monetary token of appreciation with the first survey. We pilot tested the survey on local physicians prior to mass mailout. In a modified Dillman technique, we distributed a prenotification letter, the survey questionnaire, and up to four follow-up contacts, including a final special contact using express courier to nonresponders. Results: A total of $265(57.0 \%)$ physicians completed the survey. The overall median required sensitivity for a clinical decision rule to identify injured seniors at high risk for a functional decline 6 months postinjury was $80 \%$ (interquartile range [IQR] $80-90 \%$ ). The median sensitivity required by family physicians $(80 \%$, IQR $75-88 \%)$ was significantly lower than that required by emergency physicians $(90 \%$; IQR $80-90 \%$, $p=0.0002)$ and geriatricians ( $85 \%$; IQR: $80-90 \%, p=0.0008)$. Considering all $14 \mathrm{ADL} / \mathrm{IADLs}$, physicians reported a median point drop of 5 (IQR 4-7) to imply functional decline. If only ADLs were considered, a median point drop of 2 (IQR 2-4) was considered a clinically important change, indicative of functional decline. Conclusion: Our results indicate that $75 \%$ of physicians would consider a drop in function of no less than 4 points in the ADL/IADLs as clinically significant and that $75 \%$ of physicians would require a tool with sensitivity no greater than $90 \%$ to detect patients at risk for a larger decline.

EP05 CLEARER - Canadian learning in EBM among Royal College emergency residencies: a national survey of program directors J.M. Bednarczyk, MD, M. Pauls, MD, J. Fridfinnson, MD, E. Weldon, MD, FRCPC; University of Manitoba

Keywords: emergency medicine, evidence-based medicine, medical education

Introduction: Recent surveys suggest that few emergency medicine (EM) training programs outside of Canada have formal evidence-based medicine (EBM) or journal club curricula. Our primary objective was to determine the methods of EBM training in Canadian Royal College (RC) EM residencies. Methods: A paper based survey containing 5point Likert scale, binary, and open-ended questions was distributed to the 14 RC-EM program directors in Canada. Survey content was refined by consensus discussion during local focus group meetings and informal collaboration with national EBM experts in EM. Proportions of respondents (\%), median, and IQR are reported. Results: The response rate was $93 \%(13 / 14)$. Most programs (85\%) had established EBM curricula. Curricula content was delivered primarily via journal club, with a median response of 10 or more sessions per year. Less than half of journal clubs were led consistently by EBM experts (46\%). Some programs (31\%) did not use a critical appraisal tool in their sessions. Additional teaching formats included didactic and small-group sessions, self-directed e-learning, EBM workshops, and library tutorials. Fifty-four percent of respondent programs operated program websites linking external EBM educational resources. Podcasts, blogs, and online journal clubs were valued for EBM teaching roles, including creating exposure to literature (4, IQR 1.5), linking literature to clinical practice experience (4, IQR 1.5), and framing outcomes with expert opinion (4, IQR 1.5) (1-no merit, 5-strong merit). The majority of respondents supported the creation of a national unified EBM educational resource (4, IQR 1) (1-no support, 5-strongly support). Conclu- sion: Canadian RC-EM training programs have established EBM teaching curricula and deliver content primarily via journal club. Program directors supported the nationalization of EBM teaching resources. A growing usage of e-resources may represent an avenue to facilitate future collaborative EBM educational efforts in Canada.

EP06 Is achieving Ontario's emergency department length of stay performance targets associated with improved patient outcomes following discharge?

M. Schull, MD, MSc, A. Guttmann, MDCM, MSc, M. Vermeulen, MSc, T. Stukel, PhD; Sunnybrook Research Institute

Keywords: ED performance targets, emergency medicine

Introduction: Many jurisdictions have adopted targets for ED length of stay (LOS). Yet empirical evidence linking better target performance with better patient outcomes is lacking. We tested, among ED patients who ultimately go home from the ED, whether those being seen during a shift when a greater percentage of all patients meet the ED LOS targets have a lower risk of adverse events following discharge. Methods: A retrospective cohort study using health administrative data in Ontario EDs (2007-2010) of visits resulting in discharge home or left without being seen. For each visit, the main exposure was the percentage of all patients meeting their ED LOS target during the same shift in the same ED. Shift-level ED LOS performance was categorized as $<80 \%$; 80 to $<90 \%$; 90 to $<95 \%$; and 95 to $100 \%$ of all patients meeting their target. Main outcomes were death or hospital admission in the 7 days following the visit. Important covariates were controlled for. Results: We included 13 million ED visits at 134 EDs. Seven-day mortality and hospitalization rates were $0.11 \%$ and $2.4 \%$ (high-acuity patients) and $0.021 \%$ and $0.64 \%$ (low-acuity patients), respectively. Presenting to an ED when ED LOS targets were being met was associated with a 10 to $40 \%$ reduction in the risk of adverse outcomes. Adjusted odds ratios $(95 \% \mathrm{CI})$ for patients seen on shifts when $<80 \%$ of ED patients met their LOS targets, among high- and low-acuity patients, respectively, were $1.17(1.06,1.28)$ and 1.44 $(1.22,1.70)$ for death and $1.14(1.12,1.16)$ and $1.36(1.32,1.40)$ for admission, when compared to shifts when $>95 \%$ of patients met their targets. The risk of adverse outcomes decreased with increased attainment of ED LOS targets. Conclusion: Achieving ED wait time targets was associated with a reduced risk of death or hospitalization after ED discharge. This study provides empirical support for ED LOS targets as a means to improve patient outcomes.

EP07 Comparing the characteristics and management of patients with hypertension at a community versus an academic emergency department

A. Zia, MD, A.Y. Wong, MD, C. Atzema, MD, MSc; Institute for Clinical Evaluative Sciences

Keywords: emergency department, emergency medicine, hypertension, hypertensive urgency

Introduction: Hypertension is a common reason to present to the emergency department (ED). We compared the characteristics and management of patients with hypertension at a community and an academic ED. Methods: In this chart review conducted at an academic (Sunnybrook Health Sciences Centre [SHSC]) and a community (Markham Stouffville Hospital [MSH]) ED, patient demographics, symptomatology, and ED and post-ED management were collected on patients seen between April 2010 and March 2011 with a final ED diagnosis of hypertension. Descriptive statistics were used to describe the cohort by site. Results: Among 545 qualifying patients, 264 were seen at SHSC and 281 at MSH. Mean age was 66.2 and 62.4 at SHSC and MSH, respectively, with $61.7 \%$ and $58.0 \%$ females. Mean 
SBP/DBP at triage was similar: 189/101 at SHSC and 183/98 at MSH. There were differences in the proportion of patients who checked their BP (via home or pharmacy measurement) prior to the ED visit $(45.5 \%$ versus $62.3 \%$, respectively) and patients presenting with symptoms ( $72.6 \%$ versus $19.9 \%$, respectively). At SHSC, 39.8\% (95\% CI, 33.8 to 46.0) of patients received ED medication for their hypertension, more than the $30.6 \%(95 \% \mathrm{CI}, 25.3$ to 36.4) at MSH. More patients received a prescription upon ED discharge from SHSC (31.4\%; 95\% CI 25.9 to $37.4)$ than from MSH (19.9\%; 95\% CI 15.4 to 25.1$)$. The difference in presenting and final ED SBP/DBP at the two sites was similar $(27 / 18$ at SHSC versus 32/16 at MSH); however, a higher proportion of patients were admitted at SHSC compared to MSH $(15.5 \%$; $95 \% \mathrm{CI}$ 11.4 to 20.5 versus $5.7 \%$; $95 \%$ CI 3.3 to 9.1 , respectively). Conclusion: While patients seen at the academic and community site had similar blood pressure values, the majority of patients seen at the community ED came for asymptomatic hypertension found on a blood pressure reading. There was substantial variation in ED management and disposition of these patients between the two sites.

EP08 The use of tranexamic acid for bleeding trauma patients at a Canadian academic trauma centre

M. Ott, MD, M.Y. Woo, MD, R. Grainger, BSc, LLB, MBA, I.G. Stiell, MD, MSc, FRCPC; University of Ottawa

Keywords: emergency medicine, injury, resuscitation, trauma Introduction: Tranexamic acid reduces the mortality rates of bleeding trauma patients when given within 3 hours of injury. This study assessed the use of tranexamic acid in trauma resuscitation at a Canadian academic trauma centre. Methods: A formal health records review was performed over an 18-month period from June 15, 2010, to September 30, 2011. Inclusion criteria included any adult trauma patients who either had or were at risk of significant bleeding based on a standard set of clinical parameters. A single reviewer identified cases and abstracted the data into a standardized electronic database. Ten percent of charts were independently reviewed for validity. Descriptive and Fisher exact test statistics were used. Results: Seventy trauma patients met the inclusion/exclusion criteria with a mean age of 40 years (range 18-89); 77.1\% were male, and 99\% presented within 3 hours of injury. A trauma code was initiated for $86 \%$ of patients and $8.6 \%$ died, none of whom received tranexamic acid. Tranexamic acid was given to only $9(13 \%)$ patients, each with his or her own dosing regimen. Only $2(2.8 \%)$ received tranexamic acid within 3 hours from their injury, while the rest received a first dose well after 4 hours from injury. Administration of tranexamic acid correlated with patients who sustained blunt or penetrating trauma with a Glasgow Coma Scale under $13(p=0.05)$, who had a rapid sequence intubation in the emergency department $(p=0.05)$, an amputation or severe extremity trauma $(p=0.07)$, or those who received blood products $(p=0.08)$. Conclusion: The majority of eligible bleeding trauma patients are not given tranexamic acid. Moreover, those patients who are given tranexamic acid are receiving doses greater than 3 hours from injury, thus out of the time window for benefit. Trauma resuscitation protocols incorporating tranexamic acid may improve its use.

EP09 An exploration of Canadian emergency physicians' and residents' knowledge surrounding CT radiation dosing and risk D. Barbic, MD, S. Barbic, MSc, J. Dankoff, MD; McGill University

Keywords: computed tomography, emergency medicine, radiation Introduction: To describe the current state of knowledge of Canadian emergency physicians (EMPs) and trainees (EMRs) regarding CT radiation dosing and its associated risks. Methods: Focus groups of EMPs and EMRs $(n=21)$ at the McGill University Health Centre (Montreal, QC) were convened. Subsequently, an online survey was distributed to
Canadian EMPs and EMRs $(n=638)$. Descriptive statistics were used to analyze the results. Results: Four themes emerged from the focus groups: (1) physician knowledge about the quantitative risks associated with CT dosing is limited; (2) risk management strategies used by EMPs/EMRs vary depending on specific patient populations (children, women of reproductive age, or pregnant women); (3) direct communication with patients about risks associated with $\mathrm{CT}$ is rare; and (4) EMPs/EMRs have a desire to learn more about $\mathrm{CT}$ dosing and risks. The overall survey response rate was $49.8 \%(638 / 1,281)$. The mean age of respondents was 40.9 years (SD 9.9), and $68.5 \%$ were male. Of all respondents, $79.9 \%$ were EMPs, $53.4 \%$ of all respondents practiced in urban academic EDs, and the average time practicing was 10.7 years (SD 9.6); 79.6\% of respondents correctly identified the lifetime attributable risk (LAR) of malignancy due to CT in adult patients, but only $49.6 \%$ were able to identify the LAR for children. Appropriate risk management strategies were reported by $83 \%$ of respondents for at-risk populations. Only $36 \%$ of respondents reported communicating the risks of CT to a majority of their patients. Respondents identified smartphone applications (46.3\%) and Web-based modules $(26.0 \%)$ as their preferred methods of knowledge translation on this topic. Conclusion: Canadian EMPs and EMRs demonstrated important, identifiable gaps in knowledge surrounding CT radiation dose and risk. This study identified several key areas for future physician education and the preferred modalities for these knowledge translation efforts.

EP10 Health literacy and numeracy in the emergency department K. Milne, MD, N. Palalia, BSc, K. Nicholson, MSc, C.A. Camargo, MD, C. Carpenter, MD, A. Dixon, MD, D. Ng, MD; South Huron Hospital

Keywords: communication, literacy, numeracy

Introduction: Although health literacy is an important determinant of health, there has been limited research on the topic in Canada, particularly in the busy emergency department (ED) setting. Most tools to assess health literacy are not practical for use in the ED. The objective of this study was to quantify health literacy and numeracy in the ED setting using a rapid, validated instrument. Methods: A total of 301 total patients were surveyed from three EDs during the summer of 2012. Consecutive patients who met inclusion criteria were approached by a medical student. A seven-word REALM-SF was administered with a numeracy component. Data were analyzed with Stata 10.0. Results: The average age of the respondents was 54 years, ranging from 18 to 92 years. Approximately half $(53 \%)$ of the respondents were male, with $43 \%$ having a degree or diploma. All seven literacy words were correctly answered by $59 \%$ of the respondents. The number of correct answers stratified patients into one of four grade levels: grade 3 or less $(1 \%)$, grade $3-6(7 \%)$, grade $7-8(33 \%)$, and grade 9 or higher $(59 \%)$. The percentage of patients getting the four numeracy questions correct was zero $(27 \%)$, one $(22 \%)$, two $(27 \%)$, three $(15 \%)$, and four $(10 \%)$. It took an average of 11.4 seconds to complete the REALM-SF and 68 seconds to answer the numeracy questions. Conclusion: This study quantifies the health literacy and numeracy of patients presenting to the ED. It also demonstrates a brief tool that can be used in the ED. Health care professionals should consider patients' health literacy and numeracy when communicating with their patients in the ED.

EP11 Patterns of injury in recreational curlers

D.K. Ting, BSc, R.J. Brison, MD, FRCPC; Queen's University School of Medicine

Keywords: emergency medicine, injury prevention, recreational curling 
Introduction: Curling is a sport played throughout Canada that is generally considered safe. Nevertheless, there are no published reports on the occurrence of acute injuries in recreational curlers. Our objective was to describe the epidemiology of acute injuries in curling in the hope of informing the need for, and perhaps design of, injury prevention strategies. Methods: Injured curlers from 1993 through 2010 were identified by searching an ongoing emergency department (ED) injury surveillance program that identifies all persons presenting to either of Kingston's two EDs with an acute injury. This program collects information on the circumstances of the injury event and on the resulting anatomic injury. A secondary mail survey, to collect additional information on the injury event and also opinions on a series of preventive strategies, was sent to curlers treated in the most recent 10 years. Regional curling clubs were contacted for membership information to permit estimates of the number of curlers and their age/gender distribution. Injury severity was classified as major (hospital admission and/or concussion, intracranial injury or fracture requiring reduction), moderate (dislocations, musculoligamentous tears, fractures not reduced), or minor. Results are described using estimates of rates and proportions. Results: A total of 223 injured curlers were identified in the full 17year survey period and 104 for the 10-year secondary survey period, of whom $60(58 \%)$ returned the survey. We estimate that there are 1,100 recreational curlers annually in the Kingston region, giving a crude injury rate estimate of $11.9 / 1,000$ curlers/yr (95\% CI; 10.4, 13.6). Falls on the ice surface accounted for $87 \%$ of injuries, with injuries to the head $(30 \%)$, forearm/wrist (23\%), shoulder/humerus (17\%), and back (11\%) being most frequent. Nearly $40 \%$ of injuries were classified as major $(19 \%)$ or moderate $(20 \%)$ severity. In the secondary survey, $38 \%$ of responders reported a problem related to footwear (grippers or sliders) as contributing to their fall. Thirty-four percent had curled $<4$ years. Use of proper footwear was recommended as mandatory by $74.4 \%$. There was little support for use of helmets or of wrist protection. Conclusion: Curling injuries are not common ED presentations, although they can be severe. The lead recommendation for prevention of falls when curling is the use of sport-specific footwear.

EP12 The effect of implementing high-sensitivity troponin testing on operational efficiency in three large urban emergency departments

K. Crowder, MD, T. Jones, BSc, D. Wang, PhD, S. Clark, MD, G. Innes, MD, E. Lang, MD, J. McMeekin, MD, K. Lonergan, BSc, M. Graham, MD, MSc, A. McRae, MD; Alberta Health Services

Keywords: ED flow, emergency medicine, high-sensitivity troponin, resource utilization

Introduction: High-sensitivity troponin $\mathrm{T}$ (hsTnT) assays detect myocardial injury sooner, raising the possibility of improved throughput times for emergency department (ED) assessment of patients with suspected acute myocardial infarction (AMI). Few studies have explored the system-wide impact of the more sensitive, less specific hsTnT assay on resource utilization. In this study, we evaluate the influence of hsTnT implementation on ED length of stay (LOS), consultation, and admission rates for patients with suspected AMI. Methods: This control-pre-post design study involved patients presenting to three Calgary EDs evaluated using hsTnT or its predecessor, conventional TnT. The cutoff value used for diagnosis of AMI was $50 \mathrm{ng} / \mathrm{mL}$. Data were collected from the ED information system database for the periods February 12, 2011, to April 22, 2011 (Ctrl); November 20, 2011, to January 28, 2012 (Pre); and February 12, 2012, to April 21, 2012 (Post). Subjects included ED patients, excluding physicianconfirmed STEMIs, who had at least one troponin drawn as a marker for suspected AMI. Nonparametric and parametric bivariable statistical tests were used as appropriate. Results: We analyzed data from 6,650 (Ctrl), 6,866 (Pre), and 5,754 (Post) patients evaluated with TnT assays. These represent $15.5 \%, 15.2 \%$, and $12.4 \%$ of all ED visits in the Ctrl, Pre, and Post periods, respectively. Demographic characteristics were similar between the groups. Median ED LOS for patients evaluated with a TnT assay decreased following hsTnT implementation (6.60 hours in Ctrl and Pre v. 6.10 hours Post, $p<0.001$ ). The proportion of patients with three troponin assays ordered decreased from $3.4 \% \mathrm{Ctrl}$ and $3.1 \%$ Pre to $2.0 \%$ Post $(p<0.001)$. There was no statistically significant change in cardiology consultations or admissions following hsTnT implementation. Conclusion: Implementing hsTnT testing with an equivalent cutoff value to the prior-generation TnT assay decreased ED LOS for patients with suspected AMI and did not increase cardiology resource utilization. This may be attributable to a carefully considered implementation strategy.

\section{EP13 EMT-basic acquisition and transmission of 12-lead ECG using a novel device (ReadyLink ${ }^{\mathrm{TM}}$ )}

J.M. Goodloe, MD, A.O. Arthur, PharmD, T. Rhodes, BS (in progress), P. Holder, MD, J.O. Winham, RN, BSN, S.H. Thomas, MD, MPH; The University of Oklahoma School of Community Medicine

Keywords: emergency medicine, EMS, 12-lead ECG, telemetry Introduction: Early detection of ST segment elevation myocardial infarction (STEMI) enabled by EMS-performed 12-lead ECGs can improve the timeliness of definitive interventional care. Traditionally, 12-lead ECG acquisition and analysis has been performed by advanced life support (ALS) paramedics. Many EMS systems lack ALS capabilities, operating solely with emergency medical technician (EMT)Basics. This study's purpose is to evaluate if EMT-Basics without prior 12-lead ECG acquisition and telemetry transmission training can attain and maintain those skills using a novel 12-lead ECG device (ReadyLink ${ }^{\mathrm{TM}}$ ). Methods: Fifty-eight EMT-Basics without prior 12lead ECG acquisition and telemetry transmission experience were enrolled. Education regarding 12-lead ECG acquisition and telemetry transmission utilizing the study device (ReadyLink ${ }^{\mathrm{TM}}$ ) was delivered in a classroom setting and consisted of a 25 -minute training video. Study subjects were then allowed hands-on practice with the device for up to 15 minutes. Using volunteer human subjects to simulate patient encounters, study subjects acquired and telemetry transmitted 12-lead ECGs to a study website using a proprietary transmission system (LifeNet ${ }^{\mathrm{TM}}$ ). Twelve-lead ECG acquisition and telemetry transmission evaluations were conducted on the initial day of training, approximately 90 days post initial training, and approximately 180 days post initial training. Study subjects did not utilize 12-lead ECGs in actual patient encounters during the study period. All 12-lead ECGs acquired and telemetry transmitted to the study repository were reviewed by an emergency physician to determine if the quality of the 12-lead ECG was clinically usable for determining activation of emergency interventional cardiology services. Results: During the 6-month study period, the EMT-Basics involved acquired and telemetry transmitted 13212 lead ECGs. A total of 130 (98.5\%) 12-lead ECGs were determined through emergency physician overread to be clinically usable for determining activation of emergency interventional cardiology services. Extreme motion artifact prevented clinically usable data in the remaining $2(1.5 \%)$ 12-lead ECGs. Conclusion: EMT-Basics, inexperienced in 12-lead ECG acquisition and telemetry transmission, are able to successfully achieve clinically usable 12-lead ECG acquisition and telemetry transmission using the ReadyLink ${ }^{\mathrm{TM}}$ device.

\section{EP14 Ontario's Emergency Department Process Improvement Program (ED-PIP): a qualitative evaluation}

M. Schull, MD, MSc, R. Bell, MD, MSc, E. Salkeld, MSc, L. Rotteau, MSc, F. Webster, PhD, C. Hellings, MSc, M. Vermeulen, MSc, M. Zwarenstein, MBBCh, MSc, A. Nigam, PhD, A. Sales, RN, PhD, A. 
Guttmann, MDCM, MSc, B.H. Rowe, MD, MSc, CCFP(EM), B Golden, $\mathrm{PhD}$; Institute for Clinical Evaluative Sciences

Keywords: emergency medicine, process improvement, qualitative Introduction: In 2008, Ontario launched the ER/ALC Wait Time Strategy to reduce emergency department length of stay (ED LOS) and reduce overcrowding. One intervention was the ED Process Improvement Program (ED-PIP), a structured 7- to 8-month program based on Lean management principles to improve patient flow in hospitals. EDPIP was fully implemented in 54 hospitals in four "waves" from 2009 to 2011 , with 5 to 18 hospitals per wave. We sought to identify hospital organizational, cultural, and contextual determinants of perceived success of ED-PIP and to compare these between hospitals that did and did not improve their ED LOS. Methods: We conducted a qualitative evaluation based on semistructured interviews with implementation team members at 10 purposively selected hospitals based on their improvement in median ED LOS after ED-PIP. We selected from among sites that improved the most but also included some sites that did not improve following ED-PIP. We used a thematic framework approach for interviews, and a standard coding framework was developed. Results: Twenty-four interviews were coded among nine physicians, seven nurses, and eight administrators and managers. Analysis of transcripts revealed eight themes, and within each, we identified important subthemes. The themes were the local context and organization's history with quality improvement initiatives, senior leader engagement and support, the role of on-site coaches, preparation and team selection, managing expectations, staff engagement, collaboration and communication, performance measurement, and accountability The most striking differentiating factor between hospitals that saw reductions in ED LOS versus those that did not was the extent of executive (specifically CEO) support. Conclusion: Several key factors were identified as important to the success of ED-PIP. Explicit incorporation of them into the development and implementation of future similar interventions in health care settings could be useful. Qualitative research findings are not intended to be generalizable to an entire population, but, rather, key insights and themes are transferable across similar groups/settings.

\section{EP15 Triage nurse diagnostic accuracy for acute coronary syn- dromes (ACS)}

G. Innes, MD, S. Stackhouse, BSN, E. Grafstein, MD, K.C. Innes, BSc; University of Calgary

Keywords: acute coronary syndrome, chest pain, emergency Introduction: Vancouver Coastal Health (VCH) emergency departments have common triage training and coding systems. VCH triage nurses are taught to place suspected ACS patients into category CV022 (possible ACS), but they could appropriately use one of four other chest pain categories, including CV029 (possible ACS; pain resolved), CV027 (CP + respiratory distress), CV028 (CP + hemodynamic compromise), and CV030 (CP-ripping or tearing). Our objective was to assess triage nurse accuracy in categorizing ACS patients. Methods: This administrative database study included all Vancouver ED patients with a chief complaint of chest pain during a 6-month period in 2012. Discharge diagnoses were coded by emergency physicians or health records technicians based on the index hospital visit. For the primary analysis, triage nurses were considered correct if ACS patients were triaged into category CV022. In the sensitivity analysis, nurses were considered correct if ACS patients were triaged into any of the high-acuity cardiovascular categories above. Sensitivity, specificity, accuracy, and predictive values were determined using standard methods. Results: Of 13,721 patients presenting to six $\mathrm{VCH}$ EDs with chest pain, 5,588 were triaged into category CV022 (ACS), 1,371 into another appropriate cardiovascular category, and 6,762 into lower-acuity or noncardiac categories. Discharge diagnoses included chest pain NYD (8990), ACS (987), dysrhythmia (237), other cardiovascular condition (378), respiratory (820), neurologic (145), mental health (381), musculoskeletal (682), GI (637), or systemic illness (464). ACS sensitivity, specificity, accuracy, PPV, and NPV for category CV022 were $74.4 \%, 61.9 \%, 62.8 \%, 13.1 \%$, and $96.9 \%$. Corresponding diagnostic parameters within the broader set of acute cardiovascular triage categories were $88.6 \%, 52.2 \%, 54.8 \%, 12.6 \%$, and $98.3 \%$. Conclusion: In this population-based sample of patients with chest pain, triage nurses had high sensitivity, placing $89 \%$ of ACS patients in ACS or high-acuity cardiovascular categories; however, $>85 \%$ of patients coded to an ACS category did not have ACS.

EP16 High-sensitivity troponin implementation for patients with suspected ACS increases the rule-out rate while reducing revisits resulting in admission

A. McRae, MD, K. Crowder, MD, T. Jones, MSc, G. Innes, MD, S. Clark, MD, J. McMeekin, MD, D. Wang, PhD, K. Lonergan, BSc, M. Graham, MD, MSc, E. Lang, MD; Alberta Health Services

Keywords: acute coronary syndrome, chest pain, emergency medicine, troponin

Introduction: Highly sensitive cardiac troponin $\mathrm{T}$ (hsTnT) assays are becoming widely adopted, raising the possibility of improved detection of acute myocardial infarction (AMI) combined with enhanced ED throughput for patients undergoing evaluation for possible AMI. This study evaluated the safety, in terms of and 7- and 30-day revisit rates, of implementation of a hsTnT assay using a cutoff higher than the 99th percentile of normal. Methods: This control-pre-post analysis involved a cohort of all patients presenting to three tertiary care adult EDs evaluated using hsTnT or its predecessor, cTnT. The hsTnT assay was implemented on January 31, 2012. Three 10-week periods were utilized: February 12, 2011, to April 22, 2011: control period 1 year prior to hsTnT implementation (CtrllyrPre); November 20, 2011, to January 28, 2012: immediately pre-hsTnT implementation (Immed-pre); and February 12, 2012, to April 21, 2012: immediately post-hsTnT implementation (Post). The hsTnT cutoff value used for diagnosis of AMI was $50 \mathrm{ng} / \mathrm{L}$. Subjects included all ED patients, excluding physicianconfirmed STEMIs, who had at least one cTnT or hsTnT drawn as a marker for symptoms suspicious of AMI. The primary outcomes included 7- and 30-day ED revisits and 7- and 30-day revisits resulting in hospital admission. The secondary outcome was the proportion of suspected ACS patients discharged from the ED following their initial workup. Proportions were compared using Pearson chi-square test. Results: Troponin assays were used to evaluate 6,650 Ctrl, 6,866 Pre, and 5,754 Post patients; demographic characteristics between the groups were similar. A total of 3,736 (56.2\%) Ctrl, 3,830 (55.8\%) Pre, and $3,391(58.9 \%)$ Post patients were discharged from the ED following their initial workup $(p<0.001)$. There was no statistically significant difference in 7- or 30-day return ED visit rates for discharged patients evaluated using the different TnT assays. However, fewer 30 day ED revisits requiring admission were observed following hsTnT implementation (ARR $1.3 \%$, RRR 24\%, $p=0.011$ ). Conclusion: Replacing conventional cTnT with hsTnT is associated with a modest increase in the proportion of suspected ACS patients being discharged from the ED as well as improved decision making reflected by a reduced rate of revisits resulting in hospitalization.

EP17 Closing the circle of care: implementation of a Web-based communication tool to improve emergency department discharge communication with family physicians

C. Hunchak, MD, CCFP(EM), MPH, D. Tannenbaum, MD, CCFP, FCPC, M. Roberts, MD, CCFP, FCFP, H.J. Ovens, MD, B. Borgundvaag, $\mathrm{PhD}, \mathrm{MD}$; University of Toronto 
Keywords: emergency care information systems, emergency medicine, medical informatics application

Introduction: Communication of emergency department (ED) health record information to family physicians (FP) is often suboptimal and can negatively impact on patient care. To improve continuity of care, reduce duplication of investigations, and improve ED-FP relations, we designed and piloted a Web-based notification system to alert FPs of patient ED visits by email and to provide secure online access to visitspecific laboratory and diagnostic information. Methods: This mixedmethods pilot study was completed by nine high-referring FPs to the Mount Sinai Hospital ED in Toronto, Ontario. A pre-pilot chart audit was conducted to determine the baseline rate of paper-based information transfer between the ED and FP offices. A Web-based communication portal was designed by the hospital IT department and piloted by the nine FPs during a 1-year consecutive period. Participants then attended a focus group and completed a written survey regarding its utility and ease of use. Results: A total of 270 patient charts were audited and revealed a low (12\%) baseline rate of complete paperbased information transfer and a $41 \%$ rate of incomplete information transfer between the ED and FP offices following patient ED discharge. The nine FPs accrued 880 patient visits during the 1-year pilot period and accessed patient records online $60.7 \%$ of the time; $14.3 \%$ of unique patient records were visited more than once. The post-pilot survey found that $100 \%$ of FPs were "often" or "always" aware of patient ED visits, used the portal "always" or "regularly" to access patients' health records online, and felt that the Web portal contributed to improved actual and perceived continuity of patient care. Conclusion: Introduction of a Web-based ED visit notification and communication tool significantly improved ED-FP communication and has the potential to improve continuity of care, reduce unnecessary duplication of investigations and referrals, and improve relations between ED physicians and community-based FPs.

EP18 Propofol is officially the "sedative of choice" in Halifax! An update from the Halifax ED procedural sedation registry

S.G. Campbell, MB BCh, P. Froese, CCP, M. Butler, BSc, G.R. Etsell, ACP, L. Alan, CCP, R. MacKinley, ACP, M. Watson, MD, CCFP(EM), K.D. Magee, MD, FRCP, P. Zed, PharmD, G.J. Kovacs, MD, FRCP; Charles V. Keating Emergency and Trauma Centre

Keywords: emergency medicine, paramedic, procedural sedation, propofol

Introduction: The introduction of propofol into the ED for procedural sedation (EDPS) is still opposed in some institutions due to concerns about the potential for dangerous side effects, often by people with little knowledge or experience of emergency medicine. The Halifax EDPS registry has gathered clinical and procedural data on all adult patients undergoing EDPS since 2004. Our report of EDPS between August 1, 2004, and February 28, 2006, showed propofol used in $61.2 \%$, midazolam in $42.5 \%$, and ketamine in $2.7 \%$, with the proportion of propofol increasing as the study period progressed. In our ED, advanced care paramedic EDPS practitioners are free to choose which sedative to use. We suggest that trends in the use of a particular medication by this expert group would identify which sedatives are considered easier to use and less likely to cause complications. Methods: We compared proportions of propofol use to those of other sedatives in the time following our earlier report to see whether the trend toward increasing proportion of propofol use continued and whether any trend was associated with any significant adverse events. Results: Of 3,544 EDPS conducted between March 1, 2006, and November 20, 2012, $3,310(93.4 \%)$ were conducted using propofol as the sedating agent; 183 (5.2\%) were conducted with midazolam and 67 (1.9\%) with ketamine. Hypotension occurred in $97(2.7 \%)$ patients and hypoxia in 92
(2.6\%). No other adverse events were recorded, and all patients recovered fully by the end of the procedure. Conclusion: Propofol has clearly become the sedative of choice for ACP-administered EDPS in our institution. This does not appear to be at the expense of significant adverse events.

\section{EP19 Is propofol for emergency department procedural sedation} any less safe in older teenagers than in older patients?

S.G. Campbell, MB BCh, M.B. Butler, BSc, S. MacPhee, MD, FRCP, P. Zed, PharmD, P. Froese, CCP, M. Watson, MD CCFP(EM); Charles V. Keating Emergency and Trauma Centre

Keywords: emergency medicine, procedural sedation, propofol Introduction: Propofol is an accepted standard for emergency department procedural sedation (EDPS) in adults. Its use in children remains controversial. We believe that if the risk of propofol is higher in children, we would expect adverse events to increase in patients below the age of 19. Methods: This is a retrospective study of records from the Halifax Procedural Sedation Registry, collected between January 22, 2006, and October 29, 2012. Patients undergoing EDPS using propofol were divided into those 16 to 19 (teenagers) and those older than 19 (adults). Our primary objective was to investigate whether adverse events occurred more frequently in younger patients receiving propofol. Results: Of 3,313 propofol sedations, 185 were on teenagers and 3,125 on adults (mean age 51.4 years). Teenagers were significantly healthier (OR of being ASA 1: 17.4, 95\% CI, 7.28, 54.43) and were less likely to undergo cardioversion (OR: $0.12,95 \%$ CI $0.14,0.46$ ). Most procedures were for orthopedic indications (74.1\%) (OR of orthopedic indications in teenagers: $1.45,95 \%$ CI 0.99, 2.17). Duration of EDPS and patient satisfaction were similar. Adverse events were 2.23 times more likely in the adult group (95\% CI: $1.35,3.88)$, hypotension $(6.5 \%$ in teenagers v. $15.2 \%$ adults: OR $0.39,95 \% \mathrm{CI}$ : $0.2,0.7)$. There was no significant difference in the incidence of hypoxia or airway interventions. Teenagers received more propofol on average than the adults $(0.214 \mathrm{mg} / \mathrm{kg} \times$ min versus $0.174 \mathrm{mg} / \mathrm{kg} \times \mathrm{min}$; $p=0.041)$. Conclusion: Teenage patients receiving EDPS with propofol were healthier than older patients, had a lower incidence of adverse events, and tolerated larger doses of medication than older patients. Satisfaction and duration of EDPS were similar. Concerns about propofol use in younger patients have not been supported by this study. We believe these findings justify a trial of use in younger teenagers needing EDPS.

EP20 Do certain coping styles influence burnout and emergency health care staff wanting to leave their career prematurely? A mediation analysis

M. Howlett, MD, K. Doody, MA, J. Murray, MD, D. Leblanc-Duchin, BSc, MA, PhD, J. Fraser, Bachelor of Nursing, P.R. Atkinson, MD FCEM; Discipline of Emergency Medicine, Memorial University

Keywords: burnout, emergency department administration, emergency medicine

Introduction: Associations between coping styles in stressful situations and levels of burnout have been documented in health care professionals. Understanding how coping styles and burnout can work together to impact professional outcomes has not been studied. The authors investigated the mediated effects of coping styles through levels of burnout, on wanting to leave emergency health care (ED) professions. Methods: A total of 286 ED professionals completed the Maslach Burnout Inventory (MBI), the Coping Inventory for Stressful Situations (CISS), and a demographic questionnaire. Mediation analysis studies the indirect effect of a factor on an outcome, mediated through another factor. A multiple mediator model was hypothesized 
where the MBI subscales work in conjunction to mediate the effects of the CISS variables on wanting to leave an ED profession. A total of three mediation models were examined as outlined in Baron and Kenny (1986). Results: Two of the three models showed evidence of a significant mediated effect. Higher emotion-oriented coping (CISS) was associated with higher emotional exhaustion (MBI), which in turn was associated with a higher likelihood of wanting to leave (indirect $=$ $0.059,95 \%$ bootstrap CI $=0.029,0.094)$. Conversely, increases in task-oriented coping (CISS) was associated with decreased emotional exhaustion (MBI), which was associated with a lower likelihood of wanting to leave (indirect $=-0.042,95 \%$ bootstrap CI $=-0.08,-0.02$ ). Conclusion: The results demonstrate a directional relationship between coping styles and wanting to leave an ED profession, a consequence that may affect staff well-being and human resources. An intervention targeting the coping styles of ED professionals may reduce levels of burnout, which in turn may reduce the number of staff wanting to leave.

EP21 Block first, opiates later? The use of the fascia ilica block for patients with hip fractures in the emergency department: a systematic review

A. Chesters, MD, P.R. Atkinson, MD FCEM; Regional Trauma Centre, Cambridge University Hospital

Keywords: emergency medicine, regional anesthesia, trauma Introduction: Patients who suffer hip fractures often require large doses of opiate analgesia during their initial assessment. We performed a systematic review of the literature to determine the efficacy of the fascia iliaca block in providing adequate analgesia to patients presenting with hip fracture in the emergency department. Methods: EMBASE, PubMed, CINAHL, and Google Scholar were searched. Free text keywords for population, intervention, and outcome were identified to create a search string. The reference lists from articles identified in the primary electronic search were hand-searched. The search strategy was repeated using the System for Information on Grey Literature in Europe. Potentially eligible studies were identified based on review of the title and abstract. If eligibility was unclear from the title and abstract, the full text was examined. Randomized controlled trials comparing the fascia iliaca block with standard analgesia were included. A standardized appraisal of the methodological quality of the studies was performed. Results: Thirty-nine articles were identified, of which 13 were duplicates. Of the remaining 26,14 were relevant to the question and suitable for further sorting. Of these 14 papers, 2 were randomized controlled trials, 5 were cohort studies, and 4 were reports of audit of practice. There were 3 abstracts of conference submissions, which were descriptions of reviews or service development projects. The 2 randomized controlled trials showed statistically significant superior or equal pain relief between the fascia iliaca block and other forms of acute pain relief. Conclusion: The fascia iliaca block could have an important role in first-line pain control for patients presenting to the emergency department with a hip fracture. There is potential to reform the acute management of this common group of patients, which could lead to decreased opiate use in this elderly population.

\section{EP22 Use of an electronic case log to assess resident training in a Canadian emergency department \\ D.S. King, MD, E. Grafstein, MD; St. Paul's Hospital}

Keywords: educational measurements/methods, emergency medicine, internship and residency

Introduction: Little is known regarding the volume and variety of patient presentations and procedures seen by Canadian emergency medicine residents as they progress through training. Compliance with tracking systems requiring manual data input has not been shown to be effective. Methods: We reviewed ED electronic health record (EHR) data at a tertiary care teaching hospital of Canadian College of Family Physicians - Emergency Medicine R3 residents over 2 training cohort years (2009-2011). Residents assign themselves to patients, thereby linking them to patient triage scores, presenting complaints, discharge diagnoses, and all performed procedures that are entered for patients. Data completeness was validated by surveying on-shift residents regarding EHR use. Results: Residents logged an average of 235 patient encounters (range 97 to 376) during an average of 45 training shifts (range 30 to 59) at their home hospital. Survey results indicated that residents log most encounters (mean 90\%). Residents logged increasing numbers of patients per hour as they progressed through training $(0.54$ v. 0.67 v. 0.72 in sequential thirds of training; $p<0.01)$. A similar trend was observed in the total numbers of high-acuity patients (CTAS 1-2) logged by residents (5.93 v. 8.29 v. 10.14 in sequential thirds of training; $p<0.01$ ). Residents logged significantly fewer procedures than the average emergency physician (EP) (30 v. 83 during 45 shifts, $p<0.001$ ). There was no statistically significant difference in case mix seen by residents and EPs. Gastrointestinal, cardiac, and orthopedic complaints were the most commonly seen cases. Conclusion: An existing EHR can be used to provide important resident information regarding (1) volume of patients, (2) trends over time, and (3) number and type of procedures. These data can provide feedback to learners and can help program leaders to tailor individual educational experiences.

EP23 Trends in emergency department use in Ontario, 2003 to 2009: correlations between determinants of health and Canadian triage acuity scores

J.M. Caudle, MD, N. VanStone, PhD, A. Wynn, BSc, S. Biro, MPH, T. Williamson, PhD, P. Belanger, PhD, K. Moore, MD, MPH; Department of Emergency Medicine, Queen's University

Keywords: determinants of health, emergency medicine, triage acuity scores

Introduction: Emergency department (ED) crowding continues to pose challenges for health care in Ontario. We explore trends in the acuity of patients presenting at EDs in relation to their relative socioeconomic status (SES) to increase our understanding of populations accessing ED services, thus enabling evidence-based placement of health care resources in areas of need. Methods: We applied a rigorous proxy of SES using a census-based deprivation index (DI) to Ontario ED admittance data from 2003 to 2009. Data were extracted from the National Ambulatory Care Reporting System (NACRS) data set. ED visits were ranked into quintiles by relative deprivation, and a deprivation map by dissemination area was generated for Ontario. Correlations between deprivation and acuity at triage are investigated. Results: The rate of ED use remained relatively stable at approximately 415 visits per 1,000 population for 2003 to 2009, and ED visits of low relative to high acuity decreased from $55.6 \%$ to $44.6 \%$. There was little variation in patterns of relative patient deprivation: the most deprived quintile generated nearly twice as many visits as the least deprived for all years. For specific triage acuity scores, the relative number for most versus least deprived were always greater than 1.7 and as high as 3.4 for nonurgent conditions. Conclusion: Strategies to reduce crowding by reducing patient input need to focus on our most vulnerable populations; placing appropriate primary health care and health resources may reduce, in the short term, those seeking health services for conditions of low acuity at the ED. 


\section{Posters}

P01 Outcome feedback within emergency medicine training programs: an application of the theory of deliberate practice

T. Dalseg, MD, L.A. Calder, MD, MSc, FRCPC, A. Lee, PhD, J. Walker, BSc, J.R. Frank, MD, MA(Ed), FRCPC; University of Ottawa, Department of Emergency Medicine

Keywords: education, emergency medicine, outcome feedback Introduction: Outcome feedback is the process of finding out what happened to a patient after evaluation and treatment within the emergency department. Research on the topic is minimal and has never been performed within Canadian emergency medicine training programs. The objective was to perform the first national survey of Canadian FRCP (Fellow of the Royal College of Physicians) EM (emergency medicine) residents, pediatric EM residents, and their respective program directors to determine if outcome feedback is obtained for patients treated in the emergency department. The study simultaneously investigated and compared the perceived educational value of outcome feedback by residents and program directors. Methods: We distributed surveys to all Canadian FRCP and pediatric EM programs. Using a modified Dillman method, program directors received an online survey while residents received a paper version distributed by their chief resident during a mandatory academic session. The piloted surveys contained 20 and 19 questions, respectively, regarding outcome feedback systems, educational values, and demographics. $P$ values were calculated using Student $t$-test and Fisher exact test. Results: Completed surveys were collected from 188 of 237 eligible residents $(79.3 \%)$ and 21 of 24 program directors $(87.5 \%)$ for an overall response rate of $80.0 \%$. Mandatory patient follow-up was not present in any EM training program for admitted or discharged patients. Followup was performed electively by $91.4 \%$ of residents for patients admitted to the hospital and by $49.1 \%$ of residents for patients discharged home. A majority of residents $(77.5 \%)$ believed that some type of patient follow-up should be mandatory compared to $42.8 \%$ of program directors $(p=0.005)$. The perceived educational value of outcome feedback was 5.8/7 for residents and 5.1/7 for program directors (difference $0.70 ; p=0.003)$ based on a 7-point Likert scale $(1=$ not important; 7 = very important). Conclusion: While Canadian EM training programs do not mandate follow-up, it is performed electively by the majority of residents surveyed. Residents place a significantly greater educational value on outcome feedback than their program directors and believe that follow-up should be a mandatory component of Canadian EM residencies.

P02 Hypertension: the understood serial killer in the backyard; the underdiagnosed, undertreated, and unvanquished disease B.E. Levy, MD, I.J. Preyra, MBA, MD, M. Mercuri, PhD(c); McMaster University

Keywords: emergency department, emergency medicine, hypertension, prevention

Introduction: Hypertension has been identified as a major risk factor in lethal and disabling vascular diseases. Despite increasing dependence on emergency providers for much routine health care, little is known about the implications of incidentally encountered elevated blood pressure in the emergency department. We sought to better characterize the relationship between ED triage blood pressure (TBP) and a patient's ambulatory baseline blood pressure. We also examined the relationship between pain and blood pressure and other comorbidities. Methods: We performed an observational study of 425 patients in a large Canadian emergency department. We surveyed subjects' current health status prior to taking triage vitals.
Those found with TBP $>150 / 90$ were followed for repeat measurements during their visit using a serial blood pressure measurement device shown to closely predict ambulatory blood pressure. Our findings characterize the signficance of TBP relative to usual blood pressure and the adequacy and effectiveness of current primary care and emergency department referral. Results: The prevalence of previously diagnosed hypertension was $34.8 \%$. Additionally, we found an incremental prevalence of $16.9 \%$ undiagnosed hypertension, even after serial measurement $\approx 85$ minutes later. Over one-third of newly diagnosed patients suffered from stage 2 hypertension. Only $42.5 \%$ of previously diagnosed hypertensive patients were controlled to guidelines. Only a small subsegment of subjects (16\%) demonstrated precipitous pressure drops with repeated measurement $(-21 \mathrm{~mm} \mathrm{Hg} /-$ $10 \mathrm{~mm} \mathrm{Hg}$ ), perhaps illustrating "white coat hypertension." However, the greater majority of patients with elevated TBP evidence only slight moderation $(-6.6 \mathrm{~mm} \mathrm{Hg} /-1.2 \mathrm{~mm} \mathrm{Hg}$ ) on serial measurement. Overall, of patients presenting with TBP $>150 / 90$, over $76 \%$ continued hypertensive after serial measurements. We found no association between pain and hypertension. Conclusion: Absent heightened commitment among primary care physicians to achieve guidelines and alternative referral strategies among emergency practitioners, unchecked hypertension will drive an unsustainable drain on health care resources and result in needless patient suffering.

P03 Gender-specific differences in the prevalence and emergency department management of anaphylaxis: a multicentre study

S. Jones, MD, MHSc, J. Bastian, MD, A. McRae, MD, D. Wang, PhD, K. Lonergan, BSc, E. Lang, MD; University of Calgary

Keywords: anaphylaxis, emergency medicine, gender differences Introduction: A number of mechanisms have been postulated to explain gender-specific differences in both the manifestations and incidence of anaphylaxis. However, no large-scale, population-based studies have examined the gender-specific incidence, recurrence, and management of anaphylaxis in the emergency department (ED). The purpose of our study is to explore gender-based variations in anaphylaxis, observed in a population of over 1 million urban inhabitants over 5 years. Methods: We conducted a retrospective cohort study in Calgary, Alberta. Data were drawn from administrative and electronic health record-derived sources and compiled between 2007 and 2011 from three tertiary care EDs. Inclusion criteria included age greater than 18, presentation to Calgary-area EDs, and a primary diagnosis of anaphylaxis determined by the International Classification of Diseases, Tenth Revision, and coded by a nosologist. Chi-square, Fisher exact, and Mann-Whitney $U$ tests were used to test the difference. Results: Anaphylaxis was diagnosed in 1,260 patients who presented to the ED over this period, of whom $8 \%$ were in the most acute triage category and 3\% were admitted to hospital. Patients who presented with anaphylaxis were more likely to be female $(55.4 \%$ v. $44.6 \% ; p<0.01)$, and females experienced longer delays to administration of their first dose of epinephrine (37 minutes v. 28 minutes; $p<0.05$ ). Male patients demonstrated higher acuity presentations than females $(85.2 \%$ v. $81 \% ; p<0.05)$ and more peanut-specific allergic reactions $(22.2 \%$ v. $16.5 \% ; p=0.01)$. No gender-specific differences were found for age at presentation, mode of arrival, medical management, length of stay, admission, or recurrence rates. Conclusion: Our results confirm that patients presenting with anaphylaxis are more likely to be female and challenge the belief that females have higher recurrence rates. They also highlight the delay in females receiving epinephrine, which could impact patient outcomes. This gender-specific variation in care could be explained by the difference in severity of presentations between female and male patients. 
P04 Reevaluating epinephrine in out-of-hospital cardiac arrest: a systematic review and meta-analysis

S. Lin, MDCM, MSc, C.W. Callaway, MD, PhD, P.S. Shah, MBBS, MSc, J.D. Wagner, BSc(c), J. Beyene, PhD, L. Morrison, MSc, MD, FRCPC; Division of Emergency Medicine, Department of Medicine, University of Toronto

Keywords: cardiac arrest, emergency medicine, epinephrine, metaanalysis

Introduction: International resuscitation guidelines support the routine administration of epinephrine during out-of-hospital cardiac arrest (OHCA). However, the evidence is not clear for the efficacy of epinephrine in long- and short-term survival. The objective of this study was to evaluate the efficacy of epinephrine (all doses and combinations) in adult OHCA patients on long-term (survival to discharge) and short-term (survival to admission or return of spontaneous circulation [ROSC]) outcomes. Methods: The search included MEDLINE, EMBASE, and the Cochrane Library up to March 1, 2012, and hand searches of bibliographies and electronic resources to identify eligible published and unpublished randomized controlled trials (RCTs). Eligible trials compared epinephrine to vasopressin or placebo in adult OHCA patients. Two independent reviewers conducted the hierarchical selection, abstracted data, and assessed quality. Disagreement was resolved by consensus. The Mantel-Haenszel random effects method was used to test for differences. A subgroup analysis was performed using stratification by location (prehospital or emergency department). Results: Fourteen RCTs $(N=12,246)$ met inclusion criteria: six compared standard-dose epinephrine (SDE) to $\geq 5$ times higher-dose epinephrine (HDE) $(n=6,174)$, six compared SDE to epinephrine vasopressin combination $(n=5,202)$, one compared SDE to vasopressin alone $(n=336)$, and one compared SDE to placebo $(n=534)$. There was no survival to discharge advantage in any of the comparison groups. HDE showed improved survival to admission (RR 1.15 [95\% CI: $1.00-1.32$ ], $p=0.05$ ) and ROSC (RR 1.17 [95\% CI: 1.03-1.34], $p=0.02$ ) over SDE. SDE showed improved survival to admission (RR 1.95 [95\% CI: 1.34-2.84], $p<0.001$ ) and ROSC (RR 2.80 [95\% CI: $1.78-4.41], p<0.001)$ over placebo in the single RCT. HDE and epinephrine vasopressin combination improved survival to admission when stratified by prehospital setting (RR 1.15 [95\% CI: 1.00-1.32], $p=0.05$ ) and emergency department (RR 1.20 [95\% CI: 1.04-1.38], $p=0.01$ ), respectively. Conclusion: There was no clear advantage of any vasopressor in long-term survival. Similar short-term benefit was seen with HDE over SDE and SDE over placebo, suggesting that the optimal dose of epinephrine remains unclear.

P05 Relationship between Canadian medical student identification of emergency medicine as a career choice and postgraduate training disposition

R. Abu-Laban, MD, MHSc, I.M. Scott, MD, MSc, M.C. Gowans, MSc; Department of Emergency Medicine, University of British Columbia

Keywords: emergency medicine certification, emergency medicine training, medical student

Introduction: Canada is unique in having two routes of emergency medicine (EM) training and certification overseen by independent colleges. Concerns have been raised that this system creates confusion for medical students and may encourage those with EM career aspirations to apply to family medicine (FM) residencies to subsequently acquire College of Family Physicians of Canada (CFPC) R3 training and certification of special competence in EM. We sought to determine whether medical students who indicated that EM was their top career choice on medical school entry but completed a family medicine (FM) residency after medical school were more likely than other FM residents to under- take subsequent CFPC-EM training. Methods: Data were accessed from a survey of medical students in 11 medical school classes from 8 Canadian universities carried out between 2002 and 2004 and anonymously linked to residency match information from the Canadian Residency Matching Service. Descriptive statistics were generated, and the chi-square test was used to compare proportions of medical students applying for CFPC-EM training in the two aforementioned groups. Research ethics board approval was obtained. Results: Of 1,349 eligible medical students, 1,036 (76.8\%) participated, 63 (6.1\%) of whom named EM as their top career choice on medical school entry. Of these, $10(15.9 \%)$ ultimately applied for and $8(12.7 \%)$ matched to a Royal College of Physicians and Surgeons of Canada (RCPSC) EM residency program, and $21(33.3 \%)$ applied for and $24(38.1 \%)$ matched to a FM residency program. Of the $380(36.7 \%)$ medical students who matched to FM residency programs, 9 of the 24 who had indicated EM was their top career choice on medical school entry went on to do a 1-year CFPCEM residency program in contrast to 57 of the remaining 356 medical students $(37.5 \%$ v. $16.0 \%, p=0.007)$. Conclusion: A significantly higher proportion of medical students with an early career interest in EM who go on to do an FM residency undertake CFPC-EM training compared to other FM residents. This finding, when considered in the wider context of the evidence and ongoing debate on whether or not changes to the Canadian system of EM training and certification are warranted, has potential implications that should be considered by both the RCPSC and the CFPC.

\section{P06 Limited access to services in Canadian rural emergency departments}

R. Fleet, MD, PhD, J. Villa, MPS, P.M. Archambault, MSc, MD, FRCPC, J. Poitras, MD; Université Laval and CHAU Hôtel Dieu de Lévis Hospital

Keywords: access to care, emergency medicine, health care system, rural emergency medicine

Introduction: Emergency departments (EDs) are important safety nets for $20 \%$ of Canadians who live rurally. Information on the services provided in rural EDs is scarce. Access to care is a cardinal feature of the Canada Health Act, yet recent efforts at cost containment through regionalization may impact access to comprehensive emergency care. Our objective was to examine access to services in rural EDs. Methods: This descriptive study uses mixed methods (interviews and database analysis). All EDs located in Canadian rural small towns (defined by Stats Canada) from each province/territory were selected to participate. We focused on hospitals with 24/7 ED physician coverage that have hospitalization beds. Data were collected from ministries of health, local health authorities, and ED statistics. A semistructured recorded phone interview was conducted with ED managers to collect additional data and confirm the status of services. Results: Among the 332 rural EDs identified, 329 (99\%) consented to participate. Hospitals had on average 22 acute care beds and 6 ED stretchers and averaged 12,200 annual ED visits. The proportion of rural hospitals having local access to the following $24 / 7$ services was intensive care unit $24 \%$, general surgeon $27.5 \%$, internal medicine $13 \%$, obstetrician $12.5 \%$, pediatrician $6 \%$, psychiatrist $9 \%$, CT scanner $15 \%$, ultrasound $22 \%$, and basic $\mathrm{x}$-ray and laboratory services $95 \%$. The average distance to the nearest referral hospital and trauma centre was about $240 \mathrm{~km}$. Conclusion: This is the first study to describe the services offered in all Canadian rural EDs. Other than basic laboratory and $x$-ray services, the majority of rural EDs have limited access to professional and ancillary services. A detailed study is required to evaluate the impact of these limited services on interfacility transfers, costs, professional recruitment/retention, and patient outcomes. Further analyses are required for interprovincial and rural-urban comparisons. 
P07 Correlation between venous and arterial blood gas samples in emergency department patients

A.A. Alrajhi, MD, MBA, FRCPC, A. Alreesi, MBBS, FRCPC, MHs, N. Alsayari, MD, A. Cluntun, MD; King Faisal Specialist Hospital \& Research Centre

Keywords: blood gases, emergency medicine, $\mathrm{pH}$

Introduction: Arterial blood gas (ABG) analysis is a frequently used test in the emergency department to assess acidosis and tissue oxygenation. A less invasive and painful venous blood gas (VBG) can be a good alternative. We sought to examine the agreement between $\mathrm{ABG}$ and VBG samples for $\mathrm{pH}, \mathrm{HCO}_{3}$, and lactate in emergency department patients. Methods: A prospective diagnostic study was conducted among patients presenting to a tertiary care emergency department over 18 consecutive months. After obtaining an informed consent, all patients who required $\mathrm{ABG}$ had VBG obtained at the same time; samples were analyzed independently using the same machine. The agreement between $\mathrm{ABG}$ and $\mathrm{VBG} \mathrm{pH}, \mathrm{HCO}_{3}$, and lactate was calculated using the Bland-Altman test with $95 \%$ CI. Results: A total of 190 patients were enrolled in the study; the mean age was 54.4 years (SD 1.5). The mean difference between arterial and venous $\mathrm{pH}$ was 0.032 (CI 0.026 to 0.038 ) with limits of agreement of -0.049 to 0.113 . The mean difference between arterial and venous $\mathrm{HCO}_{3}$ was -0.409 (CI 0.772 to -0.046 ), with limits of agreement of -5.488 to 4.67 . The mean difference between venous and arterial lactate was -0.39 (CI -0.473 to -0.310 ), with limits of agreement of -1.527 to 0.744 . Conclusion: Venous $\mathrm{pH}, \mathrm{HCO}_{3}$, and lactate are comparable to arterial ones and can be used as an alternative in patients seen in the emergency department.

P08 The current use of, perceived benefits of, and barriers to implementation of advanced ultrasound in Canadian emergency departments

D. Barbic, MD, M.S. Aldeeb, MBBS, S. Barbic, MSc, A. PihaGossack, BSc, J. Dankoff, MD; McGill University

Keywords: emergency medicine, knowledge translation, ultrasound Introduction: The use of advanced ultrasound (AUS) in the emergency department (ED) is limited, despite strong evidence supporting its use as a diagnostic aid and procedural guide in the ED. The objectives of this study were to summarize (1) current AUS use in Canadian EDs, (2) the perceived benefits of AUS use for daily clinical practice, and (3) barriers to AUS use in Canadian EDs. Methods: Structured telephone interviews were conducted by a trained research assistant using the Diffusion of Innovations model for knowledge translation (KT). A grounded theory approach was used to construct a substantive theory of barriers to AUS use in the ED. The sampling frame included Canadian attending emergency physicians (EPs). Results: Twenty-one EPs from five Canadian provinces participated. Eighty-one percent were male, $15 \%$ were from community hospitals, all EPs had completed a basic ultrasound course, and $43 \%$ had completed an AUS course. EPs described common use of AUS in procedural guidance (i.e., central venous access), assessment of IVC/fluid status, cardiac function, and pulmonary pathology. Perceived benefits included marked improvements in diagnostic accuracy, patient flow, and patient safety (procedural guidance). Identified barriers to increased AUS use in the ED included time required for training and practice, demands of clinical duties, and lack of quality assurance. Conclusion: Canadian EPs are using AUS in daily practice and perceive improved diagnostic accuracy, patient flow, and safety as significant advantages of its use. Training, clinical efficiency, and quality assurance were identified as key barriers to AUS use. Further training of Canadian EPs in these techniques and future knowledge translation on the potential benefits of AUS may be needed to promote utilization by more Canadian EPs.
P09 Evaluating a patient scheduling system in the emergency department using computer simulation modeling techniques H. Galbraith, MSc, D.W. Savage, PhD, A. Shearing, MD, M. Mensour, MD; Northern Ontario School of Medicine

Keywords: computer simulation, emergency medicine, patient scheduling

Introduction: In the summer months, the population of the Muskoka region of Ontario increases greatly with vacationers. The periodic rise in population poses a challenge for the emergency departments (ED) in the region because of increased patient arrivals, which have an impact on wait times and length of stay. We are investigating the impact of using a patient scheduling system in the ED for nonemergent patients. Interventions to increase efficiency in the ED typically examine throughput strategies; however, this study will attempt to modify the input or arrival rate of patients to reduce wait times and length of stay. Methods: We are using patient arrival, physician initial assessment, and departure data to develop parametric distribution models. These models will then be integrated into a discrete event computer simulation model that represents the major processes in the ED. Once the model is validated against actual ED data, it will be modified and used in an experimental framework to evaluate different patient arrival rates and levels of patient utilization. The primary model outputs will be wait time and length of stay in the ED. Results: The two primary output measures from the model are patient wait time and length of stay. These two measures are being used to compare the performance of the patient scheduling system. Since the model will be run multiple times, we will be able to estimate confidence intervals for the performance measures of interest. Conclusion: The model will provide evidence under which conditions the patient scheduling system performs best and should indicate whether this intervention in the Muskoka region would be beneficial for increasing patient flow in the ED during the summer months.

\section{P10 Socioeconomic status and the utilization of CT in the emer-} gency department

R. Bhayana, BSc, M. Vermeulen, MSc, Q. Li, MSc, C. Hellings, MSc, C. Berdahl, MD, M. Schull, MD, MSc; Division of Emergency Medicine (Department of Medicine), University of Toronto

Keywords: CT utilization, emergency medicine, socioeconomic status Introduction: Low socioeconomic status (SES) is associated with adverse health outcomes, potentially due to worse health status, differential access to health care, and differences in care provided by clinicians. The objective of this study is to determine whether SES is associated with computed tomography (CT) utilization in the emergency department (ED). Methods: We conducted a retrospective cohort study of patients presenting to EDs using administrative databases in Ontario. SES quintile was assigned based on median neighbourhood income. CT utilization was compared by SES quintile overall and in chief complaint subgroups (headache, abdominal pain, complex abdominal pain [age $>=65$, CTAS $1-3$, and admitted]) using multivariate logistical regression. Results: In adjusted analyses, higher SES patients were more likely to undergo CT imaging overall and in all clinical subgroups, with the exception of complex abdominal pain. Compared to the lowest SES quintile, the odds (aOR) of undergoing a CT scan in the highest SES quintile were 1.08 (95\% CI 1.07-1.09), 1.28 (95\% CI $1.22-1.34)$, and 1.24 (95\% CI 1.21-1.27) for all patients, headache, and abdominal pain patients, respectively. For patients presenting with complex abdominal pain, no difference in CT utilization was observed between the highest and lowest SES quintiles (aOR 1.09, 95\% CI 0.97-1.20). Conclusion: Lower SES is associated with lower utilization of CT scans in the ED overall and in patients with headache 
and abdominal pain. No difference was seen among complex abdominal pain patients, suggesting that as clinical indications for CT become more clear-cut, utilization across SES quintiles differs less. These results may indicate that some of the differential access to diagnostic testing among low SES patients reflects physician decision making.

\section{P11 Impact of an addiction assessment knowledge translation} strategy on physician attitudes and assessment behaviour

G. Salvalaggio, MD, MSc, CCFP, K. Dong, MD, MSc, FRCP, C. Vandenberghe, MSc, S. Kirkland, BSc, MSc, G. Cummings, PhD, R. McKim, MSc, R. Cooper, MD, MPH, FRCP, T. Wild, PhD; Department of Emergency Medicine, Faculty of Medicine \& Dentistry, University of Alberta

Keywords: addiction, emergency medicine, knowledge translation Introduction: Screening, brief intervention, and referral for treatment (SBIRT) in the emergency department (ED) can reduce problematic substance use; however, implementation has been suboptimal. The objective of this project was to develop and evaluate SBIRT knowledge translation (KT) resources designed to enhance physicians' capacity to intervene with low-socioeconomic position patients with substance use problems. Methods: This study used a nonrandomized, two-group, pre-post intervention design. Participants included ED and family medicine physicians and residents. Intervention physicians were offered accredited didactic presentations, facilitated small-group practice sessions, point-of-care resources, addiction medicine champions, and orientations to the inner city. Self-reported frequency of SBIRT use and physician attitudes (Short Understanding of Substance Abuse Scale [SUSS] and Attitudes Towards Injection Drug Use Scale [ATIDU]) were measured at baseline and 12 months. Comparisons between groups were done using mixed-design ANOVAs. Results: A total of 131 physicians participated and were allocated to intervention (78) or control (53) condition according to usual location of practice. Thirty-nine (50\%) intervention and $25(47 \%)$ control physicians were available for follow-up. Uptake of KT resources ranged from $29.7 \%$ for inner-city tours to $63.2 \%$ for point-of-care reminders; when used, the majority found these resources useful. Self-reported frequency of SBIRT behaviour did not differ between groups $(p=0.09)$, although there was a trend toward increased use among intervention ED physicians $(p=0.06)$. There were no significant differences in physician attitudes on the ATIDU $(p=0.06)$; however, there were favourable attitudinal changes on the SUSS Eclectic Orientation $(p<0.01)$ and Disease $(p<0.05)$ subscales. ED physicians also felt more successful in finding common ground with their socioeconomically disadvantaged patients $(p<0.05)$. Conclusion: A multipronged SBIRT KT strategy was favourably received by physicians and was associated with a trend toward improved attitudes toward patients with substance use issues; however, self-reported addiction assessment behaviour did not change. Further efforts should continue to explore ways to improve physicians' abilities to screen and intervene in substance-using patients.

P12 The socioeconomic composition of low-acuity emergency department users in Ontario

J.M. Caudle, MD, N. VanStone, PhD, P. Belanger, PhD, K. Moore, MD, MPH; Department of Emergency Medicine, Queen's University

Keywords: determinants of health, emergency medicine, triage acuity scores

Introduction: Emergency department (ED) crowding and lengthy wait times are an ongoing concern in Ontario. We describe the associations between the socioeconomic status (SES) of ED users and age, sex, and acuity of medical condition to better understand their common characteristics and to better meet primary and ambulatory health care needs. Methods: This is a retrospective, observational, population-based analysis of all Ontario ED visits for 2008 from the National Ambulatory Care Reporting System (NACRS) data set. We apply a rigorous proxy of SES using census-based methods to calculate a relative deprivation index (DI). ED visits are ranked into deprivation quintiles and associations between deprivation and age, sex, acuity at triage, and association with a primary care physician are investigated. Results: Over $25 \%$ of ED visits in Ontario are from the most deprived population; almost half of those (12.3\%) are for conditions of low acuity. Age profiles indicate that a large contribution to low-acuity ED visits are made by young adults (aged 20 to 30 years) from the most deprived population. For the highest-volume ED in Ontario, 94 of the 499 ED visits per day were for low-acuity patients from the most deprived population. Most of the highest-volume EDs in Ontario (over 200 ED visits per day) follow this general trend. Conclusion: Overall input into EDs may be reduced by providing accessible and appropriate primary health care resources in catchment areas of EDs with high rates of low-acuity ED visits, particularly for young adults from the most deprived population.

\section{P13 Level of patient satisfaction with the NP role in fast track: preliminary findings}

G. Martin, RN MN ENC, M. McGowan, MHK, M. Postic, MN, K. Gaunt, MHSc ENC; St. Michael's Hospital

Keywords: emergency medicine, fast track, nurse practitioner, patient satisfaction

Introduction: Nurse practitioners (NP) are registered nurses in the extended class ( $\mathrm{RN}[\mathrm{EC}])$ that order and interpret diagnostic tests, prescribe pharmaceuticals, perform procedures, and diagnose according to their legislated authority. There is growing evidence that NPs can provide complementary care to the traditional physician model, and research is needed as to the patient's experience with NPs in the ED. Methods: In an academic, inner-city, level 1 trauma centre (annual ED volume 72,000 ), an 18-question survey was designed by an interprofessional team to assess patient experience. All patients triaged to the fast track (FT) area of the ED were eligible and were provided with a letter of information and survey upon arrival at the clinical area. Clinicaldemographic and administrative data were collected from the ED clinical database. Results: Over a 4-week period, 140 surveys (45.6\% response rate) were completed, and respondents were representative (mean [SD] age $43 \pm 16.4$ years, $60.7 \%$ identified as male, $87 \%$ housed, $9.4 \%$ sheltered, $3.6 \%$ NFA) of the ED inner-city demographics. All were triaged CTAS $4(70 \%), 3(14.3 \%)$, or $5(12.9 \%)$, and delivery of care was efficient and timely: (1) time from triage to NP assessment 0:25 \pm 0:18 hr; (2) NP assessment to disposition 0:53 \pm 1:09 hr; and (3) overall ED LOS (triage to discharge) 1:22 $\pm 1: 29 \mathrm{hr}$. Patients appraised their experience with NPs as very good: thoroughness $(88.6 \%)$; listening $(90 \%)$; put at ease $(82.9 \%)$; involvement in decisions $(81.4 \%)$; explanation of the problem $(80 \%)$; and time spent with the patient $(81.4 \%)$. Following the NP visit, patients reported (1) understanding the health problem more (48.6\% agree, $37.1 \%$ strongly agree); (2) being able to cope more than before (51.4\% agree, $37.1 \%$ strongly agree); and (3) feeling the NP understood their health concern (55\% strongly agree, $40.7 \%$ agree). Overall level of satisfaction with care provided by the NP was high (67\% strongly agree, $30 \%$ agree); however, when asked if they would seek further care, there was a disparity $(40.7 \%$ agree or strongly agree, $36 \%$ strongly disagree or disagree, $18 \%$ undecided) with reported level of satisfaction. Of note, $72.9 \%$ reported having a regular family physician (FP). Conclusion: In a single site, preliminary patient-level satisfaction was found to be very high with NP care in the ED. Further investigations are warranted to assess the discordance between seeking further care and being satisfied with the care provided by the NP and why patients (CTAS 4,5 ) with a FP present to the ED. 
P14 Does prehospital continuous positive airway pressure impact the rate of intubation and mortality of acute respiratory emergencies?

S. Cheskes, MD, CCFP(EM), FCFP, L. Turner, PhD, S. Thomson, ACP, N. Aljerian, MD; Rescu, Li Ka Shing Knowledge Institute, St. Michael's Hospital

Keywords: continuous positive airway pressure, emergency medical services, emergency medicine, paramedic

Introduction: Previous small studies have demonstrated decreased rates of intubation and mortality with prehospital use of continuous positive airway pressure (CPAP). We sought to validate these findings in a larger observational study. Methods: We conducted an observational study of patients transported by EMS during the 12 months before and the 12 months following implementation of a prehospital CPAP protocol for acute respiratory distress. Included were all patients transported by EMS meeting pre-established criteria indicative of acute respiratory distress and CPAP use. Data were abstracted from ambulance call reports (ACRs) and hospital records. All cases in which a DNR was documented on the patient chart or ACR or whose inhospital outcome (death or discharge) was unknown were excluded. Results: In all, 442 patients met the above criteria. The mean (SD) age was 73.0 (13.9) years. In-hospital mortality rates did not differ for these patients: $17 / 228(7.5 \%)$ in the "before" group and 17/214 (7.9\%) in the "after" group ( $p=.72$, beta .60 to find an absolute difference of $6 \%)$. An analysis of the subgroup that had a hospital diagnosis of COPD, CHF, or pulmonary edema $(n=273)$ showed that mortality was lower in the "before" group $(3 / 138,2.2 \%)$ than in the "after" group $(8 / 135,5.9 \%)$ (Fisher exact test, $p=0.13)$. No patients in either group were intubated in the prehospital setting, and in-hospital intubation rates were similar for both groups $(11.6 \% \mathrm{v} .9 .7 \% ; \chi 2=0.26, p=$ $0.61)$. Conclusion: In contrast to previous studies, we were unable to demonstrate either a decrease in intubation or mortality related to the use of prehospital CPAP. Our findings may be specific to our EMS system but suggest that further large-scale randomized controlled trials may be warranted to firmly establish the benefit of prehospital CPAP.

\section{P15 Data gathering in resuscitation scenarios: novice versus expert physicians}

J.B. Baylis, BSc, S.M. Fernando, MSc, A. Szulewski, MD, D.W Howes, MD; Departments of Emergency Medicine and Critical Care, Queen's University

Keywords: crisis decision making, emergency medicine, resuscitation Introduction: Trauma and resuscitation medicine involve a series of calculated decisions made in succession in high-stress situations. The ability to quickly and efficiently gather data to make those decisions is of paramount importance. Recent research shows that experts tend to have different fixation and dwell patterns than novices, as well as a much more efficient search strategy in medical scenarios. The purpose of this study was to examine differences in information gathering between expert and novice resuscitation leaders. Methods: Four PGY5 and three PGY-1 physicians in the RCPSC Emergency Medicine program at Queen's University were enrolled in this pilot study. They wore Tobii eye tracking glasses while sequentially viewing three photographs of resuscitation scenarios for 10 seconds each. Following the viewing, participants were asked to describe what they observed in the scenario and what their next steps in management would be. Gaze sequence and fixation time were the primary outcomes. Results: Preliminary results showed that novice physicians spend more time looking around the scenario with shorter fixation times, while expert physicians focus more on particular areas such as the patient's face and the vitals monitor with longer fixation times. Also, expert physicians generally ordered fewer investigations in the postscenario interview while seeking specific information prior to further decision making. Conclusion: As a pilot study, this provides intriguing clues into how information gathering changes over the course of a physician's training. Experts appear to seek specific information in a crisis situation. Novices attempt to take in everything, possibly because they are unable to prioritize information. Experts may use more pattern recognition in resuscitation scenarios compared to novices. This research method offers exciting potential in accelerating the progression from novice to expert.

\section{P16 Tiered response system feasibility of anaphylaxis treatment} by fire first responders

M.J. Romano, MD, S. Cheskes, MD, R. Verbeek, MD, L. Turner, PhD; Sunnybrook Centre for Prehospital Medicine, Sunnybrook Health Science Centre, University of Toronto

Keywords: anaphylaxis, emergency medicine, feasibility, fire first responders

Introduction: Few studies have explored the potential for firefighter first responders to carry and administer epinephrine in cases of known anaphylaxis. We sought to explore the feasibility of this approach in a Canadian tiered response emergency medical system (EMS). The objective of this study was to determine the opportunity and feasibility to intervene by firefighter first responders in cases of anaphylaxis in a tiered response EMS system. Methods: This was a retrospective analysis of the ambulance call reports and fire services computeraided dispatch reports pertaining to all patients coded by paramedics as having received epinephrine for anaphylaxis by EMS in Peel Region from January 1, 2011, through November 30, 2011. For patients with a previous history of anaphylaxis, we deemed that an opportunity to intervene existed if firefighter first responders arrived at the patient's side greater than 2 minutes before paramedics. We established a priori that it was feasible in our tiered response system for firefighter first responders to intervene if this occurred in greater than $1 \%$ of tiered cases for anaphylaxis. Results: During the study duration, there were 199 cases of EMS-administered epinephrine for anaphylaxis. Of these, 51 cases $(25.6 \%)$ had firefighter first responders arriving prior to EMS, of which 22 met the criteria of previous history of anaphylaxis. Of these 22 cases, $12(54.5 \%)$ had fire first responders arriving at the patient's side a minimum of 2 minutes prior to EMS arrival. The overall opportunity for firefighter first responders to intervene was $12 / 199$ or $6.0 \%(95 \%$ CI $2.7-9.3)$, which met our a priori definition of feasibility. Conclusion: Using our a priori definition of feasibility, we established that in this tiered response EMS system, sufficient opportunity to intervene exisits to support firefighter first responders carrying and administering epinephrine for patients with a known history of anapyhlaxis. Further study is warranted to determine whether these findings are generalizable to other EMS systems and the safety of epinephrine administration by firefighter first responders for patients suffering from anaphylaxis.

\section{P17 Acetaminophen elixir pharmacokinetics in children aged 2 to 3 years}

D.A. Abourbih, BSc, MSc, E. Villeneuve, PharmD, S. Gosselin, MD; McGill University, Faculty of Medicine

Keywords: acetaminophen, emergency medicine, pharmacokinetics, toxicology

Introduction: Acetaminophen (APAP) elixir $(10-15 \mathrm{mg} / \mathrm{kg})$ is an acceptable pediatric antipyretic. A recent study by Kazim (2012) found that $30 \%$ of febrile children presenting to a pediatric emergency department received inadequate APAP dosages at home, with errors primarily due to age-based dosing. Parental education material in the form of weight-based dosing guides have been proposed; however, 
validation of current recommended APAP dosages using pharmacokinetic models is needed. An accepted model of APAP absorption will be used to compare the predicted plasma concentrations to the range required to achieve antipyresis $(10-20 \mu \mathrm{g} / \mathrm{mL})$. Methods: A common APAP preparation (Children's Tylenol Elixir ${ }^{\mathrm{TM}}$ ) was tested (children aged 2-3 years, 10.9-15.9 kg). The manufacturer's suggested dose of $160 \mathrm{mg}$ was compared to the standard 10 to $15 \mathrm{mg} / \mathrm{kg}$ dose. The model is represented below:

$$
\mathrm{Cp}=(\mathrm{F} \times \mathrm{D} \times \mathrm{Ka}) /(\mathrm{V})(\mathrm{Ka}-\mathrm{Kel}) \times\left[\left(\mathrm{e}^{-\mathrm{Kel} \times \mathrm{Tp}}\right)-\left(\mathrm{e}^{-\mathrm{Ka} \times \mathrm{Tp}}\right)\right]
$$$$
\mathrm{Tp}=1 /(\mathrm{Ka}-\mathrm{Kel}) \times[\ln (\mathrm{Ka} / \mathrm{Kel})]
$$

where $\mathrm{Cp}=$ peak plasma concentration $(\mu \mathrm{g} / \mathrm{mL}), \mathrm{F}=$ bioavailability $(0.90), \mathrm{Ka}=$ absorption constant (three values tested: 2.168, 7.92, $15.24), \mathrm{V}=$ volume of distribution $(0.95 \mathrm{~L} / \mathrm{kg}), \mathrm{Kel}=$ elimination constant (0.41), Tp = time to $\mathrm{Cp}$ (hrs), and $\mathrm{D}=$ dose (mg). Results: The model predicts a Cp between 6.38 and $8.55 \mu \mathrm{g} / \mathrm{mL}$ for $10 \mathrm{mg} / \mathrm{kg}$ and 9.57 and $12.8 \mu \mathrm{g} / \mathrm{mL}$ for $15 \mathrm{mg} / \mathrm{kg}$. The suggested dose of $160 \mathrm{mg}$ was tested across the limits of the weight range $(10.9$ and $15.9 \mathrm{~kg})$. A Cp between 9.36 and $12.6 \mu \mathrm{g} / \mathrm{mL}$ was found for the $10.9 \mathrm{~kg}$ child and 6.42 and $8.61 \mu \mathrm{g} / \mathrm{mL}$ for the $15.9 \mathrm{~kg}$ child. Conclusion: The $10 \mathrm{mg} / \mathrm{kg}$ dose does not reach the plasma cutoff for antipyresis $(10-20 \mu \mathrm{g} / \mathrm{mL})$, while $15 \mathrm{mg} / \mathrm{kg}$ is adequate, assuming a larger Ka. The $160 \mathrm{mg}$ dose is effective but only in smaller children (i.e., $10.9 \mathrm{~kg}$ ). Individual differences in $\mathrm{F}, \mathrm{V}$, and $\mathrm{Ka} / \mathrm{Ke}$ undoubtedly exist, and future studies directly measuring plasma APAP concentration and pharmacokinetics are planned.

\section{P18 Evaluation of intranasal fentanyl for pediatric patients in the emergency department}

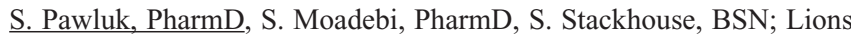
Gate Hospital

Keywords: emergency medicine, pain, pediatrics

Introduction: Acute pain is one of the most common symptoms of children presenting to emergency departments. Intranasal (IN) fentanyl offers an advantage when a short-acting agent is required and may prevent the need for intravenous administration. The objective was to characterize time to analgesia and identify safety parameters to monitor in children receiving IN fentanyl. Methods: A protocol for IN fentanyl ( $1.5 \mu \mathrm{g} / \mathrm{kg}$, followed by $0.5 \mu \mathrm{g} / \mathrm{kg}$ if pain persists after 10 minutes) for pediatric patients was developed, and demographic and pain data were collected using standard documentation. Time to analgesia, second dose of fentanyl given, change in vital signs, and side effects composed the primary outcomes. Nursing and physician staff satisfaction with the procedure was also documented. Results: A total of 105 pediatric patients who received IN fentanyl were included in the analysis. The median time to analgesia was 39 minutes (0-227 minutes). Median time to pain reduction by 2 or more points was 15 minutes, with $40(38.1 \%)$ patients requiring a second dose of IN fentanyl dosed at $0.5 \mathrm{mg} / \mathrm{kg}$. There were no significant changes in heart rate, respiratory rate, and oxygen saturations within 60 minutes after administration of IN fentanyl. IN fentanyl was overall very well tolerated. After administering IN fentanyl to these patients, both nurses and physicians could rank their overall satisfaction out of a total of 10 . The mean satisfaction score rated by the nursing staff was $8.5 \pm 2.3(n=53)$, and the mean satisfaction score rated by the physician staff was $8 \pm 2.8(n=20)$. Conclusion: These data demonstrate encouraging analgesic effects with IN fentanyl in children. The IN method of analgesic delivery did not cause any significant changes to heart rate, respiratory rate, and oxygen saturations and was well tolerated. These findings suggest that IN fentanyl provides an appropriate alternative for analgesia in children.

P19 Challenging medical dogma: a systematic review of peripheral vasopressor administration

O. Loubani, BSc, MD, R. Green, MD; QEII Health Sciences Centre
Keywords: complication, emergency medicine, peripheral intravenous, vasopressor

Introduction: Intravenously administered vasopressors are commonly used in circulatory shock. Tissue extravasation of these agents may cause local ischemia and necrosis due to their powerful vasoconstrictive effects. To avoid these complications, vasopressors are preferentially administered via central venous catheters. The evidence for this practice, however, is unclear. We conducted a systematic review to identify reported instances of complications, especially skin and tissue complications, arising from vasopressor administration via peripheral or central venous catheters. Methods: MEDLINE, EMBASE, and the Cochrane Controlled Trials Register were searched. No language or date restrictions were placed. Papers in which intravenous (IV) administration of one of 10 predetermined vasopressors or inotropes (dopamine, dobutamine, epinephrine, norepinephrine, milrinone, isoproterenol, vasopressin, terlipressin, phenylephrine, or ephedrine) resulted in one of our predetermined complications (neurologic, respiratory, cardiovascular, renal, gastrointestinal, dermatologic) were selected. References of selected papers and reviews on the subject were also searched. Results: Our search yielded 75,690 references. After title and abstract review, 520 full references were retrieved for review for final inclusion by both authors. In total, 114 references with 142 cases of complications resulting from vasopressor administration met final inclusion. There were 79 cases of skin and tissue complications from vasopressor administration, with 11 (14\%) resulting from central administration and 68 (86\%) from peripheral administration. In the 68 cases of skin and tissue complications from peripheral vasopressor administration, the vasopressor was administered in the foot most often (55\%) and for prolonged periods of time $(88 \%$ had vasopressor administration longer than 6 hours, with $69 \%$ longer than 24 hours). Norepinephrine was the vasopressor most commonly implicated in skin complications from peripheral administration (37/68, $54 \%$ of cases). Conclusion: Complications of peripheral IV administration of vasopressors have been infrequently reported in the literature. Skin and tissue complications resulting from peripheral vasopressor administration commonly involve prolonged administration of vasopressor into the foot. Further study is needed to clarify the safety of short-term vasopressor administration via peripheral IVs and its impact on outcomes.

\section{P20 Comparing access to services in rural emergency depart- ments in Ontario and Quebec: a pilot study}

R. Fleet, MD, PhD, C. Pelletier, BSc(C), J. Villa, MPS, L. Audette, MD, P.M. Archambault, MSc, MD, FRCPC, J. Poitras, MD; Université Laval and CHAU Hôtel Lieu de Lévis

Keywords: access to care, emergency medicine, health care system, rural emergency medicine

Introduction: Approximately $20 \%$ of Canada's population is rural. In the context of increasing budgetary constraints and health care professional shortages, a serious problem in access to quality emergency care in rural areas is forecast. Few studies have examined access to emergency services in rural Canada. With the purpose of planning a nationwide study, this pilot project's main objective was to compare Ontario $(\mathrm{ON})$ and Quebec (QC) with respect to access to support services to rural EDs. Methods: Rural EDs were identified with the Canadian Healthcare Facilities Guide; their status was confirmed by provincial authorities. We selected hospitals with $24 / 7$ ED physician coverage and hospitalization beds that were located in rural communities (rural and small town definition - Statistics Canada). A semistructured phone interview was conducted with ED managers/nurses of every ED to collect denominative statistics and confirm the status of available services. Interviews were audiotaped. Ten percent were randomly reviewed for protocol adherence verification. Results: The participation rate was 
$100 \%$. ON has more rural EDs $(n=62)$ than QC $(n=26)$. QC EDs have higher volumes (19,310 v. 13,180 annual visits). With respect to support services, $81 \%$ of QC's rural EDs have $24 / 7$ local access to a general surgeon compared to $33 \%$ for ON. Nearly $75 \%$ of QC rural EDs have a CT scan versus $12 \%$ for ON. Rural EDs in QC are also supported by a greater proportion of intensive care units ( $88 \%$ v. $31 \%)$. The average distance to the nearest tertiary trauma centre is greater in QC $(334 \mathrm{~km})$ versus ON $(220 \mathrm{~km})$. Conclusion: Preliminary results suggest that major differences exist in access to support services in rural EDs in ON and QC. Do ON EDs provide adequate levels of service? Does QC have too few yet well-equipped EDs? A nationwide study is required to understand this issue of variability and the potential impact on quality emergency care.

P21 Methicillin-resistant Staphylococcus aureus (MRSA) colonization among health care workers in a downtown emergency department in Toronto, Canada

C. Varner, MD, CCFP(EM), B. Borgundvaag, PhD, MD, A. McGeer, MD, FRCP; Mount Sinai Hospital

Keywords: emergency medicine, health care workers, MRSA

Introduction: Methicillin-resistant Staphylococcus aureus (MRSA) acquired in the community, otherwise known as community-acquired MRSA (CA-MRSA), has emerged rapidly in recent years. Colonization with MRSA has been associated with increased risk of symptomatic and serious infections, and in some settings, health care workers (HCWs) have a higher prevalence of MRSA colonization. The purpose of this study was to determine MRSA colonization in emergency department (ED) HCWs in the setting of a moderate prevalence of MRSA in skin and soft tissue infections (SSTIs). Methods: This study was conducted at a downtown ED in Toronto, Canada. ED HCWs completed a brief questionnaire, and swabs were taken from one anterior nare, one axilla, and any open wounds if present. Swabs were processed using standard laboratory techniques. Results: None of the 89 staff (registered nurses [ $n=55]$, physicians [ $n=15]$, other $[n=19]$ ) were MRSA positive and $25(28.1 \%)$ were colonized with MSSA. Conclusion: Contrary to common belief among HCWs and previous studies documenting MRSA colonization of HCWs, MRSA colonization of this Canadian ED HCW cohort is very low and similar to the local population.

P22 Reliability of clinical decision rules to improve the detection of adverse drug events in emergency department patients

C.M. Hohl, MDCM, FRCP, MHSc, L.N. Reddekopp, Bachelor of Nursing, E. Yu, MS, J. Rotinsky, Baccalaureate, C. Stang, Masters; University of British Columbia

Keywords: adverse drug event, clinical decision rule, emergency medicine, patient safety

Introduction: Adverse drug events (ADEs), unintended and harmful effects of medications, are often misdiagnosed in the emergency department (ED). We derived a clinical decision rule to identify patients at high risk for ADEs. Our objective was to determine the reliability of the component questions of the rule and of the rule's interpretation as a whole when applied in real time by triage nurses. Methods: In this prospective study, triage nurses were trained to apply and interpret a clinical decision rule containing three questions: (A) Have you taken medications within 2 weeks? (B) Do you have pre-existing medical problems, or have you been on antibiotics within 7 days? (C) Are you $>80$ years, or have you changed medications within 28 days? The research coordinator trained nurses to apply and interpret the rule in one lecture and subsequently provided one-on-one feedback if she identified incorrect flags. We programmed the computerized patient information systems to require input of the flag after 8 weeks and integrated electronic logic for the rule's interpretation at one site after 6 months. We measured the reliability of the component questions of the rule and its interpretation by comparing to a criterion standard derived by the study coordinator using kappa scores with $95 \%$ confidence intervals (95\% CIs). Results: We approached 812 patients, of whom 678 met inclusion criteria. The reliability of the component questions of the rule was $0.74(95 \% \mathrm{CI} 0.67-0.81)$ for question $\mathrm{A}, 0.78(95 \% \mathrm{CI}$ $0.69-0.88)$ for question $\mathrm{B}$, and $0.68(95 \%$ CI $0.60-0.76)$ for question C. The reliability of the nurses' interpretation of the rule was 0.74 (95\% CI 0.69-0.79) compared to the criterion standard. The reliability of the rule's interpretation was unchanged before and after implementation of electronic logic $(0.79 ; 95 \%$ CI $0.70-0.88$ pre versus 0.75 ; 95\% CI $0.63-0.88$ post). Conclusion: The reliability of the ADE rule when applied at triage in real time was high.

\section{P23 The Calgary multifaceted intervention for suspected appen- dicitis reduces ED length of stay and time to surgery \\ J. Gill, BSc, P. Lalli, BSc, D. Wang, PhD, L. Mercuur, MD, E. Lang, $\mathrm{MD}, \mathrm{A} . \mathrm{McRae}, \mathrm{MD}$; University of Calgary}

Keywords: administration, appendicitis, diagnostic imaging, emergency medicine

Introduction: Suspected acute appendicitis cases requires concise and accurate assessment to determine whether surgical intervention is needed and to avoid complications such as perforations, abscess formation, sepsis, and possibly death. We developed an Alvarado scorebased multifaceted intervention that included expedited imaging and increased ultrasound usage to decrease time to diagnosis and treatment of appendicitis.This time series design study evaluated the impact of this system-level intervention on time to diagnosis and surgery for acute appendicitis. Methods: The study enrolled patients admitted for acute appendicitis in three adult urban EDs either before (November 2008 to April 2009) or after (November 2011 to April 2012) the implementation of our protocol. Primary outcomes included median times for triage to surgical incision, triage to MD assessment, MD assessment to consultation, admission to surgical incision, and ED length of stay (LOS). Time interval values were compared using Mann-Whitney $U$ testing. Results: A total of 408 patients were included, with 184 patients managed using the "pre" protocol and 224 with the "post" protocol. Patient groups were similar with respect to age, gender, and Canadian Emergency Department Triage and Acuity Scale (CTAS). ED length of stay for appendicitis patients improved from the pre to the post period (median of 10.66 hours pre, 8.08 hours post, $p<0.001$ ). We also observed shorter LOS components in the post period, including time from triage to physician assessment (median 2.25 hours pre, 1.43 hours post, $p<0.001$ ), time from physician assessment to consultation (median 3.88 hours pre, 3.03 hours post, $p<0.05$ ), and time from consultation to admission decision (median 1.82 hours pre, 1.50 hours post, $p<0.05$ ). However, time from decision to admit until surgical incision increased (median of 3.12 hours pre, 3.89 hours post, $p<$ 0.05). Conclusion: The Calgary Protocol improved ED LOS and components of LOS of patients with appendicitis. However, further work is needed to improve delays from time of admission to time of surgical intervention and for overall ED length of stay.

P24 The impact of "casino shifts" on emergency physician productivity

A. Dukelow, MD, FRCPC, D. Thompson, MD, FRCPC, M. Peddle, MD, FRCPC, C. Rhiger, RN, M. Klingel, MSc, M. Lewell, MD, FRCPC, S.L. McLeod, MSc; The University of Western Ontario

Keywords: casino shifts, emergency medicine, productivity 
Introduction: Some emergency physician (EP) groups have changed their scheduling to include "casino" shifts over the past 10 years. Casino shifts usually change over at 0400 instead of the traditional 0700 or 0800 . The primary objective of this study was to determine the impact of a shorter casino shift on physician productivity. Secondary objectives were to compare the acuity of patients seen during 6-hour casino shifts and 8-hour night shifts and also to determine the number of patients seen during the first and last 2 hours of each type of night shift. Methods: This was a retrospective chart review of all patients arriving at one of two emergency departments (EDs) at a tertiary care centre (annual census 125,000) between February 1-28, 2012, March 15-30, 2012, and June 1-30, 2012, and seen by a nighttime EP. The most responsible physician (MRP) for each patient and the shift that the MRP was working were recorded. The total number of patients per hour seen, average Canadian Triage and Acuity Scale (CTAS) scores, and number of learners working an 8-hour nighttime shift (1800-0200 and 1900-0300) versus a 6-hour casino shift (2200-0400 and 04001000) were compared. Results: Fifty different EPs worked 148 8-hour nighttime shifts and 298 casino shifts and saw 7,958 patients during the study period. EPs saw a mean (SD) 2.4 (0.6) patients per hour on regular 8-hour night shifts and $2.9(0.7)$ patients per hour on 6-hour casino shifts $(\Delta 0.5,95 \% \mathrm{CI}: 0.4,0.7)$. The mean CTAS scores of patients seen on 8-hour nighttime shifts versus 6-hour casino shifts were equally distributed and statistically similar between both groups. EPs saw a mean (SD) 2.8 (1.0) patients per hour during the first 2 hours of a regular 8-hour night shift, compared to 3.9 (1.3) patients per hour during the first 2 hours of a 6-hour casino shift $(\Delta 1.1,95 \% \mathrm{CI}$ : $0.8,1.3)$. However, EPs saw a mean (SD) 2.1 (1.1) patients per hour during the last 2 hours of a regular 8-hour night shift, compared to 1.8 (1.2) patients per hour during the last 2 hours of a 6-hour casino shift $(\Delta-0.3,95 \%$ CI: $-0.03,-0.5)$. Conclusion: Six-hour casino shift scheduling may improve the number of patients seen during a night shift. Future studies should examine the impact of casino shifts on cognitive performance, patient safety, and EP satisfaction.

P25 The Canadian National EMS Research Agenda: a survey to determine the impact and feasibility of implementing the recommendations

J.L. Jensen, ACP MAHSR, I. Blanchard, EMT-P, MSc, B.L. Bigham, MSc ACP, A. Carter, MD, R. Brown, BSc PCP, D. Socha, BSc, PCP, L.H. Brown, PhD, MPH\&TM, A. Travers, MSc, MD, A. Craig, ACP MScPL, L. Morrison, MSc, MD, FRCPC; Dalhousie University, Division of EMS

Keywords: agenda, emergency medicine, implementation, research Introduction: A recent study on the state of EMS research in Canada generated 19 actionable recommendations. This survey objective was to determine the anticipated impact and feasibility of implementing these recommendations. Methods: An online survey explored the potential impact and feasibility (ease or difficulty of implementation) for each recommendation using 5-point Likert scales. The study sample consisted of participants from the EMS Research Agenda study (2012) and additional EMS research stakeholders identified through snowball sampling. Responses were analyzed descriptively using median and were plotted on a matrix (feasibility $\times$ impact). Participants reported any planned or ongoing initiatives related to the recommendations and required or anticipated resources. These free text responses were analyzed with simple content analysis, collated by recommendation. Results: The survey was sent to 131 people; 94 responded (71.8\% response rate), including EMS managers/regulators $(n=30$, $31.9 \%)$, researchers $(n=22,23.4 \%)$, physicians $(n=15,16.0 \%)$, educators $(n=13,13.8 \%)$, and EMS providers $(n=5,5.3 \%)$. Two recommendations had a median impact score of 4 (out of 5) and a feasibility score of 4 (out of 5 ). Three to four resources per recommendation were required. Eight recommendations had a high impact score of 5 and a lower feasibility score of 3 , with 33 ongoing or planned initiatives (range 1-9 per recommendation), and 30 resources identified (range 17 per recommendation). Nine had an impact score of 4 and a feasibility of 3 with 29 initiatives (range 2-5 per recommendation), and 34 resources required (range 2-11 per recommendation). Conclusion: For most recommendations, participants scored the anticipated impact higher than the feasibility to implement, and there are ongoing or planned initiatives pertaining to all recommendations except one, yet all require at least one additional resource to implement. The national implementation of EMS Research Agenda recommendations is anticipated to make important improvements in EMS research but perceived to require dedication, resources, and collaboration across planned and ongoing initiatives to achieve.

\section{P26 Can paramedics diagnose sepsis in the prehospital setting? A} feasibility study

A. Travers, MSc, MD, R. Green, MD, E. Cain, MD, FRCPC, S.G. Campbell, MB BCh, J.L. Jensen, ACP MAHSR, D. Petrie, MD, FRCPC; Dalhousie University, Division of EMS

Keywords: diagnosis, emergency medicine, paramedics, sepsis Introduction: Sepsis patients benefit from early goal-directed therapy (EGDT). Accurate paramedic diagnosis is important to initiate EGDT promptly. The objective was to evaluate diagnostic performance of sepsis by paramedics, compared to emergency physician (EP) diagnosis. Methods: This prospective observational study was of a convenience sample of adult patients transported to a tertiary emergency department (ED) by paramedics. Patients were enrolled if dispatched as abdominal pain, breathing problems, sick person, unknown problem, unconscious/fainting, chest pain, or any case in which paramedics considered sepsis a possible diagnosis. Paramedic diagnosis of sepsis were entered into study data collection forms, which were compared to blind, independent documentation of diagnosis by the attending EP (considered gold standard). For missing EP forms, ED chart review was conducted. Specificity, sensitivity, accuracy, positive and negative predictive value, and ratios were calculated with $95 \%$ confidence intervals. Results: A total of 956 patients were enrolled by paramedics between January and September $2008 ; 327 / 956(34.2 \%)$ excluded: no paramedic diagnosis recorded $(249 / 956,26.0 \%)$, no EP diagnosis available (73/956, $7.6 \%$ ), patient left ED without being seen/no EP diagnosis made $(5 / 956,0.5 \%)$. Paramedic and EP diagnosis was available for $629 / 956(65.8 \%)$ and were included in the final analysis. Paramedics identified 170/629 (27.0\%) patients as septic and EPs identified $71 / 629(11.3 \%)$. Sensitivity and specificity of paramedic sepsis diagnosis were $73.24 \%$ (95\% CI $61.40-83.05)$ and $78.85 \%(95 \% \mathrm{CI}$ 75.23-82.17), respectively. Accuracy was $78 \%(492 / 629,52$ true positive, 440 true negative). The positive and negative predictive values were $30.59 \%$ (95\% CI $23.76-38.11)$ and $95.86 \%$ (95\% CI 93.61-97.49), respectively. The positive likelihood ratio was 3.46 (95\% CI 2.80-4.29), and the negative likelihood ratio was 0.34 (95\% CI: 0.23-0.50). Conclusion: Paramedic diagnosis of sepsis has greater specificity than sensitivity, with reasonable accuracy. Further research should evaluate thermometry and point-of-care lactate testing diagnostic tools, derivation of a clinical predication rule, and EGDT delivery in the prehospital setting.

P27 Barriers, facilitators, recommendations, and priorities for EMS research: a scoping review of research agendas

I. Blanchard, EMT-P, MSc, R. Brown, BSc PCP, J.L. Jensen, ACP MAHSR, B.L. Bigham, MSc ACP, A. Travers, MSc, MD, A. Carter, MD, L. Morrison, MSc, MD, FRCPC; Dalhousie University 
Keywords: emergency medical services, emergency medicine, paramedic, research agenda

Introduction: Several countries and organizations have created research agendas in an effort to improve and focus the EMS (emergency medical services) research enterprise. To date, results from these agendas have not been compiled, which may hinder knowledge translation. Scoping reviews are used to map broad topics, summarize, and disseminate research findings. This methodology was used to map reported research agenda methods, barriers and facilitators to EMS research, recommendations made, and prioritize research topics/outcomes. Methods: A combination of MeSH and keywords were used to search MEDLINE, EMBASE, CINAHL, Google Scholar, and grey literature using Google. Search results were subject to two review rounds for inclusion: (1) title, weblink, and abstract and (2) full article screening. Articles were included if they were a knowledge-generating project where a group of national stakeholders in EMS research reached consensus on barriers, facilitators, recommendations, or prioritization of research topics/outcomes. Results: A total of 3,618 titles, weblinks, and abstracts and 52 full-text articles were reviewed. Ten distinct EMS research agendas reported in 19 articles were included, 15 articles from peer-reviewed journals and 4 from non-peerreviewed sources. Agendas from Australia $(n=1)$, Canada $(n=1)$, Europe $(n=1)$, Ireland $(n=1)$, UK $(n=1)$, and the USA $(n=5)$ used 13 unique methodologies to report 32 barriers and 29 facilitators to EMS research. Agendas proffered 65 recommendations for improving the research enterprise with some setting-specific implementation strategies, and 253 prioritized topics and outcomes. Conclusion: Multiple EMS research agendas were identified employing a variety of methods revealing barriers, recommendations, and priority research topics/outcomes, but few agendas reported facilitators. Translation of these results at the local level may be a starting point for actionable changes to the EMS research enterprise. Future research should quantify the impact that these agendas have had on improving the quality, quantity, and usefulness of EMS research.

\section{P28 Establishing priorities for patient safety research in the emer-} gency department

A. Plint, MD, A.S. Stang, MD, MBA, MSc, L.A. Calder, MD, MSc. FRCPC; University of Ottawa

Keywords: emergency medicine, patient safety, research priorities Introduction: Recognizing the importance of patient safety, the unique risks to patient safety in the emergency department (ED) setting, and the need for clear direction for future safety-related research, we report the results of a consensus process with the goal of developing an end user-driven list of safety-related research priorities for all patients seen in the ED. Methods: The process was led by a 19-member expert panel with broad representation of clinicians and researchers from adult emergency medicine, pediatric emergency medicine, patient safety, pharmacy, and mental health. We also had representation from knowledge synthesis experts, patient safety organizations, and health care administrators. To develop a comprehensive list of potential research priorities, we electronically surveyed our expert panel as well as patient safety experts, ED clinicians, and researchers from across North America. The survey asked respondents to list, without limitation, possible research priorities. Following the survey, duplicates were removed and suggested priorities were organized into themes. Consensus on final research priorities was obtained using a modified Delphi process through a further iterative survey of our expert panel and a facilitated in-person meeting. Results: Thirty-two (32.7\%) of 98 individuals surveyed for suggestions of research priorities responded. Overall, respondents suggested 117 research priorities; individual respondents suggested a mean of 3.7 priorities (range of $1-10$ ). After removal of duplicates, there were
62 unique priorities for expert panel review. Following our modified Delphi process and in-person, facilitated meeting, 15 research priorities remained. These priorities fall into four themes: (1) exploring methods to identify patient safety issues (five priorities), (2) understanding human and environmental factors related to patient safety (four priorities), (3) the patient perspective (one priority), and (4) interventions in patient safety (five priorities). Discussion at the inperson meeting also resulted in the identification of three guiding principles for all patient safety research in the emergency department. These three guiding principles are as follows: (1) to address these priorities, we need to build and sustain truly collaborative partnerships between clinicians and safety scientists; (2) when conducting research in patient safety, a clear identification and reporting of the taxonomy used are strongly recommended; and (3) innovative and novel methods outside the conventional clinical investigative techniques are required. Conclusion: This rigorous consensus process provides end user-driven research priorities for patient safety in the ED. The establishment of guiding principles and a stakeholderprioritized research agenda are a key step in improving patient safety in the high-risk ED setting.

\section{P29 CAEP IEM Committee: results of a national survey of inter-} national emergency medicine practitioners

C.L. Heslop, MD/PhD, C. Kowal, MD, CCFP(EM), R. Venugopal, MD, MPH, FRCPC; Emergency Medicine Residency Program, University of Toronto

Keywords: emergency medicine, global health, international emergency medicine

Introduction: The CAEP International Emergency Medicine (IEM) committee includes clinicians interested and involved in international emergency medicine. Project types and degrees of involvement vary significantly between members across Canada. Also, collaboration among members at different training at sites is currently unstructured as no Canadian database exists to describe projects across the nation. There are potential benefits to sharing information regarding projects in global health and international emergency medicine, both for clinicians launching or undertaking projects and for EM trainees interested in getting involved in global health. Methods: A comprehensive, Webbased survey was initially proposed during the CAEP IEM Committee meeting in June 2012. Following an extensive piloting and feedback gathering process, the survey was developed on a Web-based platform (Survey Gizmo). The survey was tested among IEM Committee members and emergency medicine trainees. Following feedback from the testing phase, the survey was distributed to IEM Committee members and to emergency clinicians and trainees at sites across Canada. Results: Surveys were collected, and information about IEM projects was compiled into a database. Survey results indicate a great deal of activity and a wide variety of projects going on across the country. Data regarding challenges and successes resulting from each clinicians' IEM experiences were collected and compiled for the purpose of supporting collaborations and improving outcomes of IEM projects Canada-wide. Final survey results will be reviewed in detail and also presented at the CAEP IEM Committee meeting panel. Conclusion: This survey of IEM practitioners has much to offer to Canada's global emergency medicine community. Trainees interested in participating in global emergency medicine will gain the opportunity to make contacts and join projects, and project leaders can share information about overcoming challenges inherent to IEM and increase their collaboration with IEM colleagues nationwide.

P30 Musculoskeletal education in emergency medicine residency: perceived needs of residents and program directors 
D. Hill, MD, A. Pozgay, MD, J.R. Frank, MD, MA(Ed), FRCPC, I.G. Stiell, MD, MSc, FRCPC; University of Manitoba, Department of Emergency Medicine

Keywords: education, emergency medicine, musculoskeletal Introduction: Musculoskeletal (MSK) complaints are a common presenting complaint to the ER; however, the quality of MSK teaching during the FRCP Emergency Medicine (EM) residency program is often questioned. The objective of this study was to assess the comfort level of FRCP-EM residents in dealing with MSK complaints, as well as to assess attitudes of residents and program directors (PDs) toward MSK education. Methods: We conducted a national electronic survey of FRCP-EM residents and PDs. Data were collected on demographics, program requirements, comfort level of residents in assessment, procedural skills, and disposition of MSK complaints, as well as attitudes toward the importance of MSK education and the need for an improved curriculum. Data were analyzed using simple descriptive statistics. Results: A total of 121 (37.5\%) residents and 14 (73.7\%) PDs answered the survey. Residents felt strongest in the assessment of ankle/foot pain and low back pain (75.2\% rated comfort level $\geq 4 / 5)$. Residents felt weakest in the assessment of hip/groin pain $(56.2 \%$ rated comfort level $\leq 3 / 5$ ). For procedural skills, residents felt most comfortable with shoulder reductions $(63.6 \%$ rated comfort level $\geq 4 / 5)$ and least comfortable with joint injection techniques $(78.5 \%$ rated comfort level $\leq 3 / 5$ ); $52.9 \%$ of residents rated their ability to make appropriate orthopedic referrals $\leq 3 / 5$. Overall, $75.2 \%$ of residents rated their MSK education in residency $\leq 3 / 5$, and $57.9 \%$ rated the need for an improved MSK curriculum $\geq 4 / 5$. For the PDs, $92.9 \%$ rated the importance of MSK education in FRCP-EM residency $\geq 4 / 5$, and $57.1 \%$ rated the need for an improved MSK curriculum $\geq 4 / 5$. Conclusion: FRCP-EM residents lack comfort in dealing with many MSK complaints. There is consensus among residents and PDs that MSK education is an important part of the residency program and that there is a need for an improved curriculum. EM educational leaders should consider urgent reforms to enhance training.

P31 The effect of age and sex on the proportion of patients with acute coronary syndrome (ACS) who present "typically" to the emergency department (ED)

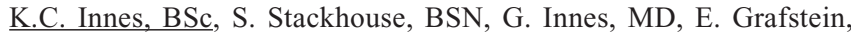
$\mathrm{MD}$; University of British Columbia

Keywords: acute coronary syndrome, emergency medicine, triage Introduction: Women and older patients may present with atypical ACS symptoms. Our objective was to determine the proportion of ACS patients, stratified by age and gender, who report "typical" ACS symptoms to ED triage nurses. Methods: This administrative database study was performed using prospectively collected data from six Vancouver Coastal Health EDs during a 6-month period in 2012. These sites use common complaint-linked triage codes and standardized triage training. Nurses are instructed to code patients with possible ACS into category chest pain $(\mathrm{CP})$ - cardiac features, but codes $\mathrm{CP}+$ respiratory distress, $\mathrm{CP}+$ hemodynamic compromise, and $\mathrm{CP}+$ ripping or tearing are also compatible with typical ACS presentations; therefore, these codes were considered typical. Patients with a discharge diagnosis of ACS (AMI, UA, angina) were included, and their ED triage codes were captured. Results: A total of 1,238 ACS patients were studied (806 male, 432 female), with a mean age of 68 years $(\mathrm{SD}=14)$. Of these, 817 had AMI, 340 had UA, and 81 had angina. Overall, 58.5\% fell into typical triage categories (M 61.8\%, F 52.6\%). Below age 40 ( $n=14 \mathrm{M}, 4 \mathrm{~F})$, $39 \%$ were typical (M 50\%; F 0\%). From 40 to 49 years ( $n=54$ M, 26 F), 65\% were typical (M 63\%, F 69\%). From 50 to 59 years $(n=169$ M, 60 F), 64\% were typical (M 62\%, F 70\%). From 60 to 69 years $(n=$ 229 M, 84 F), 59.1\% were typical (M 59.8\%, F 57.1\%). From 70 to 79 years ( $n=172 \mathrm{M}, 98 \mathrm{~F}), 63.7 \%$ were typical (M 69.8\%, F 53.1\%). From 80 to 89 years $(n=137 \mathrm{M}, 124 \mathrm{~F}), 52.9 \%$ were typical $(\mathrm{M}$ $57.7 \%$, F 47.6\%). Over age $90(n=31 \mathrm{M}, 36 \mathrm{~F}), 35.8 \%$ were typical (M $51.6 \%$, F 22.2\%). Conclusion: Overall, less than $60 \%$ of ACS patients had typical presentations. Atypical presentations increased in women over 60 and men over 80 . Gender differences were insignificant from age 40 to 70 . Most patients under 40 were triaged into atypical categories, but $n$ was small in this group. Triage nurses depending on traditional symptom descriptors will miss many patients with ACS.

P32 Development of a novel Web-based communication tool to improve emergency department discharge communication with family physicians: a needs assessment survey

C. Hunchak, MD, CCFP(EM), MPH, A. Patel, MD, CCFP, A. Teo, MD, CCFP, D. Tannenbaum, MD, CCFP, FCPC, M. Roberts, MD, CCFP, FCFP, H.J. Ovens, MD, B. Borgundvaag, PhD, MD; University of Toronto

Keywords: emergency care information systems, emergency medicine, medical informatics application

Introduction: Communication of emergency department (ED) visit information to family physicians (FP) is often inadequate and can negatively impact on patient care. Further, the use of email as a health communication tool between FP and ED providers has not been adequately explored. We sought to assess the desirability, feasibility, and ideal functionalities of a Web-based, automated post-ED visit communication tool for FPs. Methods: A cross-sectional needs assessment survey was conducted among the top 300 referring FPs to the Mount Sinai Hospital ED in Toronto, Canada. The main outcome measures were current FP awareness of patient ED visits and anticipated uptake of a Web-based post-ED visit notification and health records access communication tool. Results: A total of 198 of 300 physicians responded (66\% response rate). Fifty-eight percent of FPs were unaware or only sometimes aware when their patients had visited the ED. Nearly all (94\%) surveyed FPs would welcome a novel automated electronic system to communicate post-ED discharge health record information in real time. Two-thirds $(67 \%)$ of respondents were also in favour of their patients having online access to their own information. Physicians less than 50 years of age were significantly more likely than those greater than 50 years to use an office computer with Internet and email access $(96.4 \%$ v. $68.6 \%, p<0.001)$ and were significantly more likely to use EMR in their office $(57.1 \%$ v. $41.1 \%, p=0.04)$. Conclusion: This needs assessment survey highlights an unmet need for improved ED-FP health record communication and suggests that FP uptake of a secure Web-based notification tool to alert FPs of patient ED visits and to provide timely access to laboratory and diagnostic results would be high.

\section{P33 Critical dynamics: demographic influences on burnout in emergency department health}

M. Howlett, MD, K. Doody, MA, J. Murray, MD, D. Leblanc-Duchin, BSc, MA, PhD, J. Fraser, Bachelor of Nursing, P.R. Atkinson, MD FCEM; Discipline of Emergency Medicine, Memorial University

Keywords: burnout, emergency department, emergency medicine, health professionals

Introduction: Pressures for emergency department (ED) health professionals lead to burnout, as characterized by the Maslach Burnout Inventory (MBI). We examined demographic trends in EDHPs at seven emergency departments (ED) in New Brunswick to identify predictive factors for staff burnout. Methods: A total of 322 of 615 ED health professionals $(51 \%)$ completed an MBI (subscales Emotional Exhaustion, Depersonalization, and decreased Personal Accomplishment) and a demographics questionnaire. We examined baseline 
covariates and burnout scores using univariate and multivariate linear regression models. Results: Certified nurses had higher emotional exhaustion than those with other forms of certification $(p=0.009)$. Longer tenure in the same ED (compared to 5 years or less) up to 20 years was associated with higher emotional exhaustion $(p=0.029)$ and higher depersonalization $(p<0.0001), 6$ to 10 years; emotional exhaustion $(p<0.001)$ and depersonalization $(p=0.010), 10$ to 20 years. Working for more than 20 years had no effect on any MBI subscale. Professional stress was associated with higher emotional exhaustion $(p<0.001)$, but personal stress had no effect in any of the models. Males had higher personal accomplishment (Est. $=2.17, t=2.20, p=$ $0.029)$ while controlling for other variables. Conclusion: A relationship exists between burnout and longer tenure in the same ED, professional stress, and being a nurse by increasing the risk of emotional exhaustion. Tenure also increases depersonalization. Gender may influence one's sense of personal accomplishment.

\section{P34 Prehospital anaphylaxis mimics in one Canadian urban centre} T. Chung, BSc (Candidate), L. Lovstrom, BSc, MSc (Candidate), C. Vandenberghe, MSc, S. Couperthwaite, BSc, S. Sookram, MD, FRCPC, K. Liss, BSc, MBA, C. Villa-Roel, MD, PhD (Candidate), B.H. Rowe, MD, MSc, CCFP(EM); University of Alberta

Keywords: allergy/anaphylaxis, emergency medicine, EMS, surveillance

Introduction: Anaphylaxis is a serious, albeit uncommon, trigger for emergency medical services (EMS) activation, and case ascertainment is important for surveillance and quality assurance. This study explored the coding and management of patients with allergy and anaphylaxis in an EMS service. Methods: From 377,000 Alberta Health Services - Edmonton Zone EMS calls in 2011, patient care records (PCRs) were retrospectively reviewed to identify calls meeting anaphylaxis and allergy criteria. Various diagnostic codes (allergy; breathing problem; choking; sick person; unconscious; unknown problem) were used to identify all potential cases. Data were collected by trained staff from standardized, electronic PCRs using the REDCap data platform. Anaphylaxis was confirmed using current guideline criteria. Descriptive data are reported using proportions and medians with interquartile range (IQR), where appropriate, and compared using chi-square and Mann-Whitney tests. Results: Overall, 481 cases were reviewed; 136 (28\%) met criteria for anaphylaxis, 109 (23\%) were allergy/nonanaphylaxis, 143 (30\%) were nonallergic, and 93 (19\%) provided insufficient evidence for diagnostic confirmation. Cases from both groups were distributed throughout various EMS codes. Anaphylaxis and allergy occurred similarly in women (65 v. 63\%); anaphylaxis patients were younger ( 30 v. 38 years; $p<0.05$ ). More anaphylaxis patients had a previous history of "allergy" (76 v. $57 \% ; p<0.001$ ). Prior to EMS arrival, more anaphylaxis patients had self-administered treatment (44 v. $26 \% ; p=0.003$ ), especially epinephrine ([EPI], 25 v. 2\%; $p<0.001$ ). All EMS treatments were administered more frequently to anaphylaxis patients, including $\mathrm{H}_{1}$ antihistamines (74 v. $51 \%$ ), intravenous saline (45 v. $15 \%$ ), EPI (36 v. 1\%), inhaled beta-agonists (32 v. 3\%), and corticosteroids (18 v. $3 \%)$; all $p<0.001$. Overall, more anaphylaxis patients received EPI (54 v. 3\%), corticosteroids (20 v. 4\%), and antihistamines $(88$ v. $59 \%)$, and all three $(15 \mathrm{v} .1 \%)$ received prior to their ED arrival (either self-administered or by EMS); all $p<$ 0.001 . More anaphylaxis cases $(94$ v. $73 \% ; p<0.001)$ were transported. Conclusion: While patients with anaphylaxis are clinically and therapeutically distinct from those with allergy, they are difficult to identify using current EMS coding. Efforts to improve EMS coding of anaphylaxis would enhance surveillance and quality assurance of this serious condition.
P35 A comparison of high-sensitivity cardiac troponin I assay with the current sensitive cardiac troponin $I$ test in the emergency department

A. Worster, MD, W. Krizmanich, MD, I.J. Preyra, MBA, MD, P. Kavsak, PhD; McMaster University

Keywords: acute coronary syndrome, emergency department, emergency medicine, troponin

Introduction: Presently, Health Canada has approved only Roche Diagnostics' high-sensitivity cardiac troponin T (hs-cTnT) assay, whereas in Europe, both hs-cTnT and Abbott Diagnostics' hs-cTnI assay (in 2013) have been approved. Being more sensitive than current cTn assays, hs-cTn assays are able to detect $\mathrm{cTn}$ in more patients. Studies have demonstrated significant differences in cutoffs between the cTnT and hs-cTnT assays with important diagnostic implications. According to Roche Diagnostics' package insert, the prevalence of myocardial injury has nearly doubled in patients presenting with chest pain to the ED (approximately $20 \%$ using the cTnT assay to over $40 \%$ using the hs-cTnT assay). The TOES study (Troponin Observational study in the Emergency Setting) was conducted to determine if there are also significant differences in overall positivity between hs-cTnI versus cTnI in the ED. Methods: After receiving research ethics approval, we measured hs-cTnI (ARCHITECT STAT high sensitive Troponin-I; research use only) in parallel and concurrent with cTnI (ARCHITECT STAT Troponin-I) assay for clinical care within the adult EDs of a single Ontario city for a 3-month period. Clinicians and researchers remained blind to the hs-cTnI results. This preliminary analysis represents measurements from the first 40 days after live-date. Results: There were 5,280 cTn measurements from 3,203 ED patients (median [interquartile] age $=70[56-81]$; females $=51 \%$ ) during this time frame. Passing and Bablok analysis on 5,276 paired results yielded the following equation: hs-cTnI $=1.13(\mathrm{cTnI})-1.67 \mathrm{ng} / \mathrm{L}$. The hs-cTnI assay was measurable in 94\% (95\% CI: 93-94) of samples with $30 \%$ (95\% CI: 29-32) of concentrations $>99$ th percentile $(\geq$ $26 \mathrm{ng} / \mathrm{L})$ compared to only $48 \%$ (95\% CI: 47-49) measurable with $25 \%(95 \%$ CI: $24-27)>99$ th percentile $(>0.03 \mu \mathrm{g} / \mathrm{L})$ using the cTnI assay. Conclusion: The percentage of measurable and abnormal cTn concentrations will increase when using a hs-cTnI assay in the ED. However, the prevalence of myocardial injury appears to be significantly less than what has been reported for the hs-cTnT assay.

P36 The role of procalcitonin and the MASCC score in emergency department patients with chemotherapy-induced febrile neutropenia

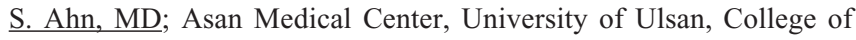
Medicine, Seoul, Korea

Keywords: bacteremia, emergency medicine, febrile neutropenia, procalcitonin

Introduction: Infectious complication could be life-threatening in patients with chemotherapy-induced febrile neutropenia (FN). The Multinational Association of Supportive Care in Cancer (MASCC) risk-index score is used to predict complications in these patients, indicating a MASCC score of $\geq 21$, a low-risk episode for serious complications. However, bacteremia was not included as one of the serious outcomes in the derivation study. In this study, we evaluated procalcitonin and the MASCC score in predicting bacteremia and septic shock in patients with FN. Methods: From November 2010 to October 2011, 400 episodes of FN are prospectively enrolled. Clinical and laboratory findings including procalcitonin and MASCC score were analyzed and correlated with the infectious complications of FN. Results: Of the 400 episodes, 36 (9.0\%) had bacteremia and $25(6.3 \%)$ developed septic shock. Serum PCT $\geq 0.5 \mathrm{ng} / \mathrm{mL}$ (OR 4.07, 95\% CI 1.57-10.53), $\mathrm{CRP} \geq 10 \mathrm{mg} / \mathrm{dL}$ (OR 2.74, 95\% CI 1.05-7.18), MASCC score $<21$ 
(OR 2.55, 95\% CI 1.10-5.91), and platelet count $<100 \times 103 / \mathrm{mm}^{3}$ (OR 2.53, 95\% CI 1.13-5.66) were independently predictive of bacteremia, and serum PCT $\geq 1.5 \mathrm{ng} / \mathrm{mL}$ (OR 29.49, 95\% CI 7.96-109.31) and MASCC score $<21$ (OR 13.40, 95\% CI 3.93-45.70) were independent factors of septic shock in multivariate analysis. Conclusion: As bacteremia and septic shock are the most life-threatening complications in FN, implicating PCT as a routine use in clinical practice along with the MASCC score could improve the risk stratification of patients with chemotherapy-induced FN

\section{P37 Adverse events in the emergency department: a systematic review}

A.S. Stang, MD, MBA, MSc, A.S. Wingert, BA, MPH, L. Hartling, $\mathrm{PhD}$, A. Plint, MD; University of Calgary

Keywords: adverse events, emergency medicine, patient safety Introduction: Adverse events (AE), defined as unintended harms resulting from the care and services provided to the patient, represent a significant threat to patient safety and public health. The emergency department (ED) is considered high risk for patient safety events. Methods: We systematically searched major bibliographic databases, relevant journals and conference proceedings, and completed reference reviews of primary articles. Randomized controlled trials, cohort studies, and case-control studies were included if they examined a broad demographic group reflecting a significant proportion of ED patients and described the proportion of AE. Studies conducted outside of the $\mathrm{ED}$, those that examined only a subpopulation of ED patients (e.g., a specific entrance complaint or receiving a specific intervention), or examined only adverse drug events, were excluded. Two independent reviewers assessed study eligibility, completed data extraction, and assessed study quality with the Newcastle Ottawa Scale. Heterogeneity across the studies in design, patient follow-up, and method of outcome assessment precluded meta-analysis. Results: Our search identified 11,624 citations. Ten articles, representing eight unique cohort studies, were identified for inclusion and qualitative review. Methodological quality was low to moderate, with weaknesses in study group comparability, follow-up, and outcome ascertainment and reporting. The overall proportion of ED patients with AE ranged from 0.16 to $8.5 \%$. Studies utilizing passive reporting systems demonstrated a lower proportion of patients with $\mathrm{AE}$ than those utilizing more active methods such as chart review or structured interviews. The reported preventability of $\mathrm{AE}$ ranged from 36 to $71 \%$. The severity rating scales used were variable and poorly reported. Among the seven studies reporting types of $\mathrm{AE}$, the most common types of events were related to management (three studies), diagnosis (two studies), and medication (two studies). While four studies enrolled both pediatric and adult patients, there were no pediatric-specific studies, and age-specific data were insufficient for subgroup analysis. Conclusion: There is substantial variation in the reported prevalence, preventability, and severity of $\mathrm{AE}$ in the ED. Further studies with rigorous, standardized outcome assessment and reporting are required, particularly for potentially vulnerable populations such as children and the elderly.

P38 Parenteral dextrose utilization in a large, urban EMS system E. Williams, MD, A.O. Arthur, PharmD, S.H. Thomas, MD, MPH, J.M. Goodloe, MD; The University of Oklahoma School of Community Medicine

Keywords: dextrose, emergency medicine, EMS, hypoglycemia Introduction: Parenteral dextrose administration by advanced emergency medical technicians (AEMTs) and paramedics is routine in emergency medicine services (EMS). Anecdotal, although widespread, belief among AEMTs and paramedics in our large, urban EMS system is that a sizeable cohort of diabetic patients summon EMS frequently, as often as weekly, for clinical conditions requiring parenteral dextrose. This study's purpose was to comprehensively review utilization patterns of parenteral dextrose in a large, urban EMS system to include the following: overall administration frequency, unique patient administration frequency, dosage(s) in administration, and influence of patient characteristics (demographic variables) upon administration need. Methods: Retrospective chart review of patient encounters occurring January 5, 2010, to July 5, 2011, in a large, urban EMS system. The patient cohort was defined by AEMT and/or paramedic parenteral administration of dextrose. Results: In the 18-month study period, 3,203/230,048 (1.4\%) patient encounters involved parenteral dextrose administration. A total of $669(20.9 \%)$ cohort patients received parenteral dextrose on more than one EMS encounter, with 6 $(0.2 \%)$ encountered more than 20 times during the study period, and $2,876(89.8 \%)$ cohort patients received $25 \mathrm{~g}$ or less of parenteral dextrose to resolve hypoglycemia (system definition $=$ blood glucose less than $50 \mathrm{mg} / \mathrm{dL}) ; 2,352(73.4 \%)$ were greater than 60 years of age; $2,354(73.5 \%)$ were Caucasian; and 2,120 (66.2\%) were transported to a hospital-based emergency department, with the remaining 1,083 (33.8\%) refusing offer of EMS transportation. Conclusion: A small percentage of patient encounters in this large, urban EMS system involve AEMTs and/or paramedics administering parenteral dextrose. While 1 in 5 patients in the study cohort did require multiple EMS encounters for hypoglycemia-related conditions, the belief that a sizeable percentage are utilizing EMS as frequently as weekly for the same is false. Nine in 10 encounters requiring parenteral dextrose administration can be clinically resolved with the use of $25 \mathrm{~g}$ or less. Demographic analysis reveals increased needs for EMS hypoglycemia care in Caucasians greater than 60 years of age.

P39 EMS stretcher "misadventures" in a large, urban EMS system: a longitudinal analysis of contributing factors and resultant injuries

T. Kallsen, BS, A.O. Arthur, PharmD, S.H. Thomas, MD, MPH, J.M. Goodloe, MD; The University of Oklahoma School of Community Medicine

Keywords: emergency medicine, EMS, injury prevention, stretcher operations

Introduction: There is a paucity of data regarding injury affiliated with stretcher operations to patients and EMS professionals. This longitudinal study's purpose is to continue to describe and analyze characteristics associated with undesirable stretcher operations, with or without resultant injury to patients and/or EMS professionals in a large, urban EMS agency. Methods: In the EMS agency studied, all stretcher-related "misadventures" are required to be documented by EMS personnel, regardless of whether injury results, using free text incident reporting software. All such stretcher-related reports for incidents that occurred July 1, 2009, to June 30, 2012, were queried from the agency's risk management database for retrospective analysis, avoiding the Hawthorne effect in stretcher operations. Results: During the 3 years studied, the EMS agency transported 404,178 patients. Fifty-nine stretcher incidents were reported ( 0.15 per 1,000 transports). No substantive patient injury occurred. Eight EMS providers sustained minor injuries, including five back injuries, two knee injuries, and two arm contusions. Only one personnel requested time off work, for a 4day duration. There were three primary times of stretcher operation problems: unloading, loading, and surface movement. Forty-one of 59 $(69.5 \%)$ occurred during unloading. Five of 59 (8.5\%) occurred during loading. Thirteen of $59(22.0 \%)$ occurred during surface movement. There were five predominant contributing aspects to stretcher operation problems, with some incidents stemming from multiple aspects: stretcher-ambulance safety latch mechanism, ground surface conditions, equipment failure, bariatric patient size, and combative patient 
behaviour. Nineteen of 59 (32.2\%) related to the stretcher not engaging locking mechanisms on the ambulance floor. Thirteen of $59(22.0 \%)$ related to poor ground surface conditions. Five of $59(8.5 \%)$ related to equipment malfunction. Three of $59(5.1 \%)$ related to patient weight exceeding $450 \mathrm{lb}$, compounded by patient movement on the stretcher. Two of 59 (3.4\%) related to combative patient movement. Conclusion: In a large, urban EMS system, the incidence of injury related to stretcher operations is markedly low, with few personnel injuries and no substantive patient injuries incurred during the 3-year study period. EMS personnel should be particularly aware of increased risk for stretcher misadventure during patient unloading.

P40 Reduced times for acute stroke patients who receive thrombolysis at Royal Melbourne Hospital by the implementation of a direct ambulance to $\mathrm{CT}$ process

M. Truesdale, MBBS, FACEM, B. Campbell, MBBS, FRACP, A. Meretoja, MD, S. Davis, MD, FRACP; University of Melbourne, Department of Medicine

Keywords: acute stroke, emergency management, emergency medicine, thrombolysis

Introduction: The Emergency Department (ED) and Stroke Care Unit (SCU) at Royal Melbourne Hospital (RMH) have worked collaboratively over recent years to introduce processes that streamlined the management of stroke patients, including the implementation of thrombolysis (tPA) for those meeting strict criteria. In spite of this collaboration and knowledge that tPA is more effective when delivered early after symptom onset, in-hospital delays of $>60$ minutes before administration were common. The Helsinki model of thrombolysis with a median 20 minutes door-to-needle time (DNT) was initially described at the 2012 European Stroke Conference and formed the basis for change. Methods: The existing "code stroke" model at RMH was evaluated and restructured in early 2012 to include (1) ambulance prenotification with patient details alerting the stroke team to meet the patient on arrival at $\mathrm{RMH}$; (2) patients transferred directly from triage onto the CT table on the ambulance stretcher; and (3) tPA delivered on the CT table immediately after imaging. The prospective consecutive tPA registry for effects of these protocol changes on the DNT following implementation during business hours ( 8 am-5 pm Mon-Fri) from May 2012 were reviewed. Results: There were 48 patients treated with tPA in the 8 months following the protocol change. Compared to 85 patients treated in 2011 , the median (IQR) DNT was reduced from 61 (43-75) minutes to 46 (24-79) minutes $(p=0.040)$. All of the effects came from the change in the in-hours DNT, down from 43 (33-59) to 25 (19-48) minutes $(p=$ $0.009)$, while the out-of-hours delays remain unchanged, 67 (55-82) to 62 (44-95) minutes $(p=0.835)$. Conclusion: An optimized tPA protocol with the key components of prenotification and direct-to-CT transfer was implemented at RMH and resulted in a decrease in the time to thrombolysis for patients with acute stroke in hours. Until January 1, 2013 , workforce issues had limited the provision of the same service level 24/7; however, it has now been extended.

P41 ACEing it: can abdominal and cardiothoracic assessment with sonography in shock be learned using high-fidelity simulation?

P.R. Atkinson, MD FCEM, G. Verheul, MD, J. Fraser, Bachelor of Nursing, D. Lewis, MBBS FRCS FCEM; Discipline of Emergency Medicine, Memorial University

Keywords: emergency medicine, point-of-care ultrasound, simulation Introduction: Shock ultrasound training can be limited by the availability of patients with pathology. Simulation may provide a more efficient way of training. We assessed ultrasound simulation for teaching ultrasound technique when learning the Abdominal and Cardiothoracic Evaluation by Sonography in Shock (ACES) protocol. Methods:
Forty-two physicians participated in educational sessions that included didactic learning, followed by supervised scanning on normal volunteers and patients with pathology using portable ultrasound machines (GE and SonoSite). Participants performed focused ACES scans using a high-fidelity ultrasound simulator (VIMEDIX), imaging the heart, chest, abdomen, aorta, and inferior vena cava (IVC). All participants were then surveyed using a standardized 5-point Likert scale on using simulation and volunteer/patients for learning technique and for training overall. Data were analyzed using paired $t$-test (GraphPad Prism). Results: All 42 surveys were completed. Twenty-seven (64.3\%) had previous ultrasound training, and two (4.8\%) had previously used the ultrasound simulator. As a method for learning ultrasound technique, there was no significant difference in mean scores between patient/volunteers and the simulator for cardiac ( 4.5 v. $4.4 ; p=0.435)$, chest (4.4 v. $4.3, p=0.249)$, aorta ( 4.5 v. $4.3 ; p=0.154)$, or IVC ( 4.2 v. $4.4 ; p=$ 0.465 ) scanning. For abdominal (free fluid) scanning, learning scanning technique was superior with patients/volunteers (4.6 v. $4.3, p=$ 0.032 ). Forty-one (97.6\%) participants stated that they would use highfidelity ultrasound simulation to further develop and maintain skills. Conclusion: Simulated ultrasound compared favourably to real ultrasound for learning scanning technique for all but one of the ACES windows. It is likely that ultrasound simulation will have an increasingly important role in shock ultrasound education and skills maintenance, although it is unlikely to replace the need for training on live patients.

P42 Presenting characteristics of patients undergoing cardiac troponin measurements in the emergency department

D. Goodman, BHSc, A. Worster, MD, S. Hill, PhD, P. Kavsak, PhD; McMaster University

Keywords: demographics, emergency department, emergency medicine, troponin

Introduction: Cardiac troponin (cTn) is a biomarker of myocardial injury. Around the world, millions of Tn assays are conducted annually on patients in emergency departments (EDs) with ACS symptoms to rule in or rule out myocardial infarction. For patients without ACS symptoms, the value of elevated cTn levels in treatment decisions is unclear, yet cTn levels are often measured on these patients, especially the elderly (age $>70$ years). Methods: To better understand the characteristics of patients in the ED who undergo cTn testing, we reviewed 15,275 cTn measurements conducted in all EDs of a single Canadian city during 2011 and recorded patient age, sex, presenting CEDIS complaint, and final disposition. Results: The average age of patients undergoing cTn testing was 63 years; $49 \%$ were female. The most common CEDIS complaints were chest pain (cardiac and noncardiac) $(n=3,711)$, shortness of breath $(n=1,740)$, abdominal pain $(n=$ $1,383)$, and generalized weakness $(n=936)$. A secondary analysis was also done to determine if there was a difference in troponin testing in the elderly with non-ACS-related complaints. The percentage of patients who underwent cTn testing for non-ACS complaints differed significantly based on age $(\chi 2=82.7, p=<0.0001 ; \mathrm{OR}=1.2(95 \%$ CI: $1.16-1.25$ for patients $>70$ years). Conclusion: Elderly patients without ACS symptoms are more likely to undergo cTn testing in the ED than their younger counterparts, but the contribution of the cTn measurement to treatment decisions is unclear.

\section{P43 Reporting and learning from prehospital patient safety} incidents

G.S. Hunte, MD, PhD, J.R. Brubacher, MD, L. Hamilton, BSc, MSc, D. Andrusiek, MSc, G. Miller, RN, BA, MSN, A. Taylor, RN, BScN, MA, CHE; University of British Columbia

Keywords: emergency medicine, patient safety, prehospital 
Introduction: British Columbia has implemented a provincial Webbased safety learning system across health care sectors, including prehospital care. We sought to inform safety learning in prehospital care by describing how prehospital care providers make sense and give account of patient safety incidents (PSIs) in everyday practice. Methods: We conducted 20 in-depth semistructured one-on-one interviews and 3 group interviews with 27 prehospital care providers, supervisors, and dispatchers using the EMS Safety Attitudes Questionnaire as a stimulus to explore perceptions and experiences of safety in prehospital care. Results: Quantitative findings demonstrate low to moderate point estimates on both domain mean scores and the proportion of positive response. In the emergent conversation about prehospital safety from interviews and focus groups, we found that reporting and learning from prehospital PSIs are impeded by a fragmented, hierarchical, and often punitive and remedial structure and process of oversight and organizational response. Disciplinary response, in particular, constrains system learning, and fear and futility of reporting are endorsed by most participants. Whereas medical oversight is generally valued for emphasis on clinical reasoning, organizational response confounds individual and system learning through multiple, opaque, and conflicting channels, with lack of coordination and consistency between reviews. Historical work conflicts foster an adversarial dynamic between providers and management. Hence, learning from safety incidents remains primarily parallel and informal rather than collaborative and systematic. In short, lack of feedback, punitive response, and distrust are the primary barriers to organizational learning from safety incidents. Conclusion: Reporting and learning from prehospital PSIs will benefit from a shift away from a punitive organizational response toward a collaborative model of learning such as a peer-based or third-party review process. A transparent structure and process will offer practitioners a venue to contribute to improving patient care and creating safety in prehospital emergency care.

P44 Development of a new indicator measuring in-hospital mortality of patients with emergency-sensitive conditions

$\underline{\text { S. Berthelot, MD }}$, H.T. Stelfox, MD, PhD, E. Lang, MD, H. Quan, $\mathrm{MD}, \mathrm{PhD}$; Université Laval

Keywords: emergency medicine, emergency service, health care, hospital, hospital mortality, quality indicators

Introduction: The Canadian Institute for Health Information (CIHI) provides estimates of four types of hospital-standardized mortality ratio (HSMR) to each Canadian hospital. However, none of these specifically captures the outcomes of admitted patients whose emergency department (ED) management would be expected to have an impact (emergency-sensitive conditions). We propose the development of a HSMR specific to emergency-sensitive conditions. Methods: Adapted from CIHI's methodology, the ED-HSMR for 2010-2011 is the ratio of the actual number of deaths among patients with emergencysensitive conditions in one hospital during the year 2010-2011 to the expected number of deaths for the same conditions in the same hospital during the reference year 2009-2010. The ratio is then multiplied by 100. The expected deaths are estimated from mortality probabilities during the reference year for patients with emergency-sensitive conditions in the hospital-peer group and adjusted through logistic regression for patient variables known to impact in-hospital mortality. An ED-HSMR will be calculated for all Canadian hospitals, with at least 20 expected deaths estimated from the predictive model. Sensitivity analyses will be performed to evaluate the impact of individual emergency-sensitive conditions on ED-HSMR estimates. Results: A list of 37 emergency-sensitive conditions has been selected for the calculation of the ED-HSMR. We have developed a predictive model to estimate the expected number of deaths, adjusted for the following covariates: age, gender, length of in-hospital stay, comorbidity group (Charlson index), and transfers from another institution. Ten provinces and 51 health regions throughout Canada are included. Four hospital-peer groups are assessed: one group for teaching hospitals and three groups for community hospitals classified on admission volume and patient complexity. ED-HSMR estimates and analyses will be subsequently presented. Conclusion: The calculation of an in-hospital standardized mortality ratio specific to emergency care may help guide assessment and improvement of ED performance.

\section{P45 Prehospital management of anaphylaxis in one Canadian urban centre}

T. Chung, BSc (Candidate), L. Lovstrom, BSc, MSc (Candidate), C. Vandenberghe, MSc, S. Couperthwaite, BSc, S. Sookram, MD, FRCPC, K. Liss, BSc, MBA, C. Villa-Roel, MD, PhD (Candidate), B.H. Rowe, MD, MSc, CCFP(EM); University of Alberta

Keywords: allegy, anaphylaxis, emergency medicine, EMS

Introduction: Anaphylaxis presents as a sudden allergic reaction involving more than two body systems and can be fatal if not managed appropriately. Prompt medical attention and administration of intramuscular epinephrine (EPI) are critical for improved outcomes. Limited research has been conducted on the emergency medical services (EMS) management of anaphylaxis, especially in Canada. Methods: From 377,000 Alberta Health Services - Edmonton Zone EMS calls in 2011, patient care records (PCRs) were retrospectively reviewed to identify calls meeting anaphylaxis criteria. Data were collected by trained staff unaware of the study hypothesis from standardized, electronic PCRs using the REDCap data platform. Anaphylaxis was confirmed using current guideline criteria. Duplicate data extraction was completed on the first 20 PCRs to enhance data abstraction reliability. Descriptive data are reported using proportions and medians with interquartile range (IQR). Results: Of the 481 cases reviewed, 136 $(28 \%)$ met criteria for anaphylaxis confirmation. Anaphylaxis occurred more often in women (89 [65\%]); median age was 39 years (IQR: 16, 50). Advanced life support EMS response (135 [99\%]) and high severity predominated. Most patients had a previous history of "allergy" (105 [77\%]), and all identified their perceived trigger for the current attack. Prior to EMS arrival, anaphylaxis patients had self-administered treatment, including EPI (34 [57\%]; 4 [12\%] had received two treatments), $\mathrm{H}_{1}$ antihistamines (39 [65\%]), inhaled beta-agonists (7 [12\%]), and corticosteroids (3 [5\%]). The EMS treatment included $\mathrm{H}_{1}$ antihistamines $(98[74 \%])$, intravenous saline (61 [45\%]), EPI (49 [36\%]), inhaled beta-agonists (43 [32\%]), and corticosteroids (24 [18\%]). Repeat epinephrine was required infrequently (12 [19\%]). Overall, 73 (54\%) patients received EPI, 27 (19\%) received corticosteroids, 120 $(88 \%)$ received antihistamines, and $10(15 \%)$ received all three prior to their ED arrival (either self-administered or by EMS). Most cases (128 [94.1\%]) were transported. Conclusion: Patients with anaphylaxis are relatively infrequent in this EMS service over a 1-year period. Overall, despite an EMS protocol for anaphylaxis management, EPI use was suboptimal and often delayed. Efforts to enhance the use of EPI in the prehospital setting seem warranted.

\section{P46 "Safety culture" assessment: questionnaires or "quester-} views"?

G.S. Hunte, MD, PhD, J.J. Joyce, PhD, W.H. McKellin, PhD, S.B. Sheps, MD, MSc; St. Paul's Hospital

Keywords: emergency medicine, patient safety, research methodology Introduction: Most "safety culture" research uses survey instruments to collect individual perceptions, attitudes, and beliefs. Few tools specify a theoretical model, and none of the currently available instruments in health care has robust psychometric properties. "Questerviews" use a standardized survey instrument as a stimulus 
to which participants are free to explore their responses. Methods: We compare measurement of safety culture using a standardized questionnaire with interpretive findings using a questerview strategy. A purposive sample of 26 urban, tertiary care ED care providers and administrators participated in questerviews using the Hospital Survey on Patient Safety Culture (HSOPSC). Results: The HSOPSC was a useful semiotic tool to elicit multiple interpretive frames. Participants had a lot to say that did not fit into the survey questions. Reflecting on stories and safety relevant issues that were not captured by the survey instruments, such as decision making and security, offered additional insight into how providers create safety in everyday practice. Several questioned from which perspective or role they were to answer. The lack of contextual background made some survey statements difficult to interpret, and competing examples led to neutral responses. Participants often expressed a more negative view of the organization's safety culture upon reflection. Participants also noted that although they may have had difficulty choosing a Likert response, they were able to bring a rich, nuanced story to mind. Conclusion: There is a tension between the holistic character of culture and the standard reductionistic approach of its measurement. The act of measurement implies some degree of observability and comparison to some agreed upon reference scale, which raises the question if safety culture can be measured at all Use of standard measures within in-depth interviews provoked narratives that were both more complex and illuminating, recommending questerviews as a pragmatic way to integrate qualitative and quantitative methods.

P47 Government benefit payments and acute injury among lowincome mothers presenting to the emergency department

W.K. Chan, BHSc, E. Shafir, PhD, S. Mullainathan, PhD, D.A. Redelmeier, MSc, MD; Sunnybrook Health Sciences Centre

Keywords: emergency medicine, injury, social benefits

Introduction: Human error due to risky behaviour is a common and important contributor to acute injury related to poverty. We studied whether government benefit payments mitigate or exacerbate risky behaviours that lead to emergency visits for acute injury among lowincome mothers with dependent children. Methods: We analyzed total emergency department visits throughout Ontario to identify women between 15 and 55 years of age who were mothers of children younger than 18 years, who were living in the lowest socioeconomic quintile, and who presented with acute injury. We used universal health care databases to evaluate emergency department visits during specific days on which government benefit payments were made (defined as a child benefit distribution) relative to visits on control days over a 7-year interval (1 April 2003 to 31 March 2010). Results: A total of 153,377 emergency department visits met the inclusion criteria. We observed fewer emergencies per day on child benefit payment days than on control days (56.4 v. $60.1, p=0.008$ ). The difference was primarily explained by lower values among mothers age 35 years or younger (relative reduction $7.29 \%, 95 \%$ confidence interval [CI] 1.69 to 12.88), those living in urban areas (relative reduction $7.07 \%, 95 \%$ CI 3.05 to 11.10 ), and those treated at community hospitals (relative reduction $6.83 \%$, 95\% CI 2.46 to 11.19). No significant differences were observed for the 7 days immediately before or the 7 days immediately after the child benefit payment. Conclusion: Contrary to political commentary, we found that small reductions in relative poverty mitigated, rather than exacerbated, risky behaviours that contribute to acute injury among lowincome mothers with dependent children. Implications on emergency department dynamics, such as staffing, remain a topic for further research.
P48 Quantitative assessment of lumbar puncture skill in experts and nonexperts using needle trajectories: implications for competency-based medical education

D. Clinkard, MSc, E. Moult, BEng, T. Ungi, PhD, G. Fichtinger, PhD, R. McGraw, MD; Queen's University

Keywords: competency-based education, emergency medicine, lumbar puncture, quantitative

Introduction: For procedural skills, a CBME program requires an understanding of the level of skill required for a specific level of training, an understanding of how many times a student will have to practice the skill to achieve that level competence, and, finally, a way of measuring achievement. We sought to determine if repetitive practice altered lumbar puncture (LP) skill retention in novices as assessed by dichotomous checklist and if quantitative electromagnetic needle tracking could be used as a measure of performance. Methods: Thirty-two medical students were divided into two groups. One group was asked to practice LP once and the other five times. Eight attending ER physicians and eight residents were used as comparative groups. LP performance was assessed with $3 \mathrm{D}$ electromagnetic tracking, which allowed for a graphical representation of needle path length. A validated, dichotomous checklist was also used for assessment. Students were recalled at 1 month to assess skill retention. Results: Students who practiced multiple times had significantly fewer checklist mistakes initially and at 1 month. Quantitative needle metrics as assessed by electromagnetic tracking showed a decreasing trend in needle movement distance with practice and with experience. Conclusion: These findings suggest that repetitive practice improves skill retention and that quantitative needle metric tracking may have a potential role in evaluating LP skill.

P49 Knowing is half the battle: utilizing patient outcome information in emergency medicine

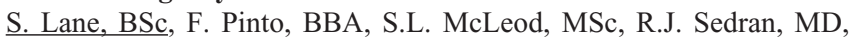
MSc, BESc, FRCPC; The University of Western Ontario

Keywords: emergency medicine, outcome information

Introduction: The nature of emergency medicine (EM) is that of onetime clinical encounters where patient outcomes are often unknown. Seeking patient outcome information (POI) occurs when physicians determine what happens to a patient after the clinical encounter ends. The objective of this study was to determine the attitudes and behaviours of emergency physicians and residents rotating through the emergency department in regards to obtaining POI. Methods: A 22-item online survey was sent to emergency physicians and residents working at an academic tertiary care centre (annual census 125,000) to determine the perceived utility of POI and what methods and practices physicians and residents currently employ to obtain POI. Results: Eighty-five participants completed the survey, of whom 41 were staff physicians and 44 were residents. The majority of these residents (53\%) were in an EM training program. The perceived utility of POI included improving diagnostic accuracy (94\%), improving the management of future patients with similar presentations (95\%), and assessing the appropriateness of referrals to other services (86\%). Interestingly, $16.5 \%$ of respondents claimed that POI would not be beneficial in improving clinical practice. Respondents most commonly sought out POI if a shift ended with outstanding patient laboratory or diagnostic imaging results, with the electronic medical record (EMR) being the preferred tool to obtain POI. Fifty-seven percent of resident respondents stated that they would most likely seek POI for patients sent home with instructions to return for further diagnostic testing (e.g., ultrasound) compared to $27 \%$ of staff respondents ( $\Delta 30 \% ; 95 \%$ CI: 9 , 47). Respondents favoured better utilization of the EMR to improve 
POI (e.g., being automatically notified of discrepant imaging results [89\%]). Barriers to seeking POI included time constraints (77\%), difficulties accessing the patient information $(66 \%)$, and weaknesses in the EMR (62\%). Conclusion: POI was identified as useful for improving clinical acumen and management skills. Major barriers to seeking POI were identified as time and EMR functionality. Optimization of POI may improve both patient care and physician learning.

\section{P50 Treatment of acute COPD exacerbations in rural Alberta emergency departments}

P. McKenna, MD (Candidate), K. MacLeod, MD (Candidate), C. Le, MD (Candidate), K. Tok, MD (Candidate), J. Ursenbach, MD (Candidate), L. Sutherland, MD (Candidate), L. Lovstrom, BSc, MSc (Candidate), S. Couperthwaite, BSc, C. Villa-Roel, MD, PhD (Candidate), B.H. Rowe, MD, MSc, CCFP(EM); University of Alberta

Keywords: COPD, emergency medicine, guidelines, rural ED Introduction: Acute exacerbations of COPD (AECOPD) are common presentations to emergency departments (ED); however, limited information exists for the management in nonurban locations. We sought to examine the diagnostic and treatment approaches to patients with AECOPD in three rural EDs and determine levels of adherence to recommendations contained within clinical practice guidelines. Methods: Using retrospective chart review methods, we evaluated the difference in treatment of AECOPD among three EDs in rural Alberta. Cases were chosen for review based on ICD-10 codes J41-44. Data are reported as median and interquartile ranges (IQR) and proportions. Results: Over a 1-year study period, 192 patients presented a total of 266 times with AECOPD. The average age was 68 (IQR: 58, 77), and $54.9 \%$ were females. Diagnostic testing included chest $\mathrm{x}$-ray in $65.0 \%$, bloodwork in $45.1 \%$, ECG in $33.5 \%$, and ABGs in $6.4 \%$; few received pulmonary function testing. In the $\mathrm{ED}, 58.7 \%$ of patients were treated with a short-acting beta-agonist (SABA), $48.9 \%$ with a short-acting anticholinergic (SAAC), $27.4 \%$ with corticosteroids, and $19.9 \%$ with antibiotics. Overall, SABAs (63.5\%), anticholinergic agents $(53.4 \%)$, corticosteroids $(54.5 \%)$, and antibiotics $(71.1 \%)$ were prescribed more commonly to discharged patients (all $p<0.05$ ). Conclusion: There are low to moderate levels of adherence to clinical practice guidelines in the rural ED management of AECOPD. Further research might compare these results to rural and urban settings or examine whether readily available point-of-care treatment maps or checklists improve adherence to CPG recommendations.

P51 Initial assessment and disposition for the stable patient with first-trimester bleeding in the emergency department

R.M. Gagnier, MD, CCFP(EM), S.L. McLeod, MSc, C.A. Richardson, $\mathrm{MD}$, CCFP(EM); The University of Western Ontario

Keywords: emergency medicine, first-trimester bleeding, miscarriage Introduction: First-trimester bleeding in a stable patient is a common complaint in the emergency department (ED). However, there are no clinical guidelines for initial assessment and disposition of this population. The objectives of this study were to determine variations in the initial assessment and disposition of first-trimester bleeding by emergency physicians. Methods: A retrospective medical record review was conducted from a random sample of stable patients aged 18 to 45 years presenting to the ED with first-trimester bleeding. Patient history (quantity of bleeding, presence of tissue or clots, abdominal pain), physical examination findings (abdominal examination, speculum examination, bimanual, ultrasound), investigations (CBC, Rh, BhCG, formal US), and disposition (early pregnancy assessment unit [EPAU], family physician, inpatient $\mathrm{OB} / \mathrm{GYN}$ consult, or follow-up in ED in 24 hours with formal US) were recorded by trained research personnel. Emergency physicians were invited to complete a 26-item question- naire regarding their initial assessment and disposition for this population. Results: A total of 135 charts were reviewed; 92 were included. Ninety-one percent of patients had spotting, 23\% clots, and 58\% abdominal pain. On physical examination, $99 \%$ had an abdominal examination, 55\% transabdominal (TA) point-of-care ultrasound (PoCUS), 35\% a bimanual, 26\% speculum, and 7\% transvaginal (TV) PoCUS. Investigations included 92\% serum 3 hCG, $88 \%$ CBC, $86 \%$ $\mathrm{Rh}$, and $10 \%$ formal ultrasound (US). For disposition, $75 \%$ were sent to EPAU, $14 \%$ to family physician, $8 \%$ returned to the ED for formal US, and 3\% inpatient obstetrician assessment. Seventy-six percent of physicians completed the survey; $73 \%$ were PoCUS certified. Responses regarding clinical examination, investigations, and disposition were similar to chart review. However, respondents would perform PoCUS more frequently than found in chart review (96\% v. 55\% TA, $27 \%$ v. $7 \%$ TV). Eighty-three percent of respondents were comfortable performing a TA US but only $31 \%$ a TV US. Most stated that they would refer $\geq 75 \%$ to EPAU. Respondents were comfortable waiting 2 to 4 days for an EPAU visit for patients with spotting (86\%), confirmed IUP on PoCUS $(80 \%)$, or moderate bleeding $(69 \%)$. Conclusion: These results reflect variations in the ED management for stable patients with first-trimester bleeding, suggesting room for improvement and the need for standard management guidelines.

\section{P52 Caring for disadvantaged populations in the emergency department \\ J. Krause, MD, S.L. McLeod, MSc, R.J. Sedran, MD, MSc, BESc, FRCPC; The University of Western Ontario}

Keywords: attitudes, disadvantaged population, emergency department, emergency medicine

Introduction: Caring for disadvantaged populations in the emergency department often provides significant medical and interpersonal challenges, and the physician attitude toward the patient can be strained. Disadvantaged populations can include IV drug abuse, extreme poverty, and homelessness. The objective of this study was to determine physician attitudes when caring for disadvantaged populations and to determine if understanding the social background of the patient increases the physician's empathy toward the patient. Methods: A survey was designed using several case presentations of patients from disadvantaged populations. The cases were then repeated giving a brief social background of the patient. Study participants were asked to rank on a 5-point scale their interest level in caring for the patient as well as their level of empathy toward the patient. Results: Forty-seven (68\%) emergency physicians completed the 25 -item questionnaire. Thirty-five (75\%) respondents stated that at times they do not have the same amount of patience when interacting with disadvantaged populations. Thirty-one $(66 \%)$ stated that at times they look down upon patients who present with conditions related to their substance abuse. When case scenarios were presented and then repeated with the addition of traumatic social experiences, $32 \%$ of respondents stated that they would be more engaged when caring for the patient after understanding some of their social history, while $59 \%$ stated that their level of engagement would not change. When no apparent social traumatic event preceded the patient's substance abuse, $24 \%$ of respondents were less interested, while $65 \%$ of respondents did not change their level of engagement. Fifty percent of respondents also stated that when given a social background, their level of empathy for the patient increased. Conclusion: Thirty-two percent of physician respondents stated that interest in caring for patients from disadvantaged populations increased when they were made aware of prior traumatic social experiences. Physician empathy toward the patient also increased for $50 \%$ of respondents when a social history was given. 
P53 The use of antibiotics in corneal abrasion and corneal foreign bodies in the emergency department

M. Sharma, MD, CCFP(EM), S.L. McLeod, MSc, A. Shah, MD,

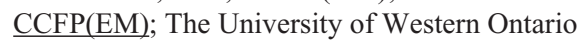

Keywords: antibiotic, corneal abrasion, emergency medicine, foreign body

Introduction: There continues to be debate over the utility of topical antibiotics in the emergency department (ED) management of corneal injury due to abrasion or foreign body. There is insufficient published literature to guide clinicians whether this practice is beneficial. Topical antibiotics can be expensive and may be associated with adverse events and allergic reactions. The objective of this study was to determine how many patients with corneal abrasion or foreign body were prescribed antibiotics and how many had an unplanned return visit to the ED within 2 weeks. Methods: A retrospective medical record review was conducted for a random sample of adult ( $\geq 18$ years) patients presenting to one of two EDs of an academic tertiary care centre (annual census 125,000) with corneal abrasion or corneal foreign body over a 1-year study period. Using a standardized data collection tool, trained research personnel extracted data such as triage level, topical analgesics given, narcotics, antibiotics prescribed, follow-up arranged, and unplanned return visit to the ED. Descriptive statistics were calculated using Microsoft Excel. Results: A total of 124 patients were included in this study. Sixty-three $(51 \%)$ patients had a corneal abrasion, and $61(49 \%)$ had a corneal foreign body. Foreign body type was metal in $38(62 \%)$ cases. Fifty-six (45\%) patients were prescribed topical antibiotics; the most common choices were Polytrim $(n=16)$ and Vigamox $(n=14)$. More patients with corneal abrasion were prescribed antibiotics compared to patients with foreign body $(56 \% \mathrm{v}$. $34 \%, \Delta 22 \% ; 95 \%$ CI: 4,37$)$. There was one unplanned return visit to the ED for pain. No patient in this sample developed infection, whether prescribed antibiotics or not. Conclusion: There is significant practice variability in the use of topical antibiotics post corneal injury. This retrospective review suggests that topical antibiotic use may not be necessary in this population

P54 Procedural sedation for cardioversion of atrial fibrillation in the emergency department: is it different to sedation for more painful procedures?

M. Butler, BSc, S.G. Campbell, MB BCh, P. Zed, PharmD, P. Froese, CCP, R. MacKinley, ACP, K.D. Magee, MD, FRCP, M. Watson, MD CCFP(EM), G.J. Kovacs, MD, FRCP; Charles V. Keating Emergency \& Trauma Centre

Keywords: atrial fibrillation, cardioversion, emergency medicine, procedural sedation

Introduction: Atrial fibrillation (AF) is the most common arrhythmia treated in the ED. The proportion of procedural sedations (EDPS) for cardioversion at the Halifax Infirmary ED increased from 6\% in 2006 to $13 \%$ in 2011 . Anecdotally, sedationists have observed differences in practice for EDPS for cardioversion compared to that for other reasons. This opinion would be important to verify objectively. Methods: This is a retrospective study of 3,015 records from the Halifax EDPS registry from January 22, 2006, to May 19, 2012. We compared clinical details between patients who received EDPS for cardioversion to those receiving PSA for other indications. Results: Patients in the AF group were less healthy at baseline (OR of ASA $1=0.47 ; 95 \%$ CI $0.37-$ 0.61 ), were $81 \%$ more likely to be male (OR $1.81 ; 95 \% \mathrm{CI}=1.41$ 2.32 ), and were on average 2.2 years older. The AF group received lower doses of propofol (average $0.055 \mathrm{mg} / \mathrm{kg} \times$ min propofol v. 0.105 $\mathrm{mg} / \mathrm{kg} \times \mathrm{min})(p=0.02 ; 0.31,4.08) ; 53.3 \%$ of $\mathrm{AF}$ patients received no analgesia versus $8.6 \%$ for comparators $(\mathrm{OR}=12.06 ; 95 \% \mathrm{CI}=9.20$,
15.82). Hypotension was more common in the AF group $(26.7 \% \mathrm{v}$. $11.4 \%)(\mathrm{OR}=2.51 ; 95 \% \mathrm{CI}=1.87-3.34)$, as was application of an airway intervention $(\mathrm{OR}=1.45 ; 95 \% \mathrm{CI}=1.08,1.93)$. AF patients reported satisfaction with the procedure in $37.1 \%$ versus $27.1 \%$ of comparators. $(\mathrm{OR}=0.63 ; 95 \% \mathrm{CI}=0.47-0.84)$. Conclusion: Patients with AF receiving EDPS for cardioversion had shorter durations of sedation than other EDPS patients, received lower doses of propofol, and had a higher incidence of adverse events and interventions and patients were less likely to report satisfaction with the procedure. It appears that patients needing cardioversion in the ED differ from other patients requiring EDPS and that EDPS for this group differs from that for other reasons. Further research will identify an optimal approach to EDPS for AF cardioversion.

P55 Assessing the feasibility of utilizing national emergency department quality indicators to evaluate stroke care in an Ontario Local Health Integrated Network

W. Supapol, PhD, M. Ackerman, MD, S. Kianpour, BSc, C. Tasch, BSc, A. Worster, MD; McMaster University

Keywords: emergency department, emergency medicine, quality indicator, stroke

Introduction: We applied 2 of ICES consensus list of 48 evidencebased quality indicators for Canadian emergency departments (EDs) to assess and compare the quality of stroke care at 12 Ontario EDs within a single Local Health Integrated Network (LHIN). Methods: We reviewed approximately 100 randomly selected charts with a diagnosis of stroke at each ED for adult visits occurring between July 2009 and June 2011. Our two trained data abstractors used standardized data collection procedures and forms, and data were simultaneously double abstracted and differences resolved by consensus. We examined all emergency medical service (EMS) and ED records for blood glucose by EMS or within 30 minutes of ED arrival and 12-lead electrocardiogram (ECG) by EMS or within 6 hours of ED arrival. Where times were missing, the indicator was not counted as being met. We determined the proportion of stroke visits where these indicators were met and $95 \%$ confidence intervals (95\% CI) and tested whether the observed proportion was significantly different from hypothetical standards. Results: All 12 hospitals agreed to participate, and we reviewed 1,126 ED stroke visit charts. EMS charts were missing in 7 to $42 \%$ of visits arriving by ambulance. Glucose testing was performed by EMS or in the ED within 30 minutes in 33 (95\% CI: $24-43)$ to $70 \%(60-79)$ of visits. Where glucose was not previously tested by EMS, glucose was tested in the ED in 76 to $98 \%$ of visits at 11 hospitals, with an average median time of 62 minutes. ECG tests were performed in over $80 \%$ of visits at most hospitals. Conclusion: Stroke patients are usually tested for glucose but frequently not within 30 minutes of arrival. ECG testing is close to the standard of $80 \%$ at all hospitals. Missing times, EMS and ECG sheets may result in underestimates of hospital performance.

P56 Validation of the clinical prediction rule for recurrent venous thromboembolism in cancer patients: the Ottawa score

Y. Kim, MD, S. Ahn, MD; Asan Medical Center, University of Ulsan, College of Medicine, Seoul, Korea

Keywords: cancer, emergency medicine, venous thromboembolism Introduction: Recently, a clinical prediction rule for recurrent venous thromboembolism in cancer patients, named the Ottawa score, was derived to individualize treatments with different intensities. It includes variables of sex, primary tumour site, tumour stage, and prior history of venous thromboembolism. The objective of this study was to validate the Ottawa score in an independent patient population in a ter- 
tiary hospital in Korea. Methods: Medical records of consecutive patients above 18 years old with active malignancy and newly diagnosed venous thromboembolism between January 1, 2006, and December 31,2010, were analyzed. Using the same definition of predictor variables in the original derivation study, patients were divided into low- (score $\leq 0$ ) and high- (score $\geq 1$ ) risk groups for recurrent venous thromboembolism, and their recurrence rates were calculated. Results: A total of $546(45.7 \%)$ patients were analyzed, and 99 (18.1\%) had recurrent venous thromboembolism during the follow-up period. In the low-risk group, 34 (13.2\%) had recurrent venous thromboembolism, compared to $65(22.4 \%)$ in the high-risk group. The Ottawa score performance showed $66 \%$ sensitivity, $50 \%$ specificity, $22 \%$ positive predictive value, and $87 \%$ negative predictive value in our validation cohort. Conclusion: We were not able to accurately ascertain the relevance of the Ottawa score in our validation cohort. Future validation studies including diverse patients with different cancer predominance are warranted.

P57 Handouts at emergency medicine continuing education and professional development (CEPD) events: are they worth the paper they're printed on?

R. Penciner, MD, MSc, CCFP(EM), FCFP; North York General Hospital

Keywords: education, emergency medicine, handouts

Introduction: Handouts for CEPD events are often an expectation of event organizers and participants. The utility of handouts in CEPD events is unknown. The purpose of this study was to determine participants' preferences for handouts and their perceived and real use of the handouts postevent. Methods: Emergency Medicine Update Europe was a continuing education conference in Monteriggioni, Italy, in September 2011. The conference consisted of 19 45-minute presentations over 5 days by leading North American faculty. All faculty provided handouts prior consisting of a maximum of four pages. A printed and electronic syllabus was provided at the beginning of the conference. Prior to the first session, registrants were invited to complete an anonymous paper six-item questionnaire. Six months following the conference, participants who completed the first survey were contacted by email to complete an online two-item questionnaire. Results: Fifty-eight registrants attended the conference, mostly from Canada. Thirty-five registrants completed the first survey $(\mathrm{RR}=$ $60 \%)$. Twenty-seven registrants completed the second survey $(\mathrm{RR}=$ $77 \%$ ). Fifty-four percent reported that they were likely or very likely to refer to the presentation handouts in the next 3 to 6 months. Six months later, $44 \%$ reported that they occasionally referred to the notes and $48 \%$ rarely or never referred to the notes. Sixty-eight percent referred to notes from 3/19 or fewer presentations. Paper notes were the preferred format $(54 \%)$. Sixty percent would rate the speaker lower on an evaluation if notes were not provided. Conclusion: Handouts at conferences have a perceived value by registrants but are often not referred to after the conference.

\section{P58 Emergency department follow-up for unattached patients C.E. Krook, BMedSci, P. Parks, MD; University of Alberta}

Keywords: continuity of care, emergency medicine, primary care Introduction: To describe and evaluate the effectiveness of an emergency department (ED) follow-up clinic for patients who present to the ED, who have no family doctor as a routine primary care provider, but who still require semiurgent follow-up for their medical complaint. Methods: The Emergency Department Unattached Follow-up Clinic (ED UFC) was opened in October 2011 at the Medicine Hat Regional Hospital as a pilot project. To evaluate the pilot project, the initial 6 months of data were collected and analyzed. The main outcome measures were patient satisfaction, admission rates, and ED visit rates. Results: Over the 6-month period, 102 patients were referred to the ED UFC; however, only 78 (76\%) patients were included in the data set. Patient satisfaction with their interaction with the clinic was reported as extremely high. ED physicians felt that the use of the ED UFC avoided 20 admissions to hospital $(20 \%$ reduction in need for admission), and only two of those patients were subsequently admitted within 90 days regardless of the initial presenting complaint. Rates of presentation to the ED dropped within the population from an average of 2.40 to 0.45 visits over 90 days pre and post clinic attendance. Overall, it is estimated that by preventing acute hospital admission for these patients, over $\$ 130,000$ was saved in administration and hospital costs over 6 months due to the establishment of the clinic. Conclusion: The establishment of the ED UFC provides an effective alternative for the follow-up of unattached patients who present to the ED and achieves high patient satisfaction. In addition, it reduces both admission to hospital as well as repeat ED visit rates. The clinic was created as a safety net for patients without access to primary care and may help improve health care access and continuity of care for unattached patients.

P59 Validating the McMaster Modular Assessment Program: an evidence-based, clinical assessment system

T.M. Chan, MD, J. Sherbino, MD, MEd, Y. Park, PhD, A. Kirkham, MD, A. Pardhan, MD, MBA, N. Del Bel, MD, C.F. Wallner, MD, T. Swoboda, MD, MS, B. Judge, MD; University of Illinois at Chicago

Keywords: assessment, education, emergency medicine, residency Introduction: The McMaster University Emergency Medicine program, with collaborators at the universities of Alberta and Saskatchewan, created the McMaster Modular Assessment Program (McMAP) for postgraduate medical education. We describe our method for setting standards and establishing content validity. Methods: The McMAP developers created 54 unique clinically based assessment instruments for junior and intermediate-level residents. To establish content validity, we sent the tools to a panel of six international reviewers (three Canadian and three American; two attendings and one resident per country) to sort the instruments by CanMEDs Role/ACGME Competency and expected level of achievement (junior, intermediate, or senior). Intraclass correlations (ICCs) were calculated to generate rater agreement indices. Results: While all reviewers found our portfolio comprehensively covered all CanMEDs Roles and ACGME Competencies, mapping each instrument to the appropriate Role or Competency led to disagreement. The overall ICCs for CanMEDS and ACGME competencies were 0.68 and 0.44 , respectively. Some ACGME domains in particular had low ICC: Patient Care (ICC $=0.27$ ), Teaching (Practice-based Learning and Improvement) (ICC = 0.26 ), and Professionalism ( ICC $=0.21)$. Using a modified Angoff method, we identified expected levels of achievement for each of the 54 instruments. After sorting by reviewers, the majority of instruments remained at their original levels of achievement $(50 / 54=93 \%)$. However, nearly half $(23 / 50=46 \%)$ of the instruments were also deemed useful at other levels (e.g., junior and intermediate levels). Conclusion: We present a novel method to establish content validity for an assessment program that incorporates a large number of instruments. Our findings suggest that individual physicians are consistent in sorting clinical assessment instruments to expected levels of achievement (i.e., junior, intermediate, senior), but they have difficulty mapping assessment instruments to the CanMEDs or ACGME competency frameworks. Our findings may inform areas for faculty development.

P60 COMMOTIO cordis managed with therapeutic hypothermia L. Murphy, MD, R. Green, MD; Dalhousie University 
Keywords: commotio cordis, critical care, emergency medicine, therapeutic hypothermia

Introduction: Therapeutic hypothermia $(\mathrm{TH})$ is currently utilized as a neuroprotective strategy for patients with persistent neurologic compromise following ROSC from cardiac arrest. The current ACLS guidelines recommend the use of TH for those patients with VF arrest. However, it is possible that TH improved patient outcomes in non-VF patients with anoxic brain injury, such as traumatic cardiac arrest. Reports describing TH use in non-VF cases may inform clinical practice. Methods: We describe a case of a patient who presented with cardiac arrest secondary to commotio cordis and was managed with $\mathrm{TH}$ An extensive literature review was performed on the use of $\mathrm{TH}$ in nonVF traumatic cardiac arrests. Commotio cordis is the term for cardiac arrest that occurs following a blunt, nonpenetrating blow to the chest. It often occurs in association with sports-related projectiles and is associated with an extremely high mortality rate. Results: Our patient was a 43-year-old male who suffered cardiac arrest after being struck in the chest with a baseball bat. He was defibrillated twice at the scene by paramedics and achieved ROSC. On arrival in the emergency department his GCS was 6, with a normal CT head. The patient was transferred to the ICU, where TH was initiated approximately 4 hours following the arrest. The target temperature of 32 to $34^{\circ} \mathrm{C}$ was maintained for 27 hours. The patient was extubated on postadmission day 3 and recovered to his baseline neurologic status. No previous evidence for the use of TH in commotio cordis has been described. Conclusion: TH is instituted routinely in the management of VF arrest. Our case illustrates the first described adult case to use $\mathrm{TH}$ in the management of commotio cordis.

P61 The role of pharmacists within the context of an emergency department-based adverse drug event screening program M. Quinn, MD, G.S. Hunte, MD, PhD, C.M. Hohl, MDCM, FRCP, MHSc; University of British Columbia

Keywords: adverse drug event screening, emergency medicine, patient safety, pharmacist

Introduction: Adverse drug events (ADEs), unintended and harmful events resulting from medications, are commonly not recognized in emergency departments (EDs). In 2011/2012, we implemented a twostep screening program in three EDs that consisted of (1) identification of high-risk patients using a clinical decision rule and (2) medication review in high-risk patients by clinical pharmacists to identify ADEs. Methods: In this prospective qualitative study, we observed ED pharmacists as they reviewed the medications of high-risk patients. ED pharmacists also participated in semistructured interviews that consisted of questions directed at the following areas: their role, workflow, patient assessments, communications, challenges of working in the $\mathrm{ED}$, and their perceived contributions. We audiorecorded interviews and transcribed them verbatim. A three-member analysis committee independently reviewed the transcripts and identified recurrent themes. Results: Pharmacists assessed patients through patient and family interviews and by reviewing charts, medication lists, and physician notes. Pharmacists often assessed patients before or in parallel to the emergency physicians and were asked to think about the patient independent of the physicians' assessments. Overall, pharmacists perceived this role as practice changing. Most were accustomed to anchoring their assessments to a physicians' diagnostic impression and found it difficult and often premature to make a judgment with uncertain or limited information. In addition, role, space, and time negotiations constrained integration within the ED. Communication was primarily asynchronous (chart documentation, fax), and only "valuable" diagnostic or treatment-altering information was conveyed to treating emergency physicians. Pharmacists saw their most important contributions as taking comprehensive medication histories, assessing patients for possible ADEs, and offering a complementary but different perspective. Conclusion: Pharmacists participating in an ADE screening program experienced their role as valuable but limited, identifying their most important contributions as providing comprehensive medication histories and identifying ADEs.

P62 The sensitivity and specificity of the focused assessment with sonography for trauma in blunt abdominal injury in a level 1 trauma centre: a retrospective study

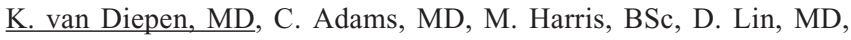
CCFP(EM); McMaster University

Keywords: emergency medicine, FAST, trauma

Introduction: The American College of Surgeons cites the sensitivity and specificity of the focused assessment with sonography for trauma (FAST) to be comparable to computed tomography (CT) scan. However, the literature-cited accuracy of FAST in blunt abdominal trauma (BAT) as well as local FAST utilization is (known to be) variable. This study examined the local sensitivity, specificity, and predictive values of FAST in BAT to aid knowledge translation and determine the need for quality assurance. Methods: The local trauma registry was accessed to identify patients with BAT who underwent FAST, abdominal CT, and/or laparotomy between 2004 and 2011. Ninety-nine charts were retrospectively reviewed. Demographic data (age, gender, injury mechanism, hemodynamic instability [systolic blood pressure $\leq$ $100 \mathrm{~mm} \mathrm{Hg}$ ], injury severity score) were coded along with FAST, CT, and operative results. A positive FAST required charted positive results, while a positive $\mathrm{CT}$ scan or operative report required a dictated record of intraperitoneal free fluid, irrespective of amount. All data were analyzed using SPSS software. Results: Of the 99 charts, 83 charts met inclusion criteria. The mean age was 40 years, and 62 patients had free fluid identified on CT scan or operative report. The local sensitivity, specificity, and positive and negative predictive values were $72.6 \%$ (95\% confidence interval 65.7-76.8), 81\% (60.6-93.4), 91.8 (83.197.2 ), and $50 \%$ (37.5-57.7), respectively. Hemodynamic instability was the only significant predictor of the need for operative intervention $(p=$ 0.027). In a subgroup analysis of unstable patients, the FAST's positive predictive value for procedural intervention was 81\% (69.4-90.5). Conclusion: Local sensitivity and specificity of FAST in BAT were low; however, positive FAST in hemodynamically unstable patients frequently predicted the need for procedural intervention. A prospective study is required to better assess the local characteristics of FAST. The local trauma program should consider a quality assurance program to maximize the utility of FAST in BAT.

P63 Electronic surveys to study return visits after ED care: a novel mechanism to better understand quality

T. Principi, MD, S.C. Porter, MD; The Hospital for Sick Children

Keywords: emergency medicine, patient satisfaction, questionnaire Introduction: Return visits after emergency department (ED) care serve as a measure of quality. However, important episodes of care occurring outside the index ED may be missed. Email-based survey mechanisms may afford an effective solution, but data are lacking with regard to the value of information captured via this approach. The objectives of the current study were to compare the proportion of patients seeking additional care beyond an index ED visit captured via an electronic survey versus documented at the index hospital and to evaluate the influence of additional care on self-reported satisfaction with ED care. Methods: We conducted a prospective observational study over a 4-week period to evaluate patient outcomes postdischarge from an urban, tertiary pediatric ED. Emails with a survey link approximately 1 week after the ED visit with up to two additional reminders. Patient information from each visit was extracted and merged with sur- 
vey data for evaluation. Results: A total of 1,030 (37\% of eligible visits) postvisit surveys were completed during the study period; 355 (35\%, 95\% CI 31-37) patients reported additional care after their index ED visit, and 291 (82\%, 95\% CI 78-86) sought care elsewhere. Five of $39(13 \%)$ subsequent hospital admissions were only captured through the survey. Patients who sought additional care were more likely to rate their overall care in the ED as fair/poor (OR 2.5, 95\% CI 1.3-4.8), feel they were not told what to watch for (OR 5.3, 95\% CI 2.7-10.2), and reported worse health (OR 14.9, 95\% CI 3.4-65.4). There no differences with respect to patient age, preferred language, or discharge diagnosis. Conclusion: Return visits are influenced by satisfaction with care, reinforcing the importance of capturing all repeated visits. Electronic surveys offer a scalable and cost-effective means toward accomplishing this goal.

\section{P64 Making sense of safety: a meta-narrative review}

G.S. Hunte, MD, PhD, J.J. Joyce, PhD, W.H. McKellin, PhD, S.B. Sheps, MD, MSc; St. Paul's Hospital

Keywords: emergency medicine, patient safety, systematic review Introduction: Patient safety is a complex and "wicked" problem. Despite a decade of considerable effort focused on reducing patient harm, it nevertheless remains a pervasive and persistent global health care issue. We sought to explore the meanings of "safety" within and across the health care and safety science literature. Methods: We conducted a systematic meta-narrative review to identify the meanings of safety within and across disciplinary boundaries. We applied multiple search strategies in the health care, organizational, safety science, and engineering literature and identified over 1,500 full-text sources where safety was conceptualized and studied in different ways by different communities of researchers. Results: Safety is most commonly conceptualized as freedom from injury or harm or unacceptable risk. In the traditional view, safety is achieved by avoiding what goes wrong (Safety I) and is improved by eliminating variation through automation, standardization, and routinization of best practices. Deviation from normative procedures, or error, is something to be categorized, counted, and reported. This normative view is predicated on positivistic and reductionistic assumptions that everyday work is based on repetitive (linear) processes. This is the dominant view in health care. However, a view of safety informed by complexity science and resilience engineering suggests otherwise. Recent developments in safety theory shift toward an understanding of safety as resilience achieved by facilitating everyday work and ensuring as much as possible goes right (Safety II). Safety, as social practice, is something a complex sociotechnical system does, a system phenomenon that emerges out of interactions in the presence of adaptive capacity to cope and sustain operational performance in the face of threat. Conclusion: Safe clinical practice cannot then simply be a question of eliminating risk for risk is inherent in health care. Rather, safety is dynamic, emerges from everyday practice, and is created within the interdependence among human and organizational actors, technological artifacts, and situated conversations.

P65 Diagnostic outcomes for patients presenting to the emergency department (ED) with chest pain

G. Innes, MD, E. Grafstein, MD, K.C. Innes, BSc, S. Stackhouse, BSN; University of Calgary

Keywords: chest pain, diagnosis, emergency, emergency medicine Introduction: Our objective was to describe the spectrum of discharge diagnoses in a consecutive cohort of ED patients with chest pain. Methods: This administrative database study included all patients presenting to six Vancouver Coastal Health (VCH) emer- gency departments with a chief complaint of chest pain. VCH sites use common triage codes and standardized triage training. Triage nurses are instructed to use the following chest pain categories: CV022 for patients with possible ACS, CV027-30 for other acute cardiovascular conditions, and CV023-26 for noncardiovascular conditions (e.g., GI, respiratory). Patients were stratified into three triage groups - CV022, CV027-30, CV 023-026 - and discharge diagnoses were collated for each group. Results: During a 6-month period, 13,721 patients presented with chest pain: 5,588 (41\%) in CV022, $1,371(10 \%)$ in CV027-30, and 6,762 (49\%) in CV023-26. In the CV022 (possible ACS) group, the mean age (SD) was 62.3 (15.7) and $56 \%$ were male. In this category, discharge diagnoses were chest pain NYD in $65.9 \%$, ACS in $13 \%$, dysrhythmias in $3.0 \%$, other cardiovascular conditions (CHF, angina, syncope, pericarditis, aortic dissection) in $3.6 \%$, respiratory conditions (pneumonia, PE, COPD, pneumonia, pneumothorax) in $3.8 \%$, GI in $4 \%$, and other (neurologic, musculoskeletal, anxiety or systemic) in $6.5 \%$. In the CV027-30 (other acute cardiovascular) triage group, the mean age was $61.1(16.6), 53 \%$ were male, and corresponding diagnostic proportions were $65.5 \%, 10.2 \%$, $2.5 \%, 5 \%, 5.9 \%, 3.5 \%$, and $7.4 \%$. In the CV023-26 (noncardiovascular) group, the mean age was $48.6(18.8), 53 \%$ were male, and diagnostic proportions were $65.2 \%, 1.7 \%, 0.5 \%, 1.6 \%, 7.7 \%, 5.4 \%$, and $17.9 \%$. Conclusion: Sex distribution was proportional across acuity groupings, suggesting no gender-based acuity bias. Most patients did not receive a specific explanatory diagnosis. Diagnostic mix was similar in ACS and non-ACS (other cardiovascular) triage groups and similar across the noncardiovascular groups, suggesting that triage distinguishes acuity but does not accurately differentiate ACS from other cardiovascular diagnoses.

\section{P66 Expectant parents' understanding of the implications and management of fever in the neonate \\ S.R. Ahronheim, MDCM, D. McGillivray, MDCM, FRCPC, S. Klam, MDCM, FRCSC, S. Barbic, MSc, BScOT, P. Brisebois, Medical Stu- dent, K. Lambrinakos-Raymond, Medical Student, J. Nemeth, MDCM, CCFP(EM); McGill University Health Centre}

Keywords: emergency medicine, fever, neonate

Introduction: The objective of this study was to evaluate expectant parents' knowledge of fever in the neonate aged 30 days or less and to discover whether they know to bring a febrile neonate for emergent medical assessment given the risk of serious bacterial infection (SBI). Methods: We conducted an anonymous survey of 355 expectant parents at the obstetrical ultrasound clinic of a diverse, urban tertiary care hospital in Montreal, Canada. The participants answered questions about fever in neonates, including whether they would bring the infant for immediate medical evaluation. Demographic data were collected. Results: Only $79 \%$ of parents reported that they would take a febrile neonate for immediate medical assessment, leaving a full fifth of the target population who might not be evaluated by a health care worker. Of a possible maximum score of 6 , the mean total knowledge score was $2.49 \pm 1.20(95 \%$ CI $2.39,2.62)$, or $41.5 \%$. Most participants could not identify why fever in the neonate is dangerous, and the majority could not define the oral or rectal temperature of fever in this age group. Level of education and immigrant status (whether the parent was born in Canada) were significant predictors of knowledge score. No demographic characteristics correlated with whether a parent would seek urgent medical care for a febrile neonate. Conclusion: The study results indicate that $21 \%$ of febrile neonates may not be brought for timely medical care, putting them at risk for development of SBI and for significant morbidity and mortality. With this study, we have identified an important area for further health education of expectant parents to improve the health of neonates. 
P67 Demographic characteristics and resource utilization of emergency department frequent users

M. Riggan, MD, S.L. McLeod, MSc, K. Fielding, BSc, A. Tran, BSc, D. Ouellette, MD, FRCPC; University of Western Ontario

Keywords: demographics, emergency department, frequent users Introduction: Frequent users of the emergency department (ED) are often patients with chronic, poorly controlled medical and psychiatric conditions. It is suspected that frequent users contribute significantly to total ED visits and resource utilization, potentially due to lack of access to primary care, social services, and other outpatient resources. The objectives were to explore the demographic characteristics of ED frequent users and assess usage of other medical services and resources during their ED visit. Methods: A retrospective medical record review was conducted for a random sample of adult $(\geq 18$ years) patients with $\geq 7 \mathrm{ED}$ visits to an academic tertiary care centre (annual census 125,000) from April 1, 2010, to March 31, 2011. Patients were excluded if they were a direct referral, the ED chart was unavailable, or they had a planned return ED visit. Between-group comparisons were made using a control group of nonfrequent users $(<$ 4 ED visits). Results: Seventy-nine nonfrequent users had 114 visits; 44 frequent users had 745 visits. Groups were similar in age, gender, and family physician status. ED LOS was similar between groups. Frequent users had fewer laboratory and imaging investigations compared to nonfrequent users, with the exception of an ethanol level (11.9\% v. $2.6 \% ; \Delta 9.3 \%$; $95 \%$ CI: $4.0,12.4)$. Respiratory, MSK, GI, psychiatric, and substance abuse conditions were higher $(p<0.05)$ in the frequent user group. Very few frequent users were referred for outpatient follow-up (2.8\%), social work $(2.7 \%)$, or CCAC $(4.2 \%)$. Frequent users were less likely to have any discharge planning documented on their ED record $(47.0 \%$ v. $73.7 \% ; \Delta 26.7 \% ; 95 \%$ CI: 17.2 , 34.7). Conclusion: Frequent users are a diverse patient population with more psychiatric and substance issues. Important medical and social services may be underutilized in their discharge planning from the ED. Future research should investigate if better utilization of these services would decrease ED usage.

P68 Hooked: fish hook removal in the emergency department D.K. Ting, BSc, R.J. Brison, MD, FRCPC; Queen's University

Keywords: emergency medicine, fishing, foreign body removal Introduction: Fishing is a recreational activity enjoyed throughout Canada. The removal of embedded fish hooks seems to be a procedure relished by many emergency department (ED) clinicians lucky enough to work in centres adjacent to recreational opportunities for fishing. There are few reports on the incidence of infection or the need for antibiotic prophylaxis after freshwater fish hook injuries. Our objective was to describe the occurrence of these injuries, how they were managed in the ED, and the likelihood of infection or other complications postremoval. Methods: Persons presenting to the ED during a 5-year period through 2010 were identified by searching an ongoing emergency department injury surveillance program that identifies all persons presenting to either of Kingston's two EDs with an acute injury. This program collects information to describe circumstances of the injury event and on the resulting anatomic injury. We abstracted information on subsequent ED-based care in the 30 days post ED encounter from the single electronic patient care system serving the two acute care hospital systems in our community of 150,000 . The primary outcome measure was the occurrence of an infectious complication post fish hook removal. This presumed that treatment for such would be sought in the ED. Information on measures of potential association included the anatomic location of injury, type of hook, use of anesthetic and irrigation, removal technique, gender, and age. Data are described using estimates of proportions. Results: There were 204 persons treated in our EDs for removal of embedded fish hooks over 5 years. There were none $(95 \% \mathrm{CI} ; 0,1.5)$ who returned for treatment of infection. The most frequent anatomic sites of injury were the fingers/thumb $(100 ; 49.3 \%)$, hand $(32 ; 15.8 \%)$, and head/face $(30$; $14.7 \%$ ); $74.3 \%$ had multiple or treble hooks embedded. Local anesthetic was recorded as used in $60 \%$ of cases, wound irrigation in $20.6 \%$, and antibiotic prophylaxis in $8.4 \%$. "Advance and cut" was the most often used removal technique $(38.2 \%)$ followed by "needle cover" $(27.0 \%)$ and entry-site removal (16.9\%). Eighty-four percent of patients were male, distributed evenly across age groups. Eighty-three percent had hooked themselves (of which none got away!). Conclusion: While based on a moderate-sized, single-centre sample, this study suggests that there is no need for the use of antibiotic prophylaxis following freshwater fish hook removal.

P69 An expedited oral contrast protocol for suspected appendicitis does not lead to missed cases requiring surgery

P. Lalli, BSc, J. Gill, BSc, D. Wang, PhD, L. Mercuur, MD, E. Lang, MD, A. McRae, MD; University of Alberta

Keywords: appendicitis, computed tomography, emergency medicine Introduction: Appendicitis is a condition often seen in the emergency department (ED), but heavy reliance on $\mathrm{CT}$ and use of oral contrast lead to longer patient length of stay, vomiting, aspiration, and complications from delays in diagnosis. Many $\mathrm{CT}$ imaging protocols require a 2-hour, $1.5 \mathrm{~L}$ oral contrast load to visualize the bowel. We developed a 1-hour expedited contrast protocol to accurately identify suspected cases of appendicitis requiring surgery while confidently excluding normals. This study examined the safety of an expedited 1hour oral contrast CT protocol compared to the standard 2-hour prep. Methods: The study included all patients discharged from three urban adult EDs after undergoing contrast CT for appendicitis before (November 2008 to April 2009) and after (November 2011 to April 2012) implementation of a 1-hour expedited oral contrast protocol. Primary outcome was unplanned patient ED revisits as a surrogate for missed cases of appendicitis; secondary outcomes included repeat CT examinations and ultrasound examinations. Proportions were compared using Pearson chi-square and Fisher exact tests. Results: A total of 646 patients were included in the study; 320 patients underwent the 2-hour "pre" protocol, while 326 patients received the 1-hour expedited "post" protocol. Patient groups were similar with respect to age, gender, and Canadian Triage and Acuity Scale (CTAS). There was no difference in revisit rate at the 3 -day $(0.3 \%$ pre and post, $p=\mathrm{NS})$ or 14 -day $(0.6 \%$ pre, $0.3 \%$ post, $p=$ NS $)$ time point. The proportion of patients requiring repeat CT scans was similar $(0.3 \%$ pre, $0.9 \%$ post, $p$ $=\mathrm{NS})$. An increase in the number of ultrasounds performed was observed ( 30 pre, 53 post). However, $83 \%$ of ultrasound investigations were performed before CT imaging, suggesting a change in physician preference for this modality rather than as a product of the expedited protocol. Conclusion: A 1-hour expedited oral contrast CT protocol for suspected appendicitis appears as safe as a standard 2hour protocol, with no difference in missed appendicitis cases or need for additional CT imaging.

$P 70$ Stroke education in emergency medicine residency programs D.R. Harris, MD, MHSc, PhD (c), P. Teal, MD, S. Pulfrey, MD, B. Lahiffe, MD; Department of Emergency Medicine, St. Paul's Hospital

Keywords: cerebrovascular disorders, emergency medicine, medical education

Introduction: Stroke and transient ischemic attack (TIA) are common disorders cared for by emergency physicians. In the emergency 
department (ED), the diagnosis and management of these conditions are time sensitive and complex, requiring that emergency physicians have adequate training. This study sought to determine the extent of stroke education in Canadian emergency medicine residency programs. Methods: A two-page survey was emailed to directors of all English-speaking emergency residency programs in Canada in 2011. The number and types of required and elective rotations, lectures, and training in stroke, general neurology, and neuroradiology were assessed. Means, standard deviations (SD), and proportions (with $95 \%$ confidence intervals) are used to describe the data. Results: Sixteen responses were received, representing 20 programs (response rate $20 / 29=69 \%$ ). A mandatory rotation in general neurology was required in only $3 / 20(15 \%$; 95\% CI 5.2-36) and for stroke neurology, only $2 / 20(10 \%$; $95 \%$ CI $2.1-30.8)$. Electives in general or stroke neurology were offered in $5 / 20$ programs ( $25 \%$; $95 \%$ CI $11.2-46.9)$. Only $1 / 20$ programs had a clinical rotation in radiology or neuroradiology (5\%; 95\% CI 0.9-23.6). Programs had a mean (SD) of 160 (78) hours of didactic lectures per year, with a mean (SD) of 3 (1.2) hours devoted to stroke and TIA content, annually. Directors reported that 16/20 (80\%; 95\% CI 58.4-91.9) programs had practice oral examinations on acute stroke management. Of note, all 20 programs $(100 \%$; 95\% CI 83.9-100) trained residents in hospitals that administer thrombolytics for acute ischemic stroke. In contrast, 19/20 sites (95\%; 95\% CI 76.4-99.1) had mandatory rotations in cardiology. Conclusion: Currently, formal training in stroke in Canadian emergency medicine residency programs is limited. Enhancing training opportunities should be explored.

P71 The need for expanded point-of-care ultrasound training in rural emergency departments across Newfoundland and Labrador Z. Warren, Student, Doctor of Medicine Program, A. Smith, MD; Memorial University of Newfoundland

Keywords: emergency medicine, rural, ultrasound

Introduction: Point-of-care ultrasound (PoCUS) is a valuable tool in the practice of emergency medicine, particularly in rural locations with limited access to formal imaging. Access, level of training, and perceived importance of PoCUS across tertiary, category A (24-hour service), and category B (call back) emergency departments in Newfoundland and Labrador (NL) are currently unknown and sought to be addressed by this survey. Methods: A questionnaire was mailed to physicians practicing emergency medicine in NL seeking to characterize access, utilization, and interest pertaining to PoCUS. Results: The response rate was $43 \%(88 / 204)$, with a well-distributed response from tertiary, category A, and category B emergency departments. PoCUS is available in the adult St. John's emergency departments and in a majority of category A emergency departments, with minimal availability in category B departments $(93 \%$ v. $73 \%$ v. $4 \% ; p<0.05)$. Physicians from tertiary centres are more likely to have completed formal training in PoCUS when compared to category A and category B respondents $(93 \%$ v. $24 \%$ v. $0 \% ; p<0.05)$, with a similar number describing perceived competence in PoCUS (93\% v. 16\% v. $4 \%$; $p<$ $0.05)$. Overall, $98.8 \%$ believe that PoCUS is very or somewhat important to the practice of emergency medicine and $94 \%$ are very or somewhat interested in additional training. Conclusion: There is significant interest in PoCUS training throughout the province of NL, with appropriate equipment being available in a majority of category A emergency departments. PoCUS is considered to be important to the practice of emergency medicine, and formal training correlates well with self-assessed competence.

P72 Identifying chronic diseases in the ED: a pilot screening intervention for asymptomatic hypertension
J. Riley, MD, M. McGowan, MHK, K. Woolny, BScN, L. Morrison, MSc, MD, FRCPC; St. Michael's Hospital

Keywords: emergency medicine, hypertension, incidental findings, screening program

Introduction: Asymptomatic hypertension (HTN) is ubiquitous with the aging, obese Canadian population and, left untreated, holds serious risk for cardiovascular, renal, and cerebrovascular disease. While the emergency department (ED) functions to identify and manage an acute medical complaint, there presents an unexplored opportunity to screen and identify patients for chronic disease. Methods: In an academic, inner-city, level 1 trauma centre, patients $\geq 18$ years of age with asymptomatic blood pressure (BP) readings $\geq 140$ systolic or $\geq 90$ diastolic at two time points during an ED visit were eligible for inclusion. Those with a history of HTN, past or current use of HTN medication, unstable condition, incapacity to consent, and missed and declined participation were excluded. Clinical-demographic and administrative data were collected. The intervention was a letter with the issue of concern addressed to the family physician (FP) given to consented patients at ED discharge with encouragement to seek a follow-up visit with their FP. The primary outcome measure was completed follow-up with the FP at 6 weeks post ED discharge and number of new HTN-confirmed cases. Results: A convenience sample across weekdays, weekends, days, evenings, and night shifts resulted in the recruitment of 73 consenting patients (mean [SD] age $47 \pm 16$ years; $63 \%$ identified male gender; $63 \%$ housed v. $19 \%$ sheltered/homeless; $60 \%$ Canadian Triage Acuity Scale [CTAS] 3, 25\% CTAS 2; median 10 -point pain score $4 \pm 3$ ). Of these, $67 \%$ had $\geq 140$ systolic and $67 \%$ had $\geq 90$ diastolic BP readings and $34 \%$ had both. Almost a third were overweight (33\%) by BMI classification and $40 \%$ had a family history of HTN. Only a few had other risk factors: $19 \%$ high cholesterol; $7 \%$ endocrine disease; $5 \%$ TIA or stroke; $3 \%$ CAD or MI; and $86 \%$ reported being screened previously for HTN in last 2 years, yet only $12 \%$ had been advised to change their lifestyle (diet, exercise). Eightysix percent (51) of FPs were reached at follow-up confirming that $61 \%$ (31) patients had been seen and 4 still had high BP, were diagnosed with HTN, and were treated. Conclusion: A pilot, single-site study has demonstrated that measuring BP and reporting the asymptomatic, incidental finding of HTN while in the context of an unrelated acute medical visit to the ED hold the potential to identify new chronic disease, as confirmed by FPs.

P73 Three cases of neoplastic meningitis misdiagnosed as infectious meningitis in the emergency department

J. Shin, MD, S. Ahn, MD; Asan Medical Center, University of Ulsan, College of Medicine, Seoul, Korea

Keywords: cancer, cerebrospinal fluid, emergency medicine, neoplastic meningitis

Introduction: Neoplastic meningitis (NM) is diagnosed by neuroimaging or the presence of malignant cells in the cerebrospinal fluid (CSF). However, the sensitivity of the initial CSF and magnetic resonance imaging (MRI) of brain is low. Methods: We report three patients without known malignancy whose initial presentation was NM but were misdiagnosed as infectious meningitis in the emergency department (ED). Results: Case 1: A 68-year-old man visited our ED with a 3-month history of headache. With brain MRI and CSF study, he was diagnosed as tuberculous meningitis. After 20 days, repeated CSF cytology showed malignant cells. The final diagnosis was lung cancer with NM. He expired 10 weeks later. Case 2: A 57-year-old man visited a regional hospital ED with a 3-week history of headache, vomiting, and diplopia. Brain MRI showed no significant finding. With CSF examination, his diagnosis was aseptic meningitis. After a week of worsening headache, he was referred to our ED. Repeated 
lumbar puncture showed $80 \%$ of malignant cells. With endoscopy, he was diagnosed as stomach cancer with NM. He died after 8 weeks. Case 3: A 75-year-old man visited a regional hospital ED with headache lasting 4 months. His diagnosis was sinusitis. Persistent symptom brought him back to the same ED, and he developed recurrent generalized seizure. Brain MRI showed diffuse leptomeningeal enhancement suggesting meningitis, and he was transferred to our ED. CSF examination showed 4\% malignant cells. He was diagnosed as NM with unknown primary focus. He died after 4 weeks. Conclusion: Due to limitations of diagnostic modalities, diagnosing NM can be challenging, especially when the patient has no known history of malignancies. Repeated CSF study might not be a routine workup in the ED. However, when evaluating patients with headache, and considering its grave prognosis, NM should be kept in mind as a differential diagnosis of meningitis.

P74 Clinical impact of diagnostic imaging discrepancy and reinterpretation in an urban teaching hospital emergency department E.M. Merman, BASc, S.M. Friedman, MD, MPH, Amit Chopra, BSc; University Health Network, Toronto

Keywords: emergency medicine, error, imaging, safety

Introduction: We sought to characterize clinically significant diagnostic imaging (DI) discrepancies by radiology trainees and the impact on emergency department (ED) patients. Methods: Case series over a 6month period in a downtown teaching hospital. Emergency physicians (EPs) were recruited to flag discrepant DI reports deemed clinically significant. Cases were characterized using chart review and standardized EP interview. Results: Twenty-eight discrepant reports were identified (representing $0.1 \%$ of 18,185 images interpreted). The mean time between provisional discrepant diagnosis (PDDx) and revised diagnosis $(\mathrm{RDx})$ by attending radiology staff was 8.6 hours (median 4.8 hours, range $1.1,48.4)$, and $67.9 \%(n=19)$ of the patients had left the ED by the time of notification. The most frequently reported PDDx was CT abdomen/pelvis $(32.1 \%, n=9)$ and CT head $(28.6 \%, n=8)$. The impact of RDx was deemed major in $57.1 \%(n=16)$ for reasons including altered admitting status $(32.1 \%, n=9)$, immediate subspecialty referral $(n=16,57.1 \%)$, impact on management $(25 \%, n=7)$, and surgical management $(21.4 \%, n=6)$. EPs reported the likely impact of revised diagnosis as resulting in increased pain $(17.9 \%, n=$ 5), morbidity $(10.7 \%, n=3)$, and prolonged hospitalization $(25 \%, n=$ 7) but not altered long-term outcome or mortality. Conclusion: Relatively few clinically important discrepant reads were reported. RDx was associated with major clinical impact in $57.1 \%$ of reported cases, but few patients overall experienced increased morbidity, and no increased mortality was described. The importance of expedient communication of discrepant reports by staff radiologists is stressed, as is EP verification of patient contact information prior to discharge.

P75 What electrocardiogram (ECG) diagnoses do residents in emergency medicine need to know? Factoring in the opinion of the cardiology consultant

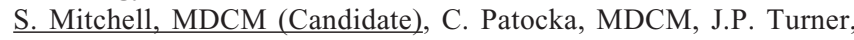
MDCM, MSc, FRCP, J. Wiseman, MDCM, MA(Ed), FRCP; McGill University

Keywords: ECG, emergency medicine, teaching

Introduction: Emergency physicians (EP) and cardiologists routinely use the ECG in patient-related decision making. We previously identified and categorized ECG diagnoses from an emergency medicine (EM) training perspective; however, the resulting pool of diagnoses was too general to allow us to develop a focused training program. Given that cardiologists and EPs have overlapping work domains, our study sought to determine whether the concordant agreement of EPs and cardiolo- gists could prioritize ECG diagnoses important for EM trainees, and this in turn could become the blueprint for an ECG curriculum in EM. Methods: We previously conducted a Delphi process using experts in EM. This current study involved an identical three-round Delphi process distributed to a purposeful sample of clinical cardiologists. The cardiologists assessed 121 ECG diagnoses and rated their importance for identification by an EM trainee. Consensus was defined as a minimum of $75 \%$ agreement, and stability was defined as a shift of $20 \%$ or less after successive rounds. Concordant diagnoses were those rated by both EPs and cardiologists as "must know" diagnoses. Results: Ten cardiologists participated in the Delphi and eight completed the process. Of the 121 diagnoses, 84 achieved consensus, 22 achieved stability, and 15 achieved neither consensus nor stability. Out of potentially important ECG diagnoses, 63 (52\%) were considered "must know" diagnoses, 41 (34\%) "should know" diagnoses, and 17 (14\%) "nice to know" diagnoses. There were 45 concordant "must know" diagnoses, while 25 "must know" diagnoses were discordant. Conclusion: We have solicited the opinion of two stakeholders in the management of cardiovascular emergencies to categorize which ECG diagnoses are most important for EM trainees to identify. This could allow clinical EM teachers to establish educational priorities. Specifically, the 45 concordant "must know" diagnoses will facilitate the initial development of an EM ECG training program and/or high-stakes evaluation.

\section{P76 Needs assessment survey of homeless and/or substance-using adults presenting to the emergency department}

K. Dong, MD, MSc, FRCP, R. Cooper, MD, MPH, FRCP, G. Salvalaggio, $\mathrm{MD}, \mathrm{MSc}, \mathrm{CCFP}, \mathrm{A}$. Newton, $\mathrm{PhD}, \mathrm{RN}, \mathrm{T}$. Wild, $\mathrm{PhD}, \mathrm{C}$. Vandenberghe, MSc, S. Kirkland, BSc, MSc, C. Villa-Roel, MD, PhD (Candidate), C. McCabe, PhD, B.H. Rowe, MD, MSc, CCFP(EM); Department of Emergency Medicine, Faculty of Medicine \& Dentistry, University of Alberta

Keywords: addiction, emergency department, emergency medicine, homelessness

Introduction: Individuals with unstable housing and/or active substance use issues are frequent users of emergency department (ED) services, yet little is known about how to best meet the needs of this highrisk group. The objective of this survey was to characterize these ED patients and assess the need for additional ED services. Methods: Patients presenting during study shifts at a large, urban ED were eligible for enrolment if they (1) had no permanent place of residence for the past 30 days and/or (2) if their chief complaint was related to substance use. Study shifts were randomly assigned to all hours and days of the week; consecutive eligible patients were approached for enrolment. Consenting patients verbally completed a survey documenting baseline characteristics and the need for additional services in the ED. Controls were enrolled in a 1:4 ratio. The analysis was descriptive, and comparisons between groups were done using chi-square or Fisher exact test. Results: A total of 209 patients (including 41 controls) were enrolled. The mean age of all participants was $42.8(\mathrm{SD}=14.3)$ years, and $136(65 \%)$ were male. The most commonly used drugs by target participants were tobacco $(128,78 \%)$ and alcohol $(118,72 \%) ; 104$ (63\%) were trying to reduce their alcohol consumption, and 73 (45\%) were trying to reduce their drug use. Target participants were more likely to report safety concerns as a reason for presentation $(p<0.05)$. Target respondents were interested in accessing assistance with housing $(109,68 \%)$, an addictions counselor $(106,67 \%)$, and a mental health counselor $(91,57 \%)$ in the ED. Conclusion: Patients without stable housing and/or those with substance use issues are interested in and willing to access additional addiction, mental health, and housing support services in the ED. Additional ED services should be offered and tailored to meet the unique needs of this population. 
P77 ACE-induced angioedema in the emergency department: an observational study

R. Curtis, BScH, S.L. Felder, BScH, R. Borici-Mazi, MD, I.M. Ball, MD; Queen's University Department of Emergency Medicine

Keywords: adverse drug reaction, angioedema, emergency medicine Introduction: Upper airway angioedema is a life-threatening emergency department (ED) presentation with several etiologies. Angiotensin-converting enzyme inhibitor (ACE-I)-induced angioedema (AAE) is increasing in frequency as the use of this medication class increases. The objectives of this study were to describe the incidence and management of AAE in the ED and to identify any epinephrine-induced morbidity. Methods: We conducted a retrospective chart review of consecutive angioedema patients presenting to two Canadian tertiary care EDs between July 2007 and March 2012 Data were collected using a structured extraction form. Medical records were excluded from the study if visible swelling was not present on examination or was secondary to local trauma, nonsystemic insect bite, or irritant exposure. Results: Of 1,702 medical records screened, 527 met both inclusion and exclusion criteria. The probable cause was identified in $48.8 \%(n=257)$. The most common identifiable etiology was AAE $(33.1 \%, n=85)$, with a $60.0 \%$ male predominance. The most commonly used medication was diphenhydramine $(63.5 \%, n=54)$, followed by corticosteroids $(50.6 \%, n=43)$ and ranitidine $(31.8 \%, n=27)$. Epinephrine was administered in $21.2 \%(n=$ 18 ) of AAE patients, 5 of whom received repeated doses. Epinephrine induced significant morbidity in two patients, causing myocardial ischemia or dysrhythmia shortly after administration. Four AAE patients required admission (4.7\%); one required endotracheal intubation. There was no associated mortality. Conclusion: Our study demonstrates that AAE is the most common identifiable etiology of angioedema. Routine management of AAE includes antihistamines and corticosteroids. Concerningly, epinephrine use is common. Many patients are started on an ACE-I for cardioprotection post-myocardial infarct. There is little physiologic rationale for epinephrine use in $\mathrm{AAE}$ and much risk. More work is necessary to better delineate epinephrine's role in this vulnerable patient population.

P78 Medical students' perceptions of novel remedial projects for emergency medicine multiple-choice question exam failures

C. Rabuka, MD, R.D. Lee, MD, P. San Agustin, MD, L. Evered, MD; University of Alberta

Keywords: emergency medicine, multiple-choice question exam, remedial, remediation

Introduction: No standard remediation process exists to help medical students who fail a multiple-choice question exam (MCQE). Based on each student's individual areas of weakness, we designed, implemented, and evaluated novel remedial projects (RPs) that targeted failed students' individual educational needs. Methods: After evaluating each student's specific areas of weakness, emergency physicans with undergraduate medical education expertise developed the RPs. The RPs were administered to 17 students who failed an emergency medicine rotation MCQE. To evaluate the students' perceptions of and satisfation with the RP process, a 7-point Likert scale questionnaire (developed using the Delphi method) was administered to these students. Results: Fourteen of 17 students completed the questionaire post-RP. Fourteen of 14 $(100 \%)$ agreed that (1) the RP motivated them to improve their knowledge; (2) they were satisfied with their level of knowledge at graduation; and (3) their supervising physician created an RP specific to their needs. Responses were mixed on whether the supervising physician took time to review the RP after completion, if the RP was a fair form of evaluating their weaknesses, and if they would have preferred a dif- ferent form of remediation. Conclusion: RPs are feasible to administer and allow students who failed a MCQE to achieve emergency rotation objectives. Not all students preferred this form of remediation, possibly due to its novel nature. One limitation is that a RP requires active participation by a supervising physician.

P79 A retrospective review of the use of a strep throat checklist allowing patient discharge from triage in a moderate-sized community emergency department

A. Keeler, MD, CCFP(EM), S.L. McLeod, MSc, M. Mann, MD, FRCPC; The University of Western Ontario

Keywords: emergency medicine, medical directive, streptococcal pharyngitis, triage

Introduction: The McIsaac scoring system aims to reduce inappropriate antibiotic prescriptions for pharyngitis and has recently been implemented as a medical directive in a community ED. Based on the patient's age and specific signs and symptoms, a score is given from 0 to 5. Patients with a score of 0 to 1 are given a symptomatic treatment handout and follow-up instructions and discharged from triage. Patients with a score of 2 to 3 are given the same handout, and a throat swab should be taken by the triage nurse before the patient is discharged directly from triage. Finally, those with a score of 4 to 5 should have a throat swab taken by the triage nurse and asked to stay to be seen by an emergency physician. The primary objectives were to determine if triage nurses were applying the medical directive and how many patients were discharged without seeing a physician. Methods: A retrospective chart review was conducted for a random sample of patients $\geq 4$ years of age who presented to a community ED (annual census 26,000) with a complaint of "sore throat" and were triaged as CTAS 4 or 5. Results: A total of 147 charts were included. The medical directive was implemented in $31(21.1 \%)$ cases. Twenty-three $(74.2 \%)$ of the screened patients had a strep score of $\leq 3$, indicating that they could be discharged without being seen by a physician; however, $10(43.4 \%)$ patients requested to see a physician. Median (IQR) ED length of stay (LOS) was $11(10,16.5)$ minutes for patients who were discharged home, compared to $105(67,169)$ minutes for patients who saw a physician. When the medical directive was applied, only one $(3.2 \%)$ patient received unnecessary antibiotics. This was a patient who could have been discharged home but requested to see a physician. Of the 38 patients who were seen by a physician and cultured (without initial application of the medical directive), 9 (23.7\%) patients received unnecessary antibiotics. Two patients who were not seen by a physician had an unplanned return visit to the ED within 7 days (both had negative cultures), compared to four patients who were seen by a physician. Conclusion: When the medical directive was applied, unnecessary antibiotic prescriptions were reduced, ED LOS was dramatically decreased, and there was no difference in the proportion of unplanned return visits to the ED. Future research should investigate barriers to implementation of this medical directive.

P80 Diagnostic test characteristics of a point-of-care test for HIV1 and $\mathbf{- 2}$ antibody in the emergency department

R. Stenstrom, MD, PhD, E. Grafstein, MD, I. Poureslami, PhD, F. Osati, PhD, F.X. Scheuermeyer, MD, D. Harris, MD, PhD, G. Hunte, $\mathrm{MD}, \mathrm{PhD}$, S. O'Donnell, MD, MSc, K. Rich, MD, MSc, K. Nemethy, MD, MSc; St. Paul's Hospital

Keywords: diagnostic test, emergency medicine, HIV

Introduction: Since 2006, on the recommendation of the CDC, many emergency departments (EDs) in the US have adopted HIV screening. Canada has not followed suit. Lab testing for HIV can take up to 10 days, making point-of-care (POC) testing relevant. Methods: We 
hypothesized that the diagnostic test characteristics would be excellent. Tobjewas to estimate the sensitivity, specificity, and predictive values for the INSTITM HIV-1/HIV-2 POC antibody test in an emergency department setting. This was a prospective, IRB-approved cohort study performed at St. Paul's Hospital ED between August 2009 and January 2011. ED patients were enrolled using probabilistic sampling of times of day/night and days of the week, based on patient volumes. Inclusion Criteria: Age 19 to 75 years of age and able to understand/provide informed consent. Exclusion Criteria: Intoxicated or in extremis. Subjects' HIV antibody status was obtained using the Biolytical INSTI POC test. All subjects with a positive POC test had confirmatory HIV antibody testing using Western blot as the gold standard. Subjects with negative POC tests who were having blood tests for clinical reasons also had confirmatory testing. Results: A total of 185 of 220 patients approached (follow-up $=84.1 \%$ ) were tested for HIV antibody with the POC test and Western blot. The mean age of participants was 45.6 years, and $65 \%$ were male. Sensitivity was $100 \%(65 / 65 ; 95 \%$ CI $99.2-100)$, specificity was $99.2 \%$ (119/120; 95\% CI 97.3-100), positive predictive value was $98.5 \%$ (95\% CI 94.8-100), and negative predictive value was $100 \%$ (95\% CI 99.6-100). Conclusion: A rapid POC test for HIV-1 and -2 antibody has high sensitivity, specificity, and positive/negative predictive values. The test results are available in less than 5 minutes, and this test would have utility in ED settings.

P81 Comparison of traditional triage scores and CTAS methods with a quick-look triage approach

M. Betz, MD, FRCP, J. Stempien, BSc, MD, CCFP(EM), FCFP, A. Wilde; University of Saskatchewan

Keywords: emergency medicine, quality improvement, triage, waiting time

Introduction: In an attempt to address long wait times for patients seeking medical attention in the emergency department, we investigated the possibility of introducing a rapid triage process based solely on chief complaint and the nurse's first impression of the patient. If implemented, it would minimize time spent at the triage desk and reduce patient line-ups. Methods: The student investigator was near the triage nurse as patients were being assessed. The nurse would give a rapid score to the investigator based on meeting the patient and hearing the chief complaint. Then a comprehensive CTAS score was assigned to the patient as usual. The analysis compared the accuracy of the rapid score the CTAS score. Results: In total, 496 patients were observed. Weighted kappa values could be individually calculated for 24 nurses. "Good" or "very good" measures of kappa agreement were achieved in $83.3 \%$. This indicates that the rapid score they gave after the chief complaint closely matched the CTAS score arrived at after a longer and more comprehensive process. Of the 496 pairs of observations, $419(84.5 \%)$ agreed. Concerning mismatches occurred in 13 patients $(2.6 \%)$. Of these 13,2 patients rated rapidly as 4 initially were subsequently given a CTAS level 2, while 11 were assessed as 3 on rapid scoring and then also upgraded to 2 . Further analysis revealed that triage experience had no effect on the degree of agreement. Conclusion: Triage nurses have the ability to triage patients based on a rapid assessment composed of a quick observation of the patient and chief complaint with little loss of accuracy compared to a complete triage nursing assessment. This may have implications for improving patient waiting times.

P82 Epidemiology of prehospital emergency responses for older adults in a provincial emergency medical services system

J. Goldstein, MS, A. Carter, MD, K. Rockwood, MD, J.L. Jensen, ACP

MAHSR, A. Travers, MSc, MD; Dalhousie
Keywords: aged, emergency medicine, EMS

Introduction: Societal aging is expected to have an impact on the use of emergency medical services (EMS). Older adults are known as high users of EMS. The objective of this study was to quantify the rate of EMS use in a Canadian provincial EMS system. Methods: A secondary data analysis of a provincial EMS data warehouse was conducted for emergency responses between January 1 and December 31, 2010. All older adults (aged 65+ years) requesting EMS for an emergent call were considered. EMS use in relation to age, gender, and geographic location was described. Results: During the study period, there were 30,653 requests for service. The mean age was $79.9 \pm 8.5$ years, and over half were women (57.3\%). The median Canadian Triage and Acuity Scale (CTAS) score was 3 (range 1-5). The overall response rate for older adults in this provincial EMS system was 202.8 responses per 1,000 , with a transport rate of 174.8 per 1,000 and a nontransport rate of 24.4 per 1,000. Conclusion: In this provincial EMS system, there was a high rate of EMS use among older adults. The rate of EMS use increased with age and was higher for women and in urban regions. The frequency of EMS use suggests the need for policies and clinical practice guidelines that reflect the unique needs of this demographic.

\section{P83 Evaluation of a hybrid simulation course for acquisition and} maintenance of emergency medicine procedures

A. Khazei, MD, G. Pachev, PhD, A. Mohamed, MD; University of British Columbia

Keywords: emergency medicine, knowledge translation, medical simulation

Introduction: The purpose of this study is to assess the short- and long-term effects of a simulation-assisted emergency medicine procedures course (SEMP). The course is unique in combining Web-based distance learning, animal parts task-trainers, and high-fidelity human patient simulators. It starts with an 8-hour Web-based modular curriculum providing background information and procedural animations. This block is combined with a 7-hour-long small-group hands-on procedural training program with participants practicing procedures on animal parts, synthetic task trainers, and integrating procedures into the overall care of a critically ill human patient simulator. Methods: Data records of participants from 12 administrations of the course over 3 years comprised the data set for the study. Participants were asked to complete surveys tapping their self-assessment of confidence in performing the procedures targeted in the course. The survey was administered at the beginning of the course, at the end of the course, and 3 months after the last group completed the course. Thus, the delay of the final administration varied for participants from 3 months to 3 years. Results: Data are currently being processed. Preliminary results indicate that participants rated this course favourably. There is an increase in confidence from pre- to immediate posttest. Confidence in procedure performance had different dynamics over time for the individual procedures. Conclusion: Overall, an emergency medicine procedural training curriculum utilizing a mixed-simulation model is effective at increasing participant's comfort and willingness to perform rare and new procedures. The different dynamics of confidence in one's procedural skills will be discussed in terms of opportunities for practice of the procedure, characteristics of the physician (experience, position, placement), and characteristics of the specific skill sets engaged in performing the procedures.

P84 Does the unscheduled return emergency department visit rate differ between patients treated by a resident versus a consultant? C. Rivest, MD, CCFP, S.L. McLeod, MSc, W. Millard, MD, FRCPC; The University of Western Ontario 
Keywords: bounce backs, emergency medicine, residents, unscheduled visits

Introduction: Resident supervision in the emergency department (ED) varies based on experience, program, and practice of the supervising consultant. Unscheduled return visits to the ED may represent an evolution of the presenting complaint or may reflect inadequacies in patient care at the initial visit. If the rate of unscheduled return visits is significantly different for patients treated primarily by a resident, it could suggest that enhanced supervision is necessary to ensure patient safety. The primary objective of this study was to determine the difference in the proportion of unscheduled return visits to the ED between patients treated primarily by a resident versus a consultant. Methods: A retrospective medical record review was conducted for a random sample of adult ( $\geq 18$ years) patients presenting to an academic tertiary care centre with two EDs (annual census 125,000) with an unscheduled return visit to the ED for a similar complaint within 7 days. Patients were excluded if they left against medical advice or before being seen and if the return visit was for an unrelated problem, direct referral, or imaging or if the patient was a frequent user of the $\mathrm{ED}(>6$ visits per year). Results: Of 127 charts reviewed, 59 were included. Residents primarily treated $19(32 \%)$ patients, and consultants treated $40(68 \%)$ patients. On an average day, $27 \%$ of total discharged patients are treated by a resident and $73 \%$ by a consultant. There was no significant difference in the rate of unscheduled return visits. Consultant notes were documented on $58 \%$ of the resident charts. Regarding the disposition of the unscheduled return visit between those treated by a resident versus a consultant, 8 versus 11 were discharged home with no change in treatment; 3 versus 6 were admitted; 6 versus 13 were discharged home with changes (antibiotics or analgesia changed or added); and 1 versus 10 were referred to an outpatient clinic. There were no significant differences in the return disposition groups or in the time between the index visit and the unscheduled return visit. Conclusion: Resident independence in the ED is important for developing competence and confidence. These preliminary data suggest that there is no significant difference in the unscheduled return visits between patients treated by residents and consultants, suggesting that current supervision respects patient safety.

P85 Effectiveness of a passive educational strategy aimed at improving disinfection of personal items among health care professionals in the emergency department of a tertiary care centre R. Blackman, Medical Student, S. Materniak, BA, S. El Bailey, MD, P.R. Atkinson, MD, FCEM, J. Fraser, Bachelor of Nursing, S. Hull, MD, D. Webster, MD; Dalhousie Medicine, New Brunswick

Keywords: education, emergency medicine, infection control Introduction: Previous studies have suggested that inanimate objects belonging to health care workers may contribute to the transmission of microorganisms from patient to patient in the health care setting. This study examines the effectiveness of posters in the emergency room as a reminder to disinfect personal items. Methods: A group of 10 health care professionals in the emergency department (ED) had selected personal items (cell phone, stethoscopes, pagers, identification cards, sleeves) swabbed to identify microorganisms. Anonymized results were posted in several locations throughout the department, which included a reminder to disinfect personal items. A week after the results were posted, a separate group of 10 health care professionals had the same items tested. The results were analyzed based on type and colony counts of bacteria using a mixed analysis of variance. Results: Fifteen participants (seven pre and eight post) were analyzed based on the cell phone, identification card, and sleeve findings. Overall, a statistically significant increase in colony count was observed in the posteducation period $(p=0.01)$. Mean colony count increased from 38.0 CFU (95\% CI 26.7, 49.3) pre-education to $58.2 \mathrm{CFU}$ (95\% CI 47.6 ,
68.8) posteducation. There was no significant change in the mean number of types of bacteria observed in the pre- and posteducation periods (pre mean 3.9/post mean 3.7, $p=0.7$ ). The bacteria isolated were predominantly gram negative; however, a number of different types of gram-positive bacteria were present as well. Conclusion: Despite its predominant use in health care, the current evidence suggests that the use of passive education alone is insufficient to result in significant change in cleaning practice among health care workers. While our sample size requires the results to be interpreted cautiously, they are consistent with prior studies regarding passive educational strategies. We suggest that health care institutions carefully examine their policies to reduce equipment reservoir sources and look further at this hypothesis.

P86 Community uptake of PulsePoint: using smartphones to crowdsource basic life support for victims of out-of-hospital cardiac arrest

S.C. Brooks, MD, MHSc; Queen's University

Keywords: cardiac arrest, emergency medicine, smartphones

Introduction: PulsePoint is a nonproprietary smartphone application that links willing bystanders with the 911 response to cardiac arrest. The application aims to facilitate immediate bystander basic life support while EMS personnel are en route. After community members download the free software to their smartphones, they receive automated alerts directing them to public location out-of-hospital cardiac arrest emergencies within 300 to 500 metres of their location. The application also identifies nearby public AED locations. Our objective was to quantify the uptake of the PulsePoint application in several US communities. Methods: The PulsePoint application was first launched in San Ramon, California, in December 2011 and has now been implemented in nine additional agencies across California, Kentucky, and South Dakota. Promotional efforts in all communities included targeted marketing to health care provider groups, mass media campaigns, public service announcements in theatres, and the use of social media (e.g., Twitter and Facebook). Data from iTunes, Android Market, and the PulsePoint enterprise server were collected to document the uptake of the application. Results: As of December 31, 2012, there were 41,885 PulsePoint application downloads. The total number of devices registered with active status on the PulsePoint server was 25,209 , accounting for users who removed the application from their device after installation. Of those devices registered, $32 \%$ $(8,067 / 11,167)$ had settings configured to receive cardiac arrest alerts. Conclusion: There has been significant uptake of the PulsePoint application in the United States with an increasing number of users over time. Implementation is under way in Canada. Future work should focus on how to maximize the number of users configuring the cardiac arrest alerts feature to be active, how to encourage retention of the application, and determining the effectiveness of the application with respect to increasing bystander CPR, AED use, and survival for out-ofhospital cardiac arrest victims in the community.

P87 Bed assignment and bed uptake: the bottlenecks in hospital admissions from the emergency department

A. Guttman, MD, V. Verter, PhD, M. Afilalo, MD, X. Xue, MSc, N. Soucy, PhD, A. Colacone, BSc, CCRA; Emergency Department, Jewish General Hospital, McGill University

Keywords: admission bottlenecks, bed access, emergency medicine, time delays

Introduction: Emergency department (ED) boarding is reflected by the inability to get admitted patients out of the ED. Studying patient flow processes is essential for identifying delays aimed at improving hospital bed access for the ED. This study describes the time delays in 
the admission processes of ED patients admitted to short-stay (SS) versus long-stay (LS) medicine units. Methods: ED patients admitted to SS (32 beds) and LS (73 beds) units from Sept. to Nov. 2012 were included. The patient flow process was mapped from the ED admission request to ward arrival. While the hospital discharge process was mapped from discharge orders to bed ready for a new patient. Key data points were collected from the ED database, direct observation, and housekeeping records. Results are expressed as mean and 95\% confidence intervals (CI). Results: Among ED patients ( $n=689$; mean age 70.6 years [ +18.3$] ; 51 \%$ female) admitted to the medicine units ( 375 in SS and 314 in LS), admission request to ward arrival was significantly longer to the LS unit (LS $=26.7$ hours [CI: 24.1-29.3] v. SS $=15.4$ hours [CI: 13.6-17.3]). The majority of these delays were due to the waiting from admission request to bed assignment ( $\mathrm{LS}=24.2$ hours [CI: 21.6-26.8]; SS = 12.8 hours [CI: 11.0-14.6]). There were no significant difference among the two medical units concerning delays from bed assignment to ward arrival (SS $=2.7$ hours [CI: 2.4-2.9]; LS $=2.4$ hours [CI: $2.1-2.7]$ ) and from discharge order to ward departure ( $\mathrm{SS}=1.3$ hours [CI: $1.1-1.6] ; \mathrm{LS}=1.1$ hours [CI: $0.7-1.4]$ ). Delays were similar for preparing the bed for the next incoming ED admission, specifically from inpatient departure to bed ready ( $\mathrm{SS}=1.3$ hours [CI: $1.1-1.5]$; $\mathrm{LS}=1.1$ hours [CI: 0.9-1.3]); from housekeeping notified to bed ready $(\mathrm{SS}=0.9$ hours $[\mathrm{CI}: 0.8-1.1]$; $\mathrm{LS}=0.6$ hours $[\mathrm{CI}$ : $0.5-0.8]$ ); and from bed ready to ED patient arrival (bed uptake) ( $\mathrm{SS}=$ 5.1 hours [CI: 4.3-6.0]; LS = 5.6 hours [CI: 4.4-6.7]). Conclusion: Delayed access to hospital beds, particularly bed assignment and bed uptake, continues to plague ED outflow. Efforts focusing on these two issues hold promise in stemming ED boarding.

P88 Utilizing national emergency department quality indicators to evaluate sepsis care in an Ontario Local Health Integrated Network

W. Supapol, PhD, M. Ackerman, MD, A. Worster, MD; McMaster University

Keywords: emergency department, emergency medicine, quality indicator, sepsis

Introduction: We applied 1 of ICES consensus list of 48 evidencebased quality indicators for Canadian emergency departments (EDs) to assess and compare the quality of sepsis care at 12 Ontario EDs within a single Local Health Integrated Network (LHIN). Methods: We reviewed 100 randomly selected charts with a diagnosis coding of sepsis at each ED for adult visits occurring between July 2009 and June 2011 for serum lactate monitoring. In hospitals with $<100$ sepsis visits over the study period, all visits were selected, including two hospitals with 20 and 6 sepsis visits. Patients discharged home from the ED were excluded. Our two trained data abstractors used standardized data collection procedures and forms and examined all ED records, including laboratory test results, for evidence of lactate testing. Data were simultaneously double abstracted and differences resolved by consensus. We determined the proportion of sepsis visits where serum lactate was tested and $95 \%$ confidence intervals $(95 \%$ CI) and tested whether the observed proportion was significantly different from a hypothetical standard of $90 \%$ using the binomial proportion test. Results: All 12 hospitals agreed to participate, and we reviewed 977 ED sepsis visit charts. Lactate measures were easy to find in laboratory reports at most hospitals. The prevalence of lactate testing for ultimately admitted sepsis patients varied from $0 \%(95 \%$ CI: 0-14) to $93 \%$ (79-97) among the 12 hospitals. Four hospitals had high levels (79-93\%), four had intermediate levels (40-54\%), and four had low levels of serum lactate testing $(0-17 \%)$. Ten hospitals were significantly different from the standard of $90 \%$. Conclusion: There is a large variation in the prevalence of lactate testing among hospitals in the LHIN. Lactate measures are easy to find in charts and are a useful indicator for quality assurance initiatives.

\section{P89 Prevalence of drug use in injured British Columbia drivers}

J.R. Brubacher, MD, H. Chan, PhD, M. Asbridge, PhD, W. Martz, $\mathrm{PhD}$, S. Macdonald, PhD, S. William, MD, R. Purssell, MD, A. Lund, MD, J. Eppler, MD; University of British Columbia

Keywords: drug driving, emergency medicine, motor vehicle crashes Introduction: Impaired driving is a major public health problem, but drug-impaired driving remains poorly understood. Canadian roadside surveys and coroner's data suggest that drug driving may be as prevalent as drunk driving. A study from the 1980s found that drug use was common in injured drivers treated in a Toronto trauma centre, but the prevalence of drug use by injured Canadian drivers has not been studied recently. This study is part of an ongoing project investigating the role of cannabis in causing motor vehicle crashes. The objective is to determine the prevalence of drug use among a sample of injured drivers treated in British Columbia trauma centres. Methods: This study was approved by our institutional research ethics board. Drivers treated in the emergency departments (ED) at three participating BC trauma centres (2010-2012) were identified prospectively. Excess blood remaining after clinical use was obtained and analyzed at the $\mathrm{BC}$ provincial toxicology laboratory to determine the presence and concentration of cannabis, alcohol, and other drugs that could impair driving ability. Results: To date, 783 drivers met the study criteria and had blood analyzed. The average age of these drivers was 42.8 years (median $=44, \mathrm{IQR}=26-55) ; 63.9 \%$ were male. Alcohol was present in $19.4 \%$ of drivers (among these $83.6 \%$ had blood alcohol concentration $>0.08 \%$ ), and $11 \%$ tested positive for cannabis (THC and/or metabolites). Other commonly detected recreational drugs included cocaine and metabolites $(3.7 \%$ and $7.2 \%)$ and amphetamines $(1.8 \%)$. Medications including benzodiazepines $(4.2 \%)$ and diphenhydramine $(12.4 \%)$ were also detected in these drivers. Conclusion: Cannabis and other drugs are commonly detected in injured BC drivers. Further research is needed to determine whether drivers who use drugs are more likely to cause crashes.

\section{P90 Practice patterns of graduates of the McMaster University CCFP(EM) residency program}

J.W. Matte, BSc, MD, D. Lin, MD, CCFP(EM); McMaster University

Keywords: emergency medicine, medical education, practice patterns Introduction: The CCFP(EM) residency program was originally intended to improve emergency care delivered by family physicians, not to produce career emergency physicians. However, studies have shown that the majority of $\operatorname{CCFP}(\mathrm{EM})$ graduates practice full-time emergency medicine and work strictly in urban/suburban hospitals as opposed to rural centres. Study objectives are to determine the practice setting of graduates of the McMaster University Family Medicine/Emergency Medicine residency program that leads to a Certification of Special Competence in Emergency Medicine (CCFP[EM]). Methods: Qualitative assessment of graduates of McMaster University CCFP(EM) program from 1982 to 2012 via a Web-based, email-distributed survey. An introductory email will be sent to participants prior to survey completion. An information and consent sheet will be included with the survey, and completion implied consent. Surveys were distributed using modified Dillman methodology. Data will be analyzed with simple statistics, and subgroup comparison will be conducted with chi-square and $t$-test analysis. Results: This study will determine the current practice type and location of graduates of the McMaster CCFP(EM) program and compare it to similar programs in Ontario. Secondary outcomes include association 
between practice type and location with demographic factors, prior medical training, prior practice intentions, and current job satisfaction scores. Conclusion: This study will attempt to demonstrate whether a higher percentage of McMaster CCFP(EM) graduates have a blended family/emergency medicine practice, practice in rural areas, and have higher job satisfaction. This is in the hope that other programs may consider adopting designated rural EM streams similar to McMaster University.

P91 The effect of medical trainees on pediatric emergency department flow: a discrete event simulation modeling

E.D. Genuis, MD, Q. Doan, MDCM, MHSc, $\operatorname{PhD}(\mathrm{C})$ FRCPC; University of British Columbia

Keywords: discrete event simulation, emergency medicine, learners, overcrowding

Introduction: Providing patient care and medical education are both important missions of teaching hospital emergency departments (EDs). With medical school enrolment rising and ED crowding becoming an increasingly prevalent issue, it is important for EDs to find a balance between these two potentially competing goals. Our objective is to investigate how the number of trainees in a pediatric ED (PED) affects patient flow. Methods: A DES model of an urban tertiary care PED was created and validated. Thirteen different PED trainee schedules, which ranged from averaging zero to six trainees per shift, were input into the DES model and outcome measures were determined using the averaged output of five model iterations. The outcome measures investigated were wait time (WT), total ED length of stay (LOS), and rates of patients leaving without being seen (LWBS) for PED patients overall and stratified by Pediatric Canadian Triage and Acuity Scale (CTAS) acuity level. Results: Average LOS rose by 7.3 minutes $\left(r^{2}\right.$ 0.99 ) for every additional trainee per shift working in the PED. The relationship between the number of trainees and WT varied with patients' acuity level and with the degree of PED utilization. Patient WT decreased as the number of trainees increased, primarily for lowacuity visits and when the PED was not operating at full capacity. With rising numbers of trainees, the LWBS rate decreased in the whole department and in the CTAS 4 and 5 patient groups, but it rose in patients triaged as CTAS 3 or higher. Conclusion: The results of this study demonstrate that PED trainees have an impact mainly on patient LOS and that the impact on WT differs between patients presenting with varying degrees of acuity. Findings will assist PEDs in finding a balance between providing medical education and timely patient care.

P92 Fostering leadership in emergency medicine education: enhancing resident learning through curriculum redesign

A. Selvig, MD, S. Smith, MD, J. Zimmer, PhD; University of Saskatchewan, Department of Academic Family Medicine

Keywords: administration, curriculum, education, emergency medicine

Introduction: The University of Saskatchewan Family Medicine Emergency Medicine (FM EM) residency program has developed and implemented a unique 4-week administrative rotation run through two satellite teaching sites in the Regina Qu'Appelle Health Region. Faculty identified common administrative challenges facing emergency department (ED) physicians and hoped to enhance EM education by providing additional learning opportunities on department flow, teaching of medical learners, business management and finance, EMS/police collaboration, and medicolegal issues. Methods: Our study gathered feedback to better understand the perceived value the rotation serves as a component of the FM EM training program at the University of Saskatchewan. Data were collected through interviews and surveys of the past residents who have completed the rotation. Results: The administrative rotation was introduced in 2009, and 17 residents have completed the rotation. All 14 residents who agreed to participate in our study deemed the administrative rotation valuable to their EM education. The rotation components that most impacted current practice as they transitioned into staff physicians were shifts and sessions that offered strategies to manage department flow and teaching medical learners. The discussions on physician finance and medicolegal challenges were also considered to be very important. Residents found the collaboration shifts had less impact on EM practice. Furthermore, the majority of residents suggested that the timing of the rotation within the training year affected the overall value of the rotation. Conclusion: Past residents found the administrative rotation addressed important challenges faced by ED physicians as they transitioned from residents to attending physicians. The residents generally feel that this rotation added significant value to their training and has positively impacted their current practice. As guided by the feedback of past residents, we feel it is important to continue to offer this administrative rotation as a component of the FM EM residency curriculum at the University of Saskatchewan.

P93 Online social media use in the emergency department: a national survey of emergency physicians and trainees

M. Ben-Yakov, MDCM, A. Kayssi, MD, MSC, J. Chu, MD, MSC, FRCP, C. Hicks, MD, MED, FRCPC; University of Toronto

Keywords: emergency medicine, ethics, privacy, social media Introduction: The use of online social media websites (OSM) by health care providers to retrieve medical information on patients raises many ethical dilemmas. Although professional and legal bodies have cautioned physicians regarding the use of OSM, the frequency with which emergency physicians (EPs) and trainees (residents and medical students) look up their patients on Facebook or Google during their ED visit is currently unknown. Methods: We validated and administered an online survey study of Canadian EPs and trainees to explore the current use of Facebook and Google to search for patients presenting to the emergency department. Participants were contacted using the Canadian Association of Emergency Physicians (CAEP) emailing list. Physicians, residents, and medical students at our institution were also invited to participate by email. Results: We received 530 responses (response rate $47.3 \% ; 34.9 \%$ medical students, $15.5 \%$ residents, and $49.6 \%$ staff). The majority of respondents had an active Facebook account $(74 \%)$ and had used an electronic medical record system in the ED over the past year (75.9\%). Sixty-four participants (13.5\%) had used Google to research a patient and $10(2.1 \%)$ had looked up patients on Facebook. One-third of physicians considered Facebook use to learn about a patient as "very unethical," with violation of patient confidentiality and dignity most frequently cited as a concern. A respondent's views on the ethics of using OSM in the ED was the only significant predictor for whether a respondent used OSM to look up a patient. Conclusion: This is the first survey of OSM use in the ED. We found no age or training-level differences in attitudes toward OSM use in the ED among our respondents. Ethical and professional concerns regarding the use of such modalities in the ED highlight the need for clear policies to help guide physician conduct.

\section{P94 Remote death certification in Québec avoids ambulance transports: first 2 years in operation}

A.B. Tanguay, MD, MSc, D. Hébert, MSc, G. Foldes-Busque, PhD, R. Fleet, $\mathrm{MD}, \mathrm{PhD}$; CSSS Alphonse-Desjardins

Keywords: death certification, emergency medicine, prehospital Introduction: The Unité de coordination clinique des services préhospitaliers d'urgence (UCCSPU) is a clinical entity modeled after 
the concept of base hospital, created to improve access and quality care for patient users of the prehospital emergency medical system (EMS). In Québec and in most other provinces, deceased persons are usually transported by ambulance to a hospital for death certification by a physician. Certifying a death remotely could optimize the EMS and emergency departments (ED). Methods: The UCCSPU implemented the remote death certification project approved by the Québec Ministry of Health and Social Services, the Coroner's Office, and the Québec College of Physicians. The emergency medical technician (EMT) applies the protocol and provides data to the nurse at the UCCSPU. The physician at the UCCSPU can remotely prepare the death certificate. The ambulance team becomes available for other calls. The UCCSPU contacts the funeral home or the coroner on duty. The study collected retrospective data from all remote death certification requests from EMTs over a 21-month period. The data were abstracted from the administrative database of the UCCSPU by the nurse coordinator and verified by a physician supervisor. Results: From April 11, 2011, to December 31, 2012, the UCCSPU responded to 611 remote death certification requests from EMTs. In all, 529 $(86.6 \%)$ death certificates were prepared remotely. Of those 529 deaths, 356 deaths were from natural causes, and 176 deaths were coroner's cases. Conclusion: Implementing this project was successful in avoiding more than 500 transports to the ED. Further costanalyses studies are planned.

P95 Potential value and utility of technology to foster interprofessional discussion and education among emergency medicine health care professionals: a preliminary needs assessment

J. Riley, MD, L. Barratt, BScN, L. Rozmovits, PhD; St. Michael's Hospital

Keywords: emergency medicine, IPE, qualitative, technology Introduction: The emergency department (ED) is a complex environment with competing priorities and ever-changing information where health professionals (HP) have little to no time during a shift to pause, discuss, and reflect on clinical experiences, policies, or new research. The nature of shift work and loss of personal time to attend formal interprofessional education (IPE) and meetings pose challenges, and online resources may have the potential to create a shared, inclusive, conveniently accessed platform for dialogue and education. The objective was to explore ED stakeholders' perspectives of current culture of IPE and value with new technology. Methods: Twelve semistructured interviews exploring new online technologies were conducted within a Level 1 trauma, academic, inner-city ED (six nurses, five physicians, one social worker). The method of constant comparison was used for analyses and included searches for disconfirming evidence. Results: Webcasts offered flexible access on shift or at home but were seen as one-directional, unengaging, and unlikely to be used. Interactive discussion forums would enable dialogue for evolving practice and new research and were preferable to webcasts, but concerns about confidentiality, moderating, and uptake by the collective would need to be addressed to ensure sustainability. An online ED centralized repository for information was considered appealing and had potential to evolve into an interactive resource. Barriers to new technology were an unwillingness to "work" off shift and the loss of face-to-face interaction. Personal engagement to discuss sensitive topics in an unhurried, nonstressful environment during IPE formal meetings and rounds was preferred. Conclusion: Introducing online technologies in the ED to support IPE and discussion should be viewed with caution. New opportunities must fill a clearly defined need, be value added, and enhance clinical practice through consolidating and simplifying existing resources. Creating a collaborative website to improve process and function as a knowledge repository may lead to a future interactive resource for shared learning across professions.
P96 Assessing equity of care in an emergency department using patient experience surveys

H.H. Chiu, MHA, N. Batara, MHA, R. Stenstrom, MD, PhD, C. Jones, RN, MEd, L. Cuthbertson, BHScOT, MEd, E. Grafstein, MD, L. Carley, MA; St. Paul's Hospital

Keywords: emergency medicine, equity, patient experience, quality of care

Introduction: Measuring equity of care is difficult in emergency departments. Our objective was to assess equity in an ED as measured by patient experience surveys administered via different methods. Methods: A prospective cohort study involved a convenience sample of validated patient experience surveys from marginalized (equity) populations (elderly, low income, homeless, and mental health or substance use issues [MH/SA]) collected over an 11-week period at an urban inner-city ED. The baseline control population included all patients who responded to a regular mailed survey from the same ED. We compared response rates between equity patients pseudorandomized to receive face-to-face interviews versus handout and the baseline control. ED staff were blinded to the study objectives. Results: Compared to the equity visits, elderly patients were overrepresented ( $p=$ 0.031 ), while the low-income, homeless, and $\mathrm{MH} / \mathrm{SA}$ subgroups were underrepresented among the mailed survey respondents $(p<0.001)$. There were 101 equity patients who completed the patient experience surveys compared to 310 respondents for the mailed survey. Surveys administered by interview or handout had a substantially higher response rate than surveys administered by mail. Equity patients were more likely to have been admitted, to have taken an ambulance to the $\operatorname{ED}(p<0.001)$, and to have a CTAS level of 1 to $3(p=0.041)$. There was no statistical difference in time to MD or ED LOS between groups. All 68 face-to-face interviews were completed versus 33 of 43 handout surveys. Seven of the nine survey questions showed no difference in positive response rate, one showed increased satisfaction, and one showed decreased satisfaction in the equity group. Conclusion: Patient experience surveys provide valuable information about equity of care from the patient perspective. Our findings support routine stratification of survey results by relevant sociodemographic characteristics to monitor equity of care in EDs.

P97 Physicians' perspectives on effectiveness of intranasal midazolam for emergency department procedural sedation in children L. Colpitts, MD, CCFP; McMaster University

Keywords: emergency department, emergency medicine, intranasal midazolam, procedural sedation

Introduction: Children regularly present to the emergency department requiring treatment facilitated by procedural sedation. Intranasal midazolam is a favourable medication for several reasons: rapid onset, ease of use, amnestic and anxiolytic qualities, and limited duration of action (1-3). Its utility is well documented in dental and radiologic procedures (4-8); however, the evidence for its use in the emergency department remains sparse. We aim to evaluate the effectiveness of intranasal midazolam for procedural sedation in pediatric patients in the emergency department. Methods: This is a prospective case series. Following the use of intranasal midazolam for procedural sedation, emergency physicians will complete a survey regarding the effectiveness of the medication. Key questions include the sedation score achieved, need for additional doses or adjuvant medications, and success rate of the undertaken procedure. The pediatric patients identified will be at the discretion of the physician in terms of age, necessary procedure, and the need for procedural sedation. Results: Analysis will include quantitative responses consisting of the mean physician satisfaction (scale of 1 to 5), the mean dose used $(\mathrm{mg} / \mathrm{kg})$, the mean number of doses, the mean sedation score achieved, and the proportion of com- 
pletion of undertaken procedures. Qualitative information will include the types of procedures performed and the need for adjuvant medication. Conclusion: We anticipate that physicians use intranasal midazolam for a range of procedures requiring sedation in the emergency department. This study will provide information on the utility of this method of sedation and its effectiveness in the emergency department for pediatric patients.

P98 Personal digital assistant use by emergency medicine residents in Ethiopia: an educational pilot on behalf of the TAAAC(EM)

\section{collaboration}

M. Ben-Yakov, MDCM, C. Hunchak, MD, CCFP(EM), M. Landes, MDCM, CCFP(EM), MPH; University of Toronto

Keywords: decision making, education, emergency medicine, mobile technology

Introduction: Personal digital assistants (PDAs) have revolutionized medical education and clinical practice in emergency medicine (EM) in the developed world and have been shown to improve clinical decision making, reduce medical error, verify harmful drug interactions, and enhance medical education. Technological change happens rapidly in the developed world, leading to a surplus of functional, discarded tools that might be repurposed for use in other settings. In Ethiopia, where the Toronto Addis Ababa Academic Collaboration in Emergency Medicine (TAAAC-EM) helps train postgraduate EM residents at Addis Ababa University (AAU), PDAs are not in use due to high costs, unreliable Internet access, and poor availability. Residents lack consistent access to EM resources while on duty in the ED. To address this gap in resident education and patient care, we piloted $\mathrm{Palm}{ }^{\circledR}$ TX $^{\text {TM }}$ PDA use among second-year Ethiopian EM residents to assess the feasibility of incorporating PDAs into the AAU EM residency program. Methods: We distributed Palm TX PDAs containing five standardized offline applications relevant to EM practice (General EM, Peds EM, drug and laboratory databases, and an ACLS/PALS application) to five senior residents in February 2012. Residents were trained in PDA use by video; email and in-person tech support was available. A 20-item semistructured questionnaire was developed and distributed to the residents to complete at 1 month after initial experience with the PDA. We inquired about prior technology experience, the ease of use, utility, contextual applicability, and perceived barriers to PDA use Results: All senior residents completed the questionnaire $(n=5)$. All felt comfortable with computer use in general. PDAs were used regularly both on and off duty in the ED and were perceived to help with most aspects of patient care. PDAs were of particular value in referencing the bedside management of patients presenting with infrequently encountered conditions. No major technical difficulties were reported, although the numbers are too small to draw specific estimates from. Conclusion: PDAs were perceived by senior Ethiopian EM residents to be a useful educational adjunct without major obstacles to implementation. We plan to offer standardized PDA access to all AAU EM residents followed by a more formal assessment of their utility in this low-resource environment.

P99 The functional status assessment of seniors in the emergency department (FSAS-ED): its clinical utility

N. Veillette, PhD, M. Beaudoin, BSc, M. Sirois, PhD, M. Stern, BSc, L. Leclair, PhD (Cand.); Université du Québec à Trois-Rivières

Keywords: assessment tool, emergency medicine, functional assessment, geriatrics

Introduction: Funded by a CIHR grant, the Functional Status Assessment of Seniors in the Emergency Department (FSAS-ED) was devel- oped to assess older adults visiting the ED. Objectives for the presentation are to provide information on the purpose of the FSAS-ED and to describe a study to establish its clinical utility. Methods: First, an implementation pilot study for the FSAS-ED was carried out with a convenience sample of 24 patients to ensure the applicability of the tool. Subjects were medically stable and screened by ED staff as to having limitations in activities of daily living. Then, based on medical chart review, a longitudinal case-control study was conducted with 48 controls randomly selected and matched to subjects on specific criteria (age, gender, reasons for ED consultation, time of consultation). At discharge from the ED, comparisons were made regarding inpatient admission, transfer to another facility, or return home rates. Additional comparisons were conducted after 3 and 6 months following the initial $\mathrm{ED}$ visit regarding return to the $\mathrm{ED}$, hospitalization, transfer to longterm care, or death rates. Results: Subjects evaluated using the FSASED were mostly female (79\%) with an average age of 84.3 years. Their return home rate was $79 \%$ compared to $44 \%$ for controls. After 3 months, when compared to controls, fewer subjects returned to ED or were hospitalized and none were placed in care or died. After 6 months, subjects still did better than controls, with no deaths, and $4 \%$ were placed in long-term care, compared to a quarter of control patients deceased and $8.5 \%$ placed in long-term care. Conclusion: Results suggest that using the FSAS-ED may reduce some adverse outcomes in terms of return to ED, hospitalization, being placed in longterm care, and death.

P100 Why university athletes do not seek medical attention following concussion symptoms

C. Lamfookon, MD, J. Delaney, MD, G. Bloom, PhD; McGill University

Keywords: concussion, emergency medicine, underreporting

Introduction: Concussions are a common occurrence in sport. Athletes who continue to play while still symptomatic from a concussion are believed to be at risk for more severe injury, and repeated concussions may also result in progressive and cumulative neurologic and neuropsychological impairment. We endeavoured to learn more about athletes' understanding of concussions and, in particular, the reasons they may not seek medical attention while symptomatic from what they believe to be a concussion. Methods: We enrolled athletes participating in varsity football, soccer, basketball, ice hockey, and rugby from two universities. The athletes completed questionnaires focused on collecting individual demographics as well as identification and underreporting of concussion symptoms in the past 12 months. They were asked a series of questions pertaining to why they did not seek medical attention. Results: Preliminary results of the 470 participants enrolled indicate that athletes who did not report their concussive symptoms did so most frequently so as not to be removed from the game, due to fear of disappointing the team, and because they did not feel their symptoms were significant. Interestingly, some of the participants stated that they did not disclose their concussive symptoms due to a perceived lack of access to medical personnel. Conclusion: No definitive conclusions can be made at this time as the data continue to be analyzed; however, this study suggests that education about the risks of continuing to play while symptomatic from a concussion and improving access to medical personnel have the potential to decrease underreporting of concussion symptoms by athletes.

P101 In situ simulation in an emergency department: a comparison of evaluation tools

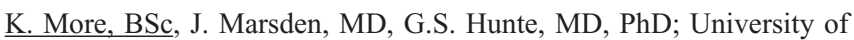
British Columbia

Keywords: emergency medicine, evaluation, in situ simulation 
Introduction: Emergency department performance depends on crossdisciplinary coordination, communication, and collective learning. In situ simulation is a research and education strategy that brings deliberate practice and learning of teaming skills into the workplace to improve nontechnical skill performance in dynamic ad hoc teams. We sought to select a validated teamwork assessment tool to evaluate team performance during in situ simulation in an emergency department. Methods: A scoping review of the health and organizational literature was conducted to find existing teamwork assessment tools. Three reviewers compared and contrasted the tools according to published guidelines for designing team performance measurement in health care-based simulation training. Hand searching and contact with authors were not undertaken. Results: The review returned seven relevant assessment tools: the Anesthetist Nontechnical Skills tool, the Emergency Department Nontechnical Skills assessment tool, the Clinical Teamwork Scale, the Communication and Teamwork Skills assessment tool, the Ottawa Global Rating Scale, the Mayo High Performance Teamwork Scale, and the TeamSTEPPS observation tool. Although no published tool met all criteria, the Clinical Teamwork Scale (CTS), a theoretically and empirically grounded and psychometrically valid generic team assessment tool developed to measure key clinical teamwork skills during simulation exercises and in everyday clinical care, met most criteria and has recently been piloted to assess team performance in in situ trauma simulations. Conclusion: The goal of this review was to determine the current scope of the literature regarding evaluation of team performance in health care. The available tools differ with respect to unit of analysis (individual v. team), target specialty (specific v. generic), type of behaviours evaluated, description of behaviours, scoring criteria, and strength of psychometric evaluation. The CTS offers a brief, valid, and reliable assessment tool for specific, observable actions that can be evaluated by trained observers and combined with reflexive self-assessment.

P102 A systematic review of ethanol and fomepizole use in pediatric toxic alcohol ingestions

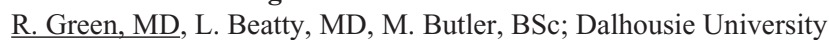

Keywords: emergency medicine, ethanol, fomepizole

Introduction: The optimal antidote for the treatment of ethylene glycol or methanol intoxication in the pediatric population is not known. The object of this systematic review is to describe the available data on the use of ethanol and fomepizole for methanol and ethylene glycol intoxication in children. Methods: A broad and systematic search of MEDLINE and EMBASE was conducted. Published studies involving the use of ethanol or fomepizole, or both, in pediatric patients $(<18$ years old) who presented within 72 hours of toxic alcohol ingestion were included. Our search yielded a total of 25 case series for analysis after identification by 2 independent authors. No randomized controlled trials and no head-to-head trials were identified. Variables were evaluated for all publications using a standardized data collection form. Results: In the available literature, 74 patients with toxic alcohol ingestion were identified. Sixty-two $(83.8 \%)$ were treated with ethanol (31 EG, $31 \mathrm{Me}), 7$ (9.5\%) with fomepizole (1 Me, $6 \mathrm{EG})$, and 5 (6.8\%) with both antidotes ( $1 \mathrm{Me}, 4 \mathrm{EG})$. Mortality outcomes were reported in 57 cases, with one death reported in a patient who received ethanol $(1 / 24 ; 4.2 \%)$. Hypoglycemia was reported in 2 patients (ethanol 1; fomepizole 1). Other adverse events were uncommonly reported. Conclusion: The data supporting the use of one antidote are inconclusive, based on reported mortality and adverse event profiles. Further investigation is warranted.

P103 Qualitative analysis of teaching satisfaction among residents rotating through three emergency departments
M. Bhimani, MSc, MD, CCFP(EM), D. Grushka, MD CCFP(EM); University of Western Ontario

Keywords: education, emergency medicine, residents emergency department, teaching emergency department

Introduction: The emergency department (ED) provides unique opportunity for learning. The undifferentiated ED patient population harbours illness specific to many disciplines, provides opportunity for applying basic diagnostic skills, and enables inculcation of resuscitation skills. Studying the quality of teaching provided allows improvement in the skills of ED clinician-teachers. We sought to conduct a study examining the teaching experience of residents within three EDs. Methods: Residents rotating through two tertiary care academic EDs and one community ED were surveyed using an online link. The residents were asked to provide their training level, the site, and shifts worked and surveyed on the teaching provided. Responses were collected on the survey-monkey tool and subject to qualitative analysis using the online data analyzer. Results: A total of 340 responses were analyzed. Most residents (94.8\%) worked in the urban EDs; $82.3 \%$ of residents felt they received bedside teaching, but this was more positively reflected among urban (82.9\%) versus rural (68.8\%) residents, and $38.5 \%$ of residents received procedural training, but this was to a lesser degree among community trainees (28.6\%). Only $26.8 \%$ of overall residents participated in a resuscitative case but less so in the community $(14.3 \%)$. While $63.2 \%$ of collective residents felt they needed more bedside teaching, this was more so among community residents $(85.7 \%)$, who also felt they needed more procedural training $(85.7 \%)$ compared to their urban colleagues $(55.7 \%)$. Conclusion: Our data demonstrate that while teaching overall was rated highly, communitybased residents reported getting less training in procedural skills and resuscitation. However, the exposure to procedural skills and resuscitation is low among all residents. Also, bedside teaching was provided less frequently in the community. Community EDs should provide more bedside teaching, while all EDs should involve residents more frequently in procedures and resuscitation.

P104 Common diagnoses and outcomes of elderly patients presenting to the emergency department with nonspecific complaints

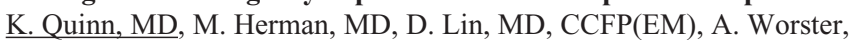
MD; McMaster University

Keywords: elderly, emergency department, emergency medicine, presenting complaint

Introduction: Patients often present to the ED with nonspecific complaints such as generalized weakness or feeling unwell. Previous studies indicate that elderly patients with these complaints are at greater risk for life-threatening illnesses than younger patients or those with specific complaints. We sought to evaluate the final diagnoses and outcomes of elderly patients presenting with nonspecific complaints. Methods: Two trained data abstractors used standardized data collection procedures and forms to independently review all records of patients over age 70 years presenting to two academic EDs in 2010 with nonspecific complaints as defined by the Canadian Emergency Department Information System (CEDIS): fatigue, general weakness, and feeling unwell. Outcomes of interest were ED discharge diagnosis, admission to hospital, length of stay in hospital, and ED revisit if discharged. Differences between abstractions were resolved by consensus. Results: Of the 743 patients included in the study, 265 were excluded because they had dizziness, vertigo, or a specific complaint. A total of 419 patients $(87.7 \%)$ presented with weakness, and 59 patients $(12.3 \%)$ presented with general fatigue or felt generally unwell. The most common diagnoses for these patients were UTI (7.3\%), TIA (6.5\%), and dehydration (3.6\%). In total, there were 11 admissions with a median length of stay 
of 5 days. Four patients underwent surgery and four patients died in hospital. Eighty-one patients $(16.9 \%)$ had a repeat ED visit within 30 days of ED discharge. Conclusion: Contrary to previous studies, our results do not show that elderly patients presenting to the ED with nonspecific complaints are necessarily at higher risk for life-threatening illnesses. The most common diagnoses are UTI, TIA, and dehydration. Most patients can be discharged safely, although there is a relatively high rate of ED revisit within 30 days.

\section{P105 Factors in uptake by EMS of a new MedicAlert EnRoute} Notification technology

A. Carter, MD, M. Bohrer, PCP, J.L. Jensen, ACP MAHSR; Emergency Health Services Nova Scotia

Keywords: emergency medicine, EMS, health technology, Theory of Planned Behaviour

Introduction: MedicAlert EnRoute Notification (MAN) allows paramedics to download the full Canadian MedicAlert Foundation (CMAF) record. Electronic medical records and the integration of multiagency data are increasingly important. Understanding barriers and facilitators to the uptake of new technologies will be key to successful implementation. Methods: This qualitative Theory of Planned Behaviour (TPB) study assessed paramedic and medical communications officer (CO) intent to adopt the use of MAN, with three variables: behavioural beliefs (BB - attitude), subjective norms ( $\mathrm{SN}$ - social pressure), and perceived behavioural control (PBC - perceived control to do the behaviour). Telephone interviews were conducted using a structured guide with a planned sample of 12 paramedics and 12 COs and then transcribed and independently coded by two investigators. Differences were resolved by consensus. Results: For the 12 COs, 4 BB codes emerged: CMAF as information source (11/12 made some comment); Importance of timing of MAN use (9/12); Knowledge/awareness of MAN (7/12); and Privacy concerns (7/12). Groups identified under SN: Patients/family (6/12), Management (6/12), Health care providers (6/12), and CMAF (3/12). Three PB codes emerged: Getting the number from caller (12/12); Design/functionality of MAN (10/12); and Time it takes $(9 / 12)$. For the 12 paramedics, five BB codes emerged: CMAF as information source (12/12); Knowledge/Awareness of MAN (10/12); Importance of timing of MAN use (7/12); Accessibility/availability of MAN (7/12); and Functionality of MAN
(6/12). Groups noted under SN: Patients/family (6/12); Management (6/12); Health care providers (4/12); and CMAF (3/12). Under PB, four codes: Tablet interface (12/12); Time it takes (10/12); MAN technology design (7/12); and Bracelet usability (5/12). Conclusion: Perceived behavioural control was most significant in the COs' intention to use this new technology, while it was behavioural beliefs for paramedics. Codes will be further explored and quantified by survey. This will be of value in refining MAN and in understanding barriers and facilitators to the uptake of health technologies.

P106 Stethoscope contamination with methicillin-resistant Staphylococcus aureus in an inner-city emergency department

J. Thom, MD, G. Saito, MD, Y. Wei, BSc, P. Gnana, BSc, B. Willey, MSc, B. Borgundvaag, PhD, MD; University of Toronto

Keywords: emergency medicine, infectious disease, MRSA, stethoscope

Introduction: Due to frequent exposure to individuals colonized and infected with methicillin-resistant Staphylococcus aureus (MRSA), health care workers and their equipment are at increased risk for contamination. The prevalence of stethoscopes contaminated with MRSA has been examined in both inpatient and outpatient settings; however, little is known about the contamination rates within the emergency departments that bridge these two settings. The goal of this investigation was to screen stethoscopes of all health care providers within an inner-city emergency department for MRSA and other opportunistic pathogens. Methods: A convenience sample of stethoscopes was obtained from all consenting emergency physicians and nurses, respiratory therapists, paramedics, as well as from consulting physicians from other departments within a 472-bed tertiary care hospital. All samples were cultured for MRSA, methicillin-susceptible Staphylococcus aureus (MSSA), and other pathogens using standard microbiology techniques. Results: None of the sampled stethoscopes grew MRSA. Ninety (96.7\%) stethoscopes grew mixtures of environmental, skin, and fecal bacteria. Species capable of causing opportunistic infections, such as MSSA, Pseudomonas aeruginosa, and Acinetobacter baumannii, were isolated from seven stethoscopes (7.5\%). Conclusion: Our results suggest that stethoscopes present a potential vector for transmission of MRSA and other opportunistic pathogens to patients in the health care environment. While no MRSA-contaminated stethoscope 